\title{
Performance Characterization of USRPs
}

\author{
Gayathri Ramasubramanian
}

Thesis submitted to the faculty of the Virginia Polytechnic Institute and State University in partial fulfillment of the requirements for the degree of

Master of Science

In

Electrical Engineering

Carl B. Dietrich, Chair

Jeffrey H. Reed, Co-Chair

Yaling Yang

Joseph R. Godfrey

5 September 2014

Blacksburg, VA

Keywords: USRP, Calibration, Characterization

(C) 2014. Gayathri Ramasubramanian. All Rights Reserved. 


\title{
Performance Characterization of USRPs \\ Gayathri Ramasubramanian
}

\begin{abstract}
Software Defined Radios (SDRs) are systems in which components such as filters, modulators, demodulators, etc., typically implemented in hardware are instead implemented in software. Thus, SDR systems shift signal processing from analog to digital domain. The signal processing is performed in reconfigurable devices like General Purpose Processors (GPP) or Field Programmable Gate Arrays (FPGA). The resulting flexibility of SDR systems brings in many advantages to wireless communications such as improved interoperability, adaptation capability and more future-proof hardware.

Various prototype/laboratory friendly hardware are available such as Universal Software Radio Peripherals (USRPs), developed by Ettus Research, which in combination with software interfaces such as GNU Radio can be used to design and implement a Software Defined Radio(SDR) system. This has in turn enabled numerous research opportunities and advancements in field of wireless communication. However one major drawback of these simple USRP devices is that they are un-calibrated in terms of power or voltage and hence give the results in relative terms/counts. This poses a disadvantage in real world scenarios where parameters such as power measured in milliwatts or $\mathrm{dBm}$, are used to design, implement, and test devices and systems and to collect and interpret experimental results. Lack of connectivity with these metrics leads to the USRP devices being confined to proof of concept implementation and demonstrations, and limits their utility for experimentation.

In this thesis an attempt has been made to calibrate the USRPN210 (with WBX daughter board) devices and derive some calibration factors that would help relate the experimental results
\end{abstract}


obtained from the USRP and GNU Radio combination to real world metrics. This also makes the result-set clearer and easily understandable. Several experiments were conducted to understand and characterize the performance of the USRP under different conditions. Experiments such as determination of 1-dB compression point, Third order intercept point helped understand the linearity range of the device under transmitter and receiver operations. The $3^{\text {rd }}$ order input intercept point for USRPN210 device with WBX board with calibration was found to be around $0.25 \mathrm{dBm}$, which is closer to the expected value of $0 \mathrm{dBm}$ at $400 \mathrm{MHz}$. Amplitude and frequency stability tests over the operational frequency range of the daughter board helped to note if change in frequency produced any variation in the received or transmitted power. Also variation with respect to gain helped derive a reference table for transmitted power, which could be used in for future experiments. Conducting these experiments over a sample of 10 USRPs helped in obtaining standardized values and benchmarking them. The factors obtained were also used in some experiments like path loss modeling, position location estimation in order to determine their effectiveness and impact on such applications.

Although, these factors obtained may not be directly applicable to all USRP devices, as in any analog circuitry, and also since they are conducted relative to some specific applications such as the predefined spectrum analyzer utility of GNU Radio (UHD_FFT.grc), they do help to understand the basis of derivation of calibration metrics resulting from the various experiments and also provide a platform for future work in this area to obtain more uniform and globally acceptable results. All photos by the author unless cited, 2014. 


\section{ACKNOWLEDGMENTS}

First and foremost, I would like to thank my advisor Dr. Carl Dietrich whose support and guidance helped pave the way for the successful completion of my Masters. His patience and accommodative nature has been a key ingredient in my progress. Secondly I would like to thank my committee members Dr. Reed, Dr. Yang and Dr. Godfrey whose courses provided valuable knowledge and insights for this thesis work. Such an experiment-oriented work would have been impossible to complete without all the valuable inputs from Randall Nealy from the very start, who always answered my never ending queries with his usual smiling demeanor, and Dr. Vuk Marojevic who provided the laptops and USRP devices without hesitation until all my work was done. I would also like to take this platform to thank Dr. De La Ree, my TA faculty members Dr. Tront and Dr. McPherson who have always mentored and supported me in my work. A warm thank you, to all my friends and colleagues, who made Blacksburg an enjoyable place to stay and study.

I would like to thank my family members who have been a constant source of support and encouragement. My mother for her endless love, my father for having always pushed me to explore my capabilities, Nithya for being a friend more than a sister, my niece Avighna who provided the much needed joyful respite during long lab hours in her own way and my in-laws for all the love and understanding they showed in various occasions. Last but not the least, I would like to thank my husband Arvind who has been my pillar of strength throughout this course of my education. He has been there by my side guiding me in every step of the way right from admission to the defense. Achieving a MS degree would have been left a dream without his constant encouragement, unwavering faith in my ability and most of all his love. I thank him for 
always being there for me. Finally, I would like to dedicate this to my elder sister Deepa who has been an inspiration to me in my life and who now blesses me from her heavenly abode. I would also like to thank the almighty unconditionally for having provided and blessed me with these opportunities. 


\section{TABLE OF CONTENTS}

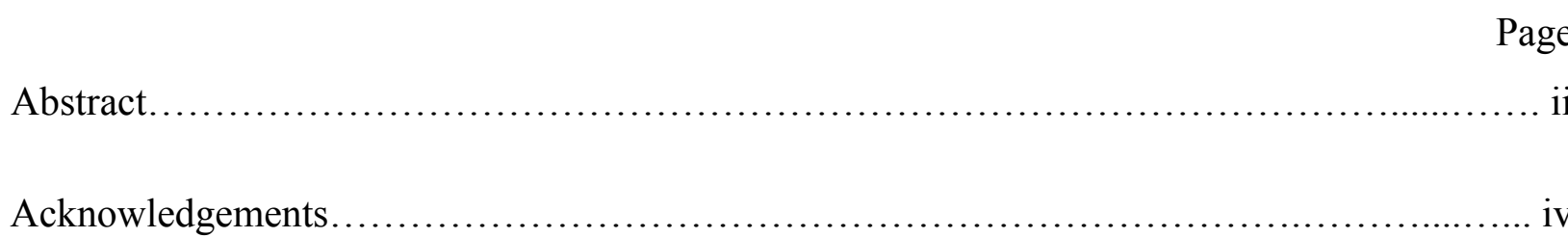

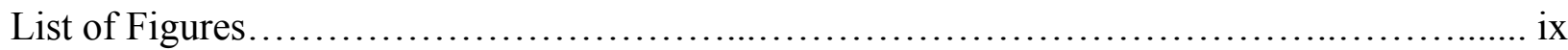

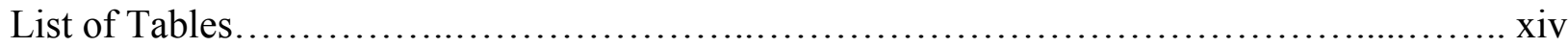

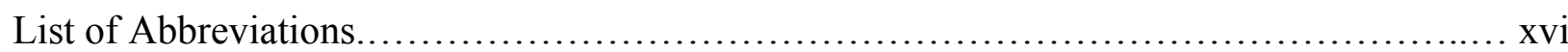

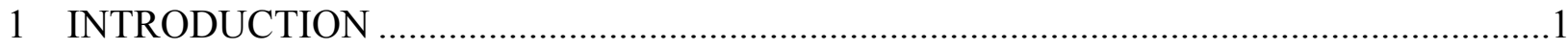

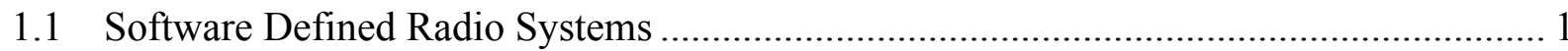

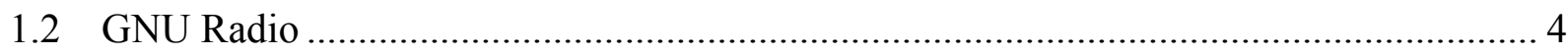

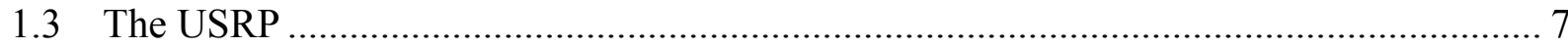

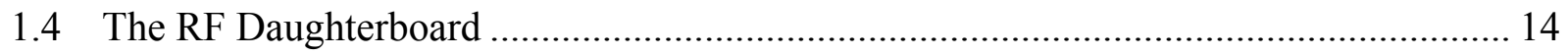

1.5 Literature and Documentation - USRP and GNU Radio ........................................ 17

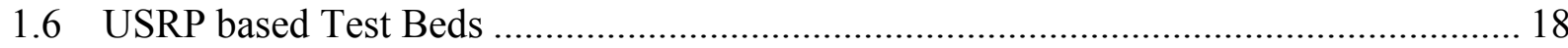

1.6.1 CORNET Test Bed .................................................................................. 19

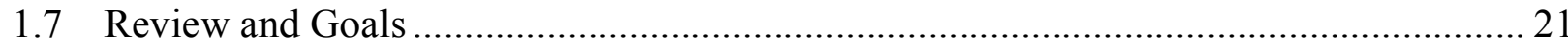

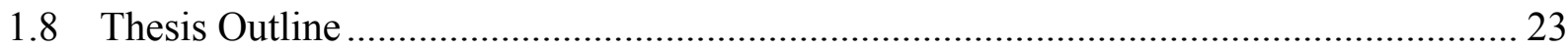

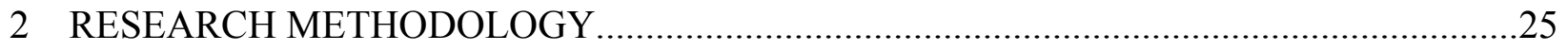

2.1 Calibration of USRPs - Hardware and Software................................................... 25 
2.2 Practical Application of Calibration Factors

2.2.1 Basic Propagation Mechanism....................................................................... 32

2.2.2 Multi Floor Path Loss using CORNET ............................................................. 35

2.2.3 Position Location using Received Signal Strength ......................................... 37

3 EXPERIMENTAL SETUP AND METHODOLOGY .....................................................45

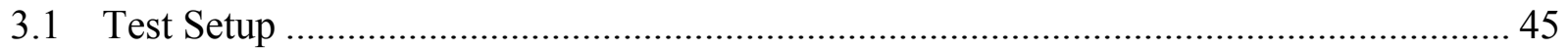

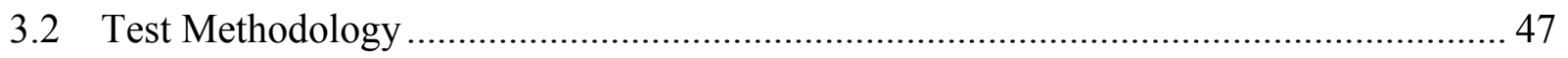

3.2.1 The 1-dB Compression Point Test ................................................................. 49

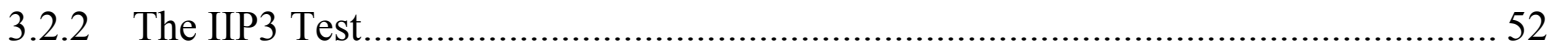

3.2.3 The Received Power Stability Test .................................................................. 57

3.2.4 The Transmitted Signal RF Carrier Frequency Stability Test .............................59

3.2.5 The Transmitted Signal Output Amplitude Stability Test ................................. 62

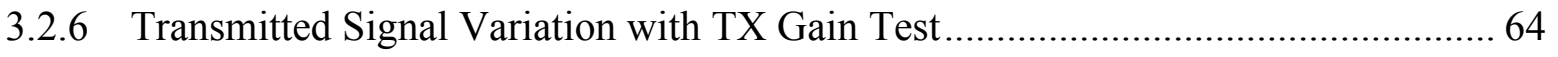

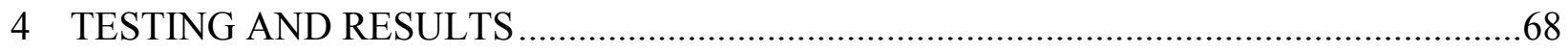

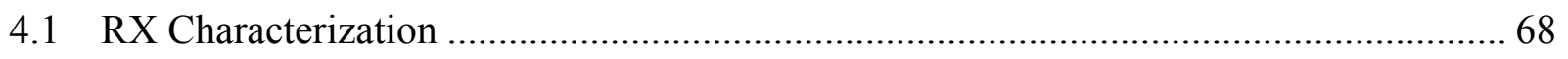

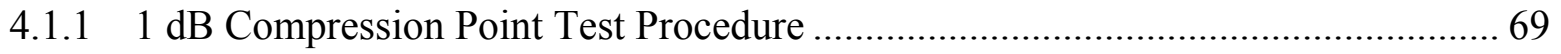

4.1.1.1 Main Results/Observations from 1-dBCP Test.......................................... 72

4.1.2 Received Power Stability Test Procedure ................................................... 73

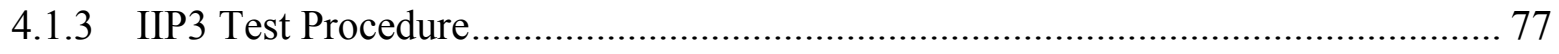




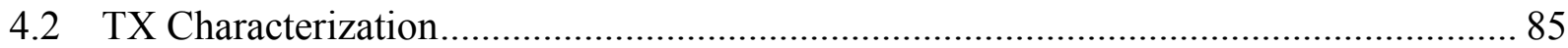

4.2.1 Transmitted Signal Frequency Stability Test Procedure ………………………….. 88

4.2.1.1 Main Results/Observations from TX Frequency Stability Test......................... 90

4.2.2 Transmitted Amplitude Stability Test Procedure ………….................................... 94

4.2.3 Transmitted Signal Power Variation with TX Gain ............................................... 100

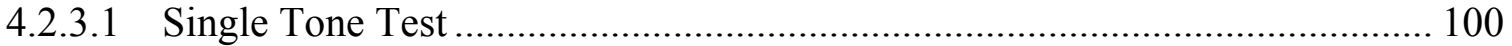

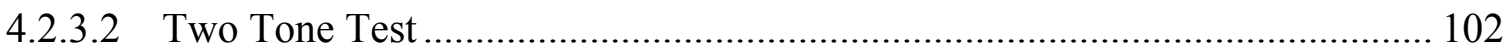

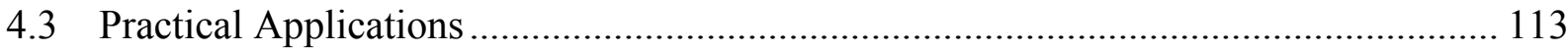

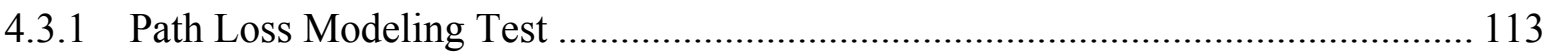

4.3.2 Position Location Estimation Test ....................................................................... 117

4.3.2.1.1 Position Location Experiment Results.................................................... 120

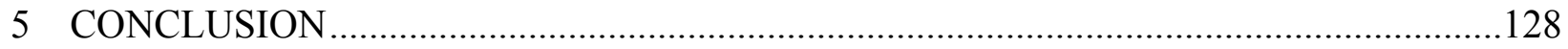

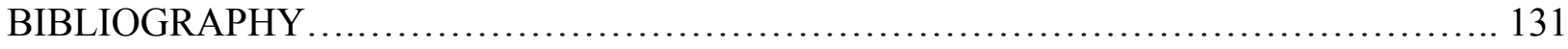

APPENDIX A. Position Location Simulation …....................................... 136

APPENDIX B. Permission from Matt Ettus ......................................... 142 


\section{LIST OF FIGURES}

Page

Figure 1.1 Block Diagram representing SDR Operation 2

Figure 1.2 Sample Flow Graph designed using GRC, (C) Matt Ettus. Used with permission. See Appendix B. Permission from Matt Ettus 6

Figure 1.3 Block Diagram representing Basic TX and RX Operation of USRP Device............. 10

Figure 1.4 Internal Layout of USPRN210, (C) Matt Ettus. Used with permission. See Appendix B. Permission from Matt Ettus 13

Figure 1.5 Block Diagram representing Receive Operation of Daughter Board.... 15

Figure 1.6 Block Diagram representing Transmit Operation of Daughter Board 16

Figure 1.7 CORNET Test-bed (3-D image of the nodes across 4 floors) 20

Figure 2.1 USRPN210 Device, (C) Matt Ettus. Used with permission. See Appendix B. Permission from Matt Ettus 27

Figure 2.2 Flow Graph emulating Real Time Spectrum Analyzer, C Matt Ettus. Used with permission. See Appendix B. Permission from Matt Ettus 28

Figure 2.3 Sample Output of Spectrum Analyzer Utility 29

Figure 2.4 Channel Power Estimation Flow Graph for Position Location Experiments 42

Figure 2.5 Sample Output of Channel Power Estimation Flow Graph 43 
Figure 3.1 Block Diagram of Basic Experimental Setup for RX Tests

Figure 3.2 Laboratory Setup for RX Characterization................................................................... 46

Figure 3.3 Standard I/O Equipment used for the Experiments ................................................... 48

Figure 3.4 Block Diagram of 1-dB Compression Point Test..................................................... 50

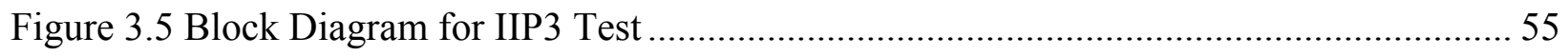

Figure 3.6 Block Diagram of Received Power Stability Test.................................................... 58

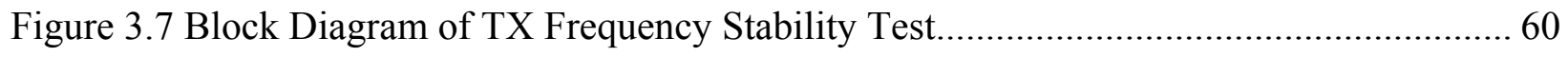

Figure 3.8 Block Diagram of TX Amplitude Stability Test ..................................................... 63

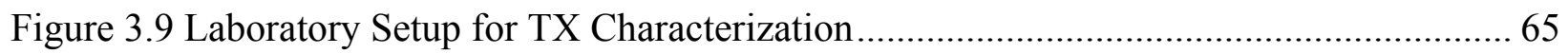

Figure 4.1 Laboratory Setup for 1-dB Compression Point and RX Power Stability Test ............ 69

Figure 4.2 Received Power Vs Input Signal Power (USRPN210-WBX DB) ............................. 73

Figure 4.3 Received Power Vs Frequency (USRPN210-WBX DB) ......................................... 75

Figure 4.4 Received Power Vs Frequency (USRPN210-SBX DB) ............................................ 75

Figure 4.5 Received Power Variation with Frequency @ -30 dBm Input Power .......................... 76

Figure 4.6 Received Power Variation with Frequency @ -60 dBm Input Power ......................... 76

Figure 4.7 Received Power Vs Frequency (USRP2-WBX DB)................................................ 77 
Figure 4.8 Laboratory Setup for IIP3 Test. 79

Figure 4.9 IIP3: Calibrated power (USRPN210-WBX DB) @ 400 MHz...................................... 82

Figure 4.10 IIP3: Un-calibrated power (USRPN210-WBX DB) @ $400 \mathrm{MHz}$............................. 82

Figure 4.11 IIP3: Calibrated Power (USRPN210-WBX DB) @ $900 \mathrm{MHz}$................................. 84

Figure 4.12 IIP3: Un-calibrated Power (USRPN210-WBX DB) @ $900 \mathrm{MHz}$............................. 84

Figure 4.13 Laboratory Setup for TX Frequency and TX Power Stability Test........................... 87

Figure 4.14 Transmitted Frequency Stability Test (USRPN210-WBX DB)............................... 91

Figure 4.15 Transmitted Frequency Stability Test (USRP2-WBX DB)…………………........ 92

Figure 4.16 Transmitted Frequency Stability Test (USRPN210-SBX DB) ................................ 92

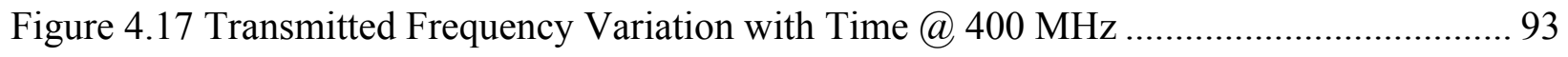

Figure 4.18 Transmitted Frequency Variation with Time @ $900 \mathrm{MHz}$...................................... 93

Figure 4.19 Transmitted Frequency Variation with Time @ $1800 \mathrm{MHz}$.................................... 94

Figure 4.20 Transmitted Power Stability Test (USRPN210-WBX DB) ……………….............. 97

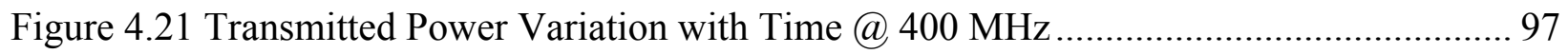

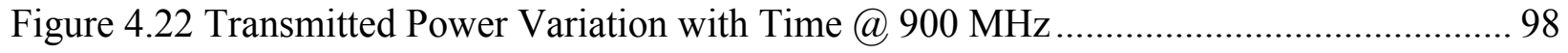

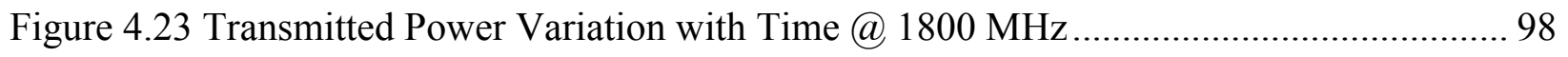


Figure 4.24 Transmitted Power Stability Test (USRPN210-SBX DB) 99

Figure 4.25 Transmitted Power Stability Test (Custom Flow Graph, USRPN210-WBX DB).... 99

Figure 4.26 Laboratory Setup for TX Power Variation with TX Gain Test 100

Figure 4.27 Transmitted Power Variation with TX Gain (USRPN210-WBX DB) 106

Figure 4.28 F1 Power Variation with TX Gain, Two Tone Test (USRPN210-WBX DB) ........ 108

Figure 4.29 Relative IMD Power Variation with TX Gain (USRPN210-WBX DB)................. 108

Figure 4.30 Transmitted Power Variation with TX Gain (USRPN210-SBX DB) 109

Figure 4.31 F1 Power Variation with TX Gain, Two Tone Test (USRPN210-SBX DB) 109

Figure 4.32 Relative IMD Power Variation with TX Gain (USRPN210-SBX DB) 110

Figure 4.33 Transmitted Power Variation with TX Gain (USRPN2-WBX DB) 110

Figure 4.34 F1 Power Variation with TX Gain, Two Tone Test (USRPN2-WBX DB) 111

Figure 4.35 Relative IMD Power Variation with TX Gain (USRP2-WBX DB). 111

Figure 4.36 Layout of Room Used for Position Location Experiment 121

Figure 4.37 Position of the Receiver Nodes w.r.t Transmitter in Large Room ... 122

Figure 4.38 Layout of Corridor used for Position Location Experiment 123

Figure 4.39 Position of Receiver Nodes w.r.t TX in Corridor, Exp 1 124 
Figure 4.40 Position of Receiver Nodes w.r.t TX in Corridor, Exp 2

Figure 4.41 Variation of Received Power with Distance 125 


\section{LIST OF TABLES}

Page

Table 1.1 Comparison of different USRP Device Series from Ettus Research, (C) Matt Ettus. Used with permission. See Appendix B. Permission from Matt Ettus

Table 2.1 USRPN210 Specifications, (C) Matt Ettus. Used with permission. See Appendix B.

Permission from Matt Ettus 26

Table 3.1 Correction Factors for different USRP Devices across different Frequencies 51

Table 3.2 Analytical IIP3 Calculation at different Frequencies 56

Table 3.3 Amplitude Change across different Devices and Frequencies steps 59

Table 3.4 Transmitted Signal Frequency Stability Test Measurements

61

Table 3.5 Transmitted Signal Amplitude Stability Test Measurements 64

Table 3.6 Transmitter Signal Variation with TX Gain Test Measurements 66

Table 4.1 Readings for 1-dBCP Test (Correction Factors derived)..... 71

Table 4.2 Readings for Received Power Stability Test 74

Table 4.3 Readings for IIP3 Test @, $400 \mathrm{MHz}$ 81

Table 4.4 Readings for IIP3 Test @ 900 MHz 83

Table 4.5 IIP3 Analytically Calculated @ 400 MHz (USRPN210-WBX DB)..... 85 
Table 4.6 IIP3 Analytically Calculated @ 900 MHz (USRPN210-WBX) 86

Table 4.7 Readings for Transmitted Frequency Stability Test 89

Table 4.8 Readings for Transmitted Power Stability Test 96

Table 4.9 Readings for Transmitted Power Variation with Gain Test, Single Tone 105

Table 4.10 Readings for Transmitted Power Variation with Gain Test, Two Tone 107

Table 4.11 Power Reference Table: Transmitted Power Variation with TX Gain 112

Table 4.12 Path Loss Calculation with Calibrated Power using CORNET 115

Table 4.13 Path Loss Calculation with Un-calibrated Power using CORNET 116

Table 4.14 Floor Attenuation Factor Calculation with Calibrated Power 117

Table 4.15 Root Mean Square Error: Pos. Est. in Large Room 122

Table 4.16 Root Mean Square Error: Pos. Est. in Corridor 124

Table 4.17 Root Mean Square Error: Pos. Est. in Corridor (3 nodes, QT Flow Graph) 126

Table 4.18 Root Mean Square Error: Pos. Est. in Corridor (5 nodes, QT Flow Graph). 126

Table 4.19 Root Mean Square Error Variation with Path Loss Exponent 127 


\section{LIST OF ABBREVIATIONS}

\begin{tabular}{|l|l|}
\hline Acronym & Term Meaning \\
\hline ADC & Analog to Digital Converter \\
\hline API & Application Programming Interface \\
\hline CORNET & Cognitive Radio Network \\
\hline DAC & Digital to Analog Converter \\
\hline FFT & Fast Fourier Transform \\
\hline FPGA & Field Programmable Gate Array \\
\hline GPS & Global Positioning System \\
\hline GPSDO & GPS Disciplined Oscillator \\
\hline GRC & GNU Radio Companion \\
\hline GUI & Graphical User Interface \\
\hline IF & Intermediate Frequency \\
\hline IIP3 & Third Order Input Intercept Point \\
\hline IMD & Inter-Modulation Distortion \\
\hline IQ & In-phase Quadrature \\
\hline MIMO & Multiple Input Multiple Output \\
\hline OIP3 & Third Order Output Intercept Point \\
\hline OS & Operating System \\
\hline PLL & Phased Locked Loops \\
\hline PPM & Parts Per Million \\
\hline RBW & Resolution Bandwidth \\
\hline RF & Radio Frequency \\
\hline SBX & RF Daughterboard of USRP, Frequency Range: $400 \mathrm{MHz} \sim 4.4 \mathrm{GHz}$ \\
\hline SDR & Software-Defined Radio \\
\hline UHD & USRP Hardware Driver \\
\hline USRP & Universal Software Radio Peripheral \\
\hline USRPNXXX & USRP Network Series, \\
\hline USRPBXXX & USRP Bus Series \\
\hline USRPEXXX & USRP Embedded Series \\
\hline USRPXXXX & USRP X-Series \\
\hline VCO & Voltage Controlled Oscillator \\
\hline WBX & RF Daughterboard of USRP, Frequency Range: DC $\sim 4.4 \mathrm{GHz}$ \\
\hline & \\
\hline
\end{tabular}




\section{INTRODUCTION}

The progressive research in radio communication focusing on software radio area has helped improve RF design in digital and analog domain along with the implementation techniques. This chapter begins by providing an overview of the Software Defined Radio systems, GNU Radio Software utility followed by the USRP hardware configuration and working and finally ending with the description of commonly used transceiver daughter board configurations. A brief note of the need and importance of calibration of USRPs is also provided and discussed in reference practical applications like path loss modeling and position location estimation.

In this chapter, the supporting literature that motivates and complements the research is summarized. Finally the scope of this thesis is discussed.

\subsection{Software Defined Radio Systems}

A radio is a device which transmits and receives signals using the allowed radio frequency bandwidth in the electromagnetic spectrum to transfer information wirelessly. Earlier the spectrum utilization was limited due to many restrictions imposed by the hardware analog circuitry, signal processing capabilities and limited consumers. Technological advancements have not only led to increased spectrum utilization but also increase in the needs of the end users. With various modes of communications necessitated today such as voice, data, video communications, broadcast messaging, control and emergency response communications, it is becomes even more critical for businesses to be able to modify the radio features easily and costeffectively. This is facilitated by Software Defined radio by means of reconfigurable firmware. 
SDR has some or most of the physical layer processing defined in software. The field of software-defined radios is fast growing and the on-going developments in this field are enormous and remarkable. For example SDR systems are commonly used in Cellular communications wherein utilizing the System on Chip (SoC) devices incorporating the "DSP Cores" the handsets support baseband and modem processing. Using the SDR functionality the base stations are also enabled with multi-band and multi-protocol support functionality. The figure below illustrates the basic concept of software-defined radio.

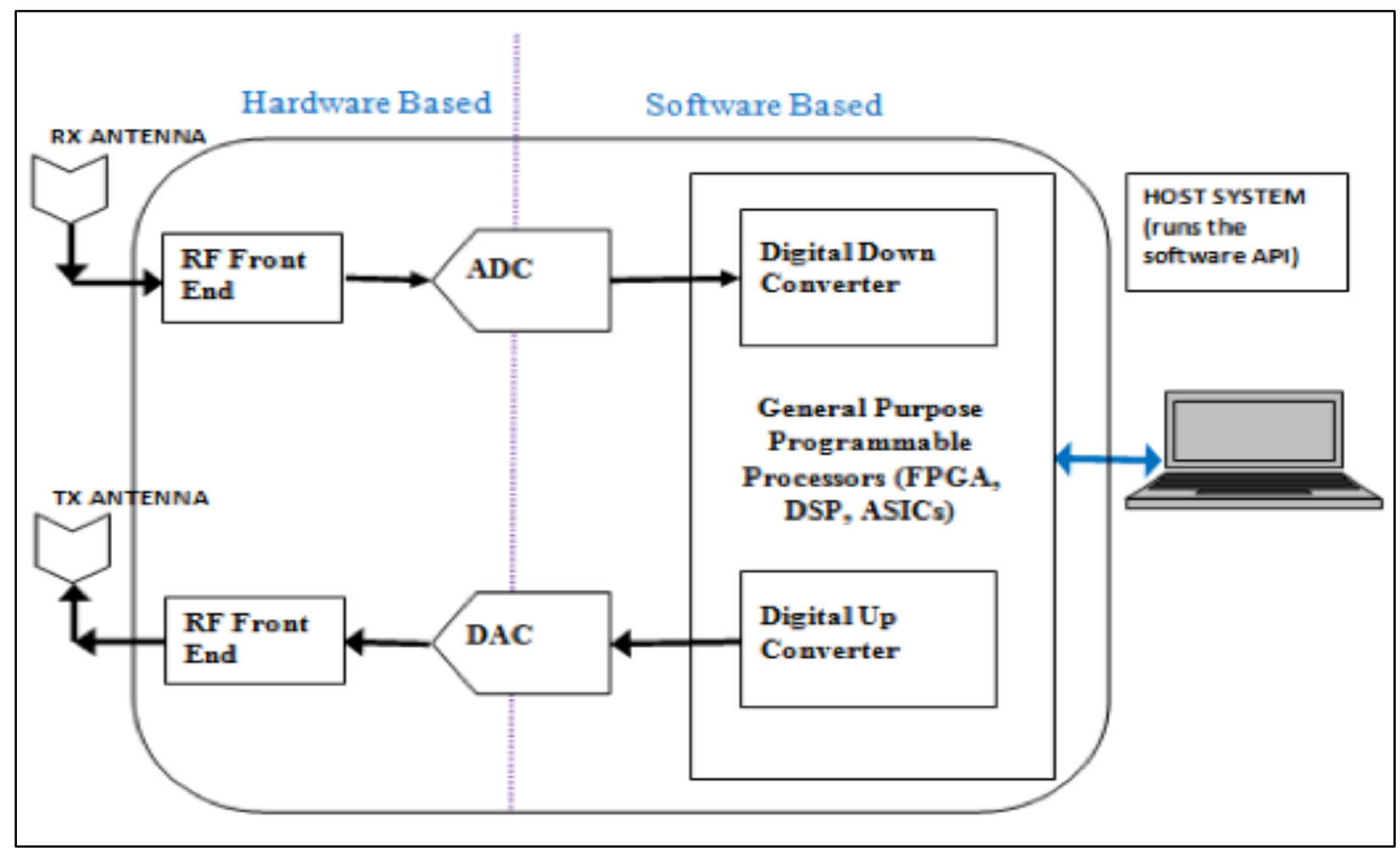

Figure 1.1 Block Diagram representing SDR Operation 
Traditional hardware based radio systems were not easily modified and had to done through explicit replacement of the component. This made the hardware based radio system to be usable only for a set band of frequencies and protocol set. However with software defined radios, having most of their processing defined in easily re-programmable components like General purpose Processors (GPP), Field Programmable Gate Arrays (FPGA), System on Chip (SoC) provides flexibility in terms of multi-band, multi-mode functionality. They can be easily reconfigured to support different wireless protocols. E.g., A single USRP device can be used as a FM receiver or used to observe the GSM downlink channel by just replacing the associated program code/flow graph in the GNU Radio interface [1]. They also provide cost effective means of adding new functionality or capabilities to already existing radio systems without change in hardware by means of software upgrades.

SDR systems are beneficial for equipment manufacturers, radio service providers and end users. In this respect some of the advantages of SDR can be said to be flexible framework (reconfigurable firmware interfaces, allow development of common platform for set of radio products, reusable software allowing lower development costs), easy to implement different radio protocols (different modulation schemes, or even different wireless technologies) and also easily integrated with existing infrastructure, low cost and portable. They also incorporate greater interference rejection capability (by means of smart antennas), allow cooperative spectrum utilization (using cognitive radios) and network management facility (via dynamic transmit power adjustment capability to avoid near-far problems). 
Software defined radio has created a huge impact in wireless communications and are now used in military applications, mobile communications, public safety radios and are very useful for research and prototype testing purposes too.

Commonly used laboratory hardware devices are the USRP Series from Ettus Research, RTL-SDR, and FLEX SDR series. These would mostly consist of motherboard where majority of functionality would be concentrated. Some devices have additional daughter boards with the RF functionality. These SDR devices need to be connected to a host system for enabling and monitoring their functionality. This is mainly done through application processing interfaces like GNU Radio, Simulink, and LabView. In this thesis, USRP devices and GNU Radio software interface combination was used for hence the same been described in following sections in this chapter.

\subsection{GNU Radio}

GNU Radio is an open-source, free software package for software-defined radio design and development. GNU Radio is often run on a host system in conjunction with UHD (Universal Hardware Drivers) for implementing functions of a radio with the USRP (universal Software Radio Peripheral). The details of the GNU Radio package are available at the GNU Radio website [2]. The system requirements for running GNU Radio are simple; it can be run on a standard laptop/PC with sufficient memory. GNU Radio is popularly run on LINUX OS though MAC and Windows OS support it as well. Thus GNU Radio is a simple easy to use software package, which allows anyone with basic knowledge of computers and with little or no programming experience also to develop flow graphs and implement software radio principles. 
GNU Radio is typically written in $\mathrm{C} / \mathrm{C}++$ language at the low-level and wrapped up in Python on a high-level. This is because $\mathrm{C} / \mathrm{C}++$ allows faster processing while Python allows a easier user interface for understanding and modification of code at the high level [3].

The GNU Radio toolkit also provides easy to use graphical user interface - GNU Radio Companion (GRC) [2] which allows a user to develop a flow graph for signal processing by using the pre-defined blocks for signal source/sinks, FFT processing, interpolation/decimation and modulation/demodulation etc. A developer proficient in $\mathrm{C} / \mathrm{C}++$ or Python can easily modify these blocks as per his/her requirements in order to form a custom-made flow graph. The transmitter outputs the data as complex baseband samples and the receiver inputs the same type of data. The interfacing hardware such as USRP devices are used to shift the data to analog domain at the RF carrier frequency. Apart from this requirement all the data samples can be processed in form of bit/byte streams, vectors, packet bursts or complex I-Q data [2].

The hardware used as front end is usually the limiting factor for the allowed range of signal processing functions. The system processor memory and speed are other factors, which limits the processing capabilities of the signal processing blocks in the flow graphs. Another factor to consider is that the front-end hardware can either be used as half /full duplex processing. Which again would restrict the functionality in terms of signal bandwidth, utilizable frequency spectrum, transmitting power, dynamic range and minimum detectable signal [4].

GNU Radio helps implement many features of the software radio like filters, modulators, phase-locked loops etc. The package provides these features as different signal processing blocks, which can be connected together to form a cohesive flow graphs. A simple example flow graph is shown below. 


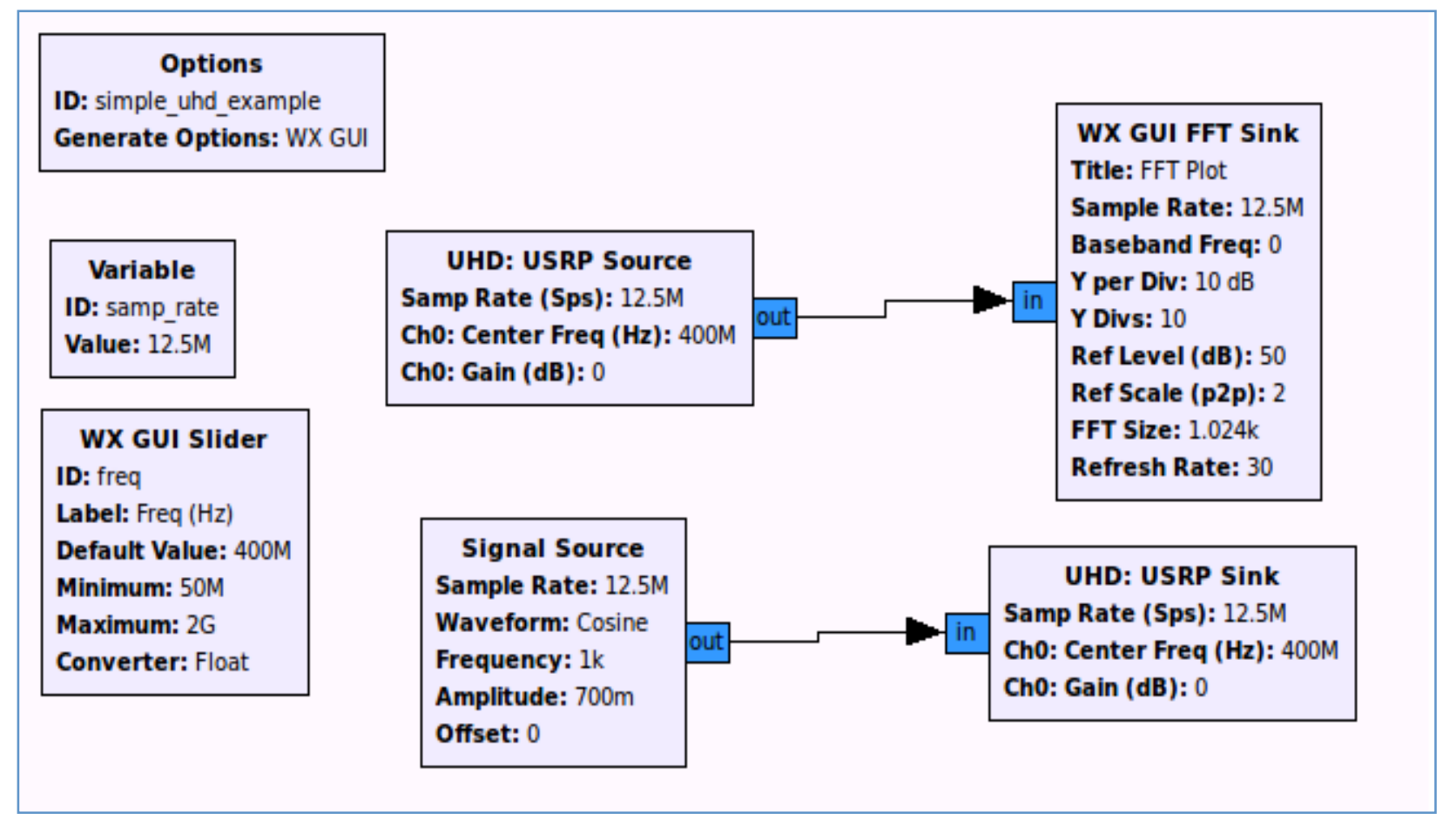

Figure 1.2 Sample Flow Graph designed using GRC, (C) Matt Ettus. Used with permission. See

Appendix B. Permission from Matt Ettus

A sample flow graph has been shown in Figure 1.2 representing simple signal processing flow [5]. This flow graph consists only of 2 sub flow graphs each with 2 signal-processing blocks: a data source and a sink. The components are interconnected by means of Input-output ports. However the first block in each of the sub-flow graphs has only an output port and acts a source of digital samples (either from a USRP device behaving as a RX i.e. UHD_USRP Source or a signal source block of GRC). When using UHD_USRP_SOURCE block, the connected USRP device acts as a Receiver and may be either connected to an external signal generator or to an antenna for receiving the RF signals. The second block in both sub-flow graphs consists only of an input port signifying that it receives the samples and processes it at the end as required. 
Thus the flow of data would be from left to right on flow graph execution, and each component has to be set with the required parameters such as the Source depends on center frequency, sampling rate and amplification. The sink is set at the RF frequency of the desired Rx signal and in case of WX_FFT_GUI block the correct RX port for reception also needs to be selected. When the run button is hit, the flow graph is connected and data starts data transmission using the parameters' values set in the blocks. For e.g. when the connected USRP is to behave as a receiver, the baseband digital signal samples are generated from the external signal source and the samples are sent to the USRP sink (WX_FFT_GUI which behaves as a spectrum analyzer).

In summary it can be said that in GNU Radio flow graphs are means of signal processing and every flow graph consists of interconnected blocks that perform operations like filtering, adding/multiplexing, transforming, decoding signals, allowing hardware access etc. Data is passed in forms of complex or real data types and every flow graph needs at least one source or $\operatorname{sink}[1]$.

\subsection{The USRP}

Universal software radio peripheral or USRP refers to a line of RF front end products made by Ettus Research, and is popularly used by researchers, hobbyists for implementing the software defined radios. USRPS are often used with GNU Radio, but can be used with other software too using the UHD software package API. The USRP device is much less expensive than many laboratory instruments, and the original USRP schematic details as well as the compatible software are freely available on the Internet as open-source [2] [6].

The main board of the USRP device, called "motherboard" is the main interface to the host computer and provides basic functionality of signal processing like frequency down/up 
conversion, $\mathrm{A} / \mathrm{D}$ and $\mathrm{D} / \mathrm{A}$ processing and interpolation and decimation. USRPs also allow connection to one or more "daughter boards" which can be plugged into the motherboard to complete the RF-front end. The daughter boards perform analog processing like amplification, filtering and conversion to baseband [6].

The USRP has two ports for connecting the signal path to the daughter boards. The daughter boards can either function as Transmit only (TX) or Receive Only (Rx) or as transceiver boards based on the connection. The daughter boards pass the signal from antenna to main board or vice-a-versa.

Ettus Research has brought out different series of USRP devices with enhanced capabilities to cater to specific applications. The USRPN2XX (Network) series is suited for applications like prototyping, dynamic spectrum access, cognitive radio and sensor networks requiring high RF performance, large dynamic range and bandwidth [6]. It also enables MIMO configuration implementation by connecting two USRPN2XX devices together using a MIMO cable and synchronizing their clocks for proper functioning. The USRPBXX (Bus) series is a fully integrated single board SDR device useful for a wide range of applications such as FM broadcast, cellular, GPS, Wi-Fi, ISM and more. The USRPEXX (Embedded) series allows unmanned operations for embedded applications and for those that do not require full host CPU processing ability, and is ideal for mobile applications such as stand-alone base stations. The main components details for various series are tabulated in Table 1.1 below [7]. 
Table 1.1 Comparison of different USRP Device Series from Ettus Research, (C) Matt Ettus. Used with permission. See Appendix B. Permission from Matt Ettus

\begin{tabular}{|c|c|c|c|c|c|}
\hline Specifics & USRP2 & USRPN10 & USRPB200/210 & USRPE100/110 & USRPX300/310 \\
\hline Interface & $\begin{array}{l}\text { Gigabit } \\
\text { Ethernet }\end{array}$ & $\begin{array}{c}\text { Gigabit } \\
\text { Ethernet, } \\
\text { Auxiliary } \\
\text { Analog \& } \\
\text { Digital I/O }\end{array}$ & USB 3.0 & $\begin{array}{l}\text { Embedded, } \\
\text { Stereo I/O, } \\
\text { HMI out, USB } \\
\text { Host, USB } \\
\text { OTG, Ethernet } \\
\text { \& Serial } \\
\text { console }\end{array}$ & $\begin{array}{c}\text { Dual SFP }(+) \\
\text { port for 1/ } 10 \\
\text { Gigabit } \\
\text { Ethernet, PCIe } \\
\text { X4, }\end{array}$ \\
\hline $\begin{array}{l}\text { Host Sample } \\
\text { Rate }\end{array}$ & $\begin{array}{c}25 \text { MSps @ } \\
16 \text { bit }\end{array}$ & $\begin{array}{c}25 \text { MSps @ } \\
16 \text { bit }\end{array}$ & $\begin{array}{c}61.44 \text { MSps @ } \\
16 \text { bits }\end{array}$ & 4 MSps & $\begin{array}{c}200 \mathrm{MSps} @ 16 \\
\text { bits }\end{array}$ \\
\hline FPGA & $\begin{array}{c}\text { Xilinx } \\
\text { Spartan 3- } \\
\text { 2000 FPGA, }\end{array}$ & $\begin{array}{c}\text { Xilinx } \\
\text { Spartan 3A- } \\
\text { DSP, 3400 } \\
\text { FPGA, } \\
\text { onboard } \\
\text { processing }\end{array}$ & $\begin{array}{c}\text { Spartan 6 } \\
\text { XC6SLX150 }\end{array}$ & $\begin{array}{c}\text { Xilinx Spartan } \\
\text { 3A-DSP, } 1800 \\
\text { FPGA }\end{array}$ & $\begin{array}{c}\text { Xilinx Kintex- } 7 \\
\text { FPGA } \\
\text { (XC7K325T \& } \\
\text { XC7K410T) }\end{array}$ \\
\hline $\mathrm{ADC}$ & $\begin{array}{l}\text { 14-bit, } 100 \\
\text { MSps }\end{array}$ & $\begin{array}{c}\text { 14-bit, } 100 \\
\text { MSps }\end{array}$ & $\begin{array}{c}\text { 12-bit, } 61.44 \\
\text { MSps }\end{array}$ & $\begin{array}{c}\text { 12- bit, } 64 \\
\text { MSps }\end{array}$ & $\begin{array}{c}\text { 14-bit, } 200 \\
\text { MSps }\end{array}$ \\
\hline $\mathrm{DAC}$ & $\begin{array}{c}\text { 16-bit, } 400 \\
\text { MSps }\end{array}$ & $\begin{array}{c}\text { 16-bit, } 400 \\
\text { MSps }\end{array}$ & $\begin{array}{c}\text { 12-bit, } 61.44 \\
\text { MSps }\end{array}$ & $\begin{array}{c}\text { 14- bit, } 128 \\
\text { MSps }\end{array}$ & $\begin{array}{c}\text { 16-bit, } 800 \\
\text { MSps }\end{array}$ \\
\hline $\begin{array}{l}\text { Frequency } \\
\text { Accuracy }\end{array}$ & & $\begin{array}{c}\sim 2.5 \mathrm{ppm} \\
\text { with onboard } \\
\text { clock } \\
\text { reference, } \\
0.01 \mathrm{ppm} \\
\text { with GPSDO } \\
\text { reference }\end{array}$ & $\begin{array}{l} \pm 2 \text { ppm, } \pm 75 \\
\text { ppb with GPS } \\
\text { unlocked } \\
\text { TCXO } \\
\text { reference }\end{array}$ & $\begin{array}{c}2.5 \text { ppm with } \\
\text { onboard clock } \\
\text { reference, } 0.01 \\
\text { ppm with } \\
\text { GPSDO } \\
\text { reference }\end{array}$ & $\begin{array}{c}2.5 \text { ppm with } \\
\text { onboard clock } \\
\text { reference, } 20 \\
\text { ppb with } \\
\text { GPSDO } \\
\text { reference }\end{array}$ \\
\hline $\begin{array}{l}\text { Storage } \\
\text { Configuration }\end{array}$ & $\begin{array}{l}\text { SRAM, } 1 \\
\text { Megabyte }\end{array}$ & $\begin{array}{l}\text { SRAM, } 1 \\
\text { Megabyte }\end{array}$ & Not specified & $\begin{array}{l}512 \text { MB RAM } \\
\text { /4 GB Flash }\end{array}$ & $\overline{\mathrm{N} / \mathrm{A}}$ \\
\hline Operation & Stand alone & $\begin{array}{l}\text { Networked } \\
\text { series/stand } \\
\text { alone }\end{array}$ & $\begin{array}{c}\text { Stand-alone for } \\
\text { open BTS }\end{array}$ & $\begin{array}{l}\text { Stand-alone for } \\
\text { embedded } \\
\text { applications }\end{array}$ & Stand-alone \\
\hline $\begin{array}{l}\text { Multiple } \\
\text { system } \\
\text { capability }\end{array}$ & $\begin{array}{c}\text { MIMO with } \\
2 \text { or more } \\
\text { systems }\end{array}$ & $\begin{array}{l}\text { MIMO with } \\
2 \text { or more } \\
\text { systems }\end{array}$ & 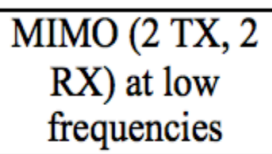 & Not specified & $\begin{array}{l}\text { Massive MIMO } \\
\text { Test beds }\end{array}$ \\
\hline $\begin{array}{l}\text { Daughter } \\
\text { Board Capacity }\end{array}$ & $\begin{array}{c}1 \mathrm{TX} / 1 \mathrm{RX} \\
\text { or } 1 \\
\text { transceiver }\end{array}$ & $\begin{array}{c}1 \mathrm{TX} / 1 \mathrm{RX} \\
\text { or } 1 \\
\text { transceiver }\end{array}$ & $\begin{array}{c}\text { N/A (A single } \\
\text { integrated board } \\
\text { with full duplex } \\
\text { ability) }\end{array}$ & $\begin{array}{l}1 \mathrm{TX} / 1 \mathrm{RX} \text { or } \\
1 \text { transceiver }\end{array}$ & $\begin{array}{c}2 \text { wideband RF } \\
\text { boards }\end{array}$ \\
\hline
\end{tabular}


The main components of USRP are shown in Figure 1.3 [6]. The DC port and gigabit Ethernet cable port are at the bottom of the board. The middle of the board contains the FPGA with the DACs and ADCs on either side. Only one Daughter board is connected to the motherboard with RF1 port allowing TX and Rx functionality and RF2 allowing RX functionality alone. These ports may have antenna connection or wired connection via SMA connectors.

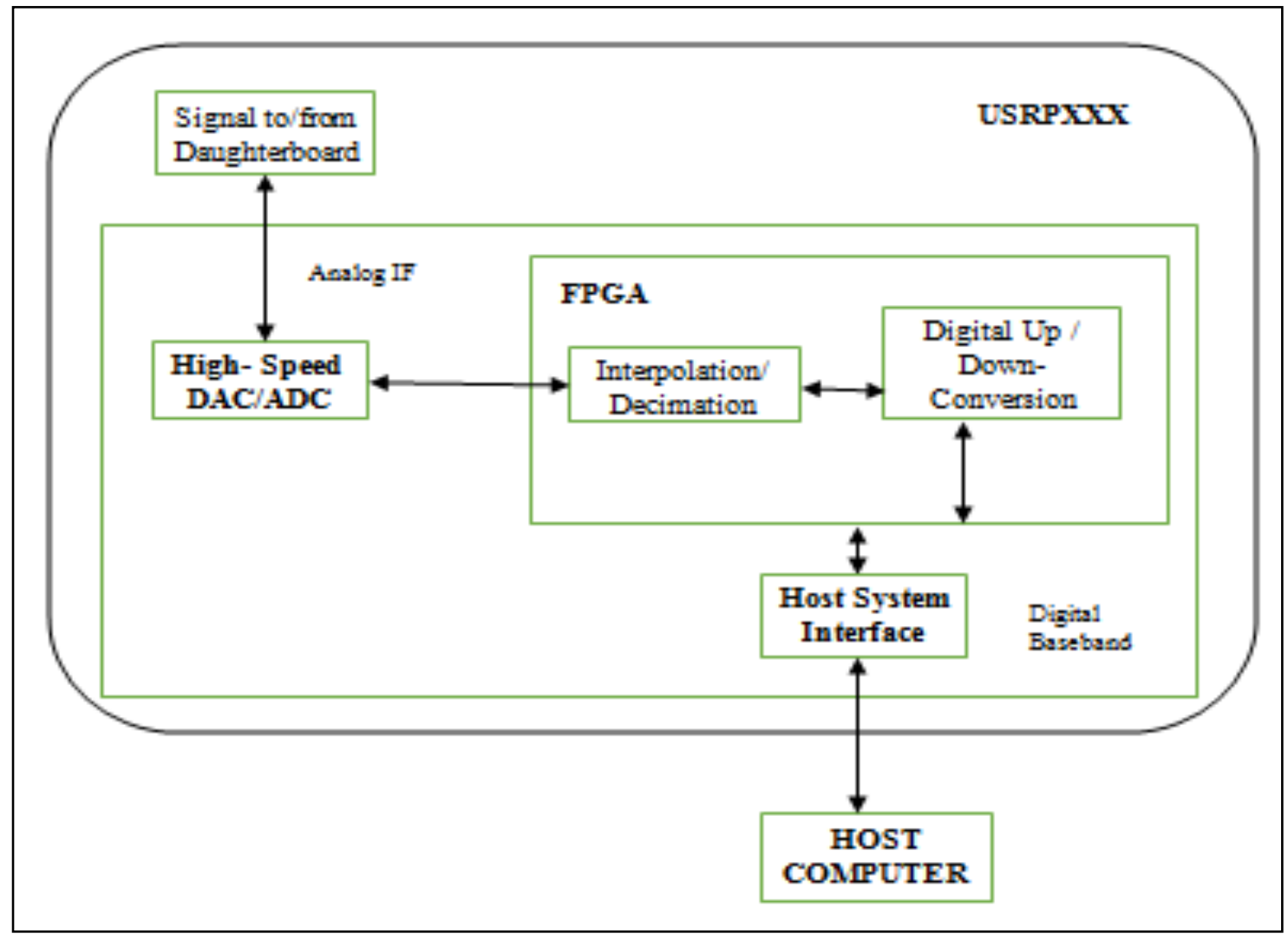

Figure 1.3 Block Diagram representing Basic TX and RX Operation of USRP Device 
Figure 1.3 shows a general internal layout of the USRP device [6]. On the Receive operation, the analog signal at radio frequency is received by daughter board via RX (RF2) port, which in turn is tuned to the desired frequency of operation. The daughter board operates on the signal in terms of filtering, amplification and down converts it to an Intermediate frequency. It can either be in terms of base band signal $(0 \mathrm{~Hz})$ or also as single or quadrature signals. The ADC digitizes the signal, and sends it to FPGA where the first stage of decimation occurs in order to lower the signal's sampling rate depending upon the requirements of data rate and precision [6]. If received signal is at IF and not quadrature, it is now down-converted to baseband frequency and made as IQ signal (host system can accept signal samples in quadrature form at BB). This signal is sent via host system interface controller to the host computer. GNU Radio or similar software package such as Simulink receives the signal and processes it - filters, demodulates and then process the raw data (plots the received signal's real time spectrum or stores the data for later reference) [2].

While transmitting, the host computer running the GNU Radio package runs the flow graph (that creates the digital samples of the desired RF signal to be transmitted) and sends the digital data via the host system interface controller. This data is typically quadrature in nature output from the modulator depending upon the bandwidth and precision requirements. The FPGA receives the data and turns it into an IF along with interpolation to utilize the high speed DAC. The DAC converts it into an analog signal and send it to the attached daughterboard now functioning as a transmitter. The daughterboard attached inside the USRP may function either at IF or at baseband and/or may require single signal or quadrature signals [6]. The GNU Radio 
driver actually carries this operational requirement of the daughter board and other such configuration information. This operation is also shown in Figure 1.3 [6].

The main connection between host system and USRP hardware is provided by the host system interface controller is the main connection between PC and hardware allowing both sides of communication between host computer and USRP. This interface has also evolved with the hardware and the bandwidth/precision requirements. In the earlier USRP models, this interface was through a USB 2.0 controller while in USRP2 devices it changed to gigabit Ethernet controller. Though the gigabit Ethernet interface was retained for the USRPN series devices, it was enhanced to USB 3.0 interface for USRPB210 devices and Dual 10 gigabit Ethernet for USRPX310 devices.

The USRP embedded series (USRPEXXX) models can also be run as a stand-alone unit by downloading the program onto the device memory from the host computer [4]. Along with the main FPGA, they also include an OMAP 3 (ARM Cortex A8 and C64 DSP), which allow for stand-alone applications without relying much on the host systems computational ability.

The FPGA is main component on the motherboard which is responsible for all the complex computations and also functions like mixing, up/down conversion to/from IF from/to baseband respectively and interpolation/decimation. The IF is then sent to the daughterboard, which converts the IF to the desired carrier frequency and transmits it as analog RF signal. The daughterboard is usually responsible for conversion from IF to RF and RF to IF in transmit and receive operation respectively so as to avoid DC offset issues and other noise sources like I/f noise and shot noise [6]. The USRPX series allow Digital I/O accessible from the front panel for custom control and to interface with the FPGA. 
The USRP's other major computational components are the ADC and DAC chips since the FPGA, GNU Radio and host computer all work with digital samples. In USRPN210 series $\mathrm{ADC}$ runs at $100 \mathrm{MSamples} / \mathrm{sec}$ at a resolution of 14 bit samples, while the DAC runs at 400MSamples/sec at a resolution of 16 bit samples. The ADC converts analog IF to digital IF while DAC converts digital samples at IF to analog IF as the front-end daughter boards work with the analog signals only and are responsible for conversion to/from RF from/to IF. The ADC/DAC chips are integrated with several low speed ADC/DACs that help to monitor signals and control the power levels or frequency deviations etc. Usually the ADC/DAC works on the IQ samples and hence can provide two channels for data streaming to/from the daughter boards [8]. The Schematic internal layout of USRPN210 has been shown below in Figure 1.4 [8], detailing the exact components used and their interconnectivity.

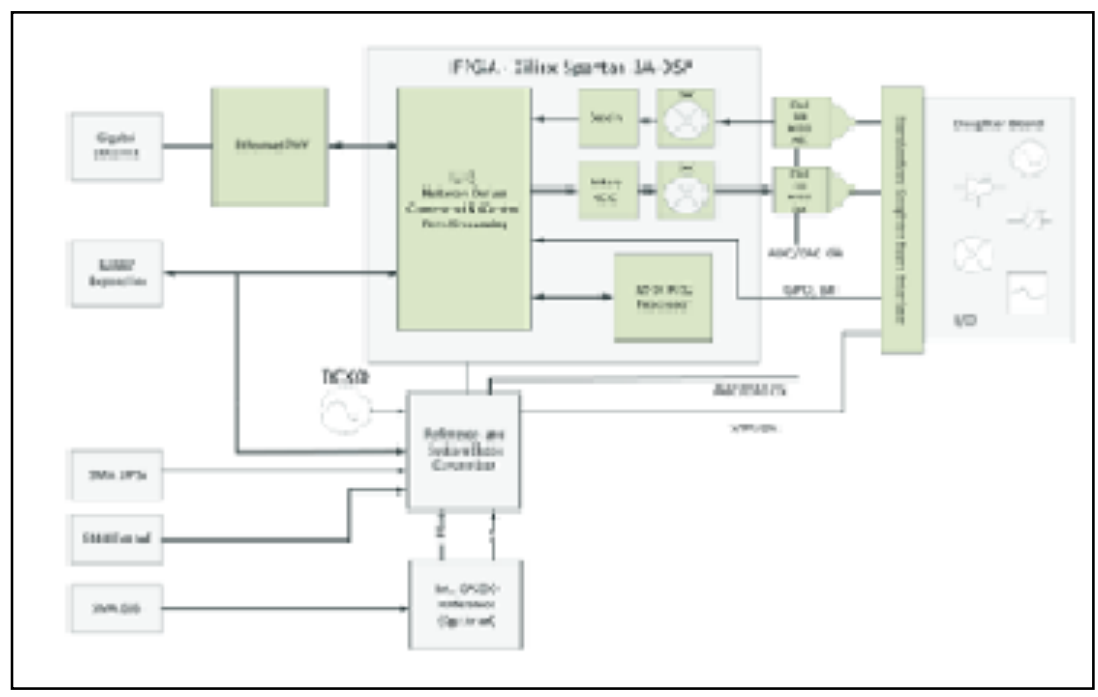

Figure 1.4 Internal Layout of USPRN210, () Matt Ettus. Used with permission. See Appendix B. Permission from Matt Ettus 
The USRPN210 used here for experimentation purposes has many advanced features as compared to earlier versions of USRPs be it with respect to the ADC/DAC computational ability and increased accuracy and data throughput due to the capacity of the advanced FPGA to handle more functions and much improved daughterboard capabilities. The higher RAM memory allows faster computations on board without the need to interface with the host computer. MIMO capabilities are also enabled via SMA cables interface. But USRPN210 allows only 1 daughter board connection at a time. Although this allows for simultaneous TX/Rx operations, it reduces the maximum utility bandwidth of communication available for any connection. However, due to enhanced capabilities USRPN210 is more expensive than its earlier counter parts [6] [8].

\subsection{The RF Daughterboard}

The daughter boards main purpose is to provide a RF front end for transmit and receive operations. They handle functions like amplification and filtering to correctly interface with the antennas. Daughter boards can either provide TX, Rx or even transceiver operations. Receiver daughter boards receive radio signals of desired frequency from the antennas, amplify them and remove the interfering noise by filtering and down convert them to lower IF and send them to ADC present on the motherboard. Similarly transmitter daughter boards take the digital samples from the DAC, up-convert them from IF to RF, filter and amplify them before transmitting them out from the TX antennas. Higher end versions of daughter boards like WBX allow direct conversion from baseband to RF and vice a versa [3]. 


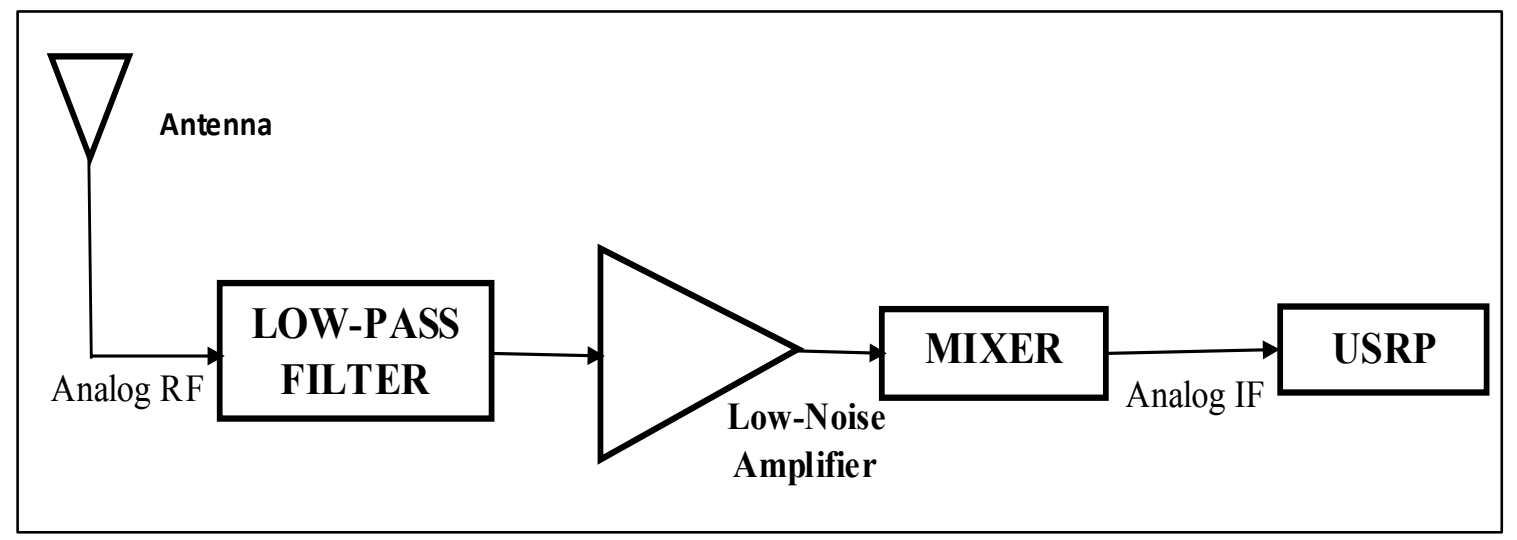

Figure 1.5 Block Diagram representing Receive Operation of Daughter Board

The daughter board typical receive operation is shown in the Figure 1.5. The RF signal is received through the antenna and then filtered to remove the interference. The process may be either band-pass filtering or low-pass filtering. The LNA - low noise amplifier helps to increase the signal strength as against the noise floor. The increased signal strength helps improve the dynamic range over which the signal is digitized by ADC. The mixer following the LNA allows the conversion of RF to IF and quadrature mixing to form I-Q component signals. This IF signal(s) are fed into ADC to form digitized samples and undergo further processing on the mother board before going to the host computer [6].

The daughter boards transmit operation is shown in Figure 1.6. The output of the DAC is analog IF signal (or signals if quadrature samples were input to the DAC) that is fed into the mixer on the daughterboard which converts them into analog RF. In case quadrature samples were fed to the DAC, then the IQ signal streams that are produced from DAC are summed in the mixer to produce a single signal at carrier frequency. The signal is then amplified for transmission over desired distance to the intended receiver. The amplified signal is then filtered 
to remove any interference produced by the non-linearity in the daughterboard or motherboard components. This is the signal that is finally transmitted via the antennas attached to the daughterboard.

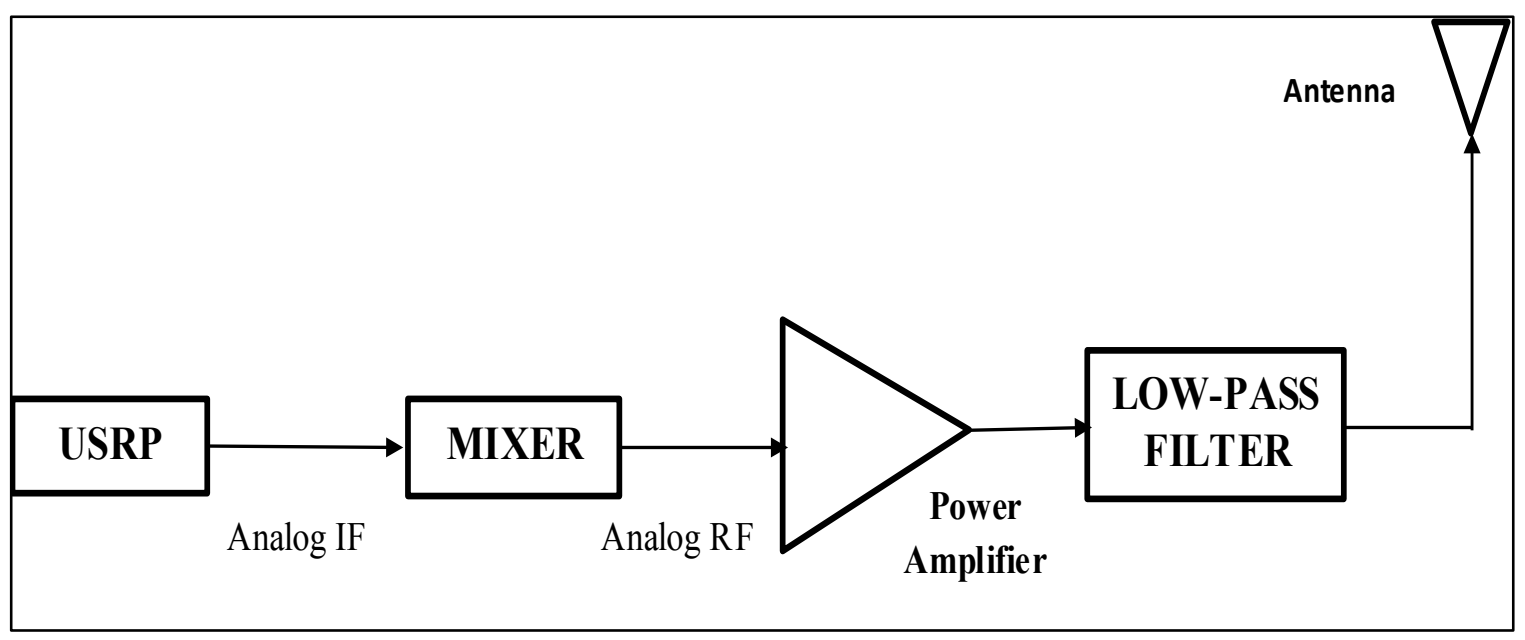

Figure 1.6 Block Diagram representing Transmit Operation of Daughter Board

USRP-X series device (USRP $\mathrm{X}-300 / \mathrm{X}-310$ ) allows up to 4 simultaneous radio transmission i.e., 2 receivers and 2 transmitters with a connection of 2 daughter boards at same time but many of other USRP models allow only 1 daughter board interface. USRPN210 model used for the experimentation also allows only 1 daughter board connection at a time. There are different types of daughter boards that can be used in conjunction with USRPN210 for TX and Rx operations. SBX board allows operations from $400 \mathrm{MHz}$ to $4.4 \mathrm{GHz}$. This is a transceiver board allowing transmit and receive operations simultaneously too i.e. full duplex operations with a maximum operating bandwidth of $40 \mathrm{MHz}$. This board is usually used to emulate GPS reception, Radar, Wi-Fi and WiMAX operations. The bandwidth utilization largely depends 
upon the USRP main device the daughter board is paired with and the resolution (bits per sec) of the data transferred. WBX board allows operations from DC to $2.2 \mathrm{GHz}$. This is again a transceiver board allowing full duplex operations along with simple transmit and receive. The bandwidth is also $40 \mathrm{MHz}$ and this board is used for multiple applications like TV broadcast reception, GPS reception, public safety transceiver kit, OPEN BTS -GSM base station and amateur radio operations. WBX is a MIMO capable daughter board [3] [9].

\subsection{Literature and Documentation - USRP and GNU Radio}

It is known that GNU Radio can be effectively combined with hardware peripherals like USRP(s) developed by Ettus Research to develop Software defined radios. Numerous studies have also demonstrated the same successfully [10]. As GNU Radio is an open source platform for software radio development, it also provides flexibility in the types of applications developed and the feasibility to emulate wide-range of practical implementations like radars, sonar, weather surveillance, sensor networks etc., [4].

Internet has a lot of open-source information on GNU radio and USRP, however they are not very well documented and archived to enable proper learning. They are usually personal blogs, or community forums that are a pool of information from multiple authors and hence may be inconsistent. Some of the main sources of information on GNU Radio and USRPs are the official Ettus Research and the GNU Radio websites. The schematics of the hardware with the datasheets can also be found there [4]. Vendor company or third party utilities have the schematics on their respective websites. There is limited information available on the internal architectural details of the USRP devices and/or their basic implementation details. Many of these are not-up-to-date and refer internal structure of the initial USRP1, due to rapid progress in 
development of Software defined radios and field of wireless communications in general. GNU Radio and Ettus Research support discussion forums that enables users to ask specific questions and learn. However, many a times they address queries very specific to the application the user wishes to develop; they do not address general doubts or provide clarification regarding any of the basics. For example, the in depth documentation on pre-defined inbuilt programs like UHD_SIGGGEN.py, UHD_FFT.py cannot be found to answer questions like how do we know the actual received power or the transmitted power? Even the links on GNU Radio address the functionality of the block on an overall level but do not give a detailed review of its functionality with examples for illustration and easier understanding.

Thus, the documentation available provides just a good starting point for basic understanding of USRP and GNU Radio functioning. They do not provide any clear details regarding the performance or characteristics like the maximum power transmitted through the RF1 (TX) port when using UHD_SIGGEN.py program or the power that can be expected for the given set of input parameters or the normalization factor and scale used in UHD_FFT.grc for plotting the received spectrum from RF2 (RX) port. These details like the behavior and performance ability/limitations are very much necessary in order to leverage the full potential of USRP based test beds and other experimental applications.

\subsection{USRP based Test Beds}

In the recent past, several test beds using USRP devices have been setup for enabling research on cutting edge wireless technology. One of such extensive test beds is housed on our own Virginia tech Campus in Kelly Hall under the supervision of ICTAS (Institute for Critical technology and Applied Sciences Building), generally known as the CORNET for Cognitive 
Radio Network Test bed. This has created a need to understand the basic performance and characteristics of USRP devices. It is very important to build reliable wireless systems to facilitate effective communication and understanding the behavior of the devices used well in turn can facilitate this [11].

\subsubsection{CORNET Test Bed}

The CORNET - Cognitive Radio Network Test bed is a set of 48 nodes housed in Kelly Hall, ICTAS - Institute for Critical Technology and Applied Sciences Building in Blacksburg campus of Virginia Tech across 4 floors as shown in Figure 1.7 [12]. This test bed is one of its

kinds especially dedicated for purpose of research-development and education on multifaceted cognitive radios and its applications. It is an open-source platform, which provides a flexible RF front ends, re-configurable open-source software resources along with FCC license for experimentation. Similar test-bed can be found [13] that also enables rapid prototyping and algorithms' testing of the cognitive radios. At Virginia Tech's CORNET, the nodes are basically USRP2 devices with a WBX RF daughter board with an operational range of DC to $2.2 \mathrm{GHz}$. The USRP2 along with the open-source GNU Radio software allows for very flexible cognitive radio algorithms.

The USRP2 has the basic specifications as seen in Table 1.1. The USRP2 uses gigabit Ethernet as the interface with an instantaneous RF bandwidth of $25 \mathrm{MHz} @ 16$ bits. 


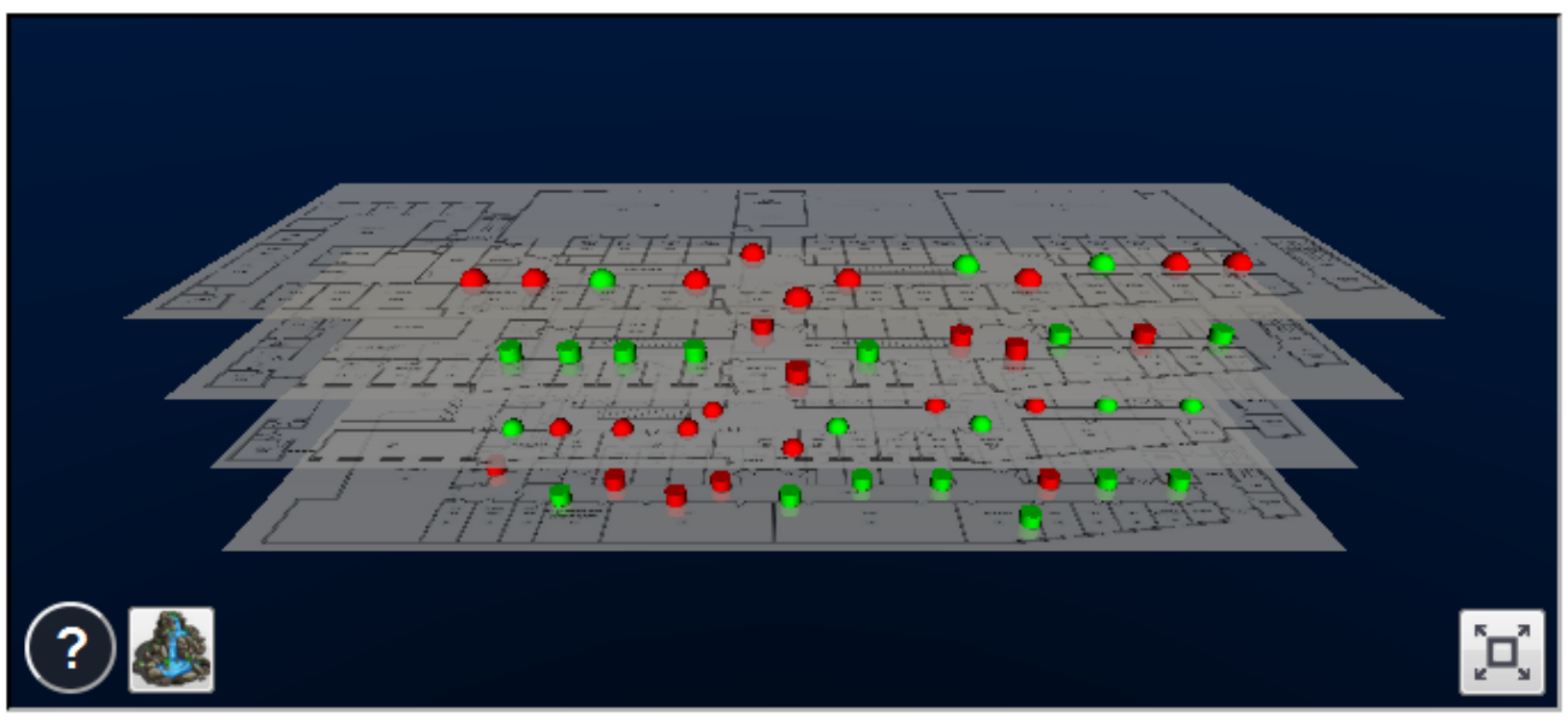

Figure 1.7 CORNET Test-bed (3-D image of the nodes across 4 floors)

Virginia Tech having a special focus on wireless communications and its advancement is on its way to building up another extensive test bed for research and development purposes. This test bed has approximately 15 nodes deployed across the entire campus in Blacksburg in different buildings. Hence they are aptly called the "Outdoor-CORNET or the O-CORNET". These nodes are however USRPN210 devices with SBX transceiver RF boards which allow operations from $400 \mathrm{MHz}$ to $4.4 \mathrm{GHz}$.

Some of the applications that have been developed through CORNET are: CORNET 3D: Spectrum Visualization in 3D [14]; MICRONET: Micro Cognitive Radio test bed; Open Source LTE Project: LTE DL signal emulation and analysis; Spectrum Sensing and Spectrum Access [12]. Apart from the applications mentioned above a number of research projects are currently underway through CORNET/O-CORNET like Position location, Cognitive engines and genetic algorithm development, electronic warfare /Cognitive Jamming etc., [12]. 
Although the CORNET test bed has been successful in its main aim of research and development of Software defined Radios and its applications, one of the major limiting factors of the CORNET and O-CORNET are their distance to the real-world values and accuracy. The hardware used in CORNET and O-cornet allows the applications to be developed relative to the software programs' and their implementation and allow evaluation particular to that SDR. They often do not explore or measure the characteristic behavior of the SDR in more general realistic sense. For example a power measure given in the 3D graphic visualization of CORNET is relative to the scale set in a built in program UHD_FFT.py that helps to visualize the received RF spectrum. But it cannot be said for sure, if this power is the correct value of received power in $\mathrm{dB} / \mathrm{dBm} /$ Watts and is close to the expected value. A software program like UHD_FFT.py often normalizes the $\mathrm{Rx}$ power, Rx gain so as to enable coherent and real-time spectrum plot. However it is very difficult to understand the factor of normalization as the USRP houses several analog stages of amplification and filtering before being passed to ADC unit [15]. This may in turn lead to another set of normalized values like BER, EVM, which are based on calculations of the metrics.

Thus, in order to enable the relationship of the CORNET applications and its so-derived metrics to real-world metrics, basic calibration and characterization of the hardware used (USRPN210) was undertaken as the main focus of this thesis.

\subsection{Review and Goals}

The basics concepts learnt in the Software Defined Radio Course at Virginia Tech were one of the foremost driving factors for interest in this area. Further reading of USRP and GNU Radio tutorials and wiki links proved useful in understanding the working of hardware and 
software signal processing utilities and experimenting with them. Previous research work in this area done by Terrence J. Brisebois [6] and Michael Maxwell Hill [4] provided insight into performance and capability measures of this combination.

One of the major drawbacks evident from the literature review seemed to be the lack of information on effective calibration of the USRP devices, creating an inability for the results to be related and understood in terms of real world metrics [15]. This limits their utility for rigorous experimentation. There were many questions that seemed to be unanswered but are very much a requisite in case USRPs are to be used as standard radio front ends for software defined radio systems. Some are: Can USRP be characterized using any standard methods? Is there a significant variation among the USRPs units of the same model and daughter board combination? Is there any variation between different USRP models and daughter board combinations? Is there a possibility to derive standard factors or look up tables for the calibration of each USRP- daughterboard combination? Is the calibration specific to the software utility used or would it be standard across different utilities and different software packages?

Practical experiments like Position location estimation based on RSS would require precise received power measurement so as to be able to characterize the channel correctly, model the path loss accurately and thus reduce the error in position estimation. Fingerprinting is one such distance-based calibration commonly used in RSS based position location methodology wherein each reference node along with the measure of the received power also communicates its coordinates to the main server periodically. This helps first create a Radio Map for the node environment offline. It also helps track the received power strength as the location of the reference node varies and model the path loss and channel precisely in the real time 
measurement [16]. Real time based calibration suggested in [17] for indoor position location is also based on RSS which tracks the power received at each node and re-estimates the path loss exponent and characteristic constant each time (for each iteration) and uses it for the next set of measurements.

\subsection{Thesis Outline}

The main aim of this thesis was to obtain a relation between the readings obtained on a spectrum analyzer utility provided under GRC with the real world metrics like $\mathrm{dBm}$ for power. A software-defined radio was implemented using the USRPN210 with the GNU Radio software. The RF daughter board used for the tests was mainly WBX transceiver boards with a broad frequency range from $\mathrm{DC}$ to $2.2 \mathrm{GHz}$. Characterization tests like the received power stability test, the transmitted power and frequency stability tests were done and the values obtained were studied in order to understand the difference in the value on UHD_FFT.grc graph and the dBm metric value obtained on the Tektronix spectrum analyzer, when the same experiments were conducted with it.

Next the correction factors for various parameters like power, frequency were utilized in an application like position location estimation and indoor multi-floor path loss modeling in order to check if there was any improvement in the estimated values with regard to expected results.

In this thesis, the first chapter gives the background on the key concepts like software defined radio, USRPs, GNU Radio and also expands into topic of daughter-boards along with review of the literature while the second chapter details the main idea behind this thesis and its research methodology. Chapter 3 describes the experimentation methodology by detailing the 
experimental setup, test background and parameters used for the tests while Chapter 4 describes experimentation procedure along with the results where the results obtained are compared with the actual expected results and conclusions are drawn. Chapter 5 concludes by summarizing the overall intent of the thesis along with the outcomes. 


\section{RESEARCH METHODOLOGY}

In order to be able to relate the applications developed through use of USRPs and GNU Radio to real-world metrics, the characteristics of the USRP hardware were studied and analyzed. This lead to development of calibration factors that could be used with the data obtained during any experiments and enabled them to be processed as real-world metrics.

This chapter describes the hardware and the software used specifically for the experimentations. Utilizing existing and commonly used software utilities of GNU Radio like UHD_FFT.py, RX characteristics of the USRP was studied across different units and the variation if any was noted. Similarly the variation on the transmitted side was studied using UHD_SIGGEN.py utility and a standard spectrum Analyzer. The factors thus obtained were used for experiments to locate transmitter position using Received Signal Strength based method and its enhanced version. For the path loss modeling using the multi-floor CORNET, the log-distance model was used.

\subsection{Calibration of USRPs - Hardware and Software}

As the main purpose of this work is to examine how the data obtained through use of USRP and GNU Radio combination can be effectively related to real-world metrics, the investigation was focused on achievable TX and Rx performance with a set of inputs rather than the performance capabilities/limitations with respect to a specific application. The basic hardware requirements were as follows:

1. DUT: The device under test was the USRPN210 with WBX boards. This allowed obtaining the calibration factors for the highest performing USRP hardware available. A set of 10 such 
USRPN210 was used to create a sample set. The specifications of the USRPN210 devices are as seen below and a picture of the USRPN210 device has also been provided [8].

Table 2.1 USRPN210 Specifications, (C) Matt Ettus. Used with permission. See Appendix B. Permission from Matt Ettus

\begin{tabular}{|l|c|}
\hline Specifics & Value \\
\hline Interface & Gigabit Ethernet \\
\hline Instantaneous RF bandwidth & $25 \mathrm{MHz} @ 16$ bits \\
\hline Xilinx Spartan & 3A-DSP, 3400 FPGA, onboard processing \\
\hline ADC & 16-bit, $100 \mathrm{MSps}$ \\
\hline ADC & MSps \\
\hline Frequency Accuracy & with GPSDO reference \\
\hline Storage Configuration & Networked series/stand alone \\
\hline Operation & MIMO with 2 or more systems \\
\hline Multiple system capability & 1 TX / Rx or 1 transceiver \\
\hline Daughter Board Capacity & \\
\hline
\end{tabular}

2. RF Daughter board and Operating Frequencies: Since the WBX Daughter boards were used with the USRPN210(s) for the experiments (which allow operation in DC to $2.2 \mathrm{GHz}$ range [9]) the experiments were performed in the $400 \mathrm{MHz}, 900 \mathrm{MHz}$ and $1.8 \mathrm{GHz}$ so as to enable learning and generation of calibration factors in 3 ranges - low, mid and high frequencies. 


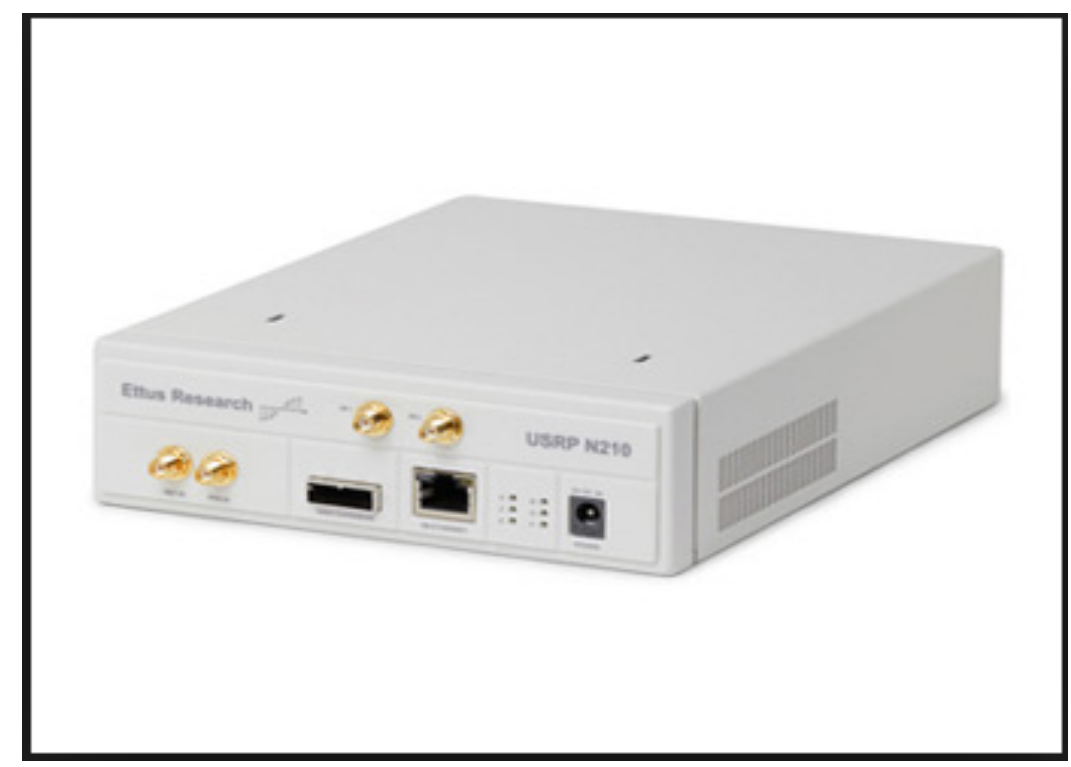

Figure 2.1 USRPN210 Device, (C) Matt Ettus. Used with permission. See Appendix B.

\section{Permission from Matt Ettus}

3. Host Computer: A Linux based system was used with Ubuntu 12.10, 64 bit operating system. A Debian based system is widely used and also supports the different version of GNU Radio software. The processor specifications are Intel Core i7-3840QM @ $2.8 \mathrm{GHz} * 8$.

4. GNU Radio software: GNU radio software downloadable file is available from the ETTUS Research website. They usually provide software releases supported by LINUX distribution system. The GNU Radio Companion version used was 3.6.4.1 with GNU C++ version of 4.4.3 [18].

5. UHD Firmware: This is the "USRP Hardware Driver" that provides driver interface and API for ETTUS Research products. The version installed in the host computer was UHD_003.005 compatible with GNU radio version 3.6.4.1 and Ubuntu 12.10 [19] [20]. 
The Spectrum Analyzer utility provided by GNU Radio is shown in Figure 2.2. This helped to observe the spectrum of the USRP behaving as receiver.

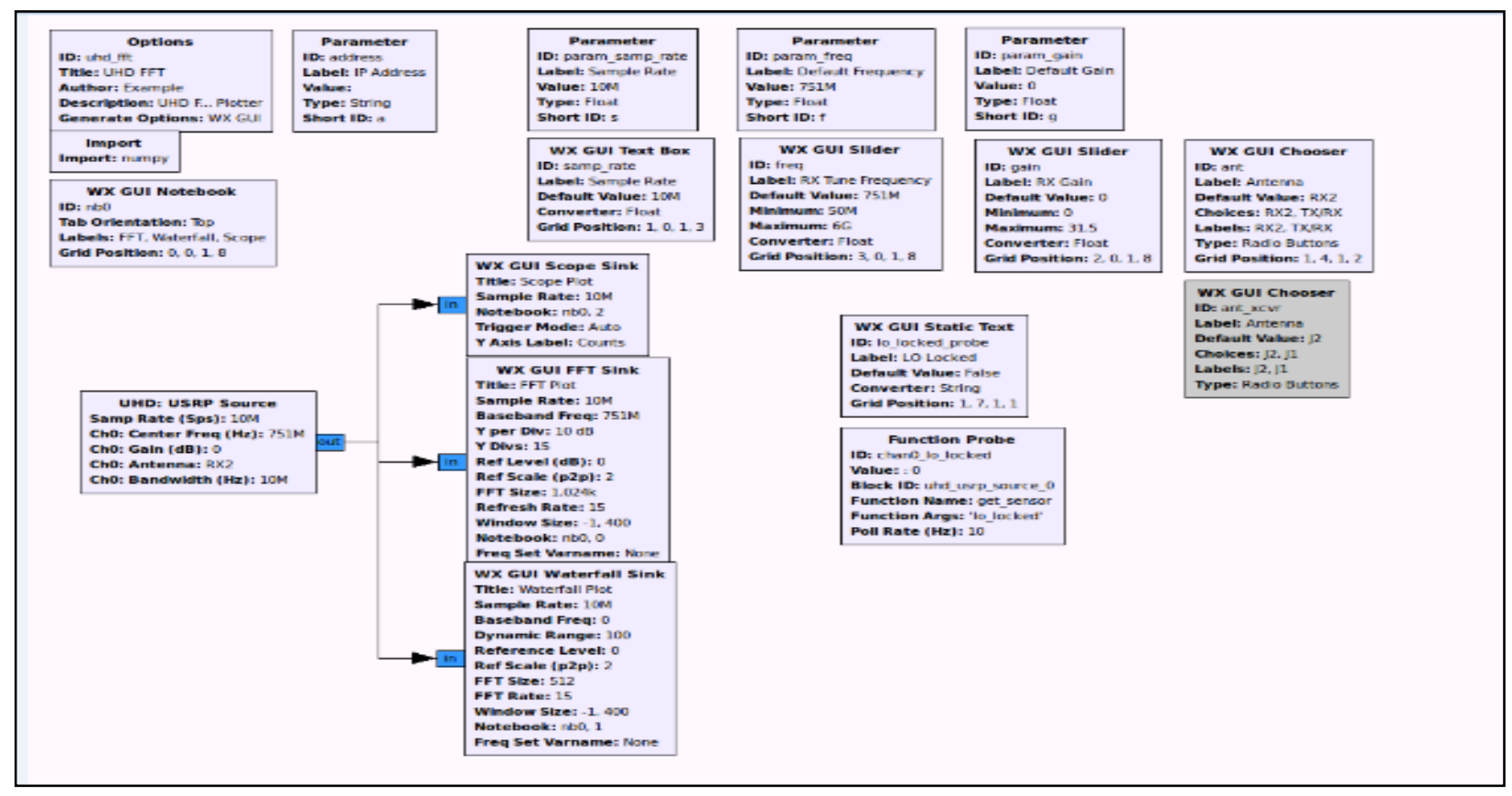

Figure 2.2 Flow Graph emulating Real Time Spectrum Analyzer, (C) Matt Ettus. Used with permission. See Appendix B. Permission from Matt Ettus 


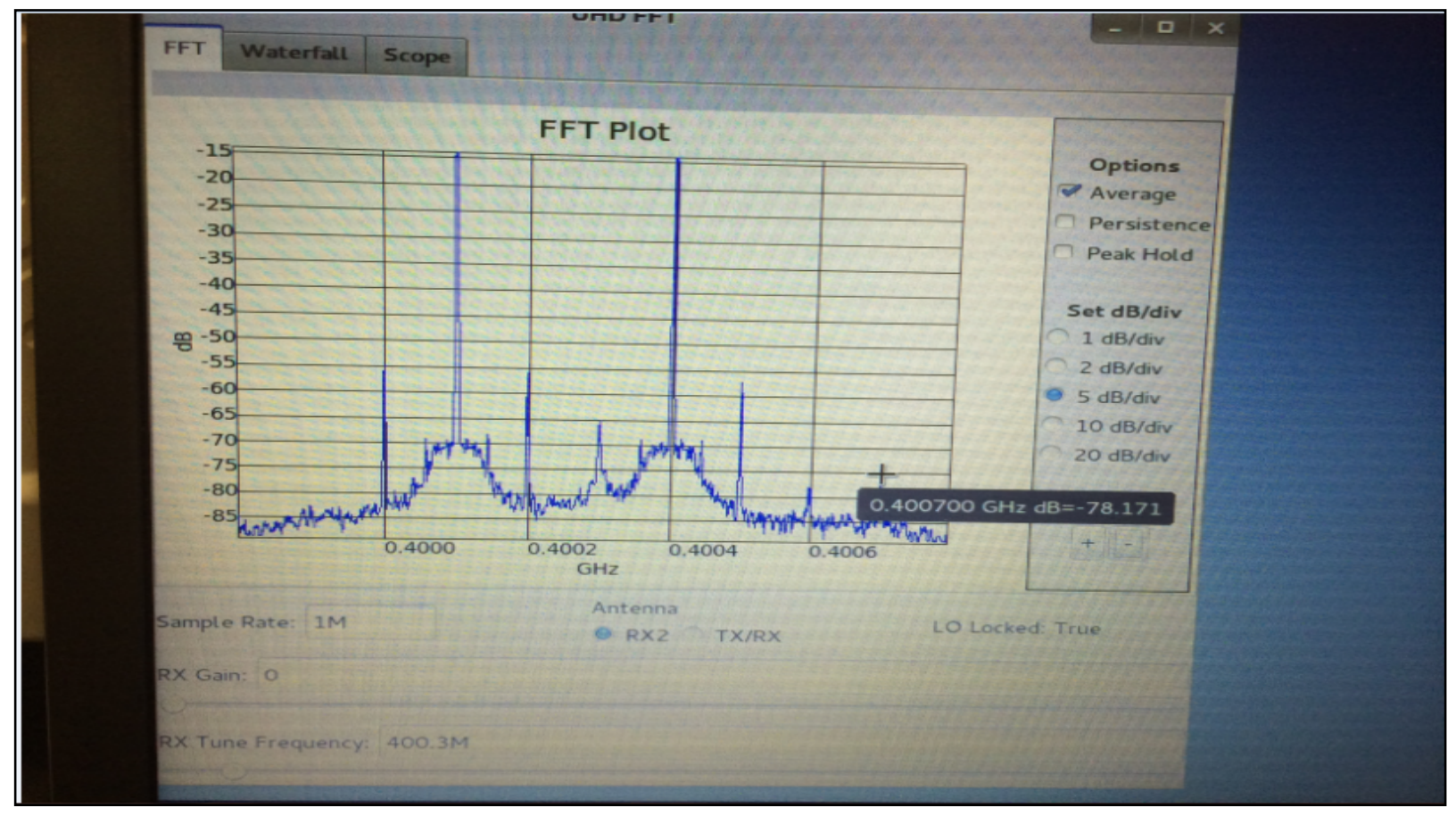

Figure 2.3 Sample Output of Spectrum Analyzer Utility

Since a set 10 similar USRPN210(s) were available and therefore were used as a Sample set, the values obtained per USRP per test could be easily averaged over assuming uniform behavior. But for the purpose of comparisons, the same tests were performed on a USRP2 with WBX board and USRPN210 with SBX board.

Some of the operational limitations with USRPN210s are posed by the host connection bandwidth (connection between has computer and the USRP), antenna functionality range (they may fall below the required SNR performance for a particular application). For any radio application, the transmit power is also an important metric affecting the SNR at the receiver end and thus the detection range. Usually for a USRPN210, as per the specifications, it is preferable 
to keep the maximum transmit power below $+20 \mathrm{dBm}$ at the $\mathrm{TX}$ port (RF1) and maximum receive power at any $\mathrm{Rx}$ port $(\mathrm{RF} 1 / \mathrm{RF} 2)$ to be below $-10 \mathrm{dBm}[\mathbf{2 1}]$.

\subsection{Practical Application of Calibration Factors}

The calibration factors were determined as an average of the values obtained from performing the tests on the 10 USRPN210s under similar test setup and conditions. However the limitation factors mentioned afore would come into play even while performing these experiments and the calibration factors thus obtained need to be verified.

In any practical real-time radio system, the loss affecting the transmitted signal from the time of transmission until the point of reception generally called the Path loss is a major concern. Path Loss Modeling along with the methods to minimizing effective path-loss, increasing the integrity and security of the data signal transmitted, and also reducing the processing on receiver end is a major area of research in wireless communications. With onset of wireless protocols standards like Wi-Fi, Bluetooth, indoor path loss modeling and propagation characterization has also generated a lot of interest. Intra-vehicular propagation modeling and channel characterization using $5 \mathrm{GHz}$ band [22] is a hot research area since technology with mobility is the new age requirement for consumers (E.g. Wi-Fi connectivity in airplanes is common place) and also with the fact that ISM $2.4 \mathrm{GHz}$ band having been utilized to its maximum has now the

researchers finding newer spectrum avenues with lower bandwidth restrictions (E.g. $5 \mathrm{GHz}$ band is not fully explored). In this thesis indoor multi-floor path loss modeling with assuming Logdistance distribution within CORNET has been utilized to check if calibration factors have any impact on the estimated path loss exponent using method suggested in [10] [23] [24] and if the values adhere as per expectations. 
Another application causing much interest in wireless communication is Position Location Estimation. There are different methods of determining the position location of a transmitter such as Position Location using signal Angle of Arrival, time of arrival, Received Signal Strength etc. The Position Location using RSS is one of the simplest methods, with low receiver end processing and also applicable to indoor wireless systems, which allow a good estimate of the unknown sensor and with progressive improvement in algorithmic techniques like fingerprinting, this method has been shown to be very deterministic with low error coefficient.

Here, the CORNET test bed was used to perform the Path Loss experiment across multifloors to determine the path-loss exponent best suited for such signal propagation. The basis of the methodology was the research done on 914 and $2.4 \mathrm{GHz}$ bands previously for indoor propagation [10] [23] [25].

The position location experiment was performed using the RSS methodology. The main amongst these experiments was to show that the calibration factors obtained from the characterization experiments helped to gain closeness to real-world practical values, while the values taken directly from the UHD_FFT.grc GUI plot do not give any meaningful results.

It is important to understand that the multi-floor path loss calculation on the CORNET Test bed and the Position location experiments were conducted indoors. Hence the received power would not be affected as in the typical free space manner and would need to be handled differently. In both the experiments the Received Signal Strength is the important measure for the calculation and the correction factors would be mainly applied to that. 


\subsubsection{Basic Propagation Mechanism}

A radio system generally is said to undergo Free Space propagation when the transmitter and receiver have a line of sight and the distance between the transmitter and receiver antennas is in terms of kilometers. The transmission should also not have any other sources of impairment like scattering, diffraction leading to multiple paths of signal transmission. For e.g. Satellite systems and microwave communication is said to undergo free space propagation. The loss that is seen in such a transmission system is commonly termed as free space path loss and the equation to calculate the theoretical loss under the given set of conditions (distance, TX power, Rx power) is given by the FRIIS path loss equation for unity gain, shown below [10]:

$$
F S P L=\frac{P_{t}}{P_{r}}
$$

where FSPL is the free space path loss, $P_{t}$ is the power transmitted from the transmitter antenna and $P_{r}$ is the power received at the receiver antenna. FSPL can also be expressed logarithmically in $d B$ as seen below [10].

$$
F S P L(d B)=10 \log _{10} P_{t}-10 \log _{10} P_{r}
$$

where $P_{t}$ and $P_{r}$ are the power transmitted and received at the transmitter antenna. The relation between transmit and receive powers can also be expressed as

$$
P_{r}=P_{t} * G_{t} * G_{r} *\left(\frac{\lambda}{4 \pi d}\right)^{2}
$$


where $G_{t}$ and $G_{r}$ represent the transmit and receive antenna gain respectively, while $L$ is the wavelength, $d$ is the transmitter to receiver separation, $P_{t}$ represents the transmitted power and $P_{r}$ represents the receiver power [10].

As per the FRIIS Free space path loss equation, the receiver power is said to decrease as the square of the distance between the transmitter and receiver which is

$$
P_{r} \propto\left(\frac{1}{d}\right)^{2}
$$

where $P_{r}$ is the receiver antenna power and $d$ is the distance between the transmitter and receiver. Thus the received power decays as $20 \mathrm{~dB} / \mathrm{decade}$.

The three basic modes of wave propagation are reflection, diffraction and scattering. Reflection is the phenomenon by which the electromagnetic wave impinges on an object but does not undergo a change on its medium of propagation. Diffraction is the phenomenon by which the electromagnetic wave impinges on an object then undergoes a change in its medium of propagation. This usually occurs when there are objects with sharp edges in the propagation path. Scattering is the phenomenon by which the electromagnetic wave is split into a number of subwavelets, after impinging on the object. This usually occurs when the path contains objects that are very small in dimension as compared to the wavelength and the number of obstacles per unit volume if large. The path loss equation in such scenarios is more conveniently expressed in terms of a reference distance $d_{0}[\mathbf{2 2}]$. 


$$
P_{r}(d)=P_{t} * K *\left(\frac{d_{0}}{d}\right)^{2}
$$

where $K$ is a characteristic constant that depends on the antennas characteristics and $P_{r}(d)$ is the power received at a distance of $d$ from the transmitter; $P_{t}$ is the transmitter power and $d_{0}$ is the reference distance usually set as less than or equal to one meter for indoors and between 100 meter and one kilometer for outdoors. A simpler model that can be used (tradeoff analysis) for this is detailed as

$$
P_{r}=P_{t} * K *\left(\frac{d_{0}}{d}\right)^{\gamma}
$$

where $\gamma$ represents free space with a value of 2. The value of $\gamma$ for urban outdoor area ranges between 2.7 to 3.5 while $\gamma$ for sub-urban outdoor ranges between 3 and 5 and $\gamma(L O S)$ for indoor environment ranges between 1.6 and 1.8 [22].

The position location experiments in our case were conducted within a large room, with many types of obstacles around. Thus though there was a line of sight propagation, the large number of obstructions around do not allow easy categorization of the extra losses undergone by the signal. Thus the experimental path loss may differ marginally from the theoretical expected. Another important factor to consider while performing Path loss or Position Location calculation is the Fading. There are different types of fading models that have been generated based on the different scenarios studied. The most basic ones are Ricean, Rayleigh, Hata, Okamura and Weibull [24] [26]. 
The CORNET Test bed for path loss determination and test set up for position location using RSS, do not conform to any of the models stated above perfectly. This will be more extensively analyzed in Chapter 3 and 4.

\subsubsection{Multi Floor Path Loss using CORNET}

Many indoor path loss and location information prediction models have been proposed through previous research [27] [23]. These models enable the understanding of how propagation characteristics are affected by the obstacles and surrounding environment especially in closed building scenarios where the transmitter and receiver might not be in the same floor too. The models have been shown to provide more accurate results than the generic free space model. Based primarily on the distance dependent log-distance model that implies that the mean path loss increases exponentially with distance, the relationship is formulated as [23]:

$$
P L(d) \propto\left(\frac{d}{d_{0}}\right)^{n}
$$

where $P L(d)$ is the path loss at a distance $d, n$ is path loss exponent, $d_{0}$ is the reference distance and $\mathrm{d}$ is the transmitter receiver separation distance. On the logarithmic scale, the formula is given as $[\mathbf{2 3}]$ :

$$
P L(d B)=P L\left(d_{0}\right)+10 n \log \left(\frac{d}{d_{0}}\right)
$$

where $P L\left(d_{0}\right)$ is the path loss at a reference distance (generally taken as one meter) under free space propagation. $n$ is the path loss exponent, which is a function of the number of floor 
between the transmitter and receiver, building type and the building wing. Re-arranging the above equation $n$ is given as:

$$
n=\frac{P L(d B)-P L\left(d_{0}\right)}{10 \log _{10}\left(\frac{d}{d_{0}}\right)}
$$

In our experiment to verify the calibration factor, the CORNET nodes are used to determine the path loss between a transmitting node on first floor and all the other nodes. This path loss value is then used in the equation 2.9 to determine the path loss exponent through method of best fit with least deviation from the measured path loss.

The average of all the path loss exponents for one floor is determined to get the mean path loss exponent per floor. The deviation from this mean path loss exponent value of each individual exponent measure can also be calculated.

Another model used for indoor propagation path loss modeling is the ITU model [24] [28]. This is based primarily on the free space path loss model equation 2.2 .

$$
L_{t}(d B)=20 \log f+N \log d-27.54
$$

where $f$ is frequency in $\mathrm{MHz}$ and $d$ is the distance in meters. Here $N$ is the Power loss coefficient given by path loss exponent $n$ value multiplied by factor 10 . In this ITU model, path 
loss exponent $n$ or the power loss coefficient both are dependent on the number of floor between the transmitter and receiver. The loss $L_{t}$ is inclusive of the floor attenuation [10] [23].

However the formula can be rearranged to get the Floor Attenuation Factor $(F A F)$ as:

$$
L_{f}(d B)=20 \log f+10 n_{\text {same floor }} \log d+F A F-28
$$

For calculating the Floor Attenuation Factor $(F A F)$ which is also dependent on the number of floor between the transmitter and receiver nodes; the path loss exponent $n$ used is the mean value found using equation 2.9 for the same number of floors i.e. if we need to find the find the $F A F$ for three floors between transmitter and receiver, then $n$ used is the mean exponent value found for three floors using equation $2.9[\mathbf{2 3}]$.

\subsubsection{Position Location using Received Signal Strength}

This is a method used to determine the position location of a target device based on the power received between two nodes and depending upon a specific path loss model. The path loss can be said to have a constant and a variable attenuation factor. The constant factor is dependent on the chosen path loss model, while the variable factor is subjective to the environmental conditions resulting in shadowing, multi path etc., [24].

At least 3 receiver (reference) nodes along with transmitter (target) nodes are required to estimate the position using RSS. The transmitter node continuously transmits the signals to the receiver nodes. The power received by each of the receiver node is measured and processed in 
order to determine the location of the target (transmitter here) node. As mentioned afore, the free space propagation model gives the power received as:

$$
P_{r}=\frac{P_{t} G_{t} G_{r \lambda 2}}{(4 \pi d)^{2}}
$$

where $P_{r}$ is the received power at the nodes; $P_{t}$ is the transmitted power from target node; $G_{t}$ and $G_{r}$ are the respective antenna gains.dis the distance of each node form the transmitter which can be re arranged as:

$$
P_{r} \propto\left(\frac{1}{d}\right)^{2}
$$

This implies that power received varies with distance ' $d$ '. The main goal is to estimate the coordinates of the target node, having known the position coordinates of the reference nodes. For this the multi-lateration technique is used whereby each reference node is seen to be the center of a circle. The target node is said to be on the circumference of each of these circles. Thus the distance between the target and each of the reference nodes becomes the radii of their respective circles. The intersection of these 3 radius measures is the location of the target node, which is obtained by method of least squares [29]. Hence, if the distances of each of the reference nodes is given as [24]: 


$$
\begin{aligned}
& D 1=d_{0}\left(\frac{P_{0}}{p r_{1}}\right)^{1 / n} \\
& D 2=d_{0}\left(\frac{P_{0}}{p r_{2}}\right)^{1 / n} \\
& D 3=d_{0}\left(\frac{P_{0}}{p r_{3}}\right)^{1 / n}
\end{aligned}
$$

Using the maximum likelihood estimation method, the coordinates $\left(x_{t}, y_{t}\right)$ are determined. If $\left(x_{1}, y_{1}\right)\left(x_{2}, y_{2}\right)$ and $\left(x_{3}, y_{3}\right)$ are the coordinates of the reference nodes, then their respective distance from target node can be given as:

$$
\begin{aligned}
& \left(x_{1}-x_{t}\right)^{2}+\left(y_{1}-y_{t}\right)^{2}=\left(d_{1}\right)^{2} \\
& \left(x_{2}-x_{t}\right)^{2}+\left(y_{2}-y_{t}\right)^{2}=\left(d_{2}\right)^{2} \\
& \left(x_{3}-x_{t}\right)^{2}+\left(y_{3}-y_{t}\right)^{2}=\left(d_{3}\right)^{2}
\end{aligned}
$$

Manipulating to shift the baseline node,

$$
\begin{aligned}
& \left(x_{1}\right)^{2}-\left(x_{3}\right)^{2}-2\left(x_{1}-x_{3}\right) x_{t}+\left(y_{1}\right)^{2}-\left(y_{3}\right)^{2}-2\left(y_{1}-y_{3}\right) y_{t}=\left(d_{1}\right)^{2}-\left(d_{3}\right)^{2} \\
& \left(x_{2}\right)^{2}-\left(x_{3}\right)^{2}-2\left(x_{2}-x_{3}\right) x_{t}+\left(y_{2}\right)^{2}-\left(y_{3}\right)^{2}-2\left(y_{2}-y_{3}\right) y_{t}=\left(d_{2}\right)^{2}-\left(d_{3}\right)^{2}
\end{aligned}
$$

In a linear manner, the above equations can be represented as: 


$$
A X=B
$$

where $A=\left[2\left(x_{1}-x_{3}\right), 2\left(y_{1}-y_{3}\right) ; 2\left(x_{2}-x_{3}\right), 2\left(y_{2}-y_{3}\right)\right], \quad B=\left[x_{1}{ }^{2}-x_{3}{ }^{2}+y_{1}{ }^{2}-y_{3}{ }^{2}+\right.$ $\left.d_{3}{ }^{2}-d_{1}{ }^{2} ; x_{2}{ }^{2}-x_{3}{ }^{2}+y_{2}{ }^{2}-y_{3}{ }^{2}+d_{2}{ }^{2}-d_{1}{ }^{2}\right]$ and $X=\left[x_{t} ; y_{t}\right]$

Finally the solution is obtained as [24]:

$$
X=\left(A^{\mathrm{T}} A\right)^{-1} A^{\mathrm{T}} B
$$

The solution thus obtained from equation 2.23 is the estimate of coordinates of the target node. There are several advantage of this method wherein high accuracy is achieved in cases of LOS and short-range environments. RSS is just the simple received power so no upgrade to hardware is necessary to use this method or even time synchronization (as in Time of Arrival method). In case of finger printing incorporated RSS method, software upgrade might be needed. Multipath, NLOS environments severely affect the accuracy of this method along with the drawback of requiring a minimum of 3 receiver nodes.

In our experiment, a standardized signal transmitter was used as target node and 3 receiver USRPs were used as reference nodes. The received signal strength measured on the UHD_FFT.grc GUI and the calculations conducted with and without correction factor application. This helps determine the effectiveness and need for calibration. The Root mean squared value obtained enables quantitative comparison. 
Another localization algorithm was implemented in order to remove the drawbacks of simple RSS based method. Here the difference between the powers received between two of the reference nodes was considered. Hence this was called as the COMBINED DIFFERENTIAL RSS Method / CDRSS algorithm [30]. The differential power enabled calculation of the individual node weights - which helped in approximating the closer node with higher weight value and thus better position estimation. Again the difference was w.r.t to one of the nodes. This algorithm was given mathematically as:

$$
\begin{aligned}
& X=R S S 1-R S S 3 \\
& Y=R S S 2-R S S 3 \\
& Z=R S S 2-R S S 3 \\
& Q 1=\frac{Y}{X} \\
& Q 2=\frac{Y}{X} \\
& Q 3=\frac{Y}{X}
\end{aligned}
$$

Now solving the following 2 equations, the individual weights can be obtained.

$$
\begin{aligned}
& Q 1: Q 2: Q 3=W 1: W 2: W 3 \\
& \& \\
& W 1+W 2+W 3=1
\end{aligned}
$$


These values then allow the estimation of the transmitter coordinates as:

$$
\begin{aligned}
& \text { x_est }=\frac{W 1 * \mathrm{x}_{1}+W 1 * x_{2}+W 1 * x_{3}}{W 1+W 2+W 3} \\
& \mathrm{y}_{-} \mathrm{est}=\frac{W 1 * \mathrm{y}_{1}+W 1 * y_{2}+W 1 * y_{3}}{W 1+W 2+W 3}
\end{aligned}
$$

This combined differential algorithm is supposed to provide more accurate results than simple RSS based position location.

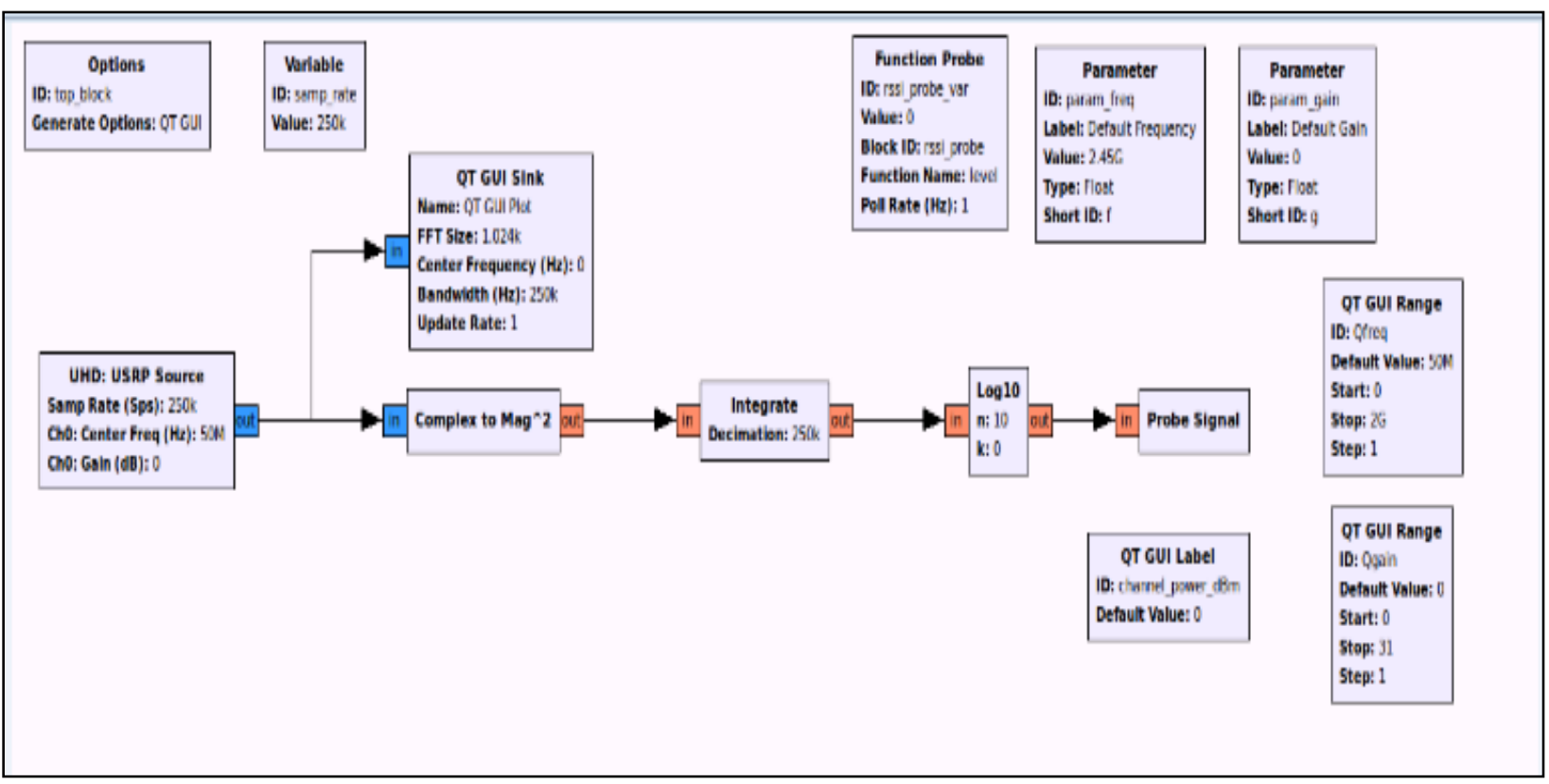

Figure 2.4 Channel Power Estimation Flow Graph for Position Location Experiments

In order to compare the effectiveness of the spectrum analyzer utility itself in giving good power measurements, a new flow graph was also developed to obtain the channel power directly. This was done using the QT Sink block instead of the WX GUI FFT Sink block in GRC and at 
same time, taking the magnitude of the signal power received (RF2) RX port via the Ethernet cable in the software to produce the actual power from ADC. This flow graph was seen to be very user friendly and more useful as the channel power was available directly as a measure in $\mathrm{dBm}$.

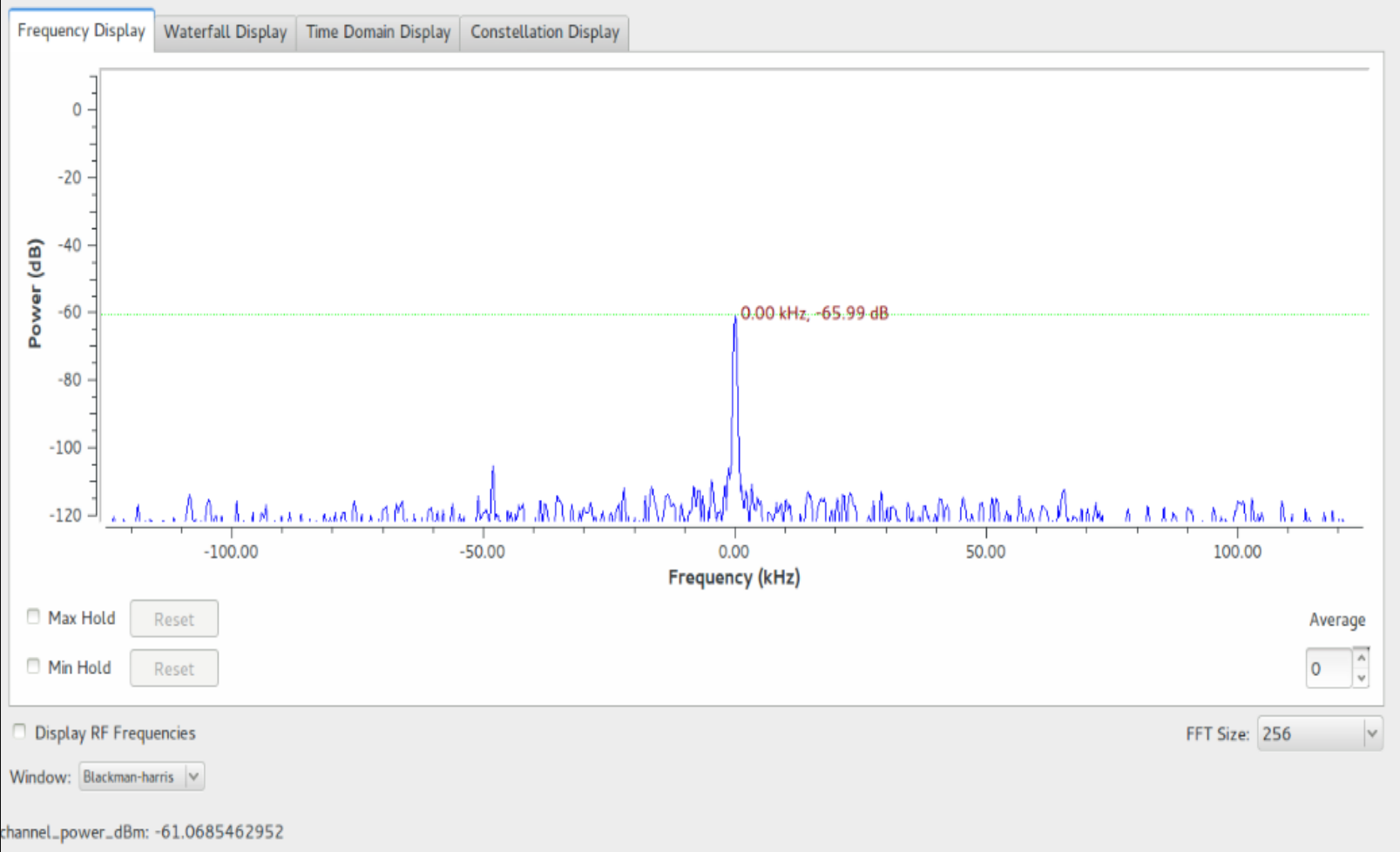

Figure 2.5 Sample Output of Channel Power Estimation Flow Graph

However since the utility changed, the calibration factors estimated w.r.t to UHD_FFT.grc are invalid for this flow graph. This new flow graph based using QT sink itself requires a calibration factor to estimate the correct power specific to the hardware device. Performing test similar to ones performed for UHD_FFT.grc, the calibration factors was found to be around $-62.2 \mathrm{~dB}$ at $400 \mathrm{MHz},-58.8 \mathrm{~dB}$ at $900 \mathrm{MHz}$ and $-52.5 \mathrm{~dB}$ at $1800 \mathrm{MHz}$ for 
USRPN210 device This calibration w.r.t USRP2 and USRPN210 (SBX board) was also determined for comparison. The position location experiment was repeated with this new flow graph using USRPN210 devices and the power received at the 3 reference nodes was noted (for different reference node positions). Both the algorithms (Simple RSS and CDRSS) were tried with and without these new correction factors for USRPN210 device [30]. 


\section{EXPERIMENTAL SETUP AND METHODOLOGY}

This chapter mainly explains about the laboratory setup for the various tests conducted and the different types of equipment used. The basis for each test is also provided along with the justification for the methodology followed for conducting the test. Wherever applicable, standard or previously developed methodology has been adopted (E.g. IIP3 Test [6]). Results have also been discussed briefly.

\subsection{Test Setup}

A system block diagram of the experimental setup for receiver side tests is shown in Figure 3.1 below along with photo of the laboratory setup in Figure 3.2.

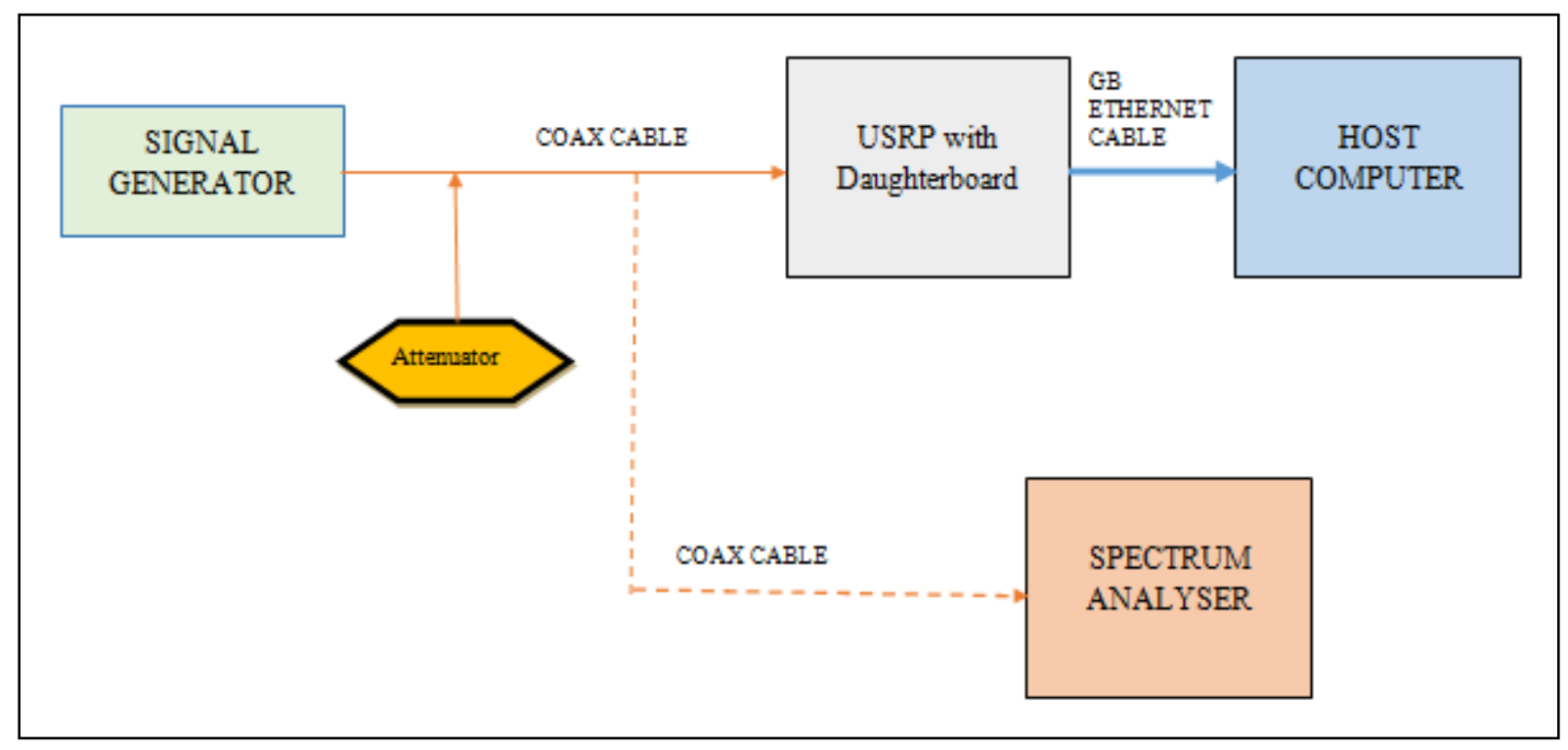

Figure 3.1 Block Diagram of Basic Experimental Setup for RX Tests 
The main devices consist of the spectrum analyzer for output signal measurement and signal generator as a standard signal source. The host system connects to USRP and enables it to either perform transmit or receive operations by executing the in-built application programs like UHD_SIGGEN.py, UHD_FFT.grc or the custom flow-graphs designed using GNU radio

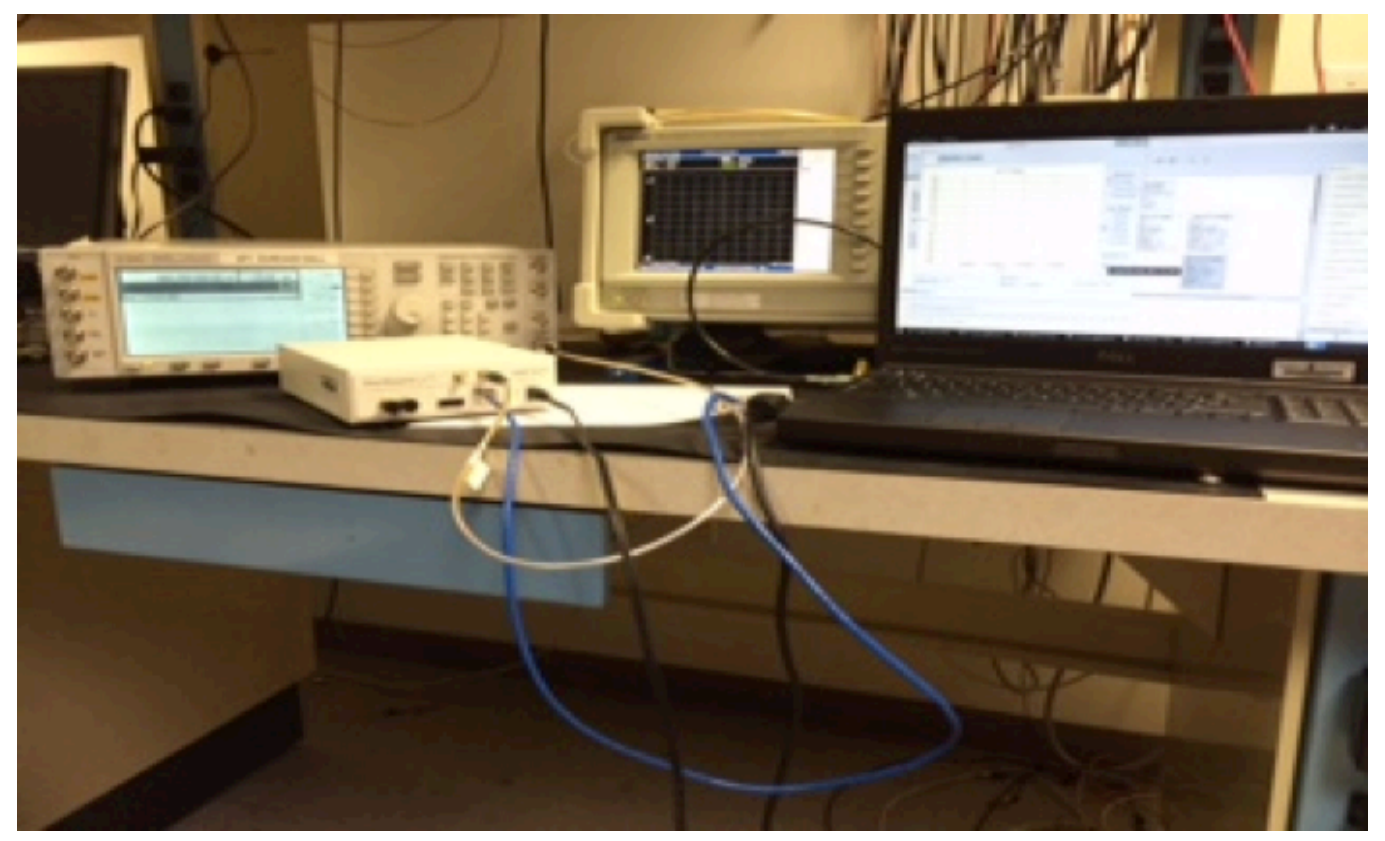

Figure 3.2 Laboratory Setup for RX Characterization

The measurements output spectrum metrics like power and frequency were recorded using the Tektronix RSA 3408A real-time digital spectrum analyzer (DC $\sim 8 \mathrm{GHz}$ ). Mainly used during transmitter characterization, for transmitted frequency stability and amplitude stability test measurements, the device was set on real-time sample and hold so as to be able to measure the average value over acquisition range of 51.2 seconds, with resolution Bandwidth (RBW) of 1 
$\mathrm{MHz}$ and NBW of $2.505 \mathrm{~Hz}$, while for the power measurements since only the peak value was required, the RBW was again set $1 \mathrm{MHz}$ but the mode was on real-time spectrum analyzer mode. The Agilent E4438C ESG vector signal generator $(250 \mathrm{kHz} \sim 6 \mathrm{GHz})$ was the standard input signal source for testing the received power on UHD_FFT.py plot for receiver characterization tests such as the $1-\mathrm{dB}$ compression point test. This also helped provide standard input to calibrate the losses of wires and external attenuators used at different frequencies. For the IIP3 test, another signal generator was needed and hence HP $8648 \mathrm{D}(9 \mathrm{kHz} \sim 4000 \mathrm{MHz})$ was used as the second source of input signal for this test alone. This signal generator was also used for position location estimation. A HP power meter was used for transmitter characterization along with spectrum analyzer.

\subsection{Test Methodology}

The RF characteristics of the USRPN210 were tested with the WBX boards. The total number of USRP devices over which the most of tests was conducted is 10 . The other signal processing equipment used apart from the USRPs were the Spectrum Analyzer and Signal Generator. The same tests were also conducted on a sample USRPN210 device with SBX board and a USRP2 device with WBX board and the experimentation results were compared. These direct comparisons would allow a better understanding of the calibration metrics as applicable to different daughter boards and practical applications. 


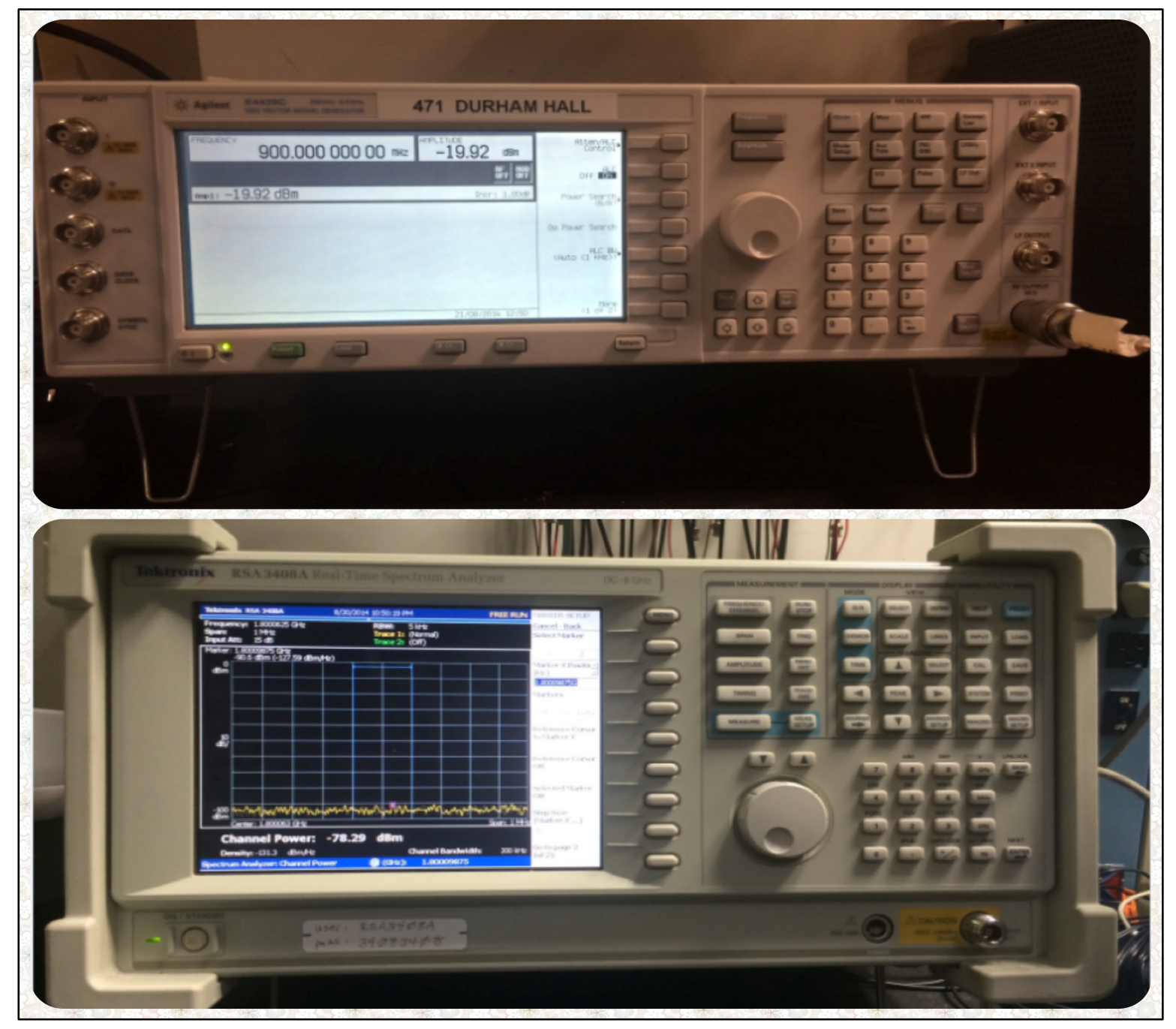

Figure 3.3 Standard I/O Equipment used for the Experiments

Most of the tests were performed at $400 \mathrm{MHz}, 900 \mathrm{MHz}$ and $1800 \mathrm{MHz}$ to represent typical low, mid and high frequency levels of operations of a USRP with RF daughterboard. The tests conducted were a comprehensive set of experiments, which helped in equally evaluating the TX and RX side capabilities of the USRP. 


\subsubsection{The 1-dB Compression Point Test}

Usually a power amplifier's linearity, efficiency and quality can be judged well by two key measurements which are $1 \mathrm{~dB}$ Compression point (1-dBCP): and The third-order Input Intercept point (IIP3).

Amplifier's linearity is very important in any wireless applications especially in today's world, where broadband modulation schemes like WCDMA (Wideband Code Division Multiple Access), HSPA (High Speed Packet Access) and OFDMA (Orthogonal Frequency Division Multiple Access) are common place. Higher power output and efficiency with low heat and power dissipation is vital for applications like mobile handsets, tablets etc., [31].

The 1-dB compression point of a device is a measure of its linear range. The output of the device driven with a good input signal first increases as its input signal increase. However at a point the output signal will not increase proportional to the input signal increase. At this stage the device output is said to start compressing and the output actually starts to decrease with increase in input signal power. The point when the decrease in output signal gain equals $1 \mathrm{~dB}$ with increase in input signal is called the $1 \mathrm{~dB}$ compression point, generally denoted as 1-dBC or 1$\mathrm{dBCP}[\mathbf{3 1}][32]$.

This point has a major significance in the behavior of the device and the expected output. As long as the device is operated below the $1-\mathrm{dBC}$ point, the output of the device would be approximately proportional to the input factoring in the external gain and attenuation applied. However, when the 1-dBC point is passed, the output signal would be distorted and further increase in input would lead to spurious signals or Inter-modulation products. In general, spurious signals would result in interference of the transmitted signal and hence the device is 
best operated well below the $1 \mathrm{~dB}$ compression point. Thus the knowledge of this metric greatly helps in avoiding any undesired/unexpected behavior from a device. The 1-dBC point is generally seen to be $10 \mathrm{~dB}$ below the $3^{\text {rd }}$ order intercept point of the device [31] [32].

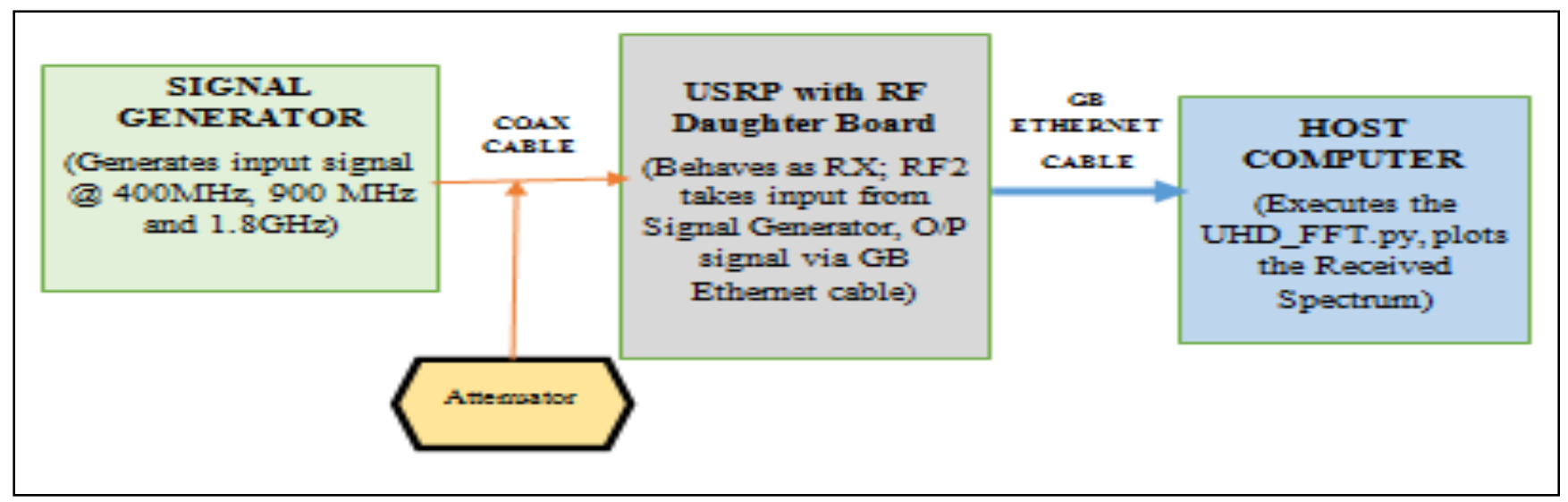

Figure 3.4 Block Diagram of 1-dB Compression Point Test

The complete test procedure is described in Chapter 4 along with the Test results. The basic block diagram of the hardware setup is as shown in Figure 3.4. The output signal power is traced from the plot generated when the UHD_FFT.grc program is executed (an inbuilt program of GNU radio used to plot the spectrum of the output from the USRP device). The USRP device was connected with the host computer via the gigabit Ethernet cable with its RF2 (RX) port connected to the output of signal generator and then the UHD_FFT.grc program was executed. On tuning to the operating frequency (the frequency of the input signal from signal generator) the RF spectrum of signal received by USRPN210 could be seen. The power level from the signal generator was noted. The $1 \mathrm{~dB}$ Compression point test was also conducted at 3 frequencies for understanding the dependence of this point on the frequency. 
However as the input on the receive path to USRP should not exceed $-10 \mathrm{dBm}$, the test was conducted only until this point. The variation of output power Pout measured on UHD_FFT against the input power from signal generator was linear until this point $\left(\mathrm{P}_{\mathrm{in}}=-10 \mathrm{dBm}\right)$ for all the devices. Hence it could be concluded that the USRPN210 with WBX boards do not undergo compression until the maximum recommended input power and hence would show linear characteristics if not exceed this range.

On plotting the difference between the Pin from signal generator and the $\mathrm{P}_{\text {out }}$ measured from UHD_FFT.grc graph, namely the Gain factor, a variation was seen. This was similar for all the 10 USRPN210 devices, which were tested and was different for different frequency of operation. Hence this value was averaged to obtain the mean gain over 10 devices per frequency. This was used as the correction factor for the later experiments. The results across 3 types of USRPs are summarized below in table 3.1 across the frequencies of $400 \mathrm{MHz}, 900 \mathrm{MHz}$ and 1.8 GHz.

Table 3.1 Correction Factors for different USRP Devices across different Frequencies

\begin{tabular}{|c|c|c|c|}
\hline \multirow{2}{*}{ USRP Type } & \multicolumn{3}{|c|}{$\begin{array}{c}\text { Average Received Power offset b/w UHD_FFT } \\
\text { and Spectrum Analyzer }\end{array}$} \\
\cline { 2 - 4 } & $\mathbf{4 0 0} \mathbf{~ M H z}$ & $900 \mathrm{MHz}$ & $1800 \mathrm{MHz}$ \\
\hline & & & 23.57 \\
\hline USRPN210 + WBX board & 35.54 & 31.24 & 20.54 \\
\hline & & & 24.43 \\
\hline USRPN210 + SBX board & 32.19 & & 26.67 \\
\hline & & 32.04 & \\
\hline USRP2 + WBX Board & 32.90 & &
\end{tabular}




\subsubsection{The IIP3 Test}

This is the test performed to find the $3^{\text {rd }}$ order intercept point as a characteristic of the RX operation of the USRP. Two independent fundamental tones (say at F1 and F2 frequencies) separated in few $\mathrm{KHz}$ would generate nth order signals at sum and difference frequency values of the fundamental tones when the amplitude is increased beyond a certain level. These two new signals that are visible at (n-1) F1 $\pm F 2$ frequency point and (n-1) F2 $\pm F 1$ frequency points are the nth order inter-modulation products. In case of $3^{\text {rd }}$ order inter modulation products the two signals occur at $2 \mathrm{~F} 1 \pm \mathrm{F} 2$ and $2 \mathrm{~F} 2 \pm \mathrm{F} 1$. Out of these 2 pairs, the ones occurring at $2 \mathrm{~F} 1-\mathrm{F} 2$ and 2F2-F1 can possibly occur within the desired bandwidth range and thus affect the linearity and output of the device. These signals per theory have a slope in ration of 3:1 as compared to the fundamental tones, which would have a slope of 1:1 within the linear range. The IIP3 point or the third order input intercept point is the theoretical point at which the inter-modulation products and the fundamental tones meet and are at the same input power level. Thus the IIP3 is a good measure of the linearity of the USRP. The higher the IIP3, more liner is the device. Or in other words the device is linear over a longer range of amplitude of the input signal if it has a higher IIP3 value [22] [31] [32].

If the intercept from the point of convergence, is taken on the input power scale ( $\mathrm{X}$ axis) we would get the value of the Input Intercept point. If taken on the output power (Y axis) we get the Output Intercept point or OIP3. Both OIP3 and IIP3 are key to getting information about the spurious free dynamic linear range of the device and higher the values of IIP3 and OIP3, then it 
implies that the inter-modulation products are weaker and would not interfere with the main operating signal [22] [31] [33]

Analytically the IIP3 point can be calculated using the formula [32]:

$I I P 3=P_{\text {in }}+\frac{P_{F 1}-P I M D}{2}$

As $P_{i n}$ and $P_{\text {in }}$ and $P_{F 1} / P_{F 2}$ are 1:1 proportional, it could also be written as:

$I I P 3=P_{F 2}+\frac{P F 1-P I M D}{2}$

$P_{F 1}$ and $P_{F 2}$ are interchangeable provided they show equal amplitudes. It applies for IMD products 2 F2-F1 and 2 F1-F2 frequencies as well.

The OIP3 is simply given as:

$$
O I P 3=I I P 3+\text { Gain }
$$

Another important thing to note is that point of convergence of fundamental and intermodulation products' power level is purely theoretical, as the device is never pushed to that point. It would get severely damaged if done so, and moreover there would be internal system breaks placed to avoid this i.e. the device would saturate [32].

One more important relation is that of $1-d B C P$ and IIP3. It is as given below [32]:

$$
I P 3=9.6 d B+1-d B C P
$$


where IP3 is the Third Order Intercept Point [31]. So generally the $3^{\text {rd }}$ order intercept point should be $\sim 10 \mathrm{~dB}$ higher than the $1 \mathrm{~dB}$ compression point of the device. The complete test procedure is described in Chapter 4 along with the test results. The basic block diagram of the hardware setup is as shown in the Figure 3.5.

As shown two signal generators were used and to produce the two fundamental tones and their output connected was combined using a power combiner (having a loss of $\sim 3 \mathrm{~dB}$ ). The output of this combiner was given to the RX (RF2) port of the USRP device. The USRP device was connected to the host laptop wherein the UHD_FFT.grc flow graph was executed to get the real time spectrum. The output was measured at the two fundamental tones F1 and F2 and the inter-mod frequencies of 2F1-F2 and 2F2-F1. The output from the power combiner was also fed to the spectrum analyzer for initial loss measurements i.e. to check the actual input power to the USRP RX port. The readings were tabulated and a graph was plotted using readings at F1 and 2F2-F1 (can be any combination of the two pairs of frequencies as long as they are equal). As mentioned before, the $3^{\text {rd }}$ intercept point is theoretical and hence the point was obtained through the intersection point of the trend lines of the measurements at the frequencies. 


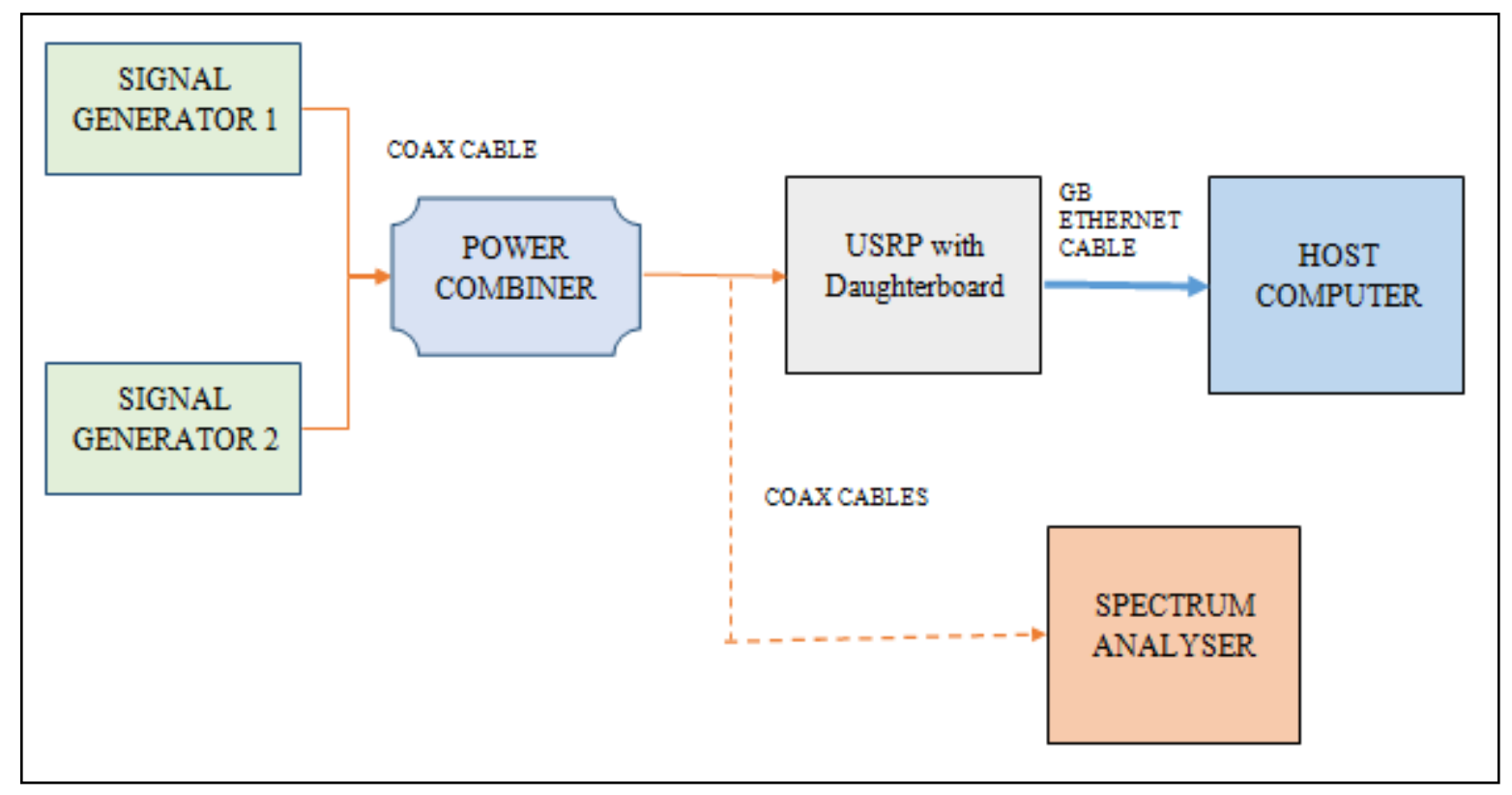

Figure 3.5 Block Diagram for IIP3 Test

The OIP3 point of the device (USRPN210+WBX) was found to be around $1.96 \mathrm{dBm}$ while the IIP3 was found to be $\sim 2.3 \mathrm{dBm}$ graphically at $400 \mathrm{MHz}$. This was using the calibration value found using the $1 \mathrm{dBC}$ point test of 35.5 as the difference between the UHD_FFT.grc plot reading and the actual spectrum analyzer reading for a given input power from the Agilent Signal source at $400 \mathrm{MHz}$. However in this test the power combiner apparatus leads to a loss of $\sim 4 \mathrm{dBm}$ and hence the effective gain or calibration factor used is $=35.5+4=31.5 \mathrm{dBm}$. This was for test conducted at $400 \mathrm{MHz}$. The readings of IMD @ $400.7 \mathrm{MHz}$ were calibrated with just the loss of $4 \mathrm{dBm}$ as other correction factor of 35.5 is valid only in linear range.

Using the relation that IP3 needs to be around $10 \mathrm{dBm}$ higher than $1 \mathrm{dBCP}$, we can now estimate the I-dBC point at $400 \mathrm{MHz}$. At $400 \mathrm{MHz}$, IIP3 is $2.8 \mathrm{dBm}$, this implies $1 \mathrm{dBCP}$ has to be approx. $2.3-10=-7.7 \mathrm{dBm}$, which seems a likely value as the USRPN210 displays linear 
characteristics until $-10 \mathrm{dBm}$. Using the OIP3 value at $400 \mathrm{MHz}$ of $1.96 \mathrm{dBm}$ we get the $1 \mathrm{dBC}$ point as $1.96-10=-8.04 . \mathrm{dBm}$ which again close to the estimate and predicted value. Similarly at $900 \mathrm{MHz}$ the IIP3 was found to be $5.6 \mathrm{dBm}$. Hence the $1 \mathrm{dBC}$ point can be estimated to be around $5.6-10=-4.4 \mathrm{dBm}$.

Analytically also, the average IIP3 found for 8 devices is found to be approximately 0.25 @ $400 \mathrm{MHz}$ and $3.95 \mathrm{dBm}$ at $900 \mathrm{MHz}$ (see Chapter 4). There were large deviations (ranging between -2.51 to 2.58 ) in readings of certain USRPN210 devices, which may be attributed to the individualistic conditions of the device and to the fact that readings were prone to human error. At $1800 \mathrm{MHz}$ for USRPN210 device with WBX board, the IIP3 point was found to be approximately $-2.46 \mathrm{dBm}$ analytically, and hence $1 \mathrm{dBCP}$ can be estimated to be approximately $2.46-10=-12.46 \mathrm{dBm}$. This is summarized in table 3.2 below.

Table 3.2 Analytical IIP3 Calculation at different Frequencies

\begin{tabular}{|c|c|c|c|c|c|c|c|}
\hline \multirow[t]{2}{*}{ USRP Type } & \multirow[t]{2}{*}{$\begin{array}{l}\text { RX Gain } \\
\text { Setting on } \\
\text { UHD_FFT } \\
\text { GUI (dB) }\end{array}$} & \multirow[t]{2}{*}{$\begin{array}{l}\text { Center } \\
\text { Frequency }\end{array}$} & \multirow{2}{*}{$\begin{array}{c}\text { Signal } \\
\text { generator } \\
\text { reading } \\
\text { when the } \\
\text { IMD } \\
\text { reading is } \\
\text { taken }\end{array}$} & \multicolumn{3}{|c|}{$\begin{array}{l}\text { O/p Reading on UHD_FFT } \\
\text { GUI at the fundamental and } \\
\text { IMD frequencies (dB) }\end{array}$} & \multirow[t]{2}{*}{$\begin{array}{c}\text { IIP3 = (F2-IM1)/2+ } \\
(\text { F1- Correction } \\
\text { Factor+loss) } \\
(\mathrm{dBm})\end{array}$} \\
\hline & & & & F1 & F2 & 2F2-F1 & \\
\hline $\begin{array}{l}\text { USRPN210 + WBX } \\
\text { board (VT } \\
361926)\end{array}$ & 0 & $400 \mathrm{MHz}$ & -33 & -1.984 & -2.054 & -68.81 & -0.141 \\
\hline $\begin{array}{l}\text { USRPN210 + WBX } \\
\text { board (VT 361926) }\end{array}$ & 0 & $900 \mathrm{MHz}$ & -23.5 & -8.736 & -8.639 & -84.934 & 2.29 \\
\hline $\begin{array}{l}\text { USRPN210 + WBX } \\
\text { board (VT 361926) }\end{array}$ & 0 & $1800 \mathrm{MHz}$ & -10 & 2.689 & 3.013 & -26.01 & -2.46 \\
\hline
\end{tabular}




\subsubsection{The Received Power Stability Test}

Though power transmitted by USRP is an important metric to be measured, the stability of the power received by the USRP in the allowed range of frequencies is also of much importance to judge the performance of the USRP. The received power seen on UHD_FFT.grc for a set power taken as input at RF2 Port (RX) should be the same at different frequencies. If there is a variation in the power as seen on UHD_FFT.grc for same input power on RX port across frequencies then it might imply that the internal processing done within the USRP is based on some frequency range, which is not expected and as per the normal understanding of a radio device. This test was done with the similar setup same as shown in figure 3.6. The USRP was connected to host PC via gigabit Ethernet cable while the input from Signal generator is fed to the RF2 (RX) port via a coaxial cable. The UHD_FFT.py program is executed to get the received signal spectrum. The power at the RF2 port maintained constant while the frequency is varied from $50 \mathrm{MHz}$ to $2.2 \mathrm{GHz}$ (the lowest to highest frequency the WBX daughterboard). For first few USRPs it is varied in steps of $50 \mathrm{MHz}$ and then later in steps of $100 \mathrm{MHz}$ (after not much variations was seen). The output power throughout the operational frequency range of the USRP daughter board is noted. The output power shows variation (a dip of about $1 \mathrm{~dB}$ ) with change in frequency $(100 \mathrm{MHz})$. However this characteristic is common across the different input power of $-10,-30$ and $-60 \mathrm{dBm}$. This shows that internal processing of USRP is not frequency specific, but common for the entire operational range. 


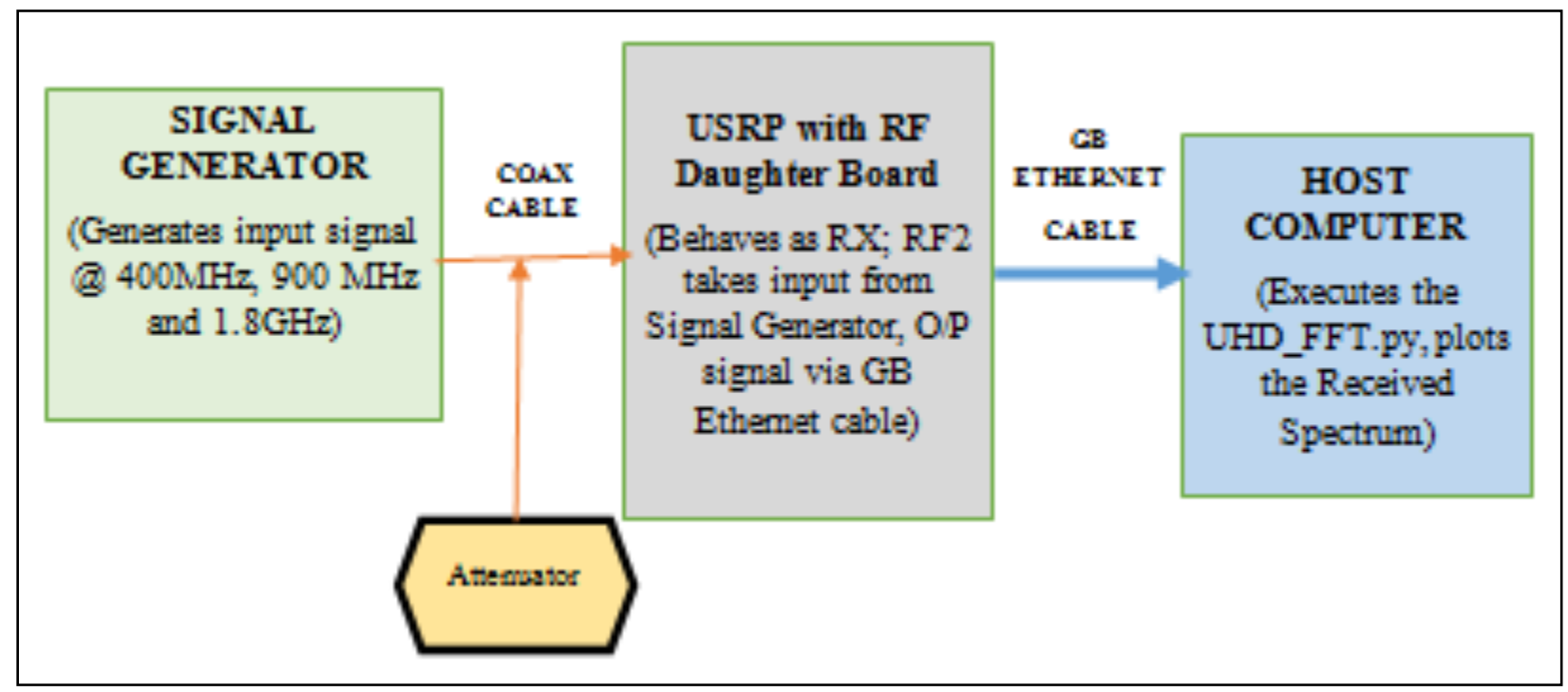

Figure 3.6 Block Diagram of Received Power Stability Test

This test is done for an input power of $-10 \mathrm{dBm},-30 \mathrm{dBm}$ and $-60 \mathrm{dBm}$ at the RF2 port. Variations seen with change in input power at RF2 port can be termed as an almost linear decrease in output power with increase in frequency of operation. The readings are tabulated and the entire procedure of the test with the results is described in Chapter 4. The basic block diagram of the hardware setup is as shown Figure 3.6.

It is seen that (refer table 3.3) in change in frequency in steps of $500 \mathrm{MHz}$ almost produced a 3-4 $\mathrm{dB}$ dip in output power noted on UHD_FFT.grc plot tuned to the center frequency. This seems consistent with the readings obtained during the $1 \mathrm{dBC}$ point test where the mean correction factors at $400 \mathrm{MHz}, 900 \mathrm{MHz}$ and $1800 \mathrm{MHz}$ are 35.5,31.2 and 23.5 showing a difference of $\sim 4 \mathrm{~dB}$ (between 400 and $900 \mathrm{MHz}$ ) and $\sim 8 \mathrm{~dB}$ (between 900 and 1800 $\mathrm{MHz}$ ). This information would be useful to predict the correctness of any value measured on UHD_FFT.grc with the frequency range of WBX board at/near these input power levels. For e.g. 
in experiments that require test to be carried out over the entire frequency range of the board over long periods in terms of number of days.

Table 3.3 Amplitude Change across different Devices and Frequencies steps

\begin{tabular}{|c|c|c|c|c|c|c|}
\hline \multirow{2}{*}{ USRP Type } & \multirow{2}{*}{$\begin{array}{c}\text { Input } \\
\text { Power set } \\
\text { (dBm) }\end{array}$} & \multicolumn{5}{|c|}{ Change in Pout on UHD_FFT across frequency in steps of $500 \mathrm{MHz}(\mathrm{dB})$} \\
\hline & & $\begin{array}{c}100 \mathrm{MHz} \text { to } \\
500 \mathrm{MHz}\end{array}$ & $\begin{array}{l}500 \mathrm{MHz} \text { to } \\
1000 \mathrm{MHz}\end{array}$ & $\begin{array}{c}1000 \mathrm{MHz} \text { to } \\
1500 \mathrm{MHz}\end{array}$ & $\begin{array}{l}1500 \mathrm{MHz} \text { to } \\
2000 \mathrm{MHz}\end{array}$ & $\begin{array}{c}2000 \mathrm{MHz} \text { to } \\
2500 \mathrm{MHz}\end{array}$ \\
\hline \multirow{3}{*}{$\begin{array}{l}\text { USRPN210 } \\
+ \text { WBX }\end{array}$} & -60 & 2.81 & 4.78 & 3.65 & 2.42 & $\mathrm{~N} / \mathrm{A}$ \\
\hline & -30 & 2.91 & 4.64 & 3.79 & 2.29 & $\mathrm{~N} / \mathrm{A}$ \\
\hline & -10 & 3.02 & 5.01 & 3.43 & 2.49 & N/A \\
\hline \multirow{3}{*}{$\begin{array}{l}\text { USRPN210 } \\
+ \text { SBX }\end{array}$} & -60 & $\mathrm{~N} / \mathrm{A}$ & 1.85 & 3.49 & 2.00 & 1.40 \\
\hline & -30 & N/A & 1.79 & 3.15 & 2.90 & 4.58 \\
\hline & -10 & $\mathrm{~N} / \mathrm{A}$ & 1.69 & 3.23 & 2.07 & 5.83 \\
\hline
\end{tabular}

\subsubsection{The Transmitted Signal RF Carrier Frequency Stability Test}

The operating frequency of a device is generally the RF carrier frequency on to which the baseband signal is modulated upon. In any radio device, the operating on RF spectrum would encompass a series of components like mixer, PLL, VCO which are bound to produce oscillations from the central operating frequency value due to various possible noises like thermal noise, jitter noise, mechanical shocks. These in turn tend to make the carrier frequency deviate from its central value. In a wideband operation, the deviations of up to 1-3 KHz may not make much of a difference in the output produced in the frequency dependent operations (like WCDMA etc.). However, in a narrow band operation, even a deviation of 1-3 KHz would create 
a large difference in the output produced. Hence measure of operating frequency stability is a very important metric [11] [24].

The complete test procedure is described in chapter 4 along with the results. The experiment setup is as shown in Figure 3.7. The USRP is connected to the host computer via the gigabit Ethernet cable while the RF1 (TX/RX) port of USRP is connected to the spectrum Analyzer. The UHD_SIGGEN.grc is the pre-defined signal generator application for GNU Radio. It is used to generate a digital signal samples at desired frequency with the desired amplitude. The spectrum Analyzer allows to measure the RF carrier frequency up to $10 \mathrm{e}-7 \mathrm{~Hz}$ in value.

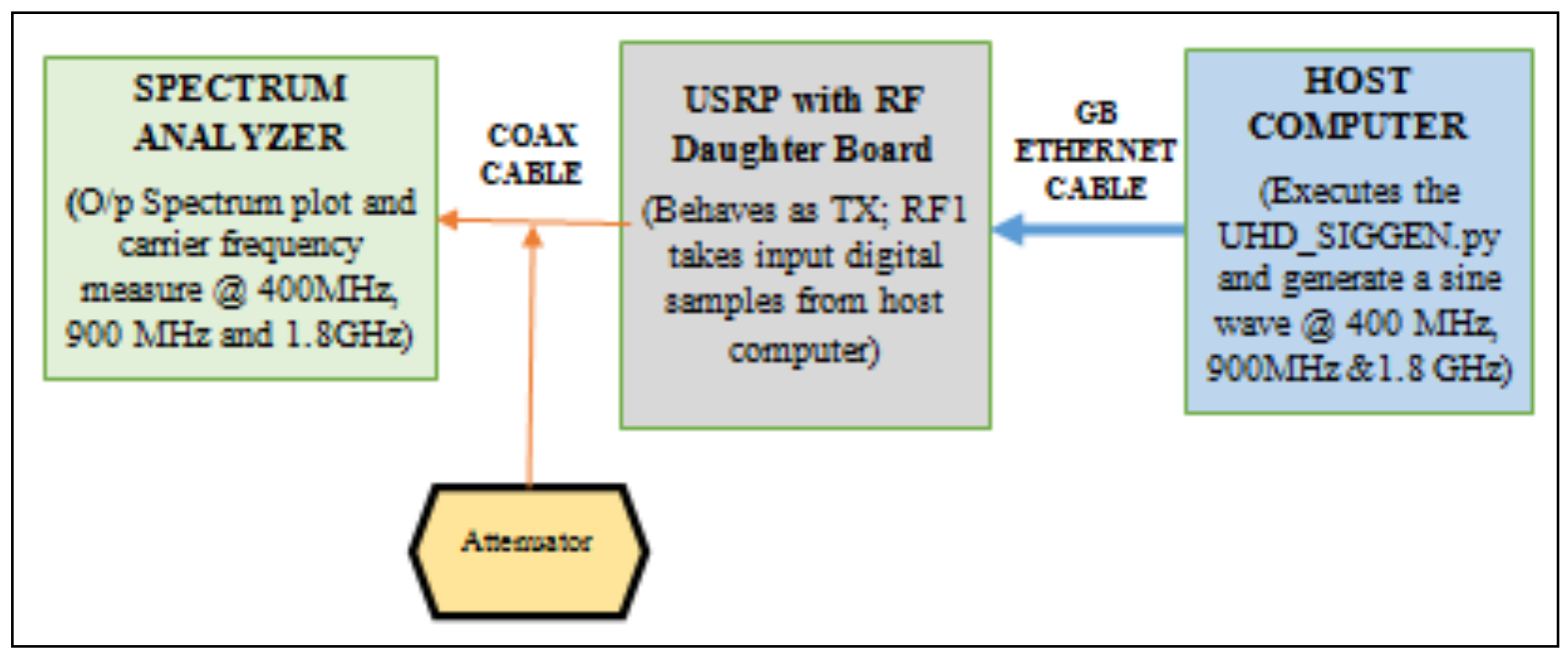

Figure 3.7 Block Diagram of TX Frequency Stability Test

The measure of carrier frequency as per the spectrum analyzer is noted for period of 1 hour at every 2 minutes. The experiment is done for $400 \mathrm{MHz}, 900 \mathrm{MHz}$ and $1.8 \mathrm{GHz}$. As shown 
in the table 3.4 below, the USRPN210(s) with WBX boards displayed very small deviations from its expected and set frequency of operation.

For $400 \mathrm{MHz}$, the UHD_SIGGEN.py was generated a sine wave signal at $400.1 \mathrm{MHz}$ by modulating a $100 \mathrm{KHz}$ baseband signal at a RF frequency of $400 \mathrm{MHz}$. The TX gain in UHD_SIGGEN.py was set to $0 \mathrm{~dB}$. The output from the host computer in form of digital samples was fed to the USRP via gigabit Ethernet cable, which was then transmitted by the USRP from the RF1 port. The output of the RF1 port was fed to the spectrum analyzer via a coaxial cable.

For $900 \mathrm{MHz}$ and $1.8 \mathrm{GHz}$ operations the same procedure was repeated except that the RF frequency was set to $900 \mathrm{MHz}$ and $1.8 \mathrm{GHz}$ respectively. As seen in table 3.4 , the average frequency deviation at $400 \mathrm{MHz}, 900 \mathrm{MHz}$ and $1.8 \mathrm{GHz}$ is negligible and negative. For most part the USRPN210 devices are seem to be pretty stable devices in terms of transmitted signal frequency.

Table 3.4 Transmitted Signal Frequency Stability Test Measurements

\begin{tabular}{|c|c|c|c|c|c|c|}
\hline \multirow[t]{2}{*}{ USRP Type } & \multicolumn{3}{|c|}{$\begin{array}{c}\text { Average Frequency Deviation from the } \\
\text { Expected Frequency i.e. Actual measured } \\
\text { TX frequency - Expected TX frequency on } \\
\text { spectrum analyzer (over a period of } 1 \\
\text { hour) ( } \mathrm{MHz} \text { ) }\end{array}$} & \multicolumn{3}{|c|}{ 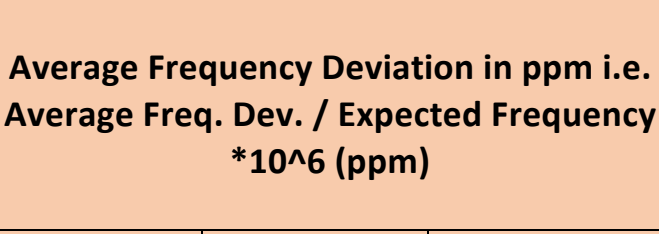 } \\
\hline & $400 \mathrm{MHz}$ & $900 \mathrm{MHz}$ & $1800 \mathrm{MHz}$ & $400 \mathrm{MHz}$ & $900 \mathrm{MHz}$ & $1800 \mathrm{MHz}$ \\
\hline $\begin{array}{l}\text { USRPN210 + WBX } \\
\text { board }\end{array}$ & $-1.27 E-04$ & $1.81 \mathrm{E}-03$ & $-4.83 E-04$ & -0.32 & -0.09 & -0.27 \\
\hline $\begin{array}{l}\text { USRPN210 + SBX } \\
\text { board }\end{array}$ & $2.12 \mathrm{E}-03$ & $6.91 \mathrm{E}-04$ & $-1.36 \mathrm{E}-03$ & 5.31 & 0.77 & -0.76 \\
\hline USRP2 + WBX Board & $3.22 \mathrm{E}-03$ & 8.53E-03 & 1.70E-02 & 8.05 & 9.47 & 0.01 \\
\hline
\end{tabular}




\subsubsection{The Transmitted Signal Output Amplitude Stability Test}

The amplitude of transmitted signal and its stability is also a metric that was tested in lines with the frequency stability. This was to check two things: (1) If the frequency variations produced any variations in the transmitted signal power like any spurious peaks; and (2) If the signal power dropped after a certain time when it was generated by a software program as digital samples.

The experimental setup for this test was similar to the one for frequency stability test whereby the RF1 (TX) port was connected to the spectrum analyzer while the input signal generated by the UHD_SIGGEN.py program on the host computer was sent to the USRP via the gigabit Ethernet cable. The output signal amplitude was motored over 1 hour and measurements done every 2 minutes. The test was conducted at $400 \mathrm{MHz}, 900 \mathrm{MHz}$ and $1.8 \mathrm{GHz}$ as RF carrier frequencies $(1 \mathrm{KHz}$ baseband signal modulated at RF carrier frequency to get a modulated sinusoidal wave as output at $400.1 \mathrm{MHz}, 900.1 \mathrm{MHz}$ and $1.8001 \mathrm{MHz}$ ) The test was conducted for a sample of 10 USRPN210(s) with WBX board. For the purpose of comparison, the test was also conducted for a USRPN210 with SBX board and a USRP2 with WBX board to check if there are any major variations created due to the motherboard or SBX board configuration.

The experimental procedure is fully detailed in Chapter 4 and the experimental setup is as shown in Figure 3.8 below. The TX gain is set as $0 \mathrm{~dB}$ for the experiment. An external attenuation of $30 \mathrm{~dB}$ was applied to avoid any damage to the spectrum analyzer. 


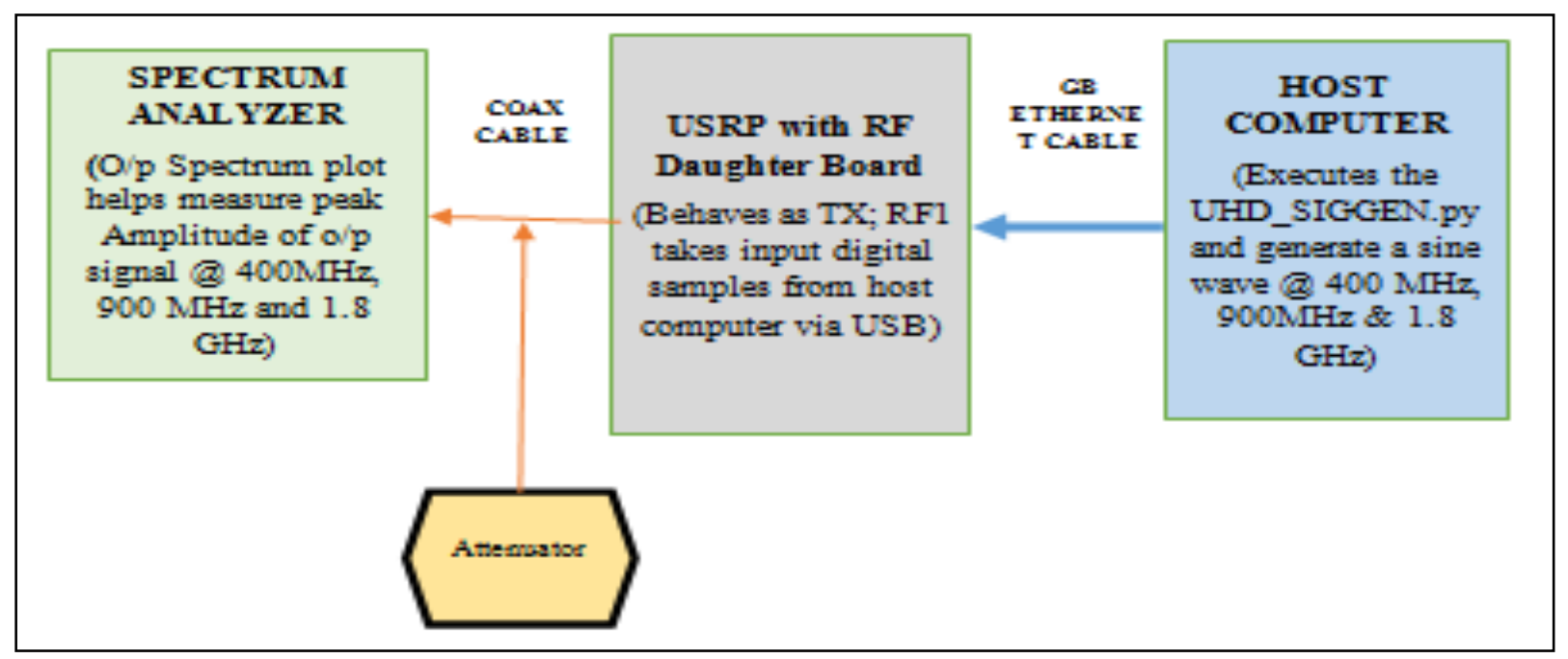

Figure 3.8 Block Diagram of TX Amplitude Stability Test

The amplitude was found to be considerably stable for the USRPN210(s) over the period of 1 hour of observation. The UHD_SIGGEN was run with parameters of Amplitude $=1$, TX gain $=0$ to produce sinusoidal wave samples at 1 MSamples per sec rate (external attenuation of $30 \mathrm{~dB}$ applied). The mean amplitude observed from the reading and the standard deviations from the mean have been tabulated for $400 \mathrm{MHZ}, 900 \mathrm{MHZ}, 1.8 \mathrm{GHz}$ in table 3.5. This was repeated for USRPN210 +SBX board and USRP2 +WBX board. The SBX and board seem to produce markedly different output power levels than WBX board.

If only the TX gain setting was changed to $30 \mathrm{~dB}$, the output was as observed to be 9.33dBm at $400 \mathrm{MHz},-11.75 \mathrm{dBm}$ at $900 \mathrm{MHz}$, and $-15.91 . \mathrm{dBm}$ at $1800 \mathrm{MHz}$. When the experiment was conducted with an SBX board, the output signal amplitude change is not consistent with the one seen with WBX board, though the amplitudes are stable for the time period of 1 hour. Same is the case with USRP2 with WBX board. 
Table 3.5 Transmitted Signal Amplitude Stability Test Measurements

\begin{tabular}{|c|c|c|c|c|c|c|}
\hline \multirow[t]{2}{*}{ USRP Type } & \multicolumn{3}{|c|}{$\begin{array}{l}\text { Average Output Power observed for the } \\
\text { transmitted signal }(A m p l .=1, \text { TX Gain = } \\
0 \text {, external attn. }=30)(\mathrm{dBm})\end{array}$} & \multicolumn{3}{|c|}{$\begin{array}{l}\text { Amplitude Deviation from the mean and } \\
\text { the confidence interval limit of the } \\
\text { transmitted signal }(\mathrm{Ampl}=1, \mathrm{TX} \text { Gain }=0) \\
(\mathrm{dBm})\end{array}$} \\
\hline & $400 \mathrm{MHz}$ & $900 \mathrm{MHz}$ & $1800 \mathrm{MHz}$ & $400 \mathrm{MHz}$ & $900 \mathrm{MHz}$ & $1800 \mathrm{MHz}$ \\
\hline $\begin{array}{l}\text { USRPN210 + WBX } \\
\text { board }\end{array}$ & -35.74 & -39.21 & -47.39 & $0.14,0.087$ & $0.109,0.333$ & $0.534,0.4$ \\
\hline $\begin{array}{l}\text { USRPN210 + SBX } \\
\text { board }\end{array}$ & -56.29 & -57.18 & -62.79 & $0.26,0.504$ & $0.261,0.513$ & $0.547,1.072$ \\
\hline USRP2 + WBX Board & -41.45 & -37.92 & -38.41 & $0.89,1.74$ & $0.16,0.311$ & $0.233,0.456$ \\
\hline
\end{tabular}

When a USRPN210 with WBX board is given input from a GNU Radio flow graph instead of the UHD_SIggen.py, the output amplitudes do not follow the trend seen previously and with external attenuation $=60 \mathrm{~dB}$, gives the output power as $-53.574 \mathrm{dBm} @ 400 \mathrm{MHz}$, -48.801 dBm@900 MHz and -51.3880 dBm@1800 MHz.

\subsubsection{Transmitted Signal Variation with TX Gain Test}

The gain range of a daughter board is the max allowable gain for the transmitted signal. This in turns affects the maximum power that can be transmitted by the device under the given set of conditions. Observing the variation of the output power with respect to the transmitter gain would help understand the transmitted power from these USRP devices, which by fact are said to be un-calibrated devices undergoing various stages of amplification before being fed to the DAC [1] [15]. This would also enable learning of the linear range of the device when behaving as a transmitter. 


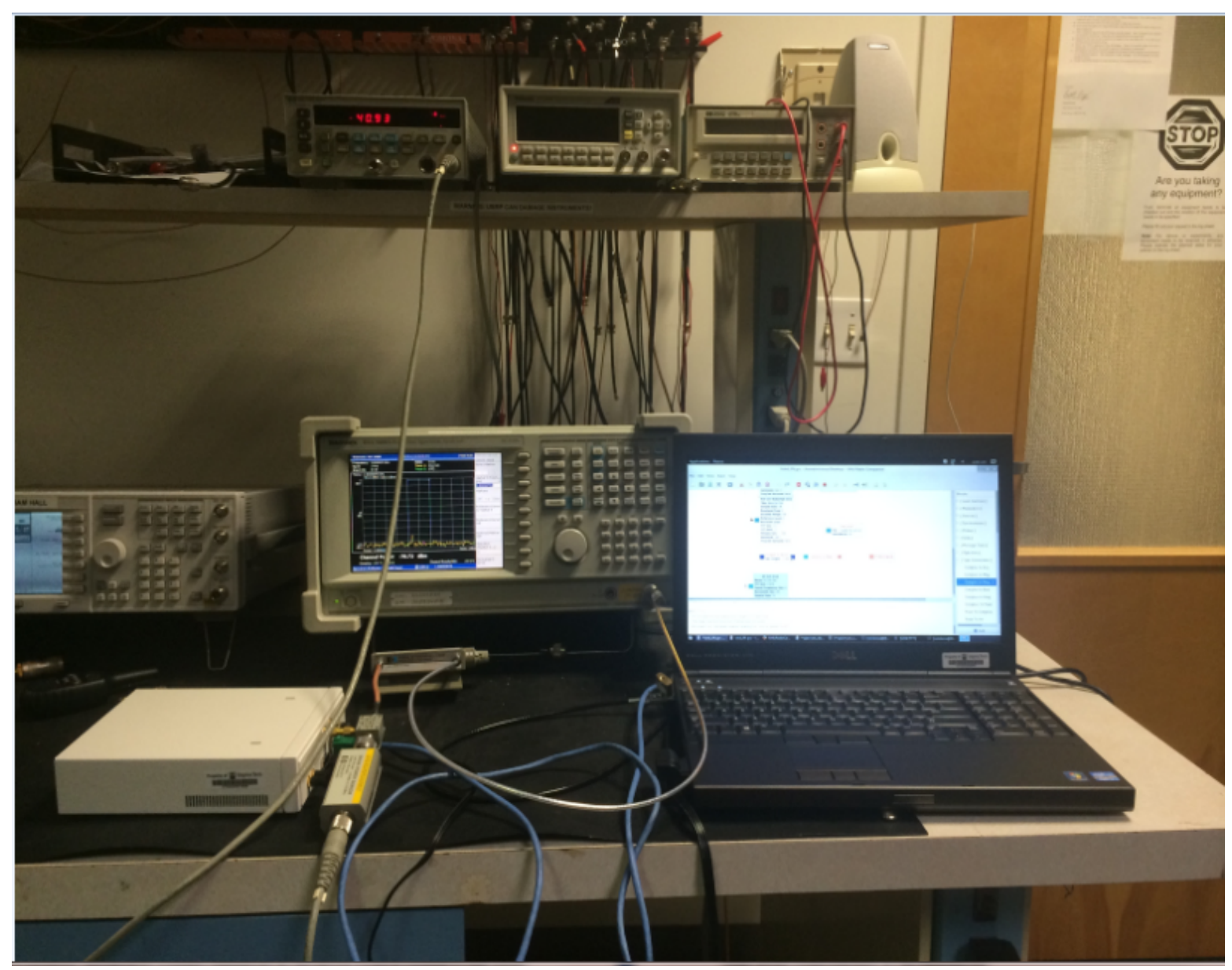

Figure 3.9 Laboratory Setup for TX Characterization

The setup for this test is similar to most of the Transmitter characterizations test. The device is connected to the host laptop using a gigabit Ethernet connection. The TX (RF1) port's output is fed to a power splitter. One output port of the power splitter fed the spectrum analyzer while the second output port was connected to a power meter. A power meter enables more accurate reading of the power as it measures the power over the entire signal bandwidth. The measured reading were tabulated and studied to understand the effect of gain on the output power and linearity of the device. Single tone and two-tone tests were conducted for this purpose 
on the USRPN210 device [33]with WBX board and USRPN210 device with SBX board, as this is mainly dependent on the gain of the daughter board.

Table 3.6 Transmitter Signal Variation with TX Gain Test Measurements

\begin{tabular}{|c|c|c|c|c|c|c|}
\hline \multirow[t]{2}{*}{ USRP \# } & \multicolumn{3}{|c|}{$\begin{array}{l}\text { Gain Value denoting the start of } \\
\text { Compression Region for the Single } \\
\text { Tone transmitted signal (Ampl } \\
=0.707)(\mathrm{dBm})\end{array}$} & \multicolumn{3}{|c|}{$\begin{array}{l}\text { Gain Value denoting the start of } \\
\text { IMD products near }-30 \mathrm{dBc} \text { for the } \\
\text { Two Tone transmitted signal (Ampl } \\
=0.354)(\mathrm{dBm})\end{array}$} \\
\hline & $400 \mathrm{MHz}$ & $900 \mathrm{MHz}$ & $1800 \mathrm{MHz}$ & $400 \mathrm{MHz}$ & $900 \mathrm{MHz}$ & $1800 \mathrm{MHz}$ \\
\hline USRPN210 + WBX board & 30 & 30 & 31 & 31 & 31 & 31 \\
\hline USRPN210 + SBX board & 25 & 25 & 25 & 25 & 28 & 31 \\
\hline USRP2 + WBX board & $4-25$ & $5-25$ & $5-25$ & $2-10$ & $8-10$ & $5-10$ \\
\hline
\end{tabular}

With the single tone test, gain compressions were visible at around $30 \mathrm{~dB}$ TX gain at all 3 frequencies for USRPN210+WBX device, while for SBX board output gain compression was visible at $25 \mathrm{~dB}$ of gain itself at 400, $900 \mathrm{MHz}$ and $1800 \mathrm{MHz}$. Similarly inter-modulation products power with respect to the main carrier did not seem to go beyond $-30 \mathrm{~dB}$ for USRPN210+WBX devices. Hence the maximum gain $(31 \mathrm{~dB})$ is set as end-point for linear characteristics of this device (USRPN210+WBX). But for the SBX board IMD of about -30 dBc could be seen by $25 \mathrm{~dB}$ and $28 \mathrm{~dB}$ at 400 and $900 \mathrm{MHz}$ respectively. For USRP2 with WBX board, it was seen that until $5 \mathrm{~dB}$ value gain did not produce any effect on the output power level. Output power started increasing only from $5 \mathrm{~dB}$ onward at all 3 frequencies. Hence for 
USRP2 alone the linear range varies from $5 \mathrm{~dB}$ to $25 \mathrm{~dB}$ gain, where the output power level starts saturating. Similar conclusions (refer table 3.6) are drawn for the USRPN210+SBX wherein linear range varies from $0 \mathrm{~dB}$ gain to $25 \mathrm{~dB}$ at all 3 frequenices. 


\section{TESTING AND RESULTS}

This chapter details the procedure used for performing the various experiments for this research. Since most of the experiments were conducted manually, considerations for human error and laboratory/environmental conditions need to be made for the results obtained. As mentioned before in the earlier chapters, for most of the experiments a sample of 10 USRPN210s were used to observe the behavior and then averaged to get the sample mean as the representative measurement.

\subsection{RX Characterization}

The below picture depicts the setup for receiver side tests conducted. The blue cable being the gigabit Ethernet connects the host laptop and DUT. The RX port is connected to the signal generator using coaxial cable of around $500 \mathrm{~cm}$. The black cable is the power cable for the USRPN210 providing a DC voltage of $6 \mathrm{~V}$. The spectrum analyzer shown is the Tektronix Spectrum Analyzer (RSA 3408, operating frequency range: DC - $6 \mathrm{GHz}$ ) while the Agilent Signal generator (E4438C, operating frequency range: $250 \mathrm{kHz}$ to $6 \mathrm{GHz}$ ) was used for most of the $\mathrm{RX}$ side experiments operating at a frequency range of and providing power from $-136 \mathrm{dBm}$ to $+20 \mathrm{dBm}$. For IIP3 Test, the HP signal generator is also used as the second input signal source. 


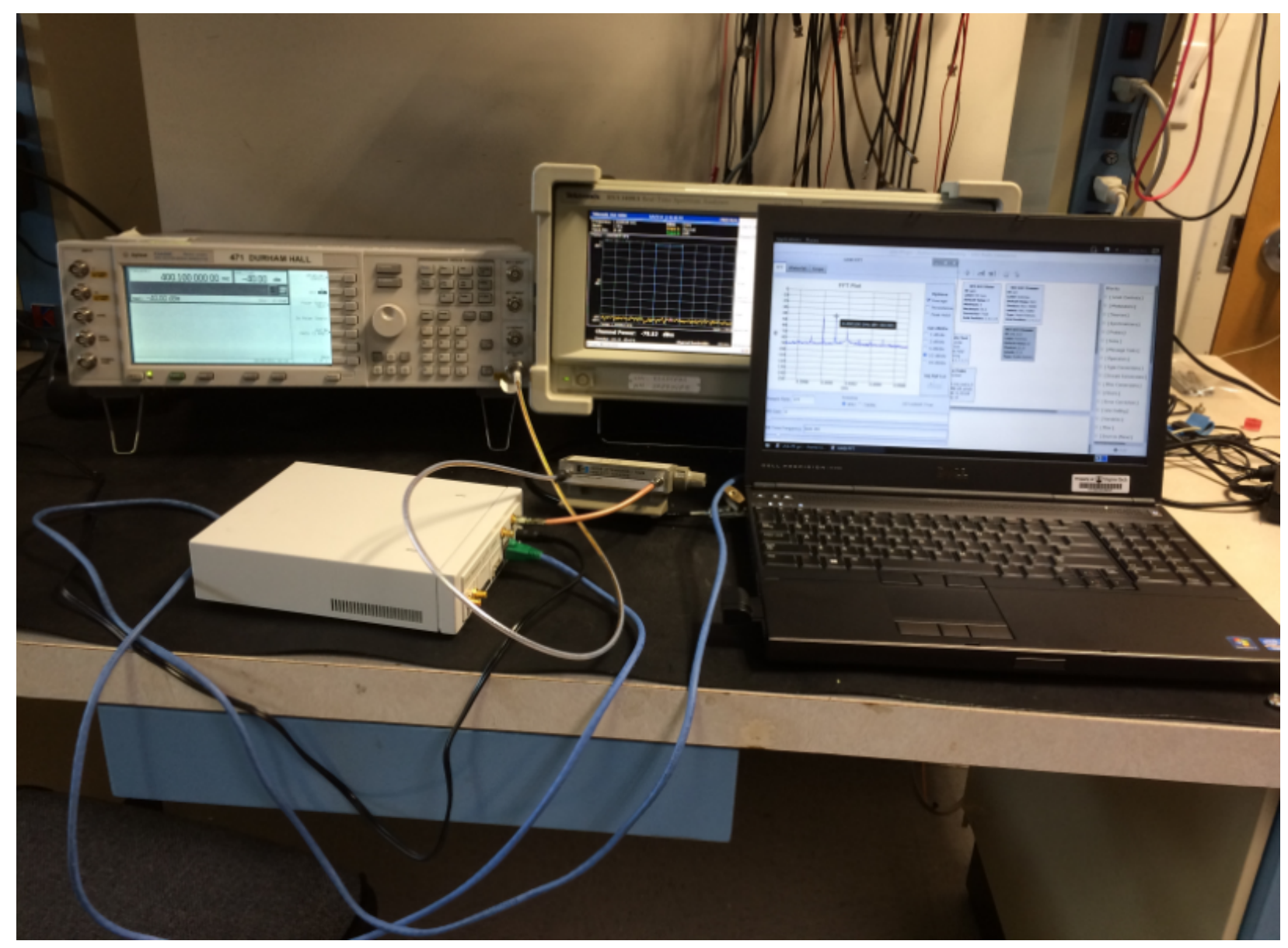

Figure 4.1 Laboratory Setup for 1-dB Compression Point and RX Power Stability Test

\subsection{1 $1 \mathrm{~dB}$ Compression Point Test Procedure}

1. Connect USRPN210 device to HOST PC using the gigabit Ethernet cable (blue in the picture)

2. Connect the AGILENT SIGNAL GENERATOR Power Outlet to USRP N210 Rx port (RF2) as input via coax cable. 
3. Executing the UHD_FFT.grc flow graph set at the desired frequency on the host PC generates the received spectrum plot.

4. Here the measurements are done @ $400.7 \mathrm{MHz}$ as this is one of the frequencies where the CORNET USRP operations are valid and licensed, and also this is the frequency where harmonics are generated for fundamental frequencies [34] of 400.1MHz and 400.4MHz.

5. For the first USRP device under test the Power in signal generator is set right from $-115 \mathrm{dBm}$ -where we get the Minimum detectable signal (Noise floor is found to be $\sim 120 \mathrm{dBm}$ ) and increased thereafter by $10 \mathrm{dBm}$ until $-10 \mathrm{dBm}$ is the signal power displayed on the signal generator.

6. The output power level seen on the UHD_FFT plot is noted per increase in input signal power.

7. From $-20 \mathrm{dBm}$ to $-10 \mathrm{dBm}$ region, watch for any gain compressions.

8. The Gain factor for each data set of USRP is calculated as:

Gain $(\mathrm{dB})=$ Pout $($ as seen on UHD_FFT.py plot $)-$ Pin (Power value seen on signal generator when the reading is taken)

9. For each USRP, the Gain Vs Pin is plotted, which graphically/visually enables to see any dip in gain for increase in Pin.

10. However until $-10 \mathrm{dBm}$ of input power, no dips were seen. The gain factor was seen to trend around a constant value of $\sim 35 \mathrm{~dB}$.

11. The same experiment is repeated for $900 . \mathrm{Mhz}$ and $1.800 \mathrm{MHz}$ frequencies for the set of 10 USRPs. 
12. This experiment was also repeated for a USRP2 with WBX board and USRPN210 with SBX board.

13. The readings were tabulated (table 4.1), and the mean gain for each frequency was noted as the correction factor. This was taken as the correction factor as the trend was similar across 10 devices of same type.

Table 4.1 Readings for 1-dBCP Test (Correction Factors derived)

\begin{tabular}{|c|c|c|c|c|c|c|}
\hline \multirow[t]{2}{*}{ USRP Type } & \multirow[t]{2}{*}{$\begin{array}{c}\text { Type of } \\
\text { DB }\end{array}$} & \multirow[t]{2}{*}{ Mboard } & \multirow[t]{2}{*}{ Dboard } & \multicolumn{3}{|c|}{$\begin{array}{l}\text { Average Received Power offset b/w } \\
\text { UHD_FFT and Spectrum Analyzer } \\
\text { (dB) }\end{array}$} \\
\hline & & & & $400 \mathrm{MHz}$ & $900 \mathrm{MHz}$ & $1800 \mathrm{MHz}$ \\
\hline \multicolumn{7}{|l|}{$\begin{array}{l}\text { USRPN210(s) with } \\
\text { WBX daughter } \\
\text { boards }\end{array}$} \\
\hline VT361926 & WBX & F3EDFC & F3C95B & 34.77 & 29.73 & 20.51 \\
\hline VT361927 & WBX & F3ECE3 & F3C97F & 35.17 & 30.70 & 22.67 \\
\hline VT361928 & WBX & F3ECE1 & F3E5DC & 34.91 & 30.81 & 23.22 \\
\hline VT361929 & WBX & F3EE3D & F3E5E6 & 35.65 & 31.87 & 24.86 \\
\hline VT361930 & WBX & F3EE51 & F35536 & 35.32 & 31.05 & 24.54 \\
\hline VT361931 & WBX & F3EE2C & F3E5E4 & 35.64 & 31.70 & 23.79 \\
\hline VT361932 & WBX & F38078 & F3C1F7 & 36.22 & 31.65 & 22.13 \\
\hline VT361933 & WBX & F3C271 & F37488 & 35.55 & 31.40 & 24.65 \\
\hline VT361936 & WBX & F3EE61 & F3E5CE & 35.76 & 31.58 & 24.51 \\
\hline VT361937 & WBX & F3EE4B & F35541 & 36.40 & 31.92 & 24.79 \\
\hline Average & & & & 35.54 & 31.24 & 23.57 \\
\hline Standard Deviation & & & & 0.52 & 0.68 & 1.44 \\
\hline $\begin{array}{c}\text { Confidence Interval } \\
95 \% \text { (Margin of Error) }\end{array}$ & & & & 0.32 & 0.42 & 0.89 \\
\hline RMS off-set & & & & 35.54 & 31.25 & 23.61 \\
\hline Lower Bound & & & & 35.22 & 30.82 & 22.68 \\
\hline Upper Bound & & & & 35.86 & 31.66 & 24.46 \\
\hline Maximum Value & & & & 36.40 & 31.92 & 24.86 \\
\hline Minimum Value & & & & 34.77 & 29.73 & 20.51 \\
\hline Range of Values & & & & 1.63 & 2.19 & 4.35 \\
\hline USRP2 & WBX & 1563 & & 32.19 & 24.43 & 20.54 \\
\hline USRPN210 & SBX & F44FFD & F4246A & 32.9 & 32.04 & 26.67 \\
\hline
\end{tabular}


4.1.1.1 Main Results/Observations from 1-dBCP Test

I. At $400 \mathrm{MHz}$ :

- The average value of difference is $35.54 \mathrm{~dB} \rightarrow$ Correction Factor

- The Standard Deviation from this Correction factor amongst the USRPs is $0.52 \mathrm{~dB}$

- This correction factor can be said to lie between $35.54 \pm 0.32=(35.22,35.86) 95$ times out of 100 trials like this of 10 samples.

II. At $900 \mathrm{MHz}$ :

- The average value of difference is $31.24 \mathrm{~dB} \rightarrow$ Correction Factor

- The Standard Deviation from this Correction factor amongst the USRPs is $0.68 \mathrm{~dB}$

- This correction factor can be said to lie between $31.24 \pm 0.42=(30.82,31.66) 95$ times out of 100 trials like this of 10 samples.

III. At $1.800 \mathrm{MHz}$ :

- The average value of difference is $23.57 \mathrm{~dB} \rightarrow$ Correction Factor

- The Standard Deviation from this Correction factor amongst the USRPs is $1.44 \mathrm{~dB}$

- This correction factor can be said to lie between $23.57 \pm 0.89=(22.68,24.46) 95$ times out of 100 trials like this of 10 samples. 


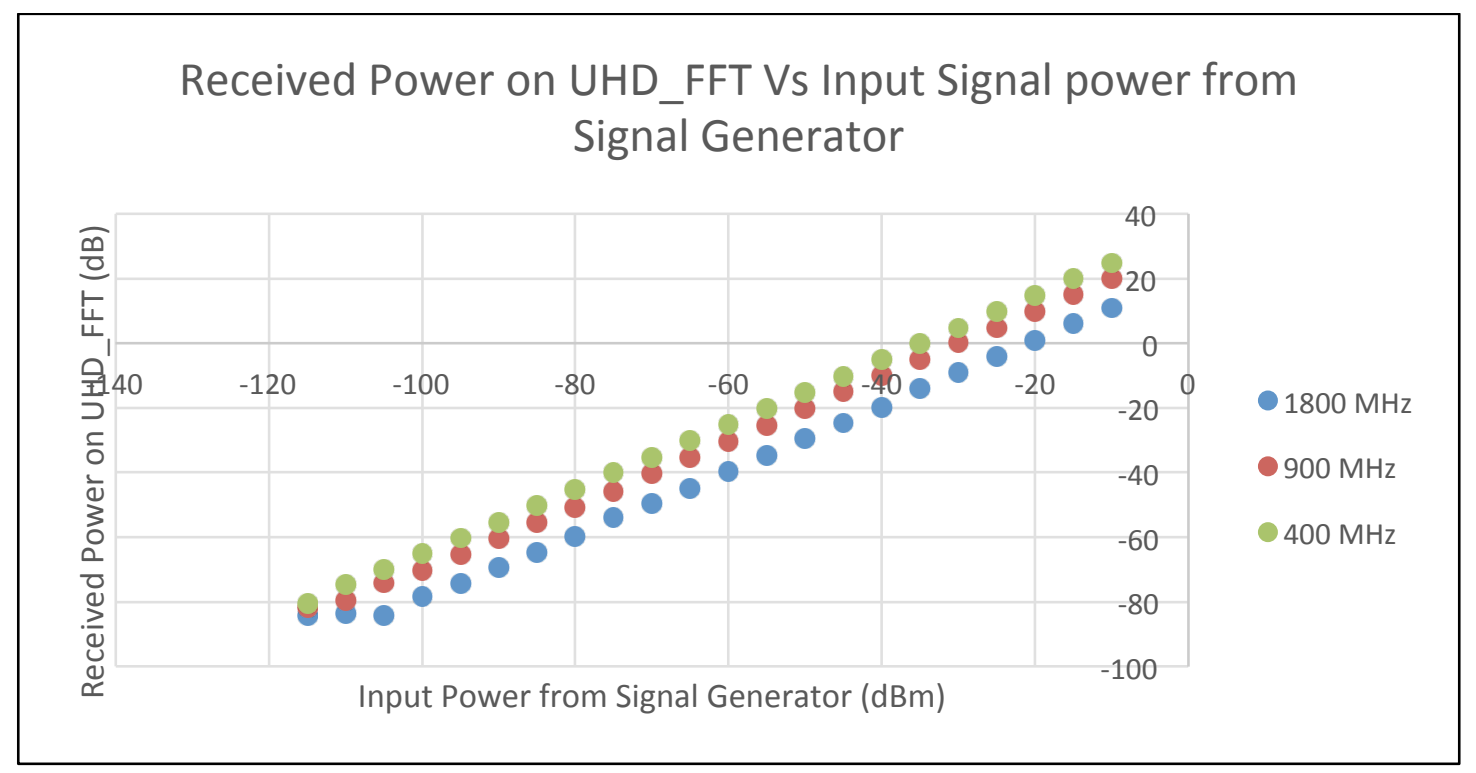

Figure 4.2 Received Power Vs Input Signal Power (USRPN210-WBX DB)

\subsubsection{Received Power Stability Test Procedure}

1. Connect USRPN210 to HOST PC using the gigabit Ethernet cable (blue in the picture)

2. Connect the AGILENT SIGNAL GENERATOR Power Outlet to USRP N210 RX port (RF2) as input via coax cable. The power is first set $-30 \mathrm{dBm}$ and frequency at $50 \mathrm{MHz}$ (lowest frequency of operation of WBX board)

3. Executing the UHD_FFT.grc flow graph set at the $50 \mathrm{MHz}$ and the power is noted generates the received spectrum plot.

4. The frequency is varied in steps of $50 \mathrm{MHz}$ until reaching $2.2 \mathrm{GHz}$ for the same input power and the power on the received spectrum is noted at each step.

5. The power is then set to $-10 \mathrm{dBm}$ and $-60 \mathrm{dBm}$ and the steps from $1-4$ are repeated.

6. The measurements are noted for $-10 \mathrm{dBm},-30 \mathrm{dBm}$ and $-60 \mathrm{dBm}$ for the entire bandwidth of operation of WBX boards for all the USRPS. 
7. From the $3^{\text {rd }}$ USRP the frequency is varied in steps of $100 \mathrm{MHz}$ and the received power on UHD_FFT.grc is noted again for each frequency.

8. This experiment was also repeated for a USRP2 with WBX board and USRPN210 with SBX board.

9. The readings (table 4.2) show that USRPN210 with WBX show steady trend of decrease in output power over the entire frequency range (in steps of 2-4 dB over every $500 \mathrm{MHz}$ ) and so does the SBX board. The difference in the output power for a Daughterboard amongst the 3 input power levels is $\sim 20 \mathrm{~dB}$ and $30 \mathrm{~dB}$ as expected. This helps to imply that raise in input produces a proportional raise in output.

Table 4.2 Readings for Received Power Stability Test

\begin{tabular}{|c|c|c|c|}
\hline \multirow{2}{*}{ USRP Type } & \multirow{2}{*}{ Frequency (MHz) } & \multicolumn{2}{|c|}{$\begin{array}{l}\text { Change in Pout between the } 3 \text { power } \\
\text { levels for frequency in steps of } 500 \mathrm{MHz} \\
\text { (dB) }\end{array}$} \\
\hline & & $\begin{array}{l}-10 \mathrm{dBm} \text { level to - } \\
30 \mathrm{dBm} \text { level } \\
\text { change }\end{array}$ & $\begin{array}{l}-30 \mathrm{dBm} \text { level to - } \\
60 \mathrm{dBm} \text { level } \\
\text { change }\end{array}$ \\
\hline & & & \\
\hline \multirow{4}{*}{$\begin{array}{l}\text { USRPN210 + } \\
\text { WBX }\end{array}$} & 500 & 19.83 & 30.18 \\
\hline & 1000 & 19.87 & 30.04 \\
\hline & 1500 & 20.12 & 30.14 \\
\hline & 2000 & 19.74 & 30.10 \\
\hline \multirow{4}{*}{$\begin{array}{l}\text { USRPN210 + } \\
\text { SBX }\end{array}$} & 500 & 20.00 & 29.89 \\
\hline & 1000 & 20.10 & 30.05 \\
\hline & 1500 & -29.05 & 30.27 \\
\hline & 2000 & -21.22 & 29.39 \\
\hline
\end{tabular}




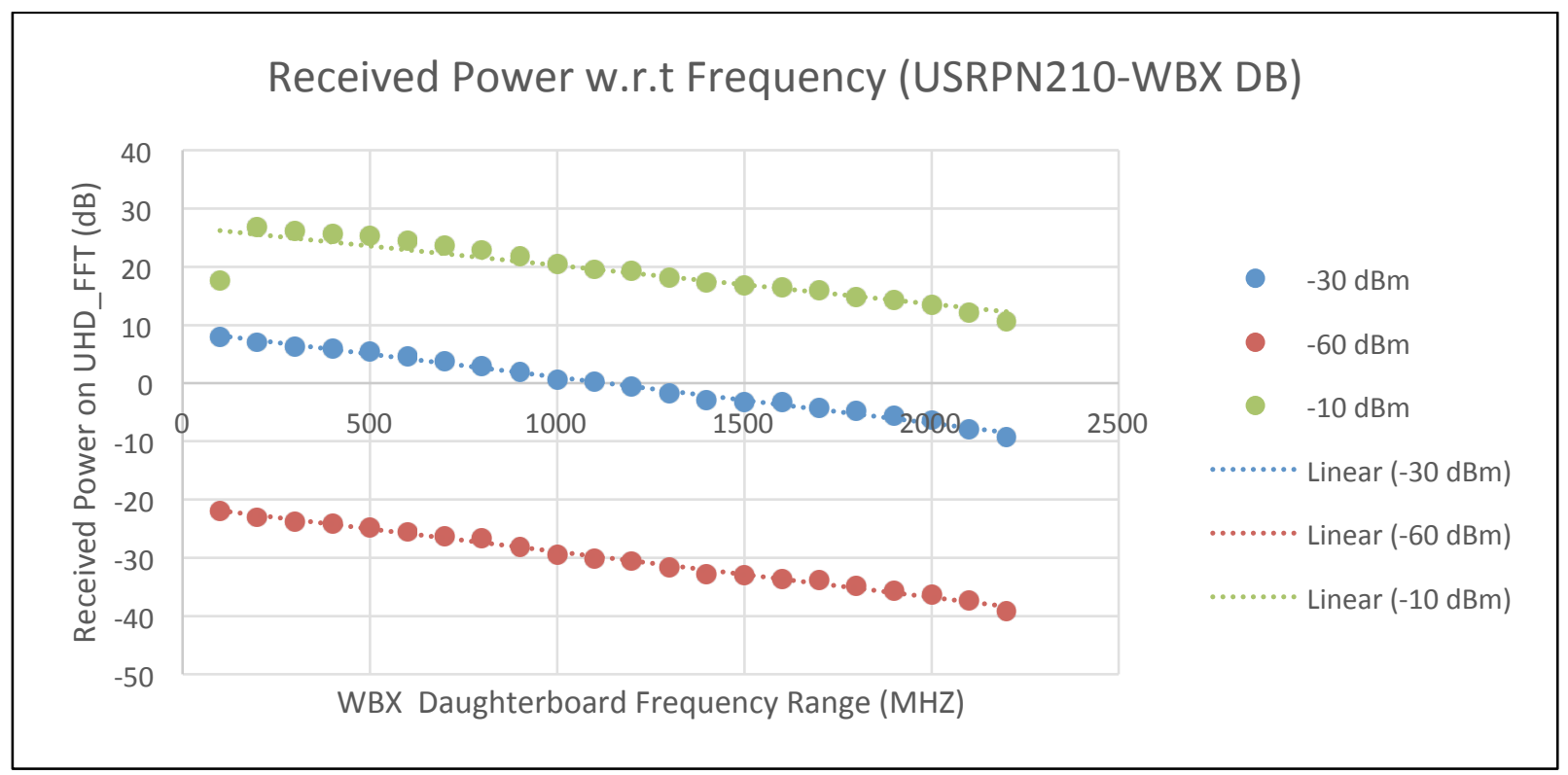

Figure 4.3 Received Power Vs Frequency (USRPN210-WBX DB)

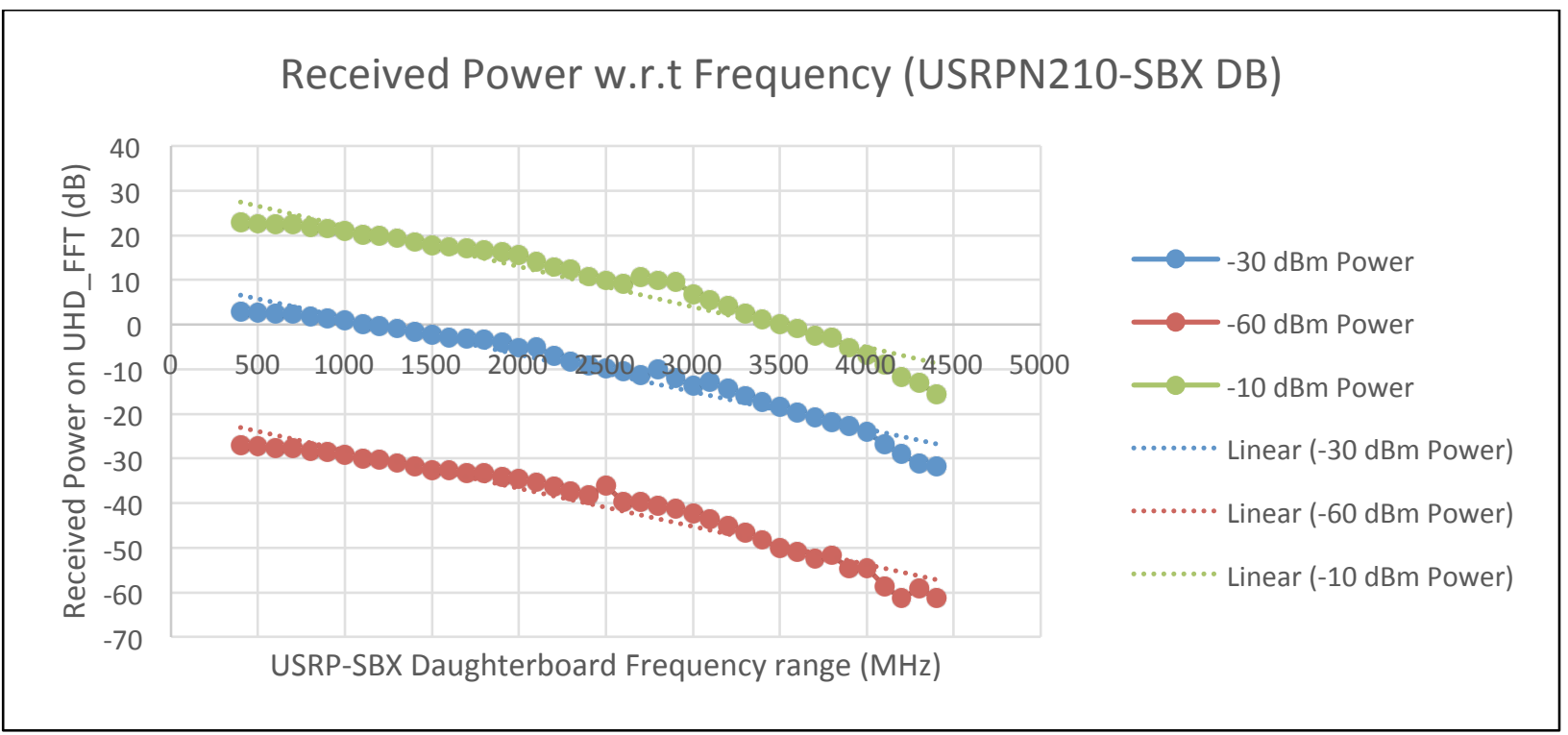

Figure 4.4 Received Power Vs Frequency (USRPN210-SBX DB) 


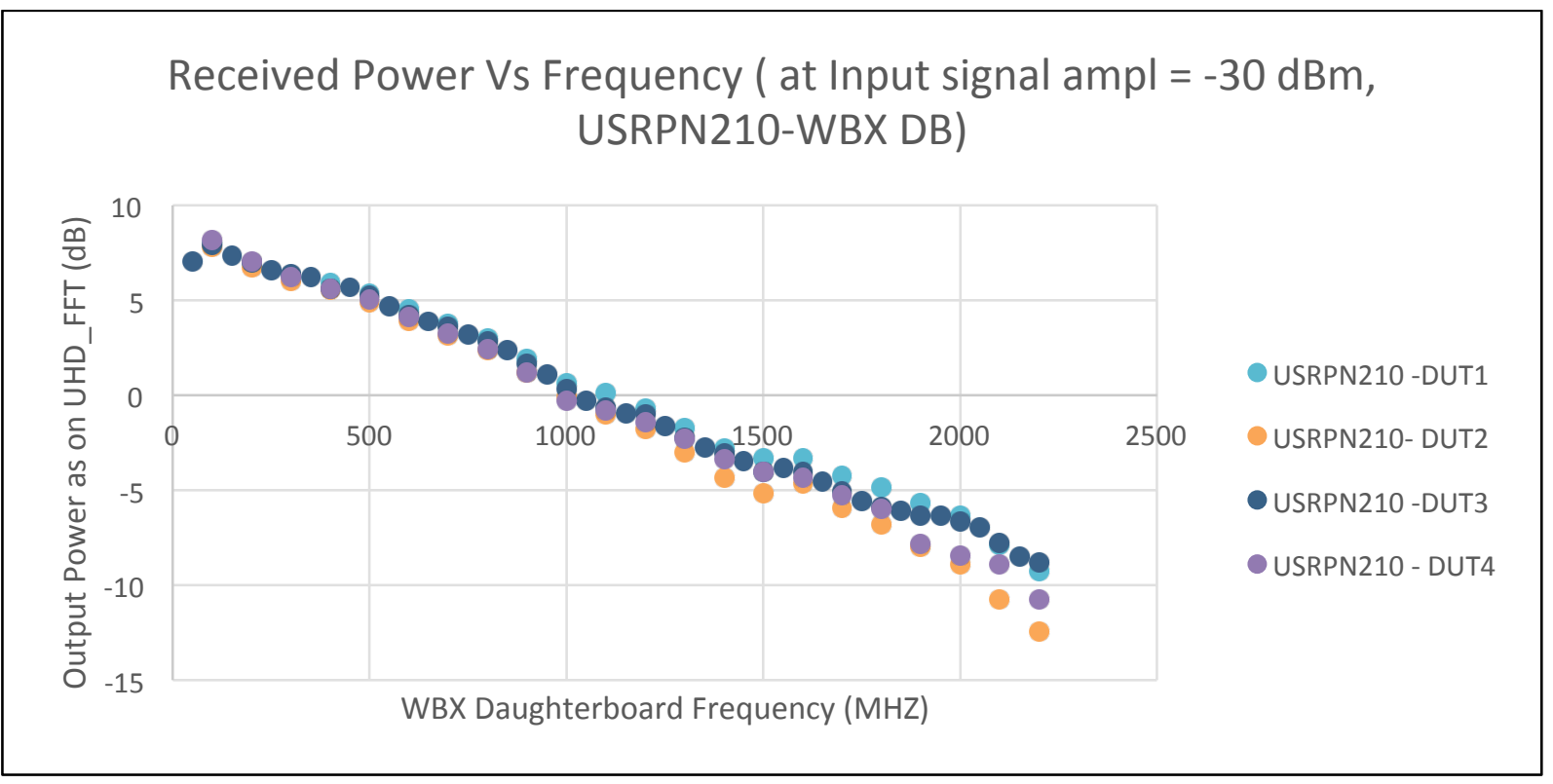

Figure 4.5 Received Power Variation with Frequency @ -30 dBm Input Power

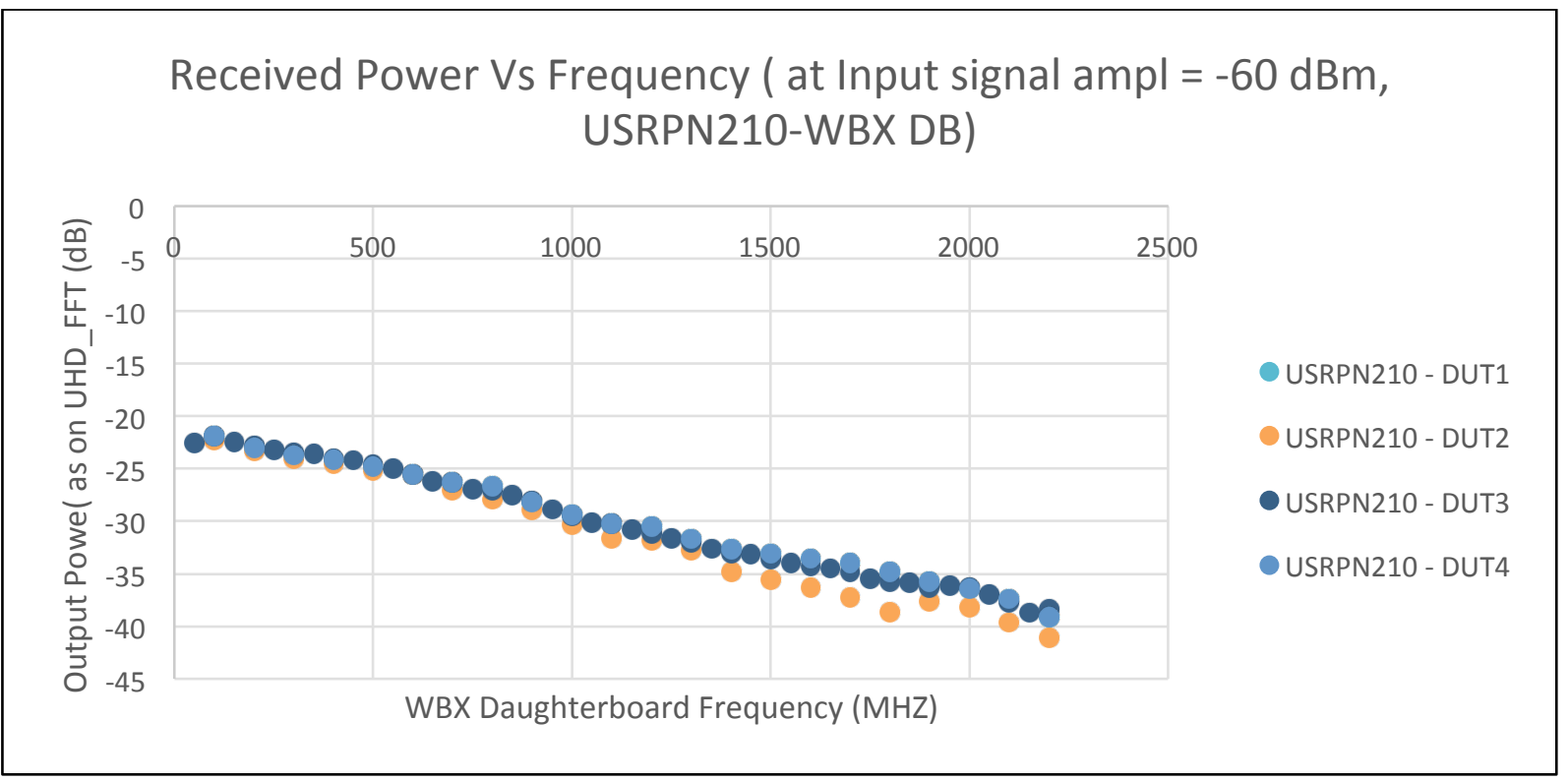

Figure 4.6 Received Power Variation with Frequency @ -60 dBm Input Power 


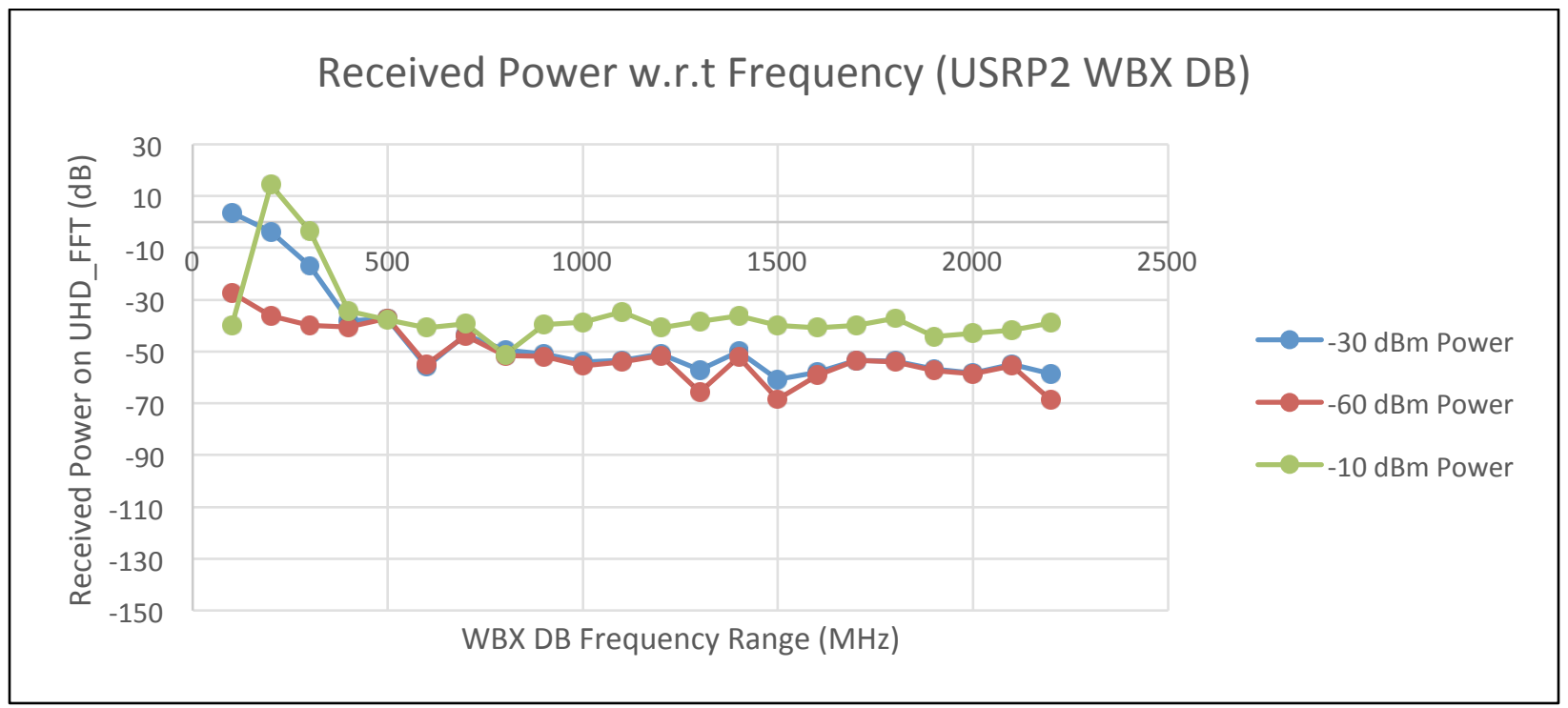

Figure 4.7 Received Power Vs Frequency (USRP2-WBX DB)

\subsubsection{IIP3 Test Procedure}

1. Connect USRPN210 device to HOST PC using the gigabit Ethernet cable (blue in the picture)

2. Two signal generators are used to produce the two fundamental tones. Connect the AGILENT SIGNAL GENERATOR Power Outlet and the HP 8648D SIGNAL GENERATOR to a power combiner (of the required frequency range i.e. $2 \mathrm{GHz}$ )

3. Connect the output of the Power Combiner to the USRP N210 RX port (RF2) as input via a coax cable

4. Set one of the signal generators to frequency of $\mathrm{F} 1=400.1 \mathrm{MHz}$, power at $-70 \mathrm{dBm}$ and another one at F2 $=400.4 \mathrm{MHz}$ since these are the fundamental tones for our test at $400 \mathrm{MHz}$, power at $-70 \mathrm{dBm}$. Power input level of $-70 \mathrm{dBm}$ is chosen as this allows clear signal strength on UHD_FFT above the noise floor without any spurious signals appearing. 
5. The received spectrum plot is generated, by executing the UHD_FFT.grc flow graph set at the desired frequency on the host PC.

6. Tune the center frequency to approximately 400.2 or $400.3 \mathrm{MHz}$ so as to enable view of the fundamental tones and the inter-modulation tones but without any phase noise influence on them.

7. Switch RF ON button on both signal generators. Note the readings on the UHD_FFT plot for F1 and F2.

8. Now disconnect the power combiner output from RF2 port of USRP and connect to the spectrum analyzer. Note down this reading too. This gives the actual value of the power given to USRP device as input (after all the apparatus losses) in $\mathrm{dBm}$. We should be able to see a difference of around $\sim 35 \mathrm{dBm}$ between both sets of readings (correction factor @ 400 $\mathrm{MHz})$.

9. Increase the input signal power on both signal generators in increments of $5 \mathrm{dBm}(-65 \mathrm{dBm},-$ $60 \mathrm{dBm}$ and so on) and note down the readings. Repeat step 8 too for every $10-\mathrm{dBm}$ change.

10. As input signal power is increased, spurious signals will start appearing and the signals occurring at $\mathrm{F}_{\mathrm{IMD} 1}=399.8 \mathrm{MHz}$ and $\mathrm{F}_{\mathrm{IMD} 2}=400.7 \mathrm{MHz}$ are the 3rd order Inter-modulation products.

11. As the signals at $\mathrm{F}_{\mathrm{IMD} 1}$ and $\mathrm{F}_{\mathrm{IMD} 2}$ become detected with clear peaks above the noise floor, start noting down the values of these signals too.

12. To ensure that these signals are actually the inter-modulation products that we are looking for, increase and decrease the input power level on the signal generators and observe the 
trend at these frequencies. If the signals are actually the inter-modulation products they would also follow the trends of input power level i.e. increase and decrease accordingly.

13. Continue this procedure until all the inter-modulation products equal the fundamental tones or until -10 dBm whichever occurs first (Max RX input level for USRP devices).

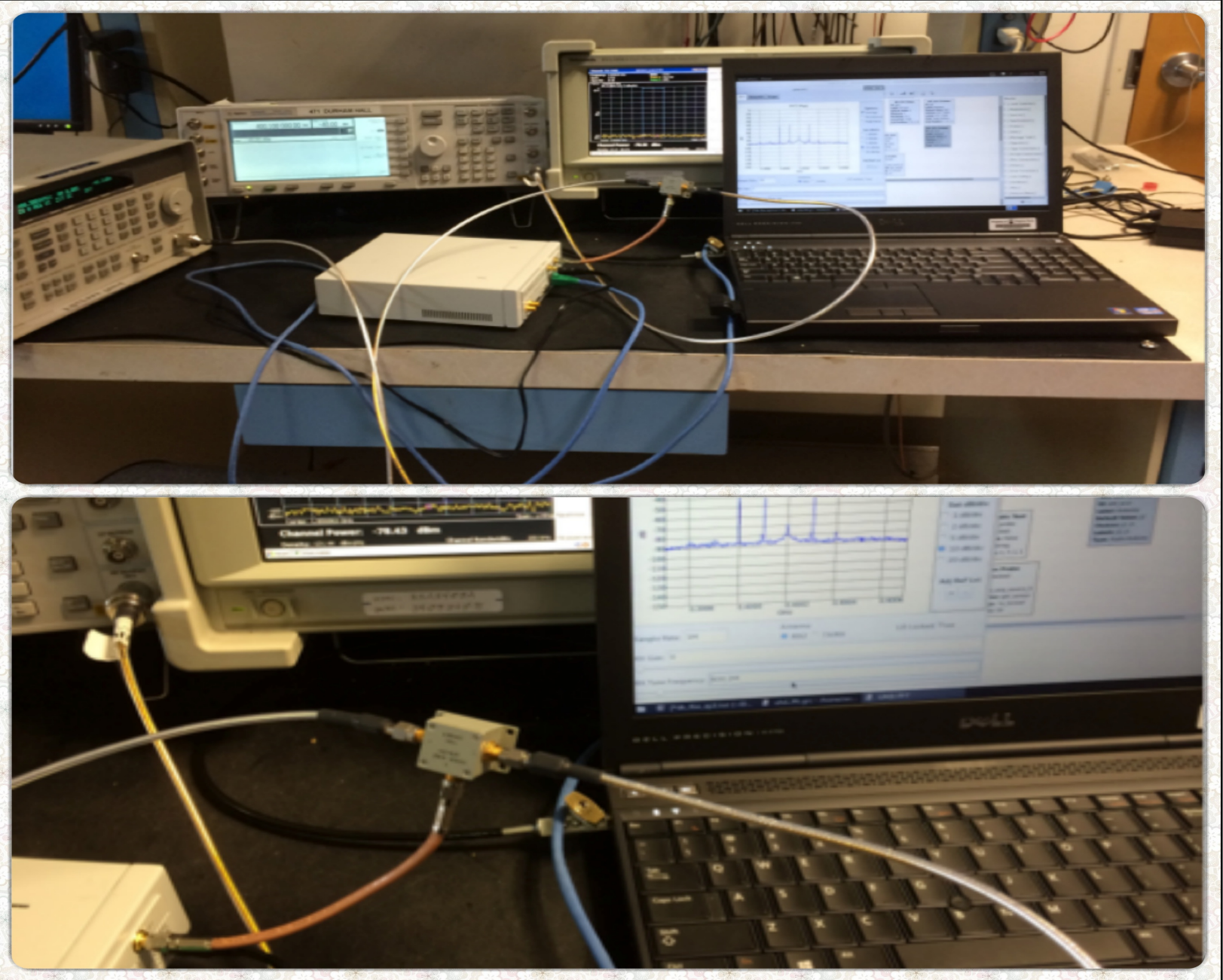

Figure 4.8 Laboratory Setup for IIP3 Test

14. To ensure we get the correct power level of the IMD products too, one of the signal generators is tuned to the power level seen for IMD product and then output of power 
combiner is connected to spectrum analyzer to get the power value in $\mathrm{dBm}$. While this reading is done, the other signal generator must have switched to RF OFF.

15. The readings are tabulated (table 4.3) and then power levels of one fundamental tone and one IMD tone taken from UHD_FFT spectrum are plotted against the input power level. A trend line is added to find out the point of intersection. The intercept on X-axis is the IIP3 value for this device at this frequency.

16. If the readings from the spectrum analyzer are plotted too to get the IIP3 point. This IIP3 point is seen to be closer to expected value than the first one with UHD_FFT readings. If correction factor were applied to the fundamental tone values from UHD_FFT, then we would get close to the expected value again.

17. The same experiment is repeated for 900.MHz for 8 USRPN210+WBX devices (table 4.4). 
Table 4.3 Readings for IIP3 Test@ @400 MHz

\begin{tabular}{|c|c|c|c|c|}
\hline $\begin{array}{c}\text { Sig Gen } 1 \\
\text { readings } \\
@\end{array}$ & $\begin{array}{c}\text { F1 on } \\
\text { UHD_FFT } \\
@\end{array}$ & $\begin{array}{c}\text { IMD1 } \\
\text { UHD_FFT @ }\end{array}$ & $\begin{array}{l}\text { Calibrated F1 i.e. } \\
\text { F1 - (correction + loss) }\end{array}$ & $\begin{array}{l}\text { Calibrated IMD1 i.e. } \\
\text { IMD1 - loss }\end{array}$ \\
\hline $\begin{array}{c}400.1 \mathrm{MHz} \\
\text { (dBm) }\end{array}$ & $\begin{array}{l}400.4 \mathrm{MHz} \\
\text { (dB) }\end{array}$ & $\begin{array}{l}400.7 \mathrm{MHz} \\
\text { (dB) }\end{array}$ & $400.4 \mathrm{MHz}(\mathrm{dBm})$ & $400.7 \mathrm{MHz}(\mathrm{dBm})$ \\
\hline-11.00 & 19.86 & -29.05 & -11.64 & -33.05 \\
\hline-12.00 & 18.95 & -31.98 & -12.56 & -35.98 \\
\hline-13.00 & 18.00 & -34.97 & -13.50 & -38.97 \\
\hline-14.00 & 16.94 & -37.91 & -14.56 & -41.91 \\
\hline-15.00 & 15.94 & -40.77 & -15.56 & -44.77 \\
\hline-16.00 & 15.09 & -43.61 & -16.41 & -47.61 \\
\hline-17.00 & 14.07 & -46.30 & -17.43 & -50.30 \\
\hline-18.00 & 13.03 & -49.32 & -18.47 & -53.32 \\
\hline-19.00 & 12.02 & -52.17 & -19.48 & -56.17 \\
\hline-20.00 & 11.03 & -54.99 & -20.47 & -58.99 \\
\hline-21.00 & 10.08 & -58.32 & -21.42 & -62.32 \\
\hline-22.00 & 9.07 & -60.81 & -22.43 & -64.81 \\
\hline-23.00 & 8.09 & -62.85 & -23.41 & -66.85 \\
\hline-24.00 & 7.02 & -64.93 & -24.48 & -68.93 \\
\hline-25.00 & 5.97 & -69.56 & -25.53 & -73.56 \\
\hline-26.00 & 5.07 & -74.11 & -26.43 & -78.11 \\
\hline-27.00 & 4.06 & -76.00 & -27.44 & -80.00 \\
\hline-28.00 & 3.04 & -74.78 & -28.46 & -78.78 \\
\hline-29.00 & 2.04 & -72.05 & -29.46 & -76.05 \\
\hline-30.00 & 1.05 & -70.07 & -30.45 & -74.07 \\
\hline-31.00 & 0.01 & -69.29 & -31.49 & -73.29 \\
\hline-32.00 & -0.98 & -68.70 & -32.48 & -72.70 \\
\hline-33.00 & -1.98 & -68.81 & -33.48 & -72.81 \\
\hline-34.00 & -2.93 & -71.27 & -34.43 & -75.27 \\
\hline-35.00 & -3.97 & -72.78 & -35.47 & -76.78 \\
\hline-36.00 & -4.94 & -76.81 & -36.44 & -80.81 \\
\hline-37.00 & -5.98 & -85.08 & -37.48 & -89.08 \\
\hline-38.00 & -6.95 & -79.80 & -38.45 & -83.80 \\
\hline-39.00 & -7.96 & -82.64 & -39.46 & -86.64 \\
\hline-40.00 & -8.94 & -82.25 & -40.44 & -86.25 \\
\hline-41.00 & -9.89 & -82.30 & -41.39 & -86.30 \\
\hline-42.00 & -10.91 & -84.66 & -42.41 & -88.66 \\
\hline-43.00 & -11.92 & -86.62 & -43.42 & -90.62 \\
\hline-44.00 & -12.93 & -84.06 & -44.43 & -88.06 \\
\hline-45.00 & -13.93 & -83.63 & -45.43 & -87.63 \\
\hline
\end{tabular}




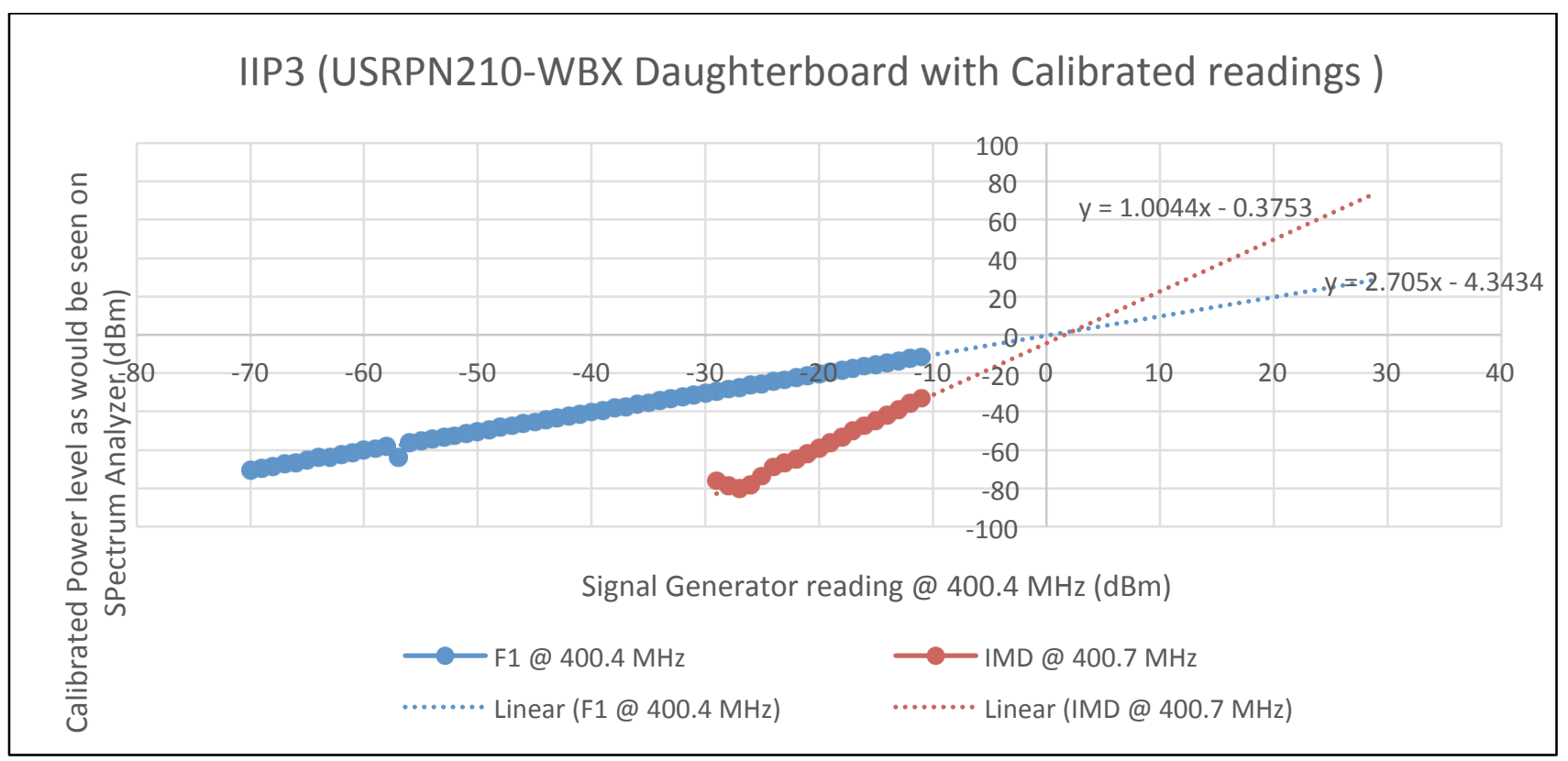

Figure 4.9 IIP3: Calibrated power (USRPN210-WBX DB) @ $400 \mathrm{MHz}$

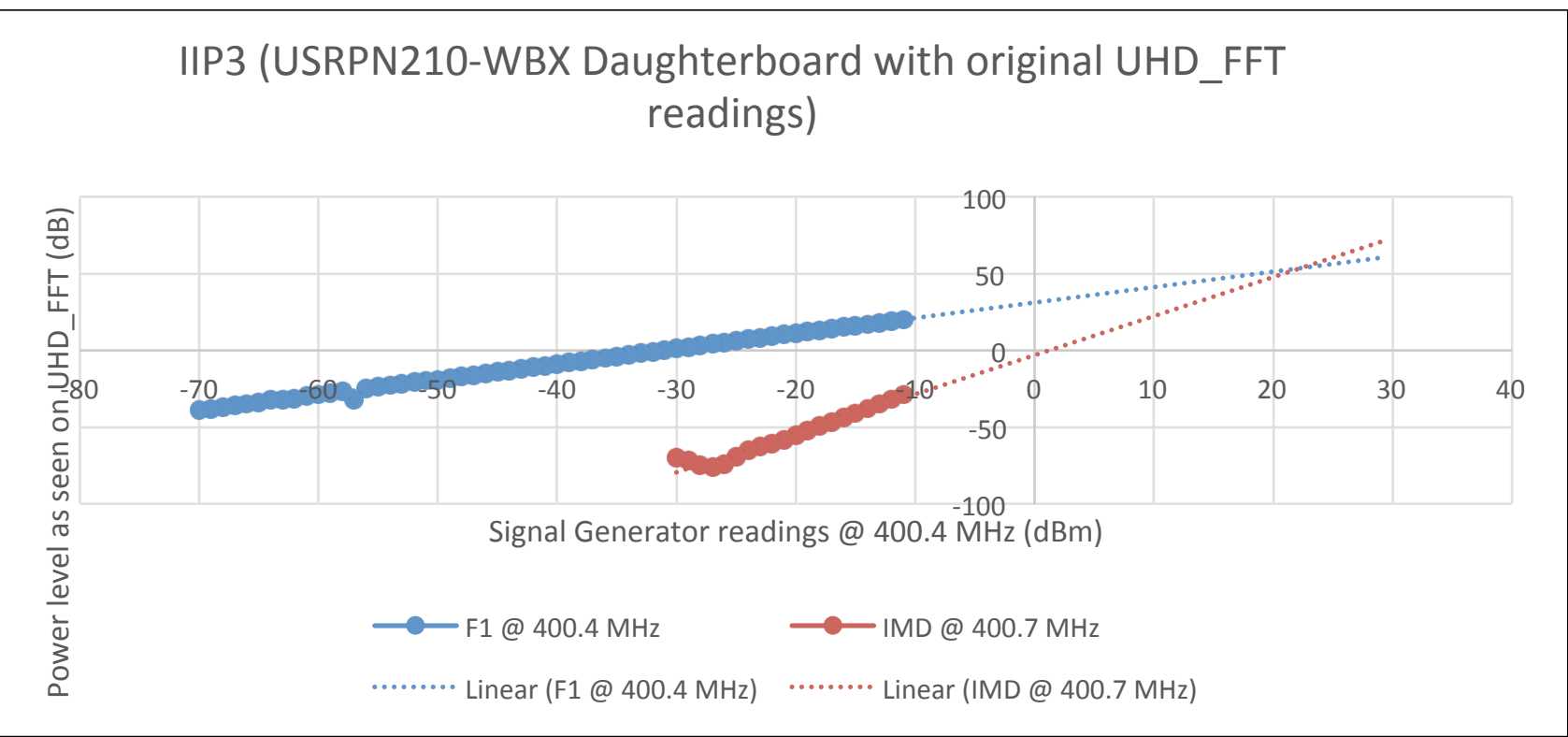

Figure 4.10 IIP3: Un-calibrated power (USRPN210-WBX DB) @ $400 \mathrm{MHz}$ 
Table 4.4 Readings for IIP3 Test @ 900 MHz

\begin{tabular}{|c|c|c|c|c|}
\hline $\begin{array}{l}\text { Sig Gen } 1 \\
\text { reading @ }\end{array}$ & $\begin{array}{c}\text { F1 on } \\
\text { UHD_FFT @ }\end{array}$ & $\begin{array}{c}\text { IMD1 } \\
\text { UHD_FFT @ }\end{array}$ & $\begin{array}{l}\text { Calibrated F1 i.e. } \\
\text { F1- (correction factor + loss) }\end{array}$ & $\begin{array}{l}\text { Calibrated IMD1 i.e. } \\
\text { IMD1 - loss }\end{array}$ \\
\hline $\begin{array}{l}900.1 \mathrm{MHz} \\
(\mathrm{dBm})\end{array}$ & $\begin{array}{l}900.4 \mathrm{MHz} \\
\text { (dB) }\end{array}$ & $\begin{array}{l}900.7 \mathrm{MHz} \\
\text { (dB) }\end{array}$ & $900.4 \mathrm{MHz}(\mathrm{dBm})$ & $900.7 \mathrm{MHz}(\mathrm{dBm})$ \\
\hline-10.00 & 13.98 & -40.06 & -13.22 & -44.06 \\
\hline-11.00 & 12.90 & -42.25 & -14.30 & -46.25 \\
\hline-12.00 & 11.52 & -45.85 & -15.68 & -49.85 \\
\hline-13.00 & 10.99 & -48.60 & -16.21 & -52.60 \\
\hline-14.00 & 9.93 & -52.03 & -17.28 & -56.03 \\
\hline-15.00 & 8.92 & -54.95 & -18.29 & -58.95 \\
\hline-16.00 & 7.74 & -57.74 & -19.46 & -61.74 \\
\hline-17.00 & 6.70 & -60.85 & -20.50 & -64.85 \\
\hline-18.00 & 5.65 & -63.25 & -21.56 & -67.25 \\
\hline-19.00 & 4.66 & -66.20 & -22.54 & -70.20 \\
\hline-20.00 & 3.36 & -70.44 & -23.84 & -74.44 \\
\hline-21.00 & 2.73 & -72.49 & -24.47 & -76.49 \\
\hline-22.00 & 1.69 & -74.94 & -25.51 & -78.94 \\
\hline-23.00 & 0.98 & -76.08 & -26.22 & -80.08 \\
\hline-24.00 & -0.14 & -76.53 & -27.34 & -80.53 \\
\hline-25.00 & -0.86 & -80.93 & -28.06 & -84.93 \\
\hline-26.00 & -1.89 & -72.21 & -29.09 & -76.21 \\
\hline-27.00 & -2.83 & -72.57 & -30.03 & -76.57 \\
\hline-28.00 & -4.05 & -72.60 & -31.25 & -76.60 \\
\hline-29.00 & -5.12 & -74.23 & -32.32 & -78.23 \\
\hline-30.00 & -6.11 & -78.09 & -33.31 & -82.09 \\
\hline-31.00 & -7.07 & -80.97 & -34.27 & -84.97 \\
\hline-32.00 & -7.97 & -86.74 & -35.17 & -90.74 \\
\hline-33.00 & -8.74 & -84.61 & -35.94 & -88.61 \\
\hline-34.00 & -9.69 & -78.07 & -36.89 & -82.07 \\
\hline-35.00 & -11.08 & -81.54 & -38.28 & -85.54 \\
\hline-36.00 & -11.96 & -81.92 & -39.16 & -85.92 \\
\hline-37.00 & -13.02 & -82.96 & -40.22 & -86.96 \\
\hline-38.00 & -14.55 & -82.18 & -41.75 & -86.18 \\
\hline-39.00 & -15.38 & -82.93 & -42.58 & -86.93 \\
\hline-40.00 & -16.38 & -81.45 & -43.58 & -85.45 \\
\hline-41.00 & -17.60 & -85.52 & -44.80 & -89.52 \\
\hline-42.00 & -18.16 & -85.40 & -45.36 & -89.40 \\
\hline-43.00 & -19.22 & -84.24 & -46.42 & -88.24 \\
\hline-44.00 & -20.19 & -85.99 & -47.39 & -89.99 \\
\hline-45.00 & -21.05 & -86.92 & -48.25 & -90.92 \\
\hline
\end{tabular}




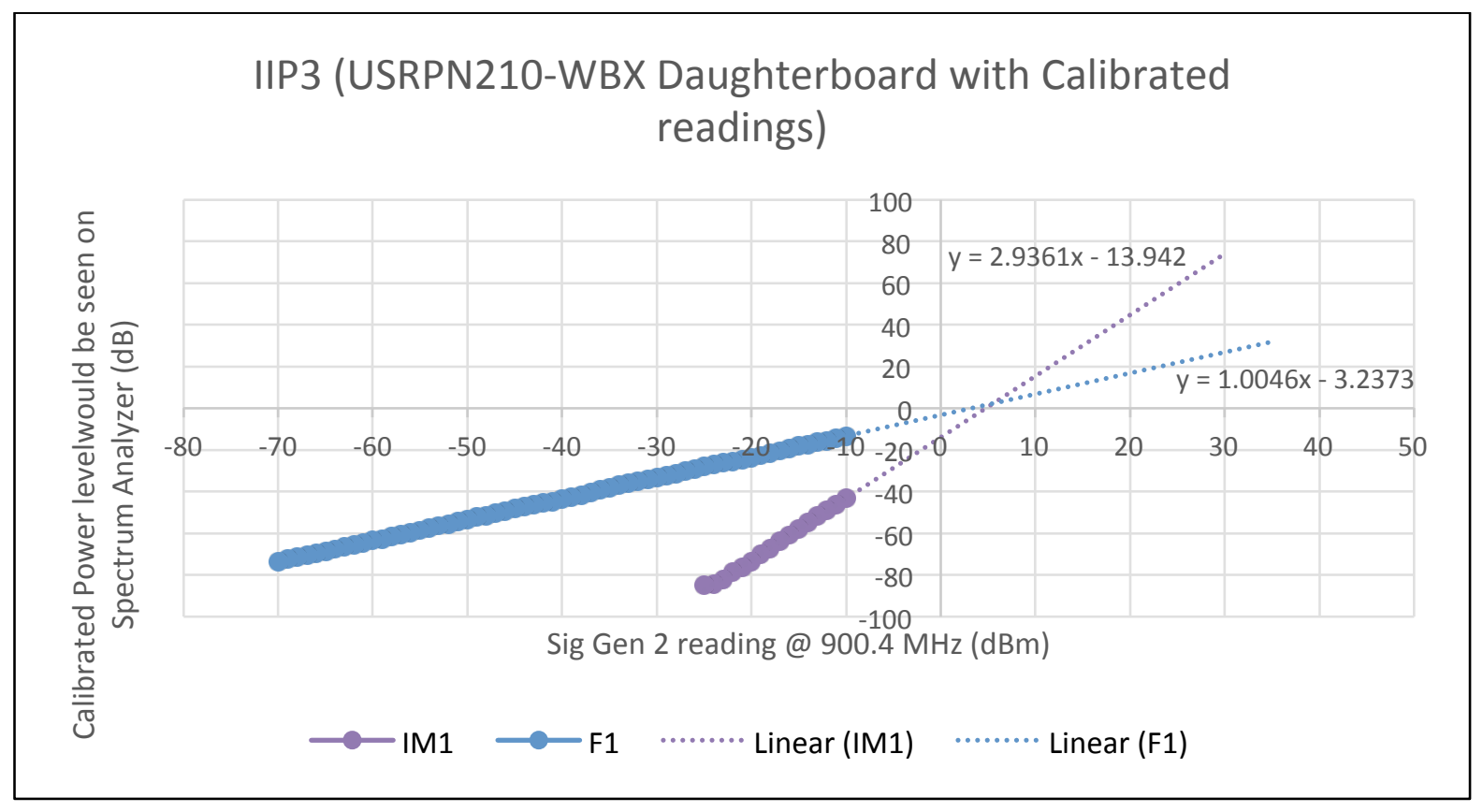

Figure 4.11 IIP3: Calibrated Power (USRPN210-WBX DB) @ $900 \mathrm{MHz}$

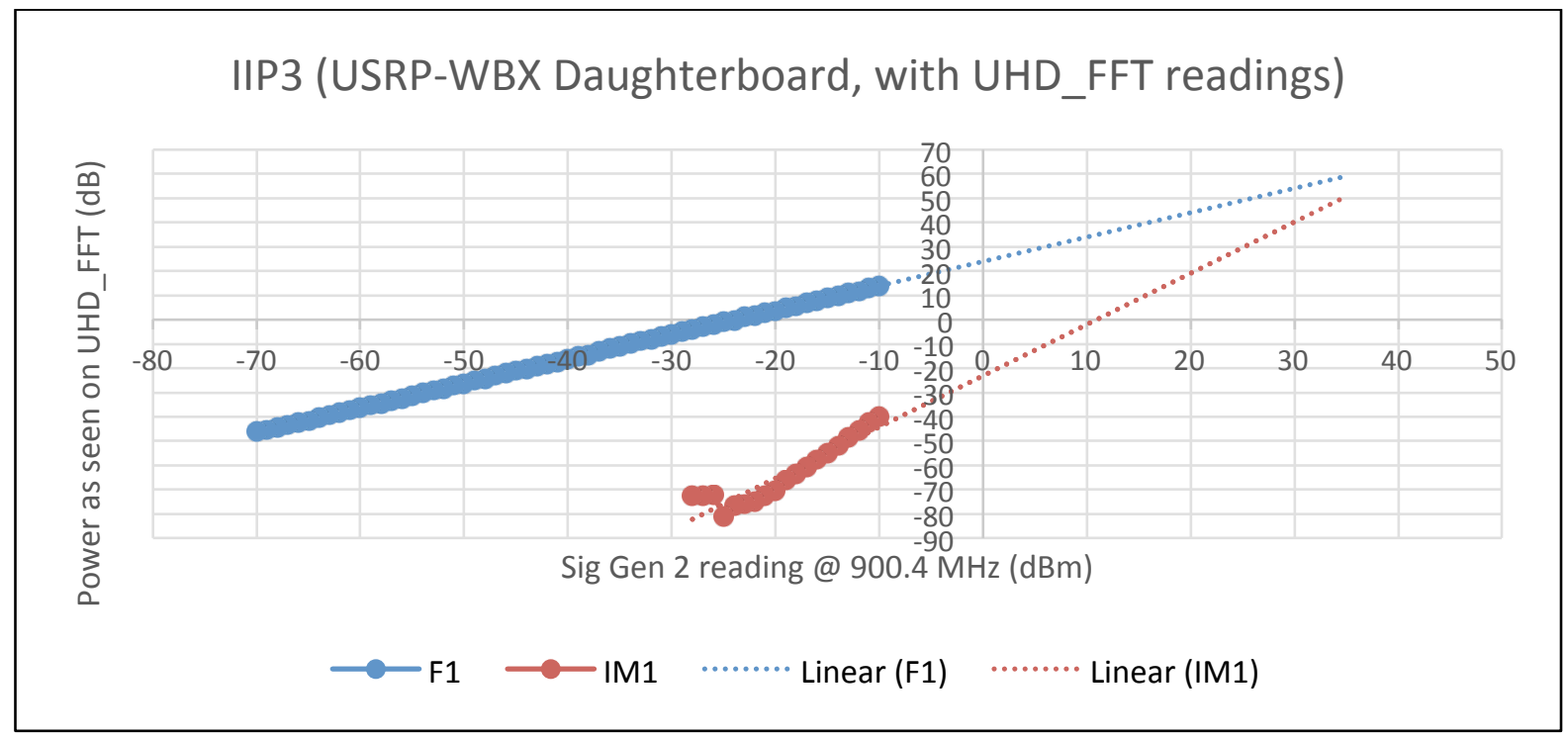

Figure 4.12 IIP3: Un-calibrated Power (USRPN210-WBX DB) @ 900 MHz 
Table 4.5 IIP3 Analytically Calculated @ 400 MHz (USRPN210-WBX DB)

\begin{tabular}{|c|c|c|c|c|c|c|}
\hline \multirow{2}{*}{$\begin{array}{l}\text { Board WBX } \\
\# 361926 \\
\end{array}$} & \multirow{2}{*}{$\begin{array}{c}\begin{array}{c}\text { Center } \\
\text { Frequency }\end{array} \\
400 \mathrm{MHz} \\
\end{array}$} & \multirow{2}{*}{$\begin{array}{l}\text { Signal generator } \\
\text { reading when the } \\
\text { IMD reading is } \\
\text { taken (usually } \\
\text { same on both } \\
\text { signal generators) } \\
-33\end{array}$} & \multicolumn{3}{|c|}{ O/p reading on UHD_FFT } & \multirow{2}{*}{$\begin{array}{c}\text { iip3 }=[(\mathrm{F} 1-\mathrm{IM} 1) / 2+ \\
\mathrm{F} 1-(\text { Correction } \\
\text { factor }+ \\
\text { loss })](\mathrm{dBm})\end{array}$} \\
\hline & & & -1.98 & -2.05 & -68.81 & \\
\hline$\# 361927$ & $400 \mathrm{MHz}$ & -33 & -2.61 & -2.87 & -76.51 & 2.58 \\
\hline \# 361928 & $400 \mathrm{MHz}$ & -34 & -3.19 & -3.85 & -73.53 & -0.18 \\
\hline \# 361929 & $400 \mathrm{MHz}$ & -34 & -5.24 & -5.34 & -77.78 & -0.56 \\
\hline \# 361934 & $400 \mathrm{MHz}$ & -34 & 3.18 & -6.86 & -75.90 & 1.18 \\
\hline \# 361935 & $400 \mathrm{MHz}$ & -37.5 & -5.50 & -6.57 & -76.63 & -2.51 \\
\hline \# 361936 & $400 \mathrm{MHz}$ & -32 & 0.14 & -8.66 & -78.38 & -0.91 \\
\hline \# 361939 & $400 \mathrm{MHz}$ & -29 & -2.53 & -3.04 & -76.66 & 2.53 \\
\hline Average & & & & & & 0.25 \\
\hline Standard Deviation & & & & & & 1.75 \\
\hline $\begin{array}{c}95 \% \text { Confidence Interval } \\
\text { (Margin of Error) }\end{array}$ & & & & & & 1.21 \\
\hline Lower Bound & & & & & & -0.96 \\
\hline Upper Bound & & & & & & 1.46 \\
\hline Maximum Value & & & & & & 2.58 \\
\hline Minimum Value & & & & & & -2.51 \\
\hline Range of Values & & & & & & 5.09 \\
\hline
\end{tabular}

\subsection{TX Characterization}

The below picture depicts the setup used for most of the Transmitter side tests conducted.

The blue cable, which is the gigabit Ethernet cable, connects the host laptop and DUT. The TX (RF1) port is connected to the Spectrum Analyzer (RSA 3408, operating frequency: DC - 6 $\mathrm{GHz}$ ) using coaxial cable of around $500 \mathrm{~cm}$, via an external attenuator at times for preventing 
spectrum Analyzer's DAC from overflowing. The power meter (HP 438A) is used for TX power variation with TX gain test and also for initial calibration with spectrum analyzer readings.

Table 4.6 IIP3 Analytically Calculated @ 900 MHz (USRPN210-WBX)

\begin{tabular}{|c|c|c|c|c|c|c|}
\hline \multirow{2}{*}{$\begin{array}{c}\text { Board WBX } \\
\# 361926\end{array}$} & \multirow{2}{*}{$\begin{array}{c}\begin{array}{c}\text { Center } \\
\text { Frequency }\end{array} \\
900 \mathrm{MHz}\end{array}$} & \multirow{2}{*}{$\begin{array}{l}\text { Signal generator } \\
\text { reading when the } \\
\text { IMD reading is taken } \\
\text { (usually same on both } \\
\text { signal generators) } \\
-23.5\end{array}$} & \multicolumn{3}{|c|}{ O/p reading on UHD_FFT } & \multirow{2}{*}{$\begin{array}{c}\text { iip3 = [(F1- } \\
\text { IM1)/2 + F1 } \\
- \\
\text { (Correction } \\
\text { factor + } \\
\text { loss)](dBm) } \\
2.29\end{array}$} \\
\hline & & & -8.74 & -8.61 & -84.93 & \\
\hline \# 361927 & $900 \mathrm{MHz}$ & -28.5 & -1.88 & -3.05 & -76.29 & 6.95 \\
\hline \# 361928 & $900 \mathrm{MHz}$ & -32.5 & -3.65 & -5.31 & -76.13 & 3.73 \\
\hline \# 361929 & $900 \mathrm{MHz}$ & -29.4 & -1.98 & -2.05 & -68.81 & 4.16 \\
\hline \# 361934 & $900 \mathrm{MHz}$ & -29 & -5.95 & -5.86 & -76.75 & 2.34 \\
\hline \# 361935 & $900 \mathrm{MHz}$ & -31 & -5.44 & -8.05 & -75.59 & -0.18 \\
\hline \# 361936 & $900 \mathrm{MHz}$ & -23 & -2.59 & -2.67 & -76.62 & 7.14 \\
\hline \# 361939 & $900 \mathrm{MHz}$ & -30 & -2.56 & -4.59 & -76.61 & 5.24 \\
\hline Average & & & & & & 3.96 \\
\hline $\begin{array}{l}\text { Standard } \\
\text { Deviation }\end{array}$ & & & & & & 2.49 \\
\hline $\begin{array}{c}95 \% \text { Confidence } \\
\text { Interval (Margin } \\
\text { of Error) }\end{array}$ & & & & & & 1.72 \\
\hline Lower Bound & & & & & & 2.24 \\
\hline Upper Bound & & & & & & 5.68 \\
\hline Maximum Value & & & & & & 7.14 \\
\hline Minimum Value & & & & & & -0.18 \\
\hline Range of Values & & & & & & 7.31 \\
\hline
\end{tabular}




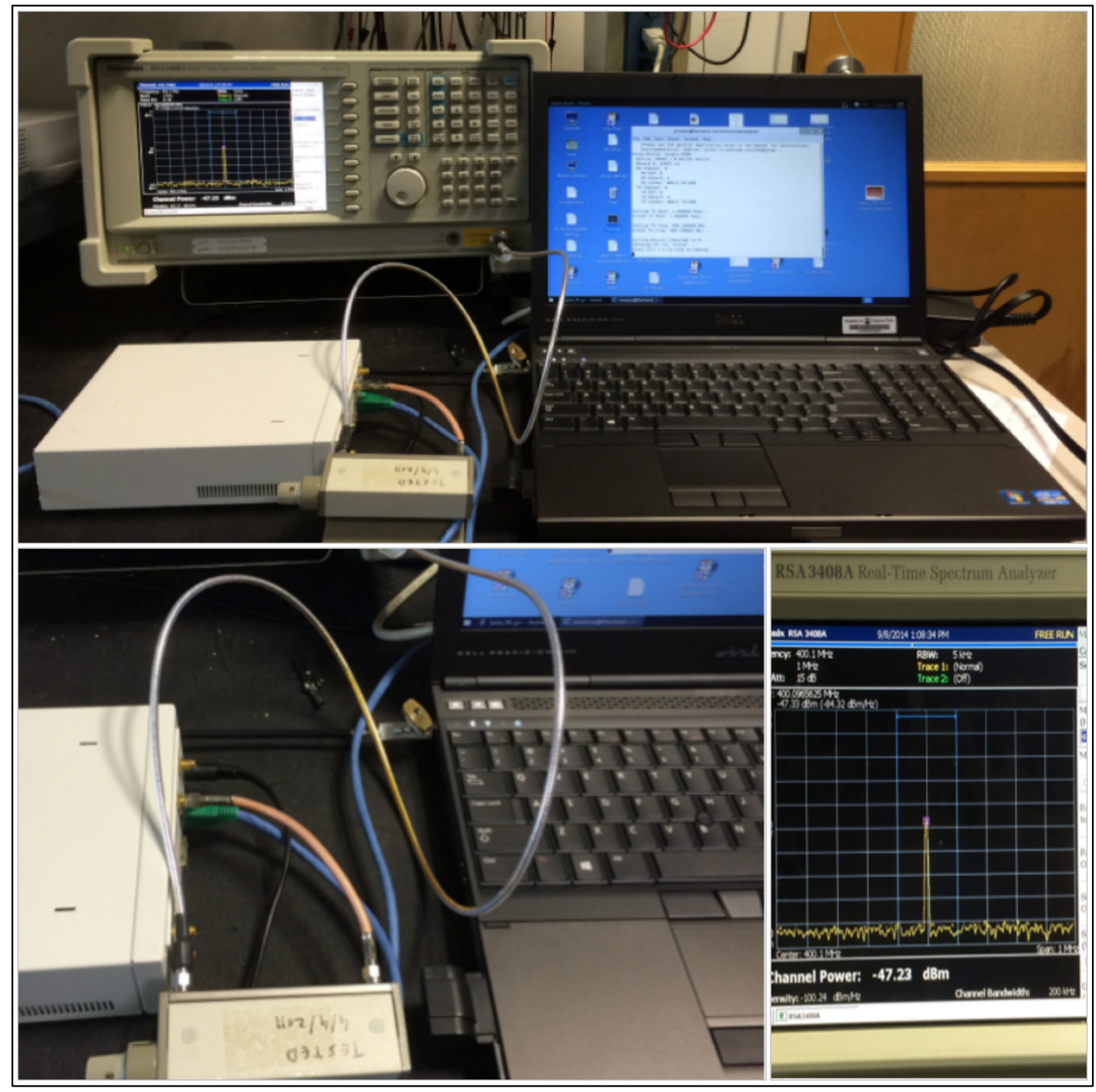

Figure 4.13 Laboratory Setup for TX Frequency and TX Power Stability Test

The black cable is the power cable for the USRPN210 providing a DC voltage of $6 \mathrm{~V}$. As mentioned earlier, the Tektronix Spectrum Analyzer is used for output interface as the USRP device behaves at the transmitter here. The UHD_SIGGEN.py utility provided in the UHD package is used as the signal generator, which produces the digital samples at the required 
frequency. The UHD_SIGGEN.py has the capability to produce single tone and two-tone Sine wave signals.

\subsubsection{Transmitted Signal Frequency Stability Test Procedure}

1. Connect the USRPN210 to the HOST PC using gigabit Ethernet cable (blue in the picture)

2. Connect the USRP N210 TX port (RF1) to the input of Spectrum Analyzer via an adjustable attenuator to provide $30 \mathrm{~dB}$ attenuation before the input is given to spectrum analyzer to prevent any damages.

3. The spectrum analyzer is set on Real time Sample and Hold mode. The RBW set to 1 $\mathrm{MHz}$, the acquiring time gets set to $51.2 \mathrm{~s}$ while the processing time is $51.2 \mathrm{sec}$. frequency.

4. Choose the carrier frequency measurement that can be explicitly to get the real time measurement of the RF carrier frequency on the spectrum Analyzer.

5. The UHD_SIGGEN.py is executed with the following input parameters: -Amplitude $=1$, -s = 1e6, -f 400e6, -x 100e3 -gain 0

6. A sine wave signal is produced at $400.1 \mathrm{MHz}$ and hence the center frequency on the spectrum analyzer is also set as $400.1 \mathrm{MHz}$.

7. After approximately 2 mins, the signal would be visible on the spectrum analyzers screen. The carrier frequency measure in real time would be updated just below the spectrum on the screen. Note this value down. 
8. After approximately 2 mins again the value would be updated. Note down the readings at the interval of every 2 mins for the first 1-hour, then at an interval of 10 mins for next hour.

Table 4.7 Readings for Transmitted Frequency Stability Test

\begin{tabular}{|c|c|c|c|c|c|c|}
\hline \multirow[t]{2}{*}{ USRP Type } & \multicolumn{3}{|c|}{$\begin{array}{l}\text { Average Frequency Difference or } \\
\text { offset from the expected Frequency } \\
\text { i.e. Carrier frequency on spectrum } \\
\text { analyzer - Expected Frequency (over a } \\
\text { period of } 1 \text { hour) (MHz) }\end{array}$} & \multicolumn{3}{|c|}{$\begin{array}{l}\text { Average Frequency Deviation in ppm } \\
\text { i.e.Avg Freq. Dev / Expected Frequency } \\
* 10^{\wedge} 6(\mathrm{ppm})\end{array}$} \\
\hline & $400 \mathrm{MHz}$ & $900 \mathrm{MHz}$ & $1800 \mathrm{MHz}$ & $400 \mathrm{MHz}$ & $900 \mathrm{MHz}$ & $1800 \mathrm{MHz}$ \\
\hline \multicolumn{7}{|l|}{$\begin{array}{c}\text { USRPN210(s) with WBX } \\
\text { daughter boards }\end{array}$} \\
\hline VT361926 & $-3.34 \mathrm{E}-04$ & $-6.90 \mathrm{E}-04$ & $-1.42 \mathrm{E}-03$ & -0.83 & -0.77 & -0.79 \\
\hline VT361927 & $-2.62 E-04$ & $3.00 \mathrm{E}-04$ & $-1.15 \mathrm{E}-03$ & -0.66 & 0.33 & -0.64 \\
\hline VT361928 & $-2.61 E-04$ & $-5.81 E-04$ & $-1.49 \mathrm{E}-03$ & -0.65 & -0.64 & -0.83 \\
\hline VT361929 & $3.13 \mathrm{E}-05$ & $1.11 \mathrm{E}-04$ & 4.73E-04 & 0.08 & 0.12 & 0.26 \\
\hline VT361930 & $1.03 E-05$ & $-5.19 E-04$ & $-9.46 \mathrm{E}-04$ & 0.03 & -0.58 & -0.53 \\
\hline VT361931 & $6.28 \mathrm{E}-05$ & $3.04 \mathrm{E}-04$ & $1.01 \mathrm{E}-03$ & 0.16 & 0.34 & 0.56 \\
\hline VT361932 & $2.70 \mathrm{E}-04$ & $1.73 \mathrm{E}-04$ & $5.21 \mathrm{E}-04$ & 0.67 & 0.19 & 0.29 \\
\hline VT361933 & $-7.15 E-04$ & $-5.46 \mathrm{E}-05$ & $-8.24 \mathrm{E}-04$ & -1.79 & -0.06 & -0.46 \\
\hline VT361936 & $-2.63 \mathrm{E}-04$ & $-3.27 \mathrm{E}-04$ & $-8.64 \mathrm{E}-04$ & -0.66 & -0.36 & -0.48 \\
\hline VT361937 & $1.87 \mathrm{E}-04$ & $5.11 \mathrm{E}-04$ & $-1.31 \mathrm{E}-04$ & 0.47 & 0.57 & -0.07 \\
\hline Mean Frequency Offset & $-1.27 \mathrm{E}-04$ & $-7.73 \mathrm{E}-05$ & $-4.82 \mathrm{E}-04$ & -0.32 & -0.09 & -0.27 \\
\hline Standard Deviation & $2.94 \mathrm{E}-04$ & $4.24 \mathrm{E}-04$ & 8.87E-04 & 0.74 & 0.47 & 0.49 \\
\hline $\begin{array}{c}95 \% \text { confidence Interval } \\
\text { (Margin of error) }\end{array}$ & $1.82 \mathrm{E}-04$ & $2.63 \mathrm{E}-04$ & $5.50 \mathrm{E}-04$ & 0.46 & 0.29 & 0.31 \\
\hline RMS off-set & 3.07E-04 & 4.10E-04 & $9.70 \mathrm{E}-04$ & 7.67E-01 & 4.55E-01 & $5.40 \mathrm{E}-01$ \\
\hline Lower Bound & $-3.10 \mathrm{E}-04$ & $-3.40 \mathrm{E}-04$ & $-1.03 E-03$ & -0.77 & -0.38 & -0.57 \\
\hline Upper Bound & $5.50 \mathrm{E}-05$ & $1.86 \mathrm{E}-04$ & $6.79 \mathrm{E}-05$ & 0.14 & 0.21 & 0.04 \\
\hline Maximum Value & $2.70 \mathrm{E}-04$ & $5.11 \mathrm{E}-04$ & $1.01 \mathrm{E}-03$ & 0.67 & 0.57 & 0.56 \\
\hline Minimum Value & $-7.15 E-04$ & $-6.90 \mathrm{E}-04$ & $-1.49 \mathrm{E}-03$ & -1.79 & -0.77 & -0.83 \\
\hline Range of Values & $9.85 \mathrm{E}-04$ & $1.20 \mathrm{E}-03$ & $2.50 \mathrm{E}-03$ & 2.46 & 1.34 & 1.39 \\
\hline USRP2-WBX & $3.22 \mathrm{E}-03$ & $8.53 \mathrm{E}-03$ & $1.70 \mathrm{E}-05$ & 8.05 & 9.47 & 0.01 \\
\hline USRPN210-SBX & $2.12 \mathrm{E}-03$ & $6.91 \mathrm{E}-04$ & $-1.36 \mathrm{E}-03$ & 5.31 & 0.77 & -0.76 \\
\hline
\end{tabular}


9. Repeat the steps from 5 to 8 for $900 \mathrm{MHz}$ and $1.800 \mathrm{MHz}$ in line with other tests. The UHD_SIGGEN parameters will change accordingly (-f $=900 \mathrm{e} 6$ and $-\mathrm{f}=1800 \mathrm{e} 6$ resp.)

10. The readings have been tabulated in table 4.7 for the 10 devices at the 3 different frequencies. It can be seen that @ $900 \mathrm{MHz}$ the deviation seem to be around 2.01 ppm while@400 MHz and@1.8 GHz the deviations are minimal.

11. This experiment was also repeated for a USRP2 with WBX board and USRPN210 with SBX board.

12. The readings show that the USRPN210 with SBX has a higher deviation of 5.3 ppm @ $400 \mathrm{MHz}$, while negligible deviations @, $900 \mathrm{MHz}$ and $1.8 \mathrm{GHz}$.

13. As for USRP2, a frequency deviation of 8.05 ppm and 9.47 ppm is seen @ $400 \mathrm{MHz}$ and $900 \mathrm{MHz}$ while at $1.8 \mathrm{GHz}$ the frequency deviation is negligible at $0.01 \mathrm{ppm}$.

\subsubsection{Main Results/Observations from TX Frequency Stability Test}

I. At $400 \mathrm{MHz}$ :

- The average value of difference is $-0.32 \mathrm{ppm} \rightarrow$ Correction Factor

- The Standard Deviation from this Correction factor amongst the USRPs is $0.74 \mathrm{ppm}$

- This deviation factor can be said to lie between $-0.32 \pm 0.46=(-0.77,+0.14)$ ppm 95 times out of 100 trials like this of 10 samples.

II. At $900 \mathrm{MHz}$ :

- The average value of difference is $-0.09 \mathrm{ppm} \rightarrow$ Correction Factor

- The Standard Deviation from this Correction factor amongst the USRPs is $0.47 \mathrm{ppm}$ 
- This deviation factor can be said to lie between $-0.09 \pm 0.29=(-0.38,0.21)$ ppm 95 times out of 100 trials like this of 10 samples.

III. At $1.800 \mathrm{MHz}$ :

- The average value of difference is $-0.27 \mathrm{ppm} \rightarrow$ Correction Factor

- The Standard Deviation from this Correction factor amongst the USRPs is $0.49 \mathrm{ppm}$

- This deviation factor can be said to lie between $-0.27 \pm 0.31=(-0.57,0.04)$ ppm 95 times out of 100 trials like this of 10 samples.

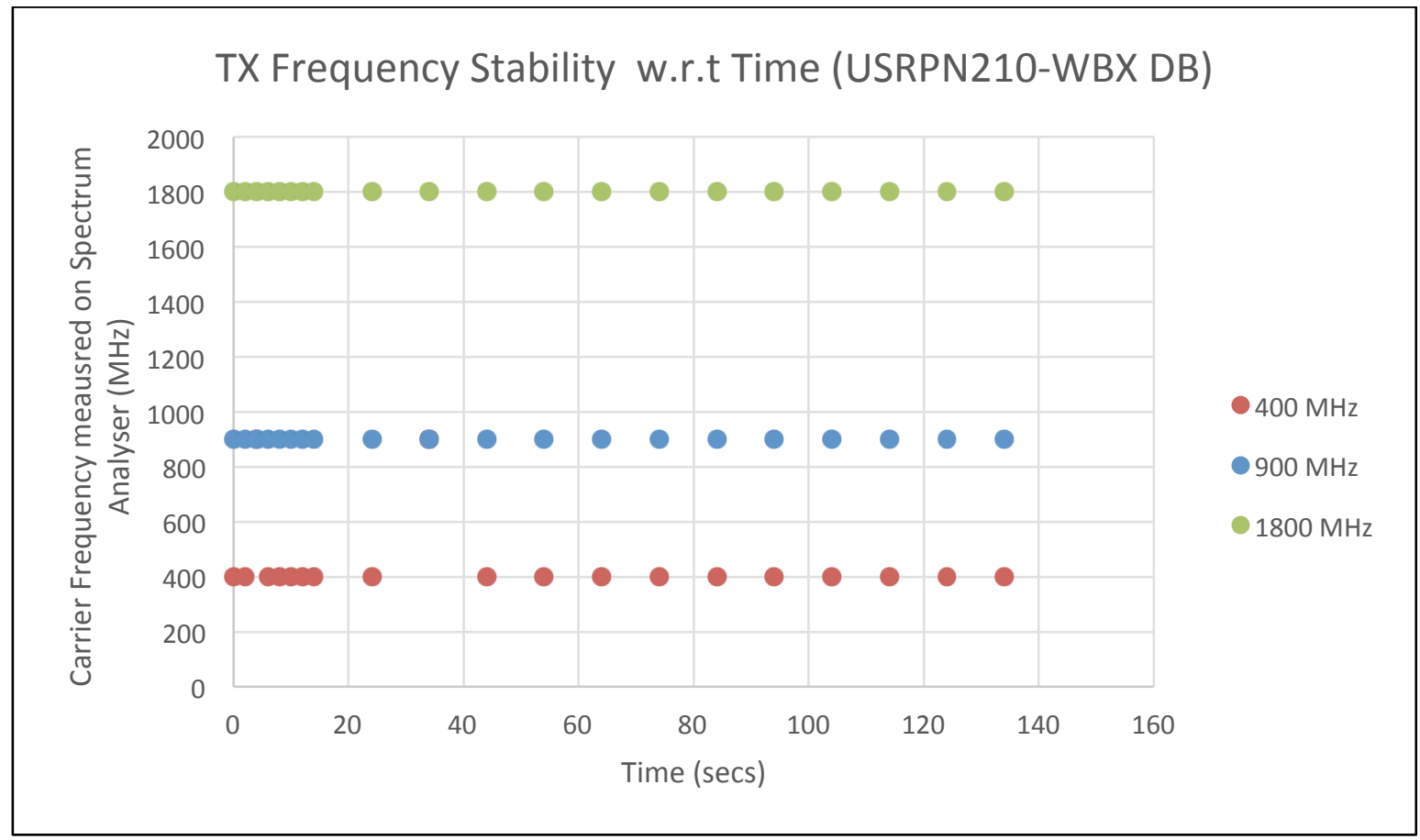

Figure 4.14 Transmitted Frequency Stability Test (USRPN210-WBX DB) 


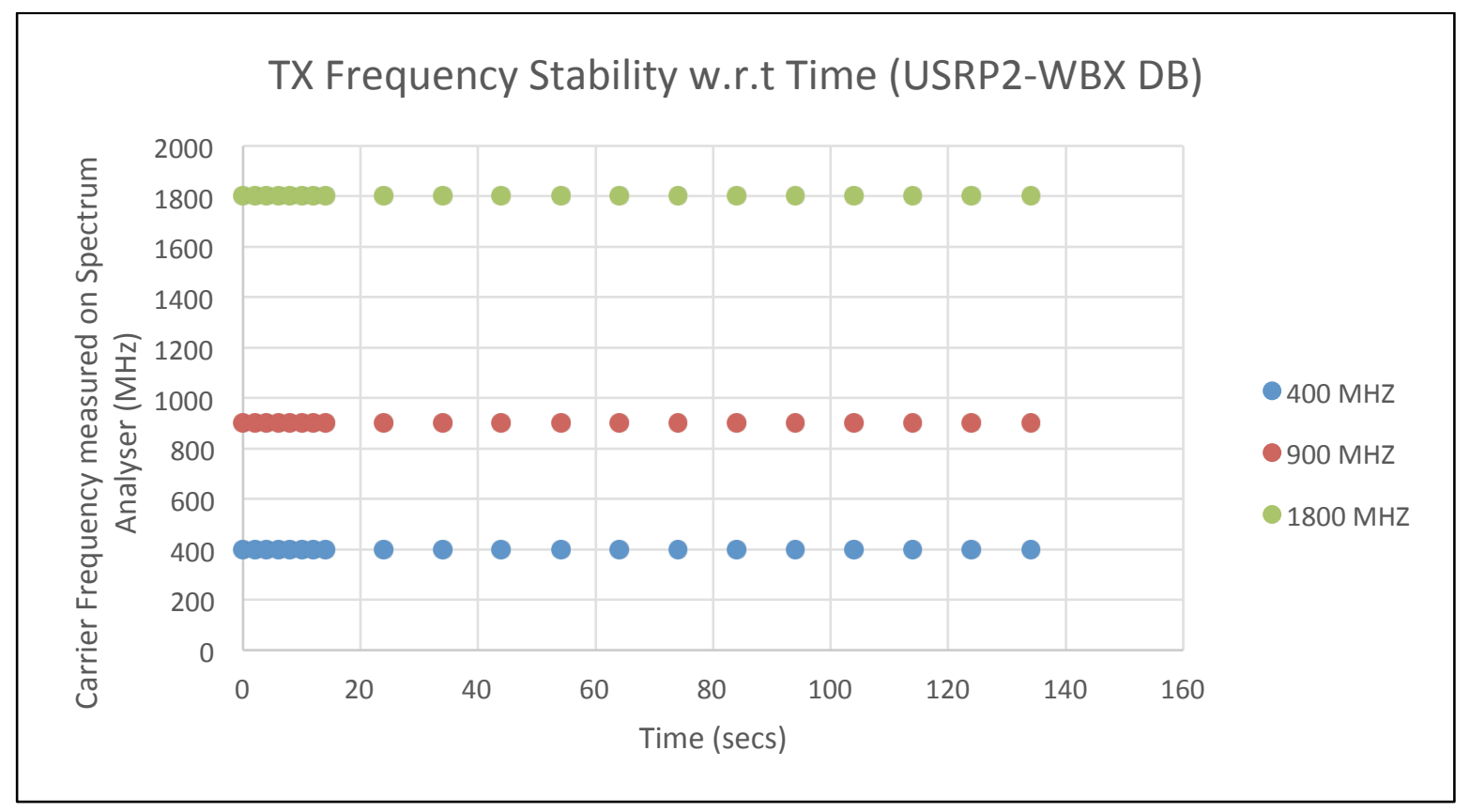

Figure 4.15 Transmitted Frequency Stability Test (USRP2-WBX DB)

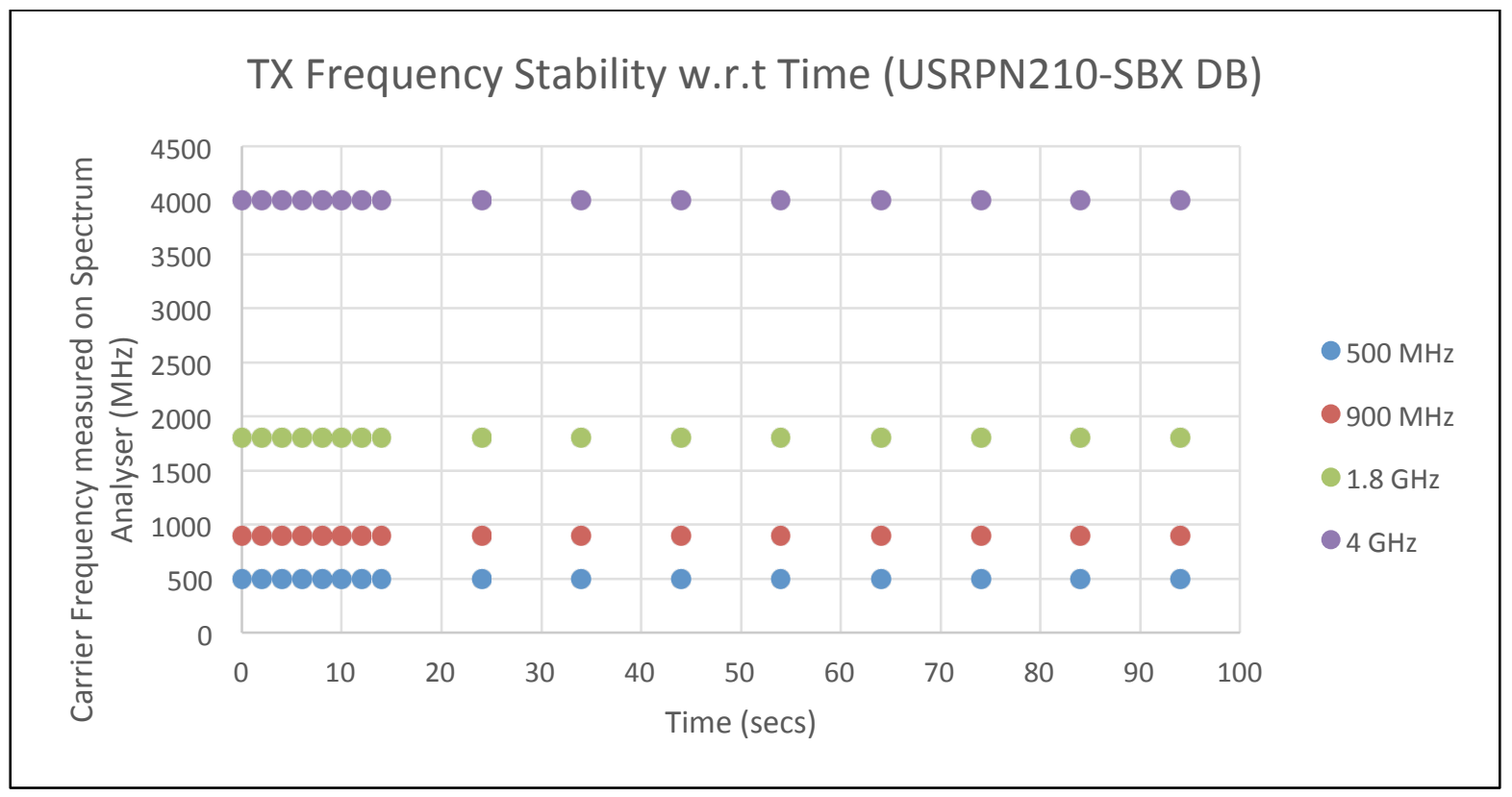

Figure 4.16 Transmitted Frequency Stability Test (USRPN210-SBX DB) 


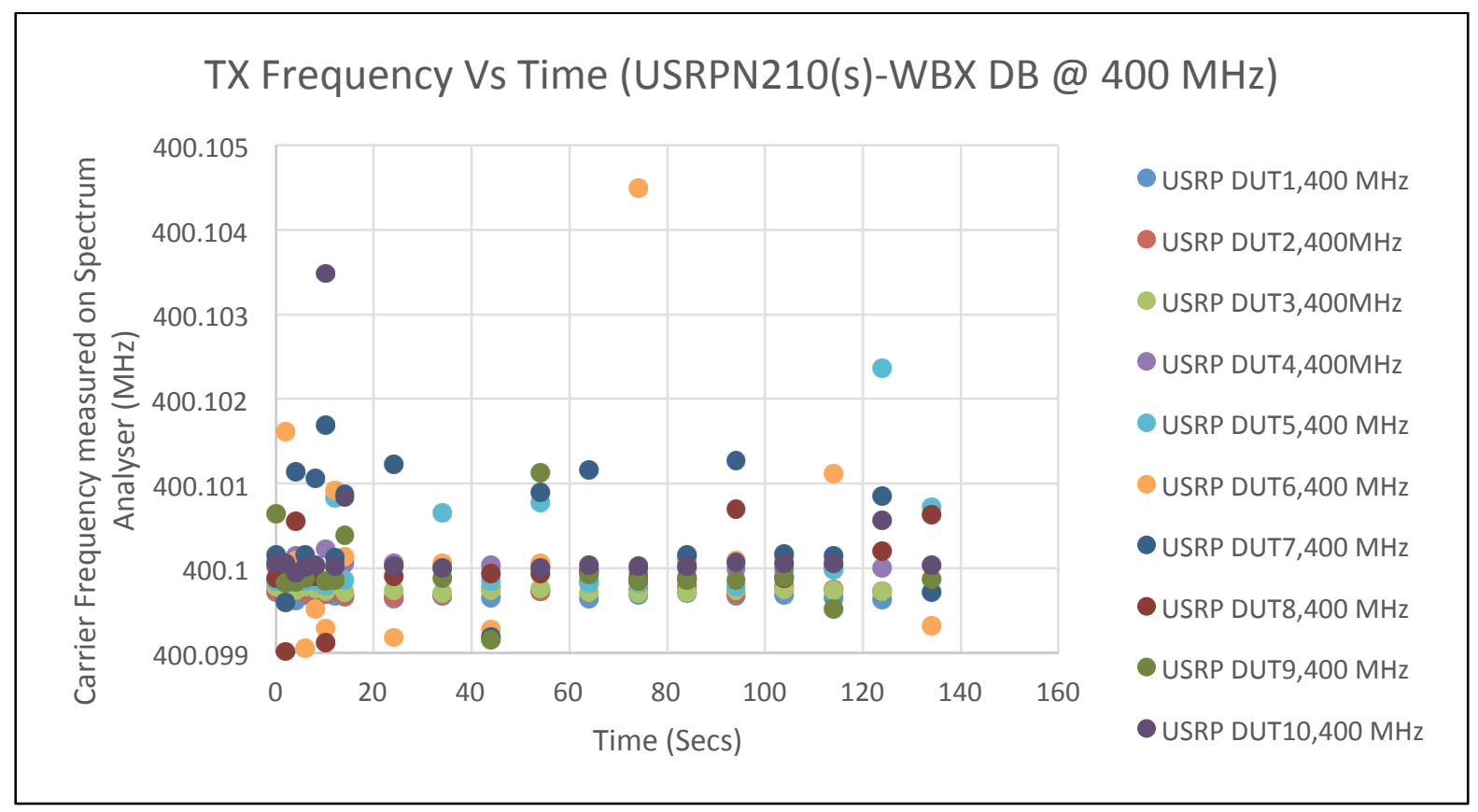

Figure 4.17 Transmitted Frequency Variation with Time @ $400 \mathrm{MHz}$

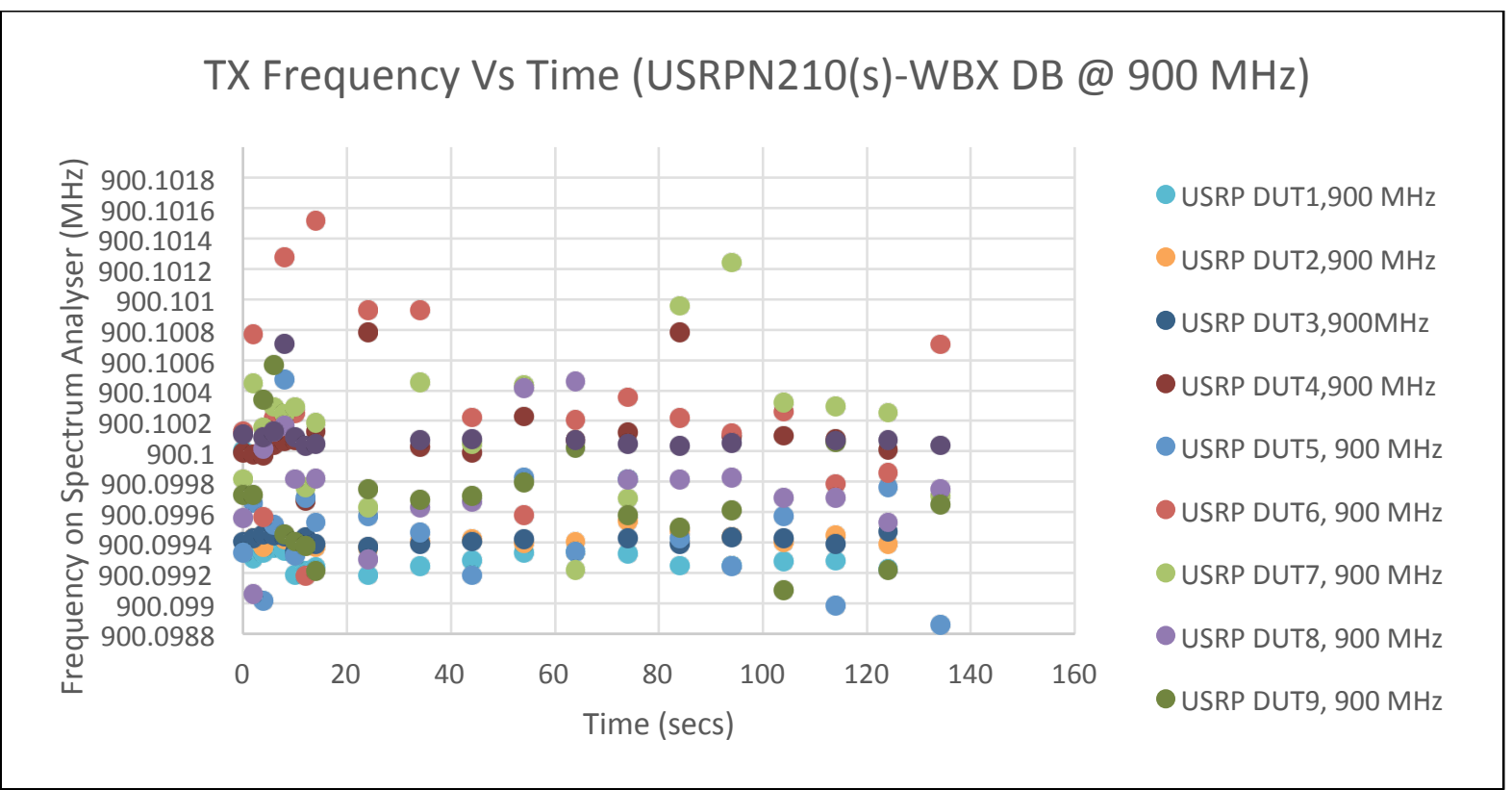

Figure 4.18 Transmitted Frequency Variation with Time@9 900 MHz 


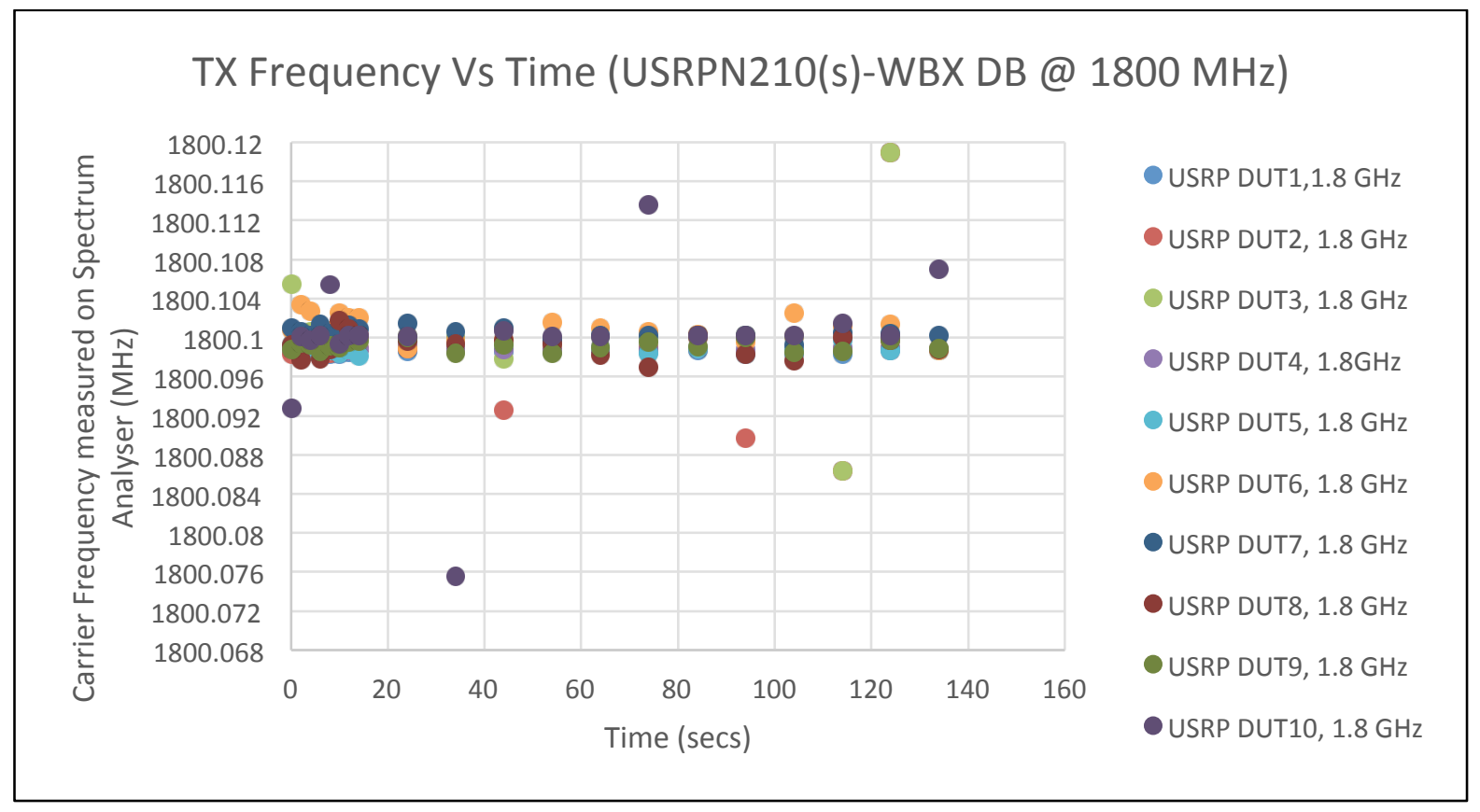

Figure 4.19 Transmitted Frequency Variation with Time@1800 MHz

\subsubsection{Transmitted Amplitude Stability Test Procedure}

1. Connect the USRPN210 to the HOST PC using gigabit Ethernet cable (blue in the picture)

2. Connect the USRP N210 TX port (RF1) to the input of Spectrum Analyzer via an adjustable attenuator to provide $30 \mathrm{~dB}$ attenuation before the input is given to spectrum analyzer to prevent any damages.

3. The spectrum analyzer is set on Real time Sample and Hold mode. The RBW set to 1 $\mathrm{MHz}$, the acquiring time gets set to $51.2 \mathrm{~s}$ while the processing time is $51.2 \mathrm{sec}$. frequency.

4. Choose the carrier frequency measurement that can be explicitly to get the real time measurement of the RF carrier frequency on the spectrum Analyzer. 
5. The UHD_SIGGEN.py is executed with the following input parameters: - Amplitude $=1$,$\mathrm{s}=1 \mathrm{e} 6,-\mathrm{f} 400 \mathrm{e} 6,-\mathrm{x} 100 \mathrm{e} 3-$ gain 0

6. A sine wave signal is produced at $400.1 \mathrm{MHz}$ and hence the center frequency on the spectrum analyzer is also set as $400.1 \mathrm{MHz}$.

7. After approximately 2 mins, the signal would be visible on the spectrum analyzers screen. Press the "PEAK" button to obtain the peak power value of the signal and also the actual value of the carrier frequency at which the peak occurs. Note this value down.

8. After approximately 2 mins again the value would be updated. Note down the readings at the interval of every 2 mins for the first 1-hour, then at an interval of 10 mins for next hour.

9. Repeat the steps from 5 to 8 for $900 \mathrm{MHz}$ and $1.800 \mathrm{MHz}$ in line with other tests. The UHD_SIGGEN parameters will change accordingly $(-\mathrm{f}=900 \mathrm{e} 6$ and $-\mathrm{f}=1800 \mathrm{e} 6$ resp.)

10. The readings have been tabulated in table 4.8 for the 10 devices at the 3 different frequencies. It can be seen that the deviation show a similar trend and transmitted power is stable over the period of experimentation.

11. This experiment was also repeated for a USRP2 with WBX board and USRPN210 with SBX board.

12. The readings show that the USRPN210 with SBX has a lower power since $60 \mathrm{~dB}$ of attenuation was applied before input to spectrum analyzer. The USPR2 with WBX board shows anomalous behavior with lower power of $\sim 38.4 \mathrm{dBm}$ at $1800 \mathrm{MHz}$ while a higher power of $\sim 41.45 \mathrm{dBm}$ at $400 \mathrm{MHz}$. 
Table 4.8 Readings for Transmitted Power Stability Test

\begin{tabular}{|c|c|c|c|}
\hline \multirow{3}{*}{$\begin{array}{c}\text { USRP \# } \\
\begin{array}{c}\text { USRPN210(s) with WBX daughter } \\
\text { boards }\end{array} \\
\end{array}$} & \multirow{2}{*}{\multicolumn{3}{|c|}{$\begin{array}{c}\text { Average Transmitted Power (over a period of } 1 \\
\text { hour) }(\mathrm{dBm})\end{array}$}} \\
\hline & & & \\
\hline & $400 \mathrm{MHz}$ & $900 \mathrm{MHz}$ & $1800 \mathrm{MHz}$ \\
\hline \multicolumn{4}{|l|}{$\begin{array}{l}\text { USRPN210(s) with WBX daughter } \\
\text { boards }\end{array}$} \\
\hline VT361926 & -36.3 & -39.72 & -49.41 \\
\hline VT361927 & -35.69 & -39.42 & -48.33 \\
\hline VT361928 & -35.75 & -39.67 & -47.44 \\
\hline VT361929 & -36.35 & -39.24 & -47.77 \\
\hline VT361930 & -35.61 & -39.34 & -47.59 \\
\hline VT361931 & -35.47 & -38.89 & -46.48 \\
\hline VT361932 & -35.93 & -39.33 & -47.34 \\
\hline VT361933 & -35.48 & -38.65 & -46.45 \\
\hline VT361936 & -35.59 & -39.03 & -46.65 \\
\hline VT361937 & -35.19 & -38.82 & -46.41 \\
\hline $\begin{array}{c}\text { Mean Transmitted Power (Averaged } \\
\text { over all the USRP(s) ) }\end{array}$ & -35.74 & -39.21 & -47.39 \\
\hline Standard Deviation & 0.37 & 0.36 & 0.96 \\
\hline $95 \%$ confidence Interval & 0.23 & 0.22 & 0.6 \\
\hline Lower bound & -35.97 & -39.43 & -47.99 \\
\hline Upper bound & -35.51 & -38.99 & -46.79 \\
\hline Maximum value & -35.19 & -38.65 & -46.41 \\
\hline Minimum value & -36.35 & -39.72 & -49.41 \\
\hline Range of values & 1.16 & 1.07 & 3.00 \\
\hline USRP2-1563 & -41.45 & -37.92 & -38.41 \\
\hline USRPN210-SBX & -56.29 & -57.18 & -62.79 \\
\hline
\end{tabular}




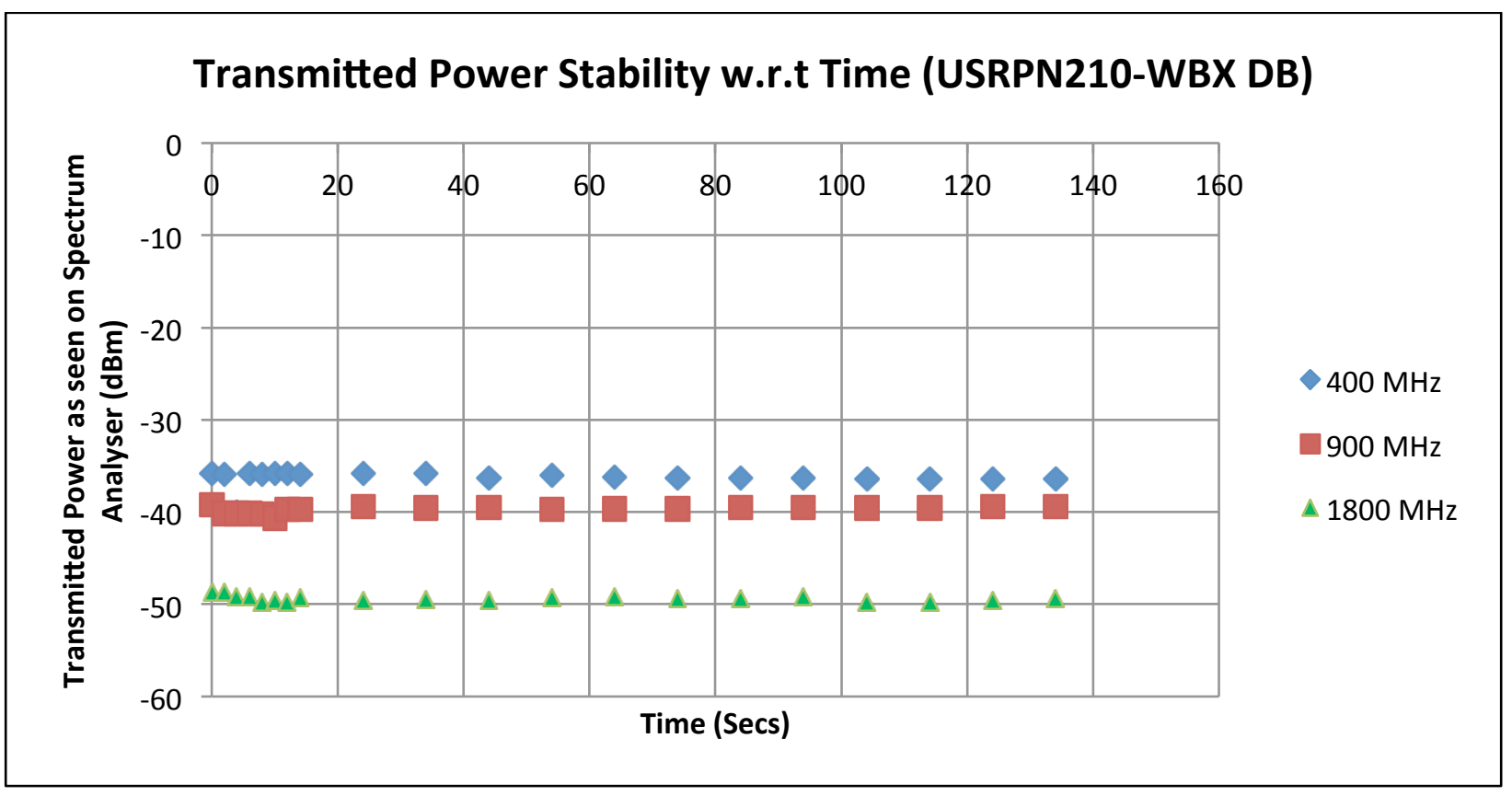

Figure 4.20 Transmitted Power Stability Test (USRPN210-WBX DB)

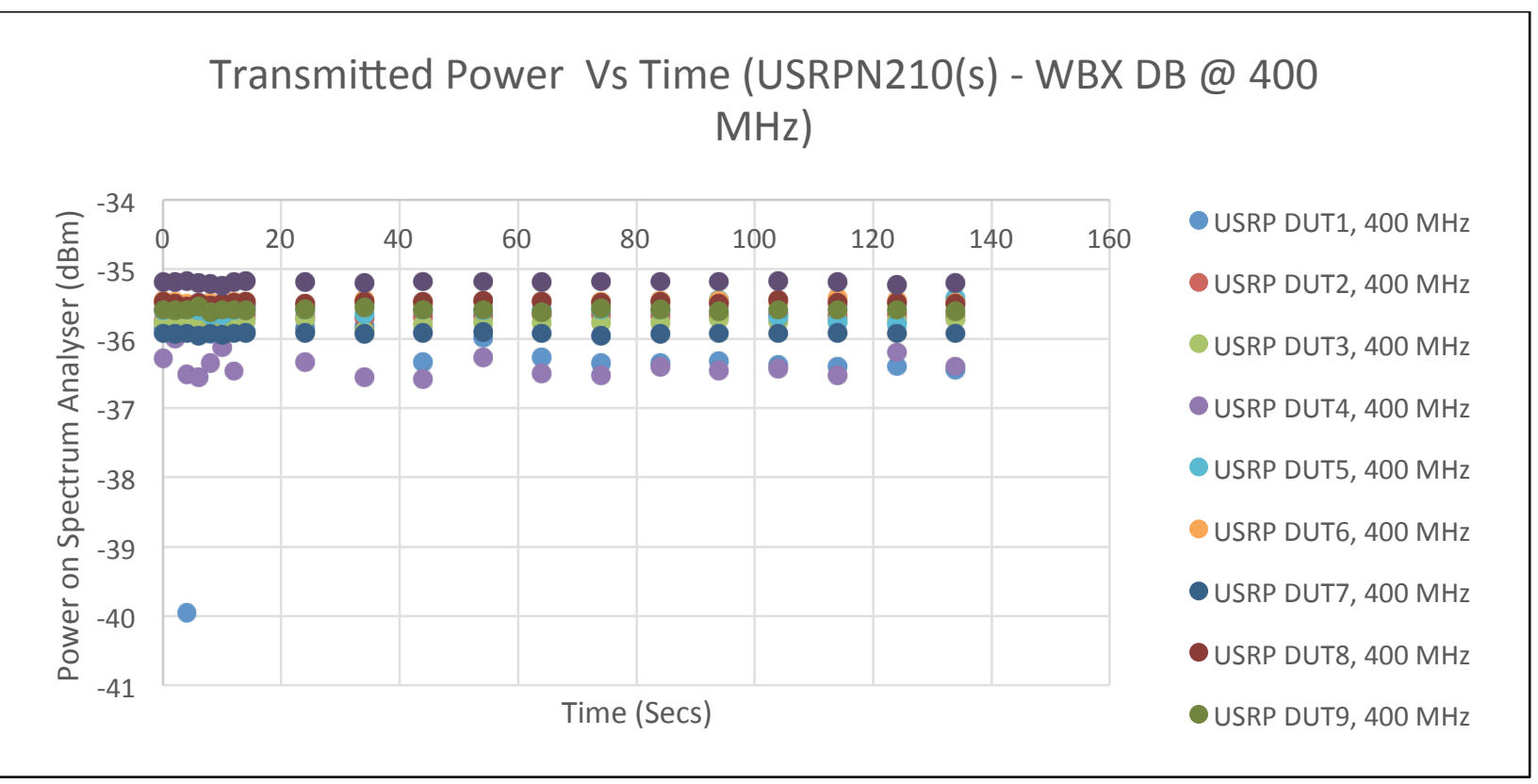

Figure 4.21 Transmitted Power Variation with Time @ $400 \mathrm{MHz}$ 


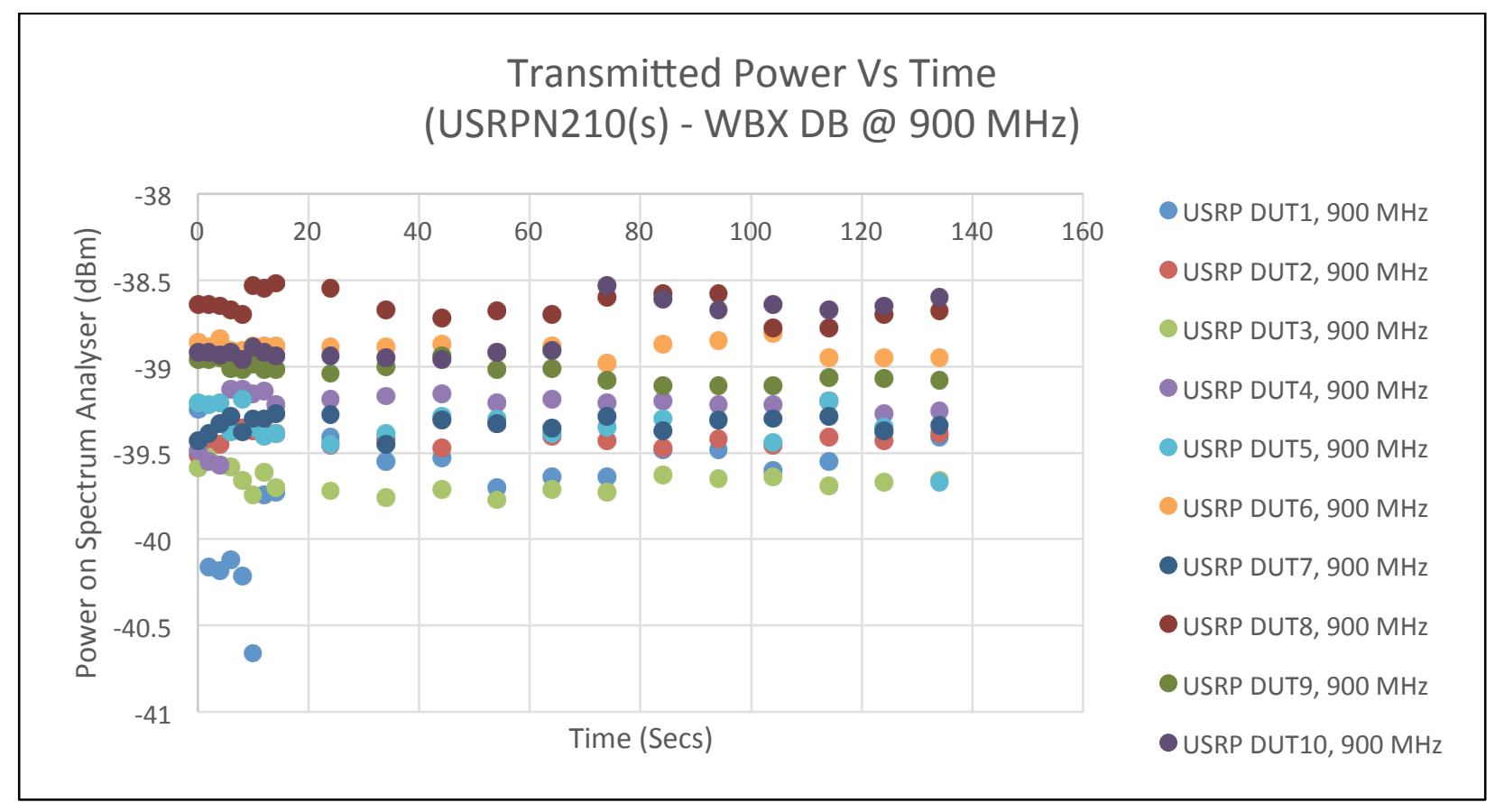

Figure 4.22 Transmitted Power Variation with Time @ 900 MHz

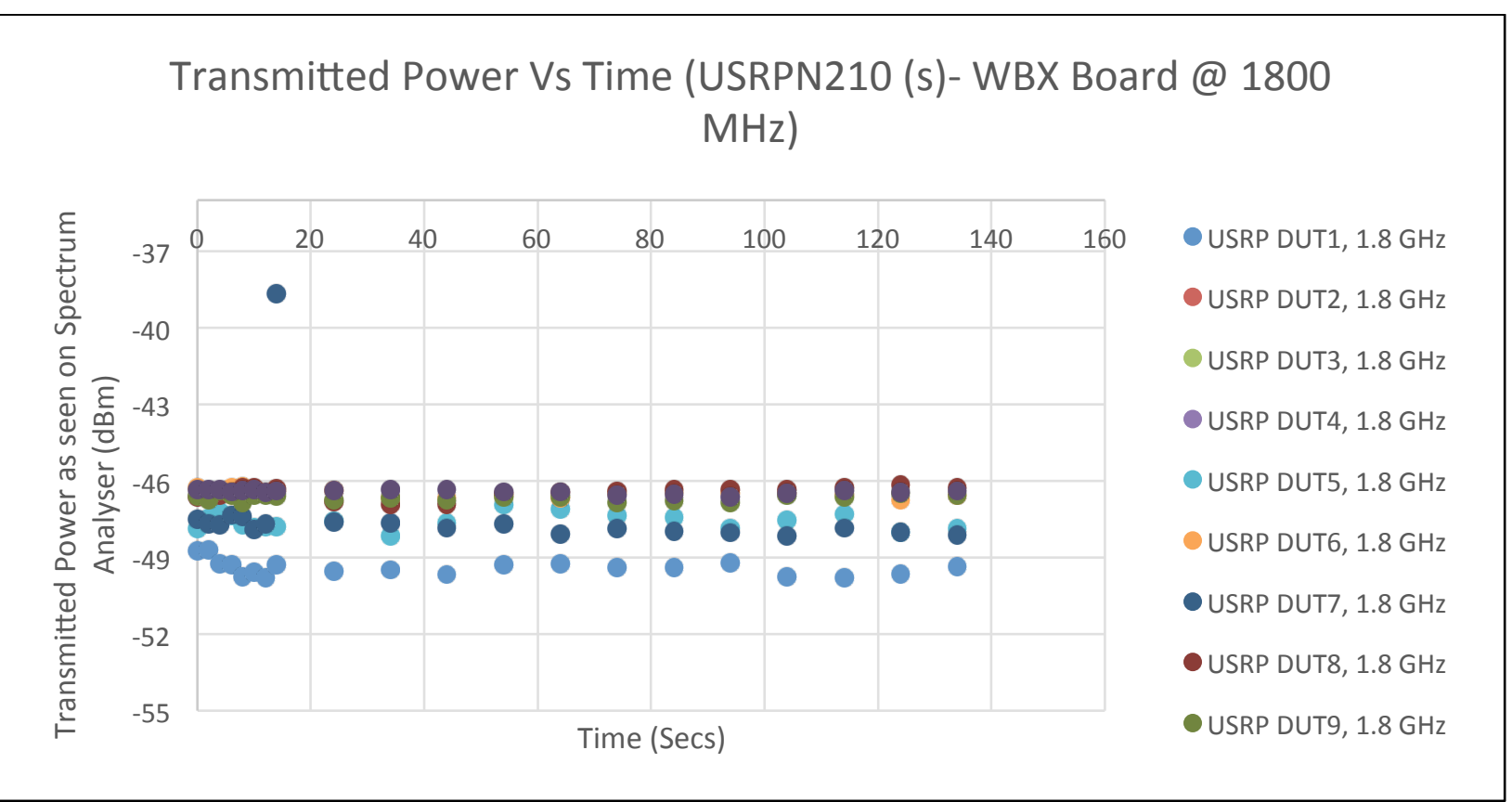

Figure 4.23 Transmitted Power Variation with Time @ 1800 MHz 


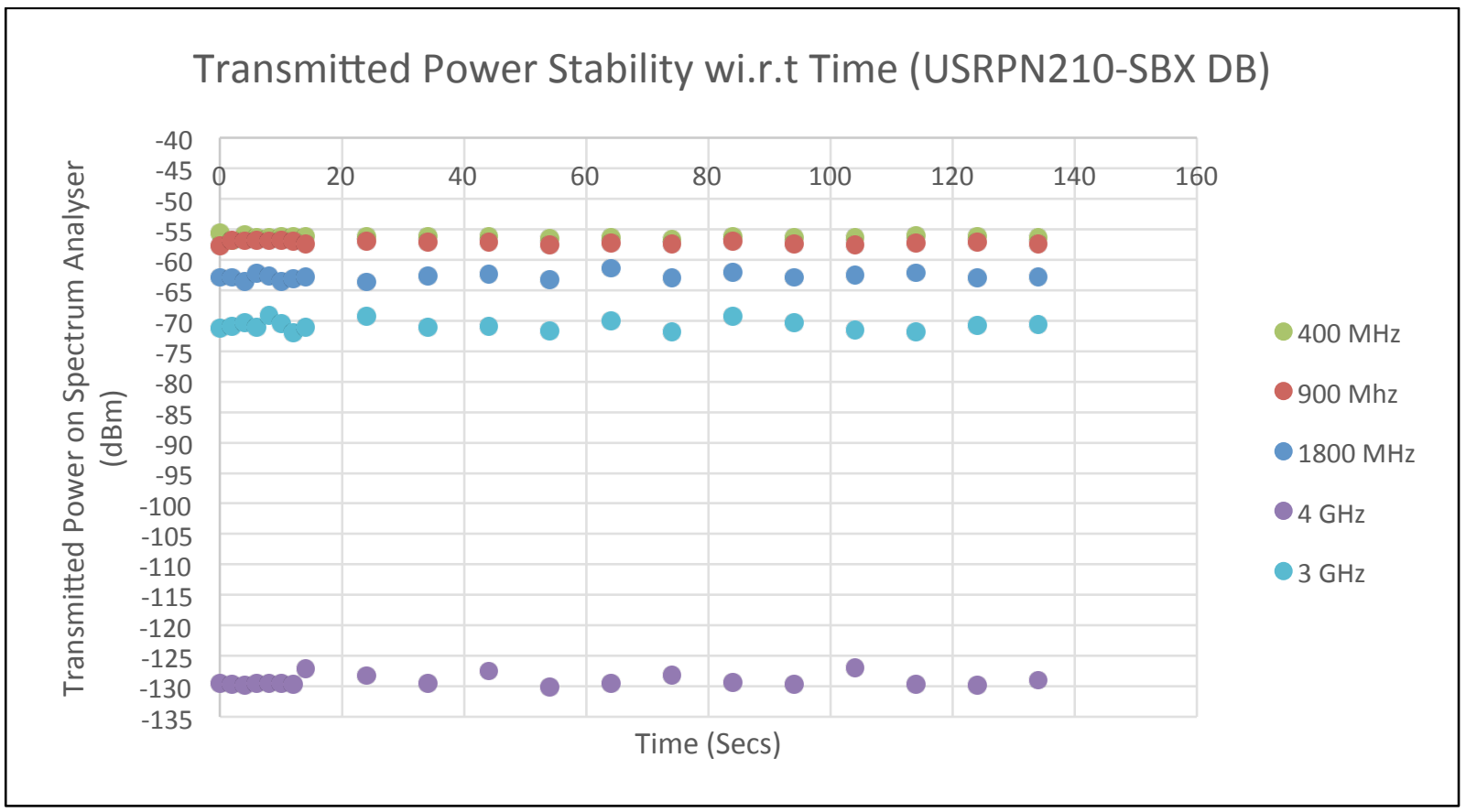

Figure 4.24 Transmitted Power Stability Test (USRPN210-SBX DB)

\section{Transmitted Power Stability w.r.t Time (USRPN210 - WBX DB, GRC custom Flowgraph)}

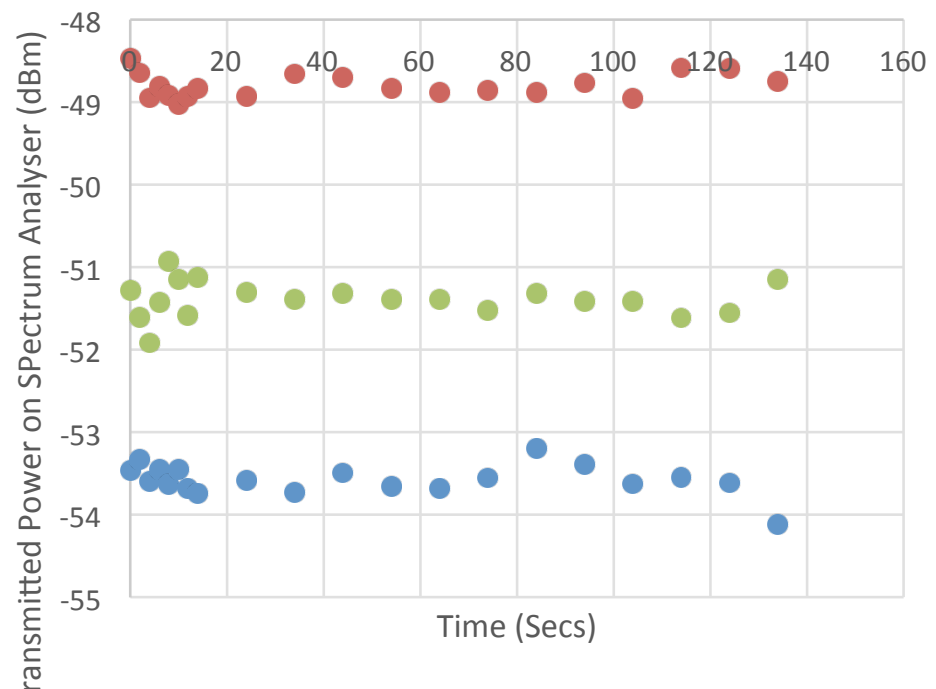

USRP\#30,GRC - $0 \mathrm{~dB}$ atten, $400 \mathrm{MHz}$

USRP\#30, GRC - $0 \mathrm{~dB}$ atten, $900 \mathrm{MHz}$

USRP\#30, GRC - $0 \mathrm{~dB}$ atten, 1800 $\mathrm{MHz}$

Figure 4.25 Transmitted Power Stability Test (Custom Flow Graph, USRPN210-WBX DB) 


\subsubsection{Transmitted Signal Power Variation with TX Gain}

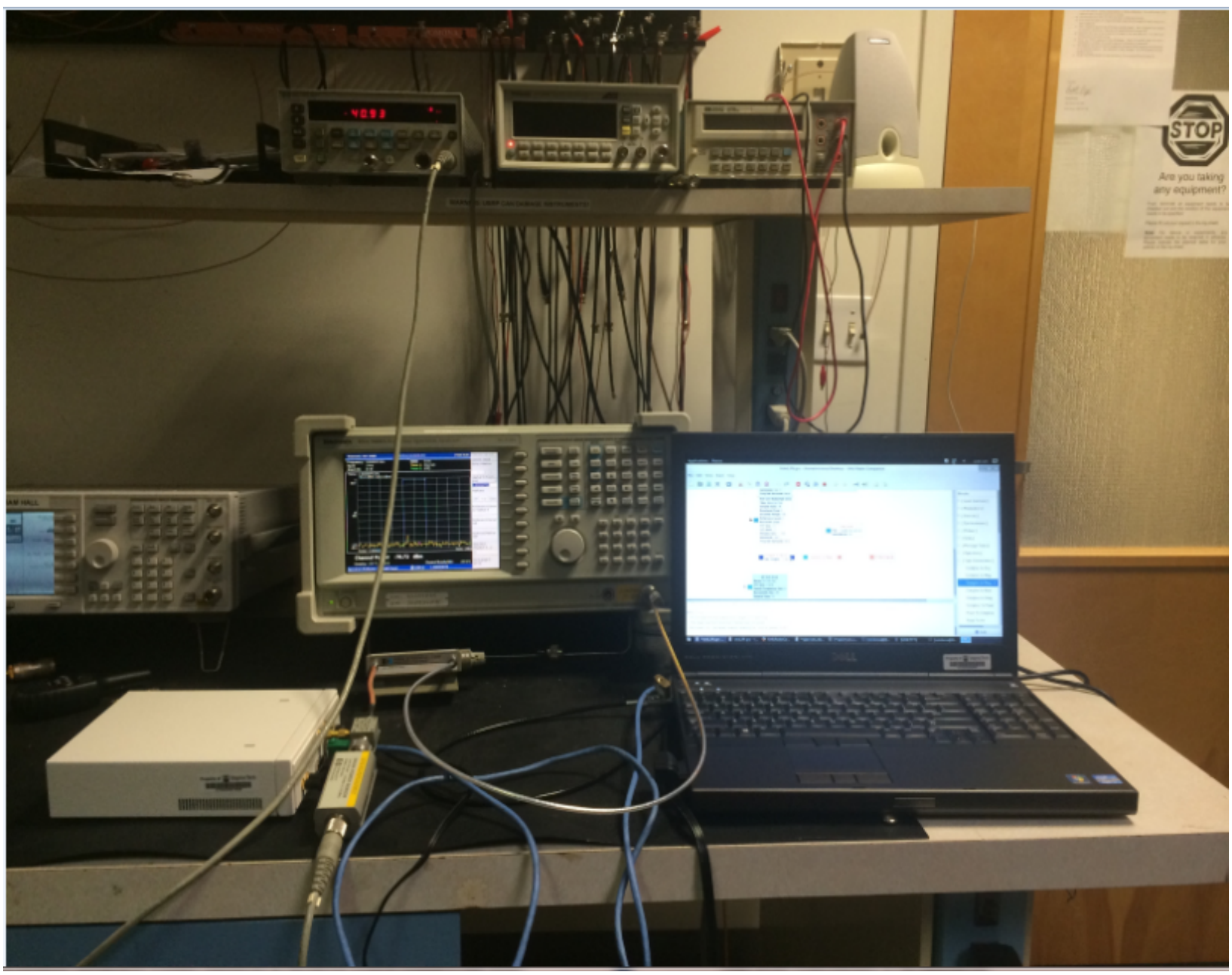

Figure 4.26 Laboratory Setup for TX Power Variation with TX Gain Test

\subsubsection{Single Tone Test}

1. Connect the USRPN210 to the HOST PC using gigabit Ethernet cable (blue in the picture)

2. Connect the USRP N210 TX port (RF1) to the input of a power splitter with 2 output ports. Connect one output to the Spectrum Analyzer via an adjustable attenuator to 
provide required attenuation to prevent any damages. The spectrum analyzer is set on Real time Spectrum Analyzer mode.

3. Connect the second output port of the power combiner to a power meter to get average power over the entire operational bandwidth. This is just to compare that the power displayed on both spectrum analyzer and power meter

4. The UHD_SIGGEN.py is executed with the following input parameters:

-Amplitude $=0.707$, -s 1e6, -f 400e6, -x 100e3, -gain 0

5. A sine wave signal is produced at $400.1 \mathrm{MHz}$ and hence the center frequency on the spectrum analyzer is also set as $400.1 \mathrm{MHz}$.

6. When the output signal is visible on the spectrum analyzers screen press the "PEAK" button to obtain the peak power value of the signal and also the actual value of the carrier frequency at which the peak occurs. Note these values down.

7. Note the corresponding reading from the power meter too in $\mathrm{dBm}$ and watts if required.

8. Stop the UHD_SIGGEN.py. Now set the increment the gain parameter by $2 \mathrm{~dB}$ i.e. the UHD_SIGGEN.py parameters are set as - Amplitude $=0.707$, - s 1e6, -f 400e6,-x 100e3, -gain 2

9. Again note down the readings from spectrum analyzer and power meter.

10. Repeat steps 6 to 10 with gain incremented every time in steps $2 \mathrm{~dB}$ until $30 \mathrm{~dB}$ to cover the entire range of WBX daughter board.

11. Repeat the steps from 5 to 8 for $900 \mathrm{MHz}$ and $1.800 \mathrm{MHz}$ in line with other tests. The UHD_SIGGEN.py parameters will change accordingly $(-\mathrm{f}=900 \mathrm{e} 6$ and $-\mathrm{f}=1800 \mathrm{e} 6 \mathrm{resp}$. 
12. The readings have been tabulated in Table 4.9 for the USRPN210 device with WBX daughterboard at the 3 different frequencies. Increase in gain is seen to produce proportional raise in output power readings. The power combiner produces a loss $-3 \mathrm{~dB}$ and hence needs to be accounted for in the readings obtained on spectrum analyzer and power meter.

13. This experiment was also repeated for a USRPN210 with SBX board.

\subsubsection{Two Tone Test}

1. Connect the USRPN210 to the HOST PC using gigabit Ethernet cable (blue in the picture)

2. Connect the USRP N210 TX port (RF1) to the input of a power splitter with 2 output ports. Connect one output to the Spectrum Analyzer via an adjustable attenuator to provide required attenuation to prevent any damages. The spectrum analyzer is set on Real time Spectrum Analyzer mode.

3. Connect the second output port of the power combiner to a power meter to get average power over the entire operational bandwidth. This is just to compare that the power displayed on both spectrum analyzer and power meter

4. Choose the carrier frequency measurement that can be explicitly to get the real time measurement of the RF carrier frequency on the spectrum Analyzer.

5. The UHD_SIGGEN.py is executed with the following input parameters:

-Amplitude $=0.354$, -s 1e6, -f 400e6, -x 50e3, -y 75e3, -gain 0 
6. Two sine wave signals are produced at $400.050 \mathrm{MHz}$ and $400.075 \mathrm{MHz}$, which are the two fundamental tones. The center frequency on the spectrum analyzer is also set as 400.0 MHz.

7. When the output signals are visible on the spectrum analyzers screen press the "PEAK" button to obtain the peak power value of the signals (marker frequency can be set for getting the peak values) and also the actual value of the carrier frequency at which the peak occurs. Note these values down.

8. Note the corresponding reading from the power meter too in $\mathrm{dBm}$ and watts if required. This would actually be a normalized power of the two tones and hence around $3 \mathrm{~dB}$ above the Spectrum analyzer reading.

9. Apart from these two fundamental tones at $400.050 \mathrm{MHz}$ and $400.075 \mathrm{MHz}$, intermodulation products would occur at $400.025 \mathrm{MHz}$ and $400.1 \mathrm{MHz}$. Note the readings at these two frequencies too.

10. Stop the UHD_SIGGEN.py. Now set the increment the gain parameter by $2 \mathrm{~dB}$ i.e. the UHD_SIGGEN.py parameters are set as:

-Amplitude $=0.354,-$ s 1e6, -f 400e6, -x 050e3, -y 075e3, -gain 2

11. Again note down the readings from spectrum analyzer and power meter.

12. Repeat steps 6 to 10 with gain incremented every time in steps $2 \mathrm{~dB}$ until $30 \mathrm{~dB}$ to cover the entire range of WBX daughter board.

13. Repeat the steps from 5 to 8 for $900 \mathrm{MHz}$ and $1.800 \mathrm{MHz}$ in line with other tests. The UHD_SIGGEN.py parameters will change accordingly $(-\mathrm{f}=900 \mathrm{e} 6$ and $-\mathrm{f}=1800 \mathrm{e} 6$ resp.). The IMD products would also occur at respective offset of fundamental tones. 
14. The readings have been tabulated in Table 4.10 for the USRPN210 device with WBX daughterboard at the 3 different frequencies. Increase in gain is seen to produce proportional raise in the IMD products and their influence would result in compression of fundamental tones.

15. The power combiner produces a loss $3 \mathrm{~dB}$ and hence $3 \mathrm{~dB}$ must be added to the reading obtained on the spectrum analyzer and power meter.

16. This experiment was also repeated for a USRPN210 with SBX board and USRP2 with WBX board.

17. It can be seen for USRPN210 with WBX board, the variation of single tone power with input TX gain is linear until $31 \mathrm{~dB}$, but for USRPN210 with SBX DB, the output power seems to saturate near $25 \mathrm{~dB}$ for all 3 frequencies. Hence the linear range of the USRPB210 with SBX board could be said to be 0 to $20 \mathrm{~dB}$ of TX gain with $1 \mathrm{dBC}$ point occurring $\sim 22 \mathrm{~dB}$ at $500 \mathrm{MHz}, \sim 24 \mathrm{~dB}$ at $900 \mathrm{MHz}$ and $\sim 28 \mathrm{~dB}$ at $1800 \mathrm{MHz}$. Similarly for USRP2 device, the output power completely saturates from $25 \mathrm{dBm}$ and the safe linear range could be said to range from 5 to $23 \mathrm{~dB}$ at 400, 900 and $1800 \mathrm{MHz}$.

18. As for the inter-modulation distortion, the USRPN210 with WBX device seems to be unaffected by them until $31 \mathrm{dBm}$ as their power do not raise above $-30 \mathrm{dBm}$ at all 3 frequencies. But in case of USRPN210 with SBX board, the high power inter-modulation distortions (Pimd $>=-30 \mathrm{dBm}$ ) occur from $-27 \mathrm{~dB}$ at $500 \mathrm{MHz}$ and $29 \mathrm{~dB}$ at $900 \mathrm{MHz}$, At $1800 \mathrm{MHz}$ the distortions raise above $-40 \mathrm{dBm}$ at $30 \mathrm{dBm}$ and hence affect the output slightly only from this gain value. For USRP2 with WBX board, the inter-modulation power immediately peaks above $-30 \mathrm{dBm}$ from 2 to $12 \mathrm{~dB}$ of TX gain at $400 \mathrm{MHz}$ and 
then drops considerably for rest of the gain range. At 900 and $1800 \mathrm{MHz}$, the IMD power peaks above $-40 \mathrm{dBm}$ from 5 to $10 \mathrm{~dB}$ gain at $900 \mathrm{MHz}$ and 8 to $10 \mathrm{~dB}$ gain at 1800 $\mathrm{MHz}$, they do not affect the output much.

Table 4.9 Readings for Transmitted Power Variation with Gain Test, Single Tone

\begin{tabular}{|c|c|c|c|c|c|c|}
\hline Ampl & 0.707 & Device & $\begin{array}{c}\text { USRPN210 + } \\
\text { WBX DB }\end{array}$ & Test & \multirow{2}{*}{\multicolumn{2}{|c|}{$\begin{array}{c}\text { Single Tone Transmitter Power } \\
\text { Vs TX Gain } \\
1800 \mathrm{MHz}\end{array}$}} \\
\hline Frequency & \multicolumn{2}{|c|}{$400 \mathrm{MHz}$} & \multicolumn{2}{|c|}{$900 \mathrm{MHz}$} & & \\
\hline TX GAIN & $\begin{array}{l}\text { Spectrum } \\
\text { Analyzer } \\
\text { Readings } \\
\text { (dBm) }\end{array}$ & $\begin{array}{l}\text { Power } \\
\text { Meter } \\
\text { Readings } \\
\text { (dBm) }\end{array}$ & $\begin{array}{c}\text { Spectrum } \\
\text { Analyzer } \\
\text { Readings }(\mathrm{dBm})\end{array}$ & $\begin{array}{l}\text { Power } \\
\text { Meter } \\
\text { Readings } \\
\text { (dBm) }\end{array}$ & $\begin{array}{c}\text { Spectrum } \\
\text { Analyzer } \\
\text { Readings (dBm) }\end{array}$ & $\begin{array}{l}\text { Power Meter } \\
\text { Readings } \\
\text { (dBm) }\end{array}$ \\
\hline 0 & -8.94 & -12.52 & -16.15 & -16.3 & -22.54 & -22.1 \\
\hline 2 & -10.03 & -10.6 & -14.25 & -14.36 & -20.59 & -20.17 \\
\hline 4 & -8.03 & -8.59 & -12.21 & -12.33 & -18.59 & -18.18 \\
\hline 5 & -6.89 & -7.6 & -10.21 & -10.33 & -16.53 & -16.15 \\
\hline 6 & -6.07 & -6.64 & -11.25 & -11.35 & -17.55 & -17.16 \\
\hline 8 & -4.36 & -4.92 & -8.51 & -8.62 & -14.64 & 14.23 \\
\hline 10 & -2.4 & -2.95 & -6.58 & -6.66 & -12.66 & -12.24 \\
\hline 12 & -0.34 & -0.9 & -4.5 & -4.58 & -10.61 & -10.17 \\
\hline 14 & 1.65 & 1.09 & -2.52 & -2.57 & -8.56 & -8.12 \\
\hline 15 & 2.64 & 2.08 & -1.52 & -1.58 & -7.54 & -7.07 \\
\hline 16 & 3.5 & 2.95 & -0.57 & -0.62 & -6.53 & -6.06 \\
\hline 18 & 5.46 & 4.91 & 1.37 & 1.33 & -4.57 & -4.09 \\
\hline 20 & 7.51 & 6.96 & 3.41 & 3.37 & -2.51 & -2.03 \\
\hline 22 & 9.49 & 8.92 & 5.4 & 5.35 & -0.49 & 0 \\
\hline 24 & 11.4 & 10.83 & 7.27 & 7.23 & 1.53 & 2.02 \\
\hline 25 & 12.52 & 11.78 & 8.21 & 8.16 & 2.52 & 3.01 \\
\hline 26 & 13.5 & 12.78 & 9.21 & 9.14 & 3.51 & 3.99 \\
\hline 28 & 15.48 & 14.78 & 11.22 & 11.15 & 5.57 & 6.06 \\
\hline 30 & 17.14 & 16.43 & 13.12 & 13.01 & 7.55 & 8.03 \\
\hline 31 & 17.65 & 16.98 & 13.95 & 13.84 & 8.52 & 9 \\
\hline
\end{tabular}


19. A power reference table was constructed from the single tone test readings, which could prove useful when trying to use transmitter power for any wireless applications.

For e.g. the o/p power at ampl $=1$ at TX gain of 0 is $4.09 \mathrm{~dB}$ which is $3 \mathrm{dBm}$ higher than $\mathrm{o} / \mathrm{p}$ power when $\mathrm{ampl}=0.707(\mathrm{TX}$ gain $=0)$ and $6 \mathrm{dBm}$ higher than $\mathrm{o} / \mathrm{p}$ power measured at $\mathrm{ampl}=0.5(\mathrm{TX}$ gain $=0)$. This is as per expectations.

$[20 \log (0.5)=-6 \mathrm{~dB}$ and $20 \log (0.707)=-3 \mathrm{~dB}]$

With this the transmitter power output from TX (RF1) port for the device can closely estimated.

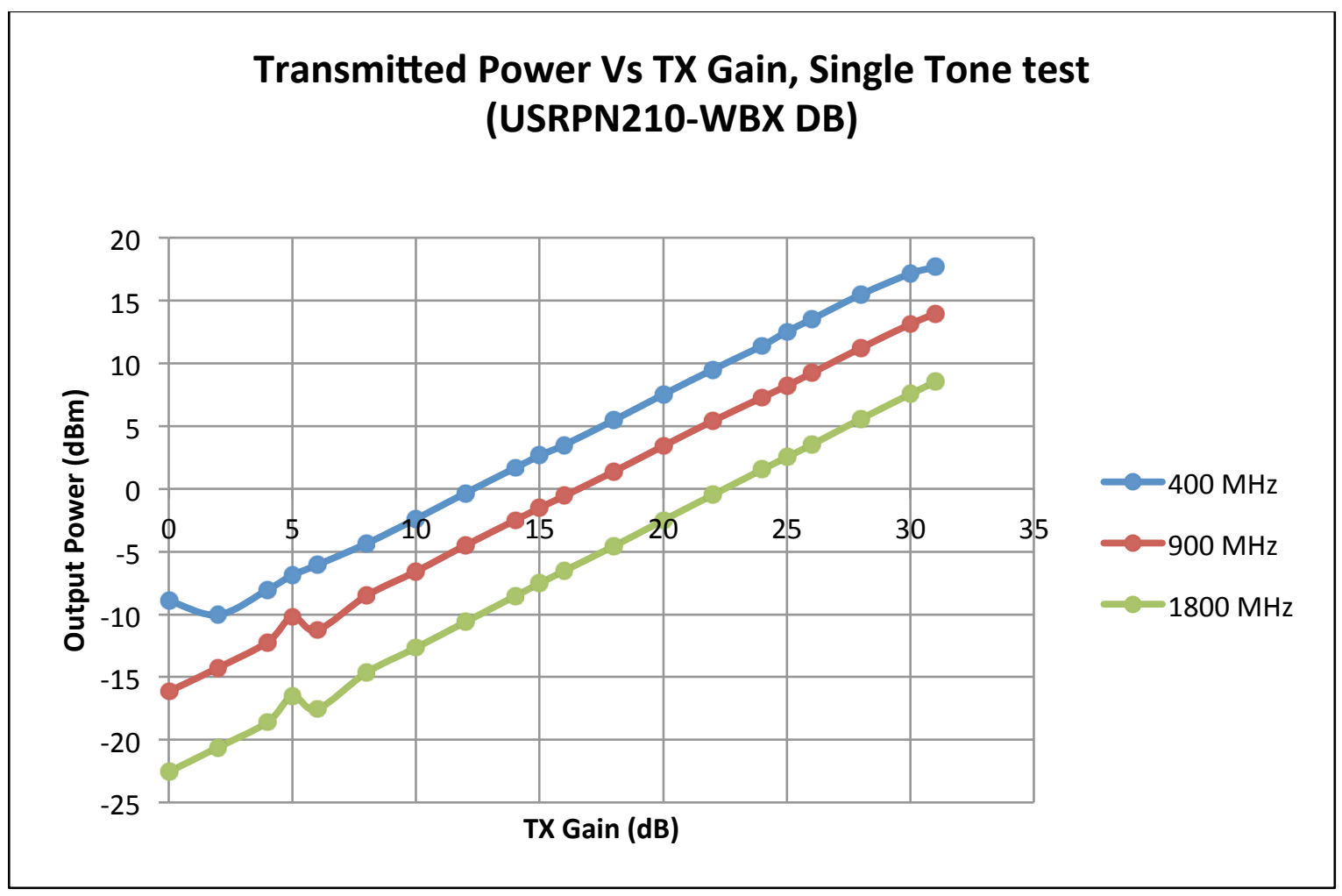

Figure 4.27 Transmitted Power Variation with TX Gain (USRPN210-WBX DB) 
Table 4.10 Readings for Transmitted Power Variation with Gain Test, Two Tone

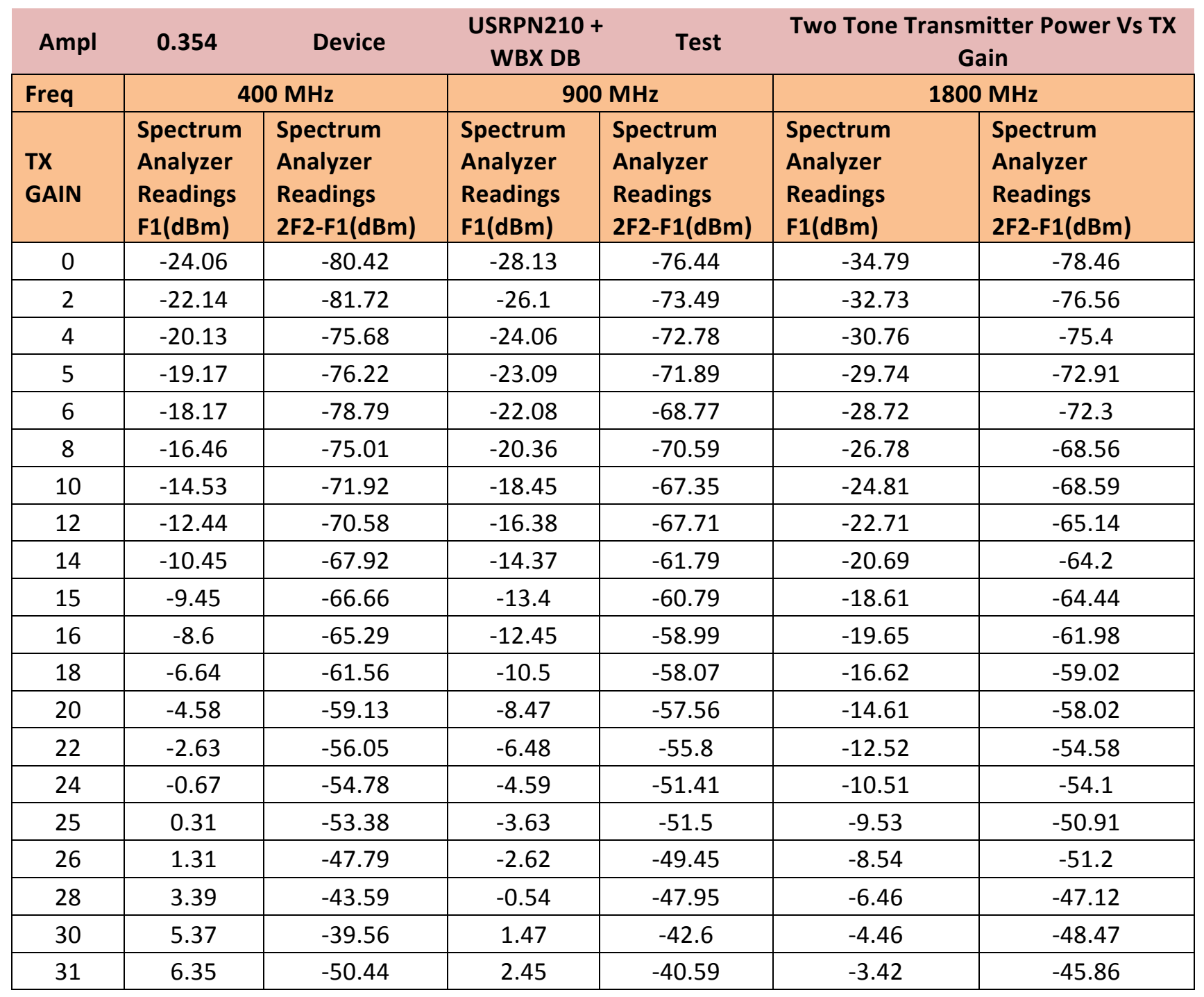




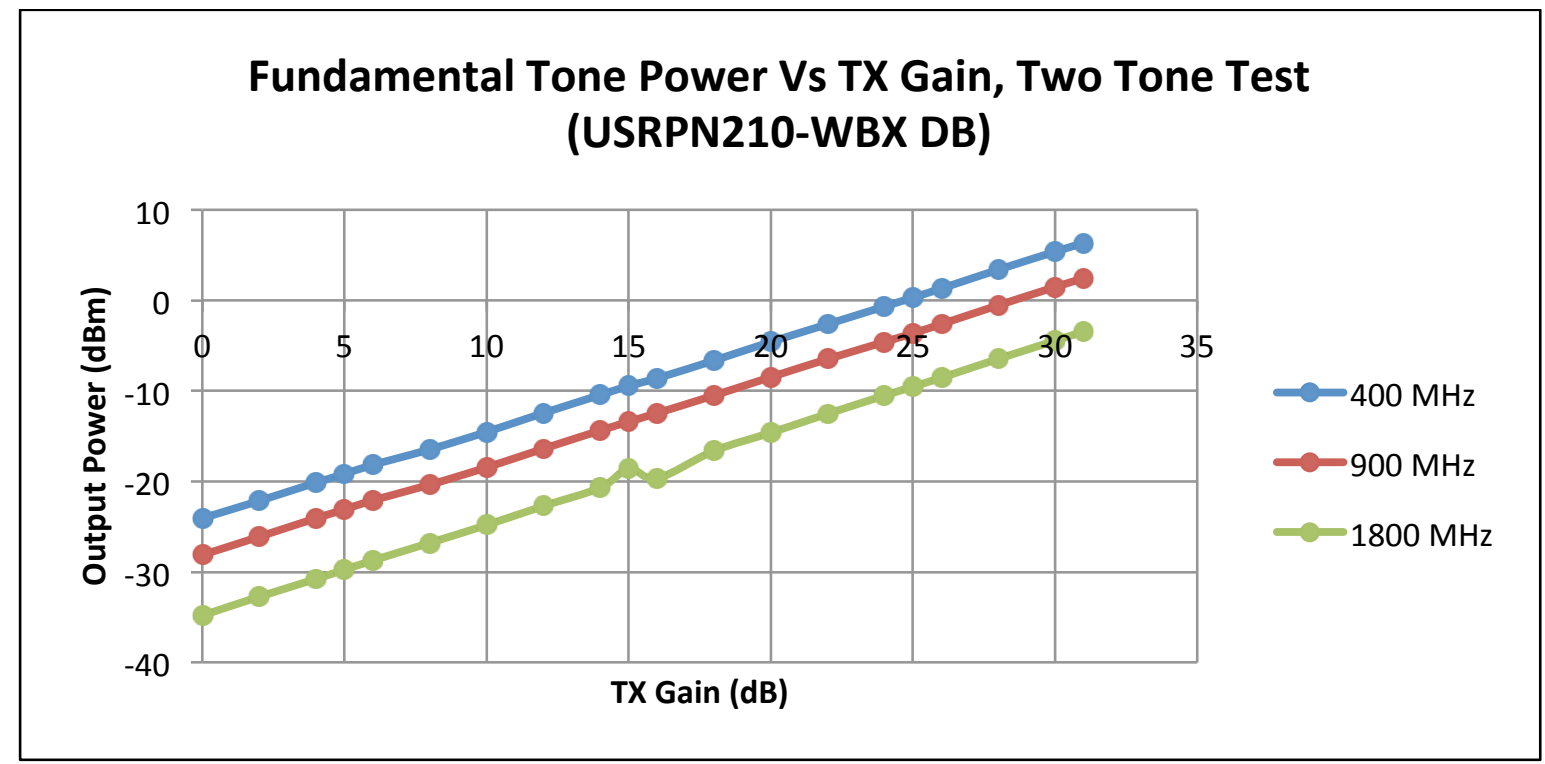

Figure 4.28 F1 Power Variation with TX Gain, Two Tone Test (USRPN210-WBX DB)

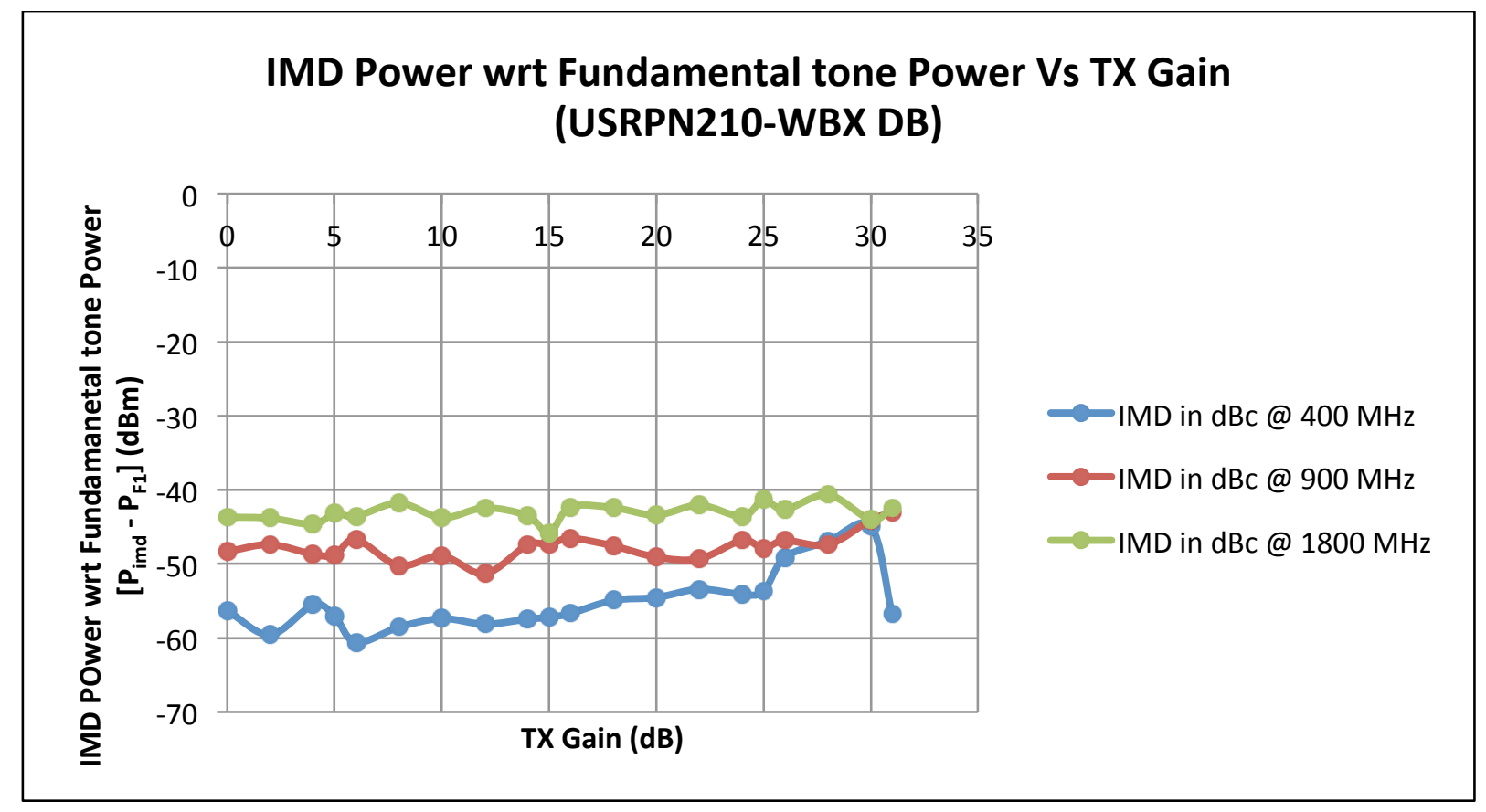

Figure 4.29 Relative IMD Power Variation with TX Gain (USRPN210-WBX DB) 


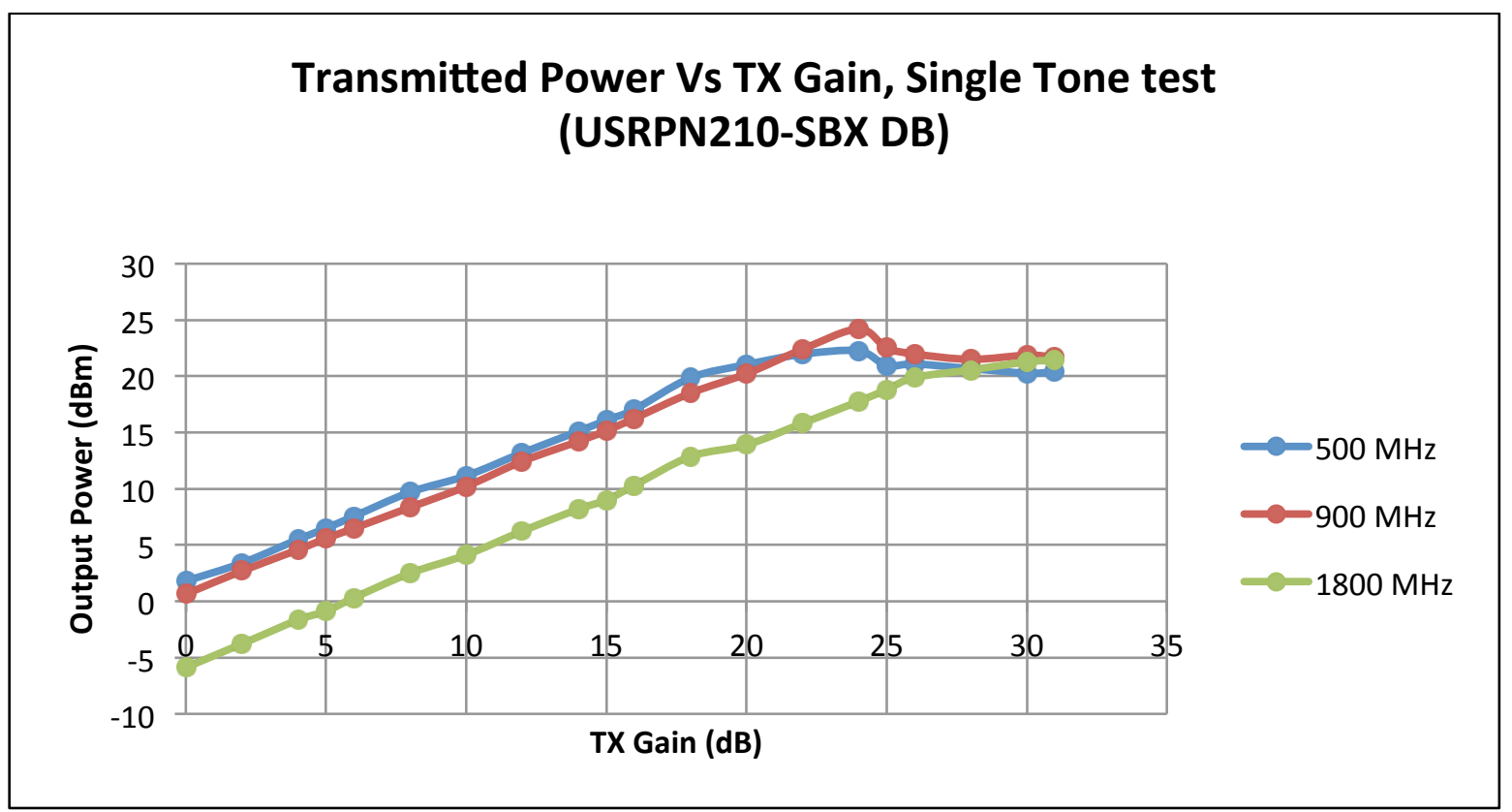

Figure 4.30 Transmitted Power Variation with TX Gain (USRPN210-SBX DB)

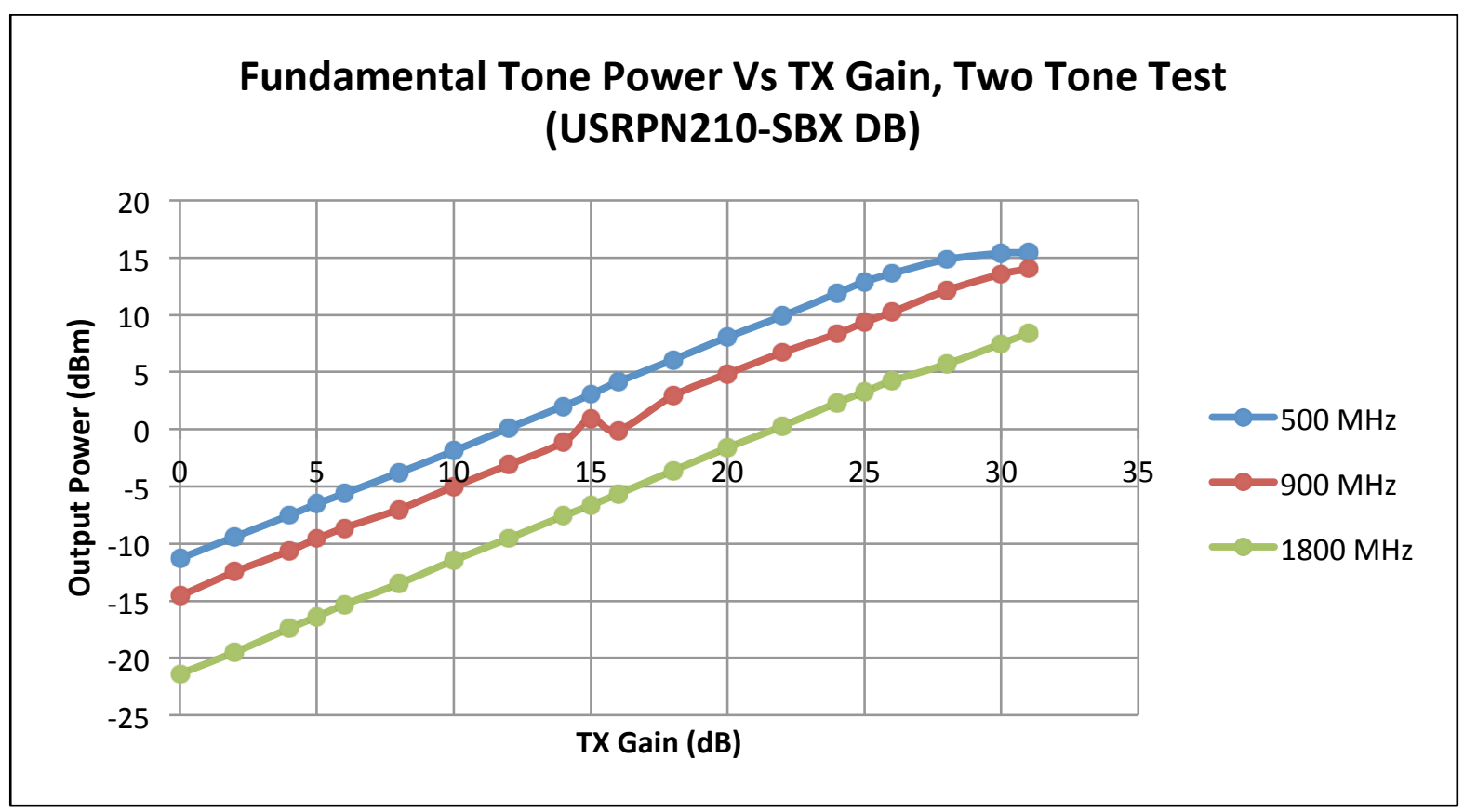

Figure 4.31 F1 Power Variation with TX Gain, Two Tone Test (USRPN210-SBX DB) 


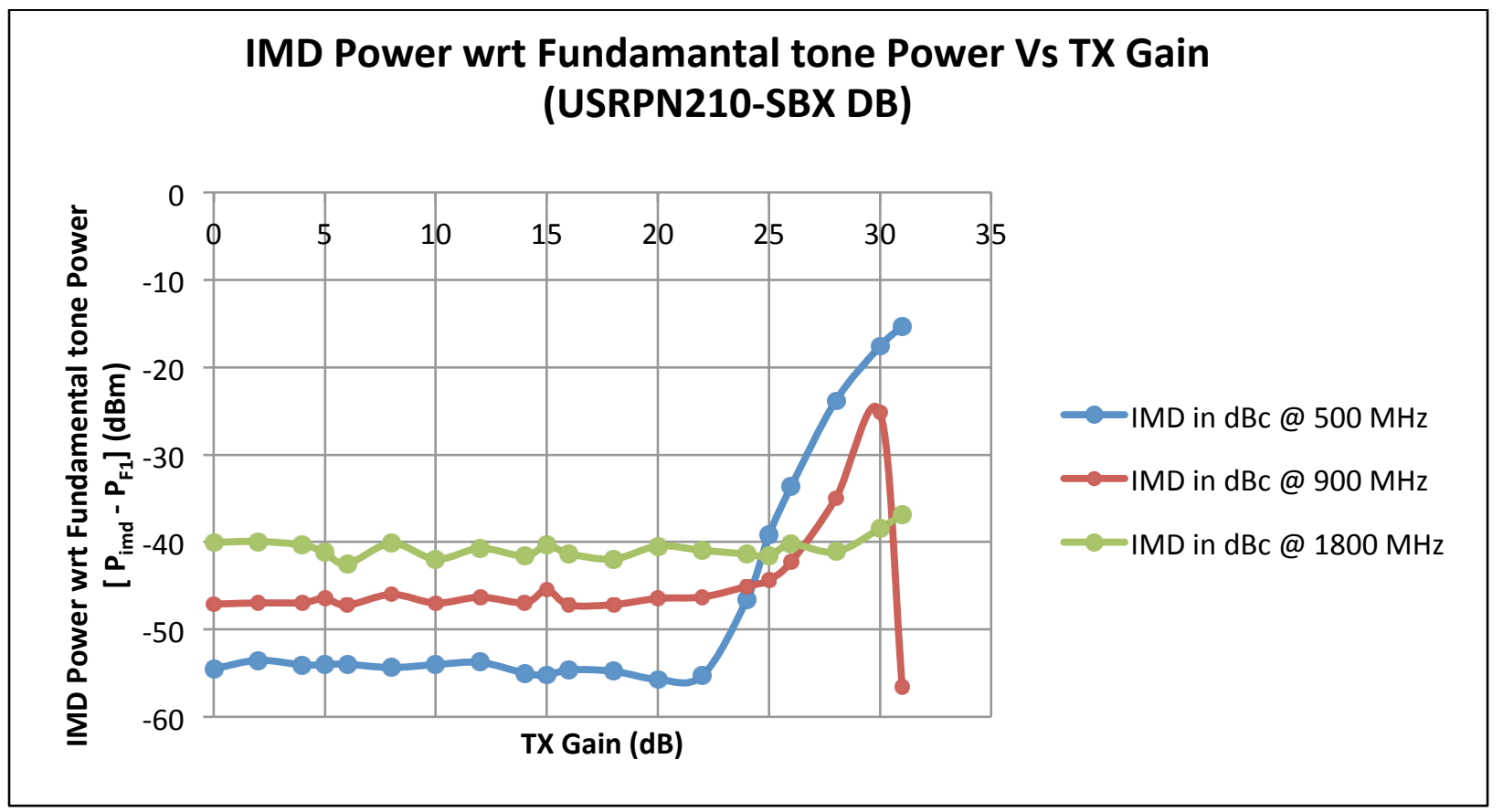

Figure 4.32 Relative IMD Power Variation with TX Gain (USRPN210-SBX DB)

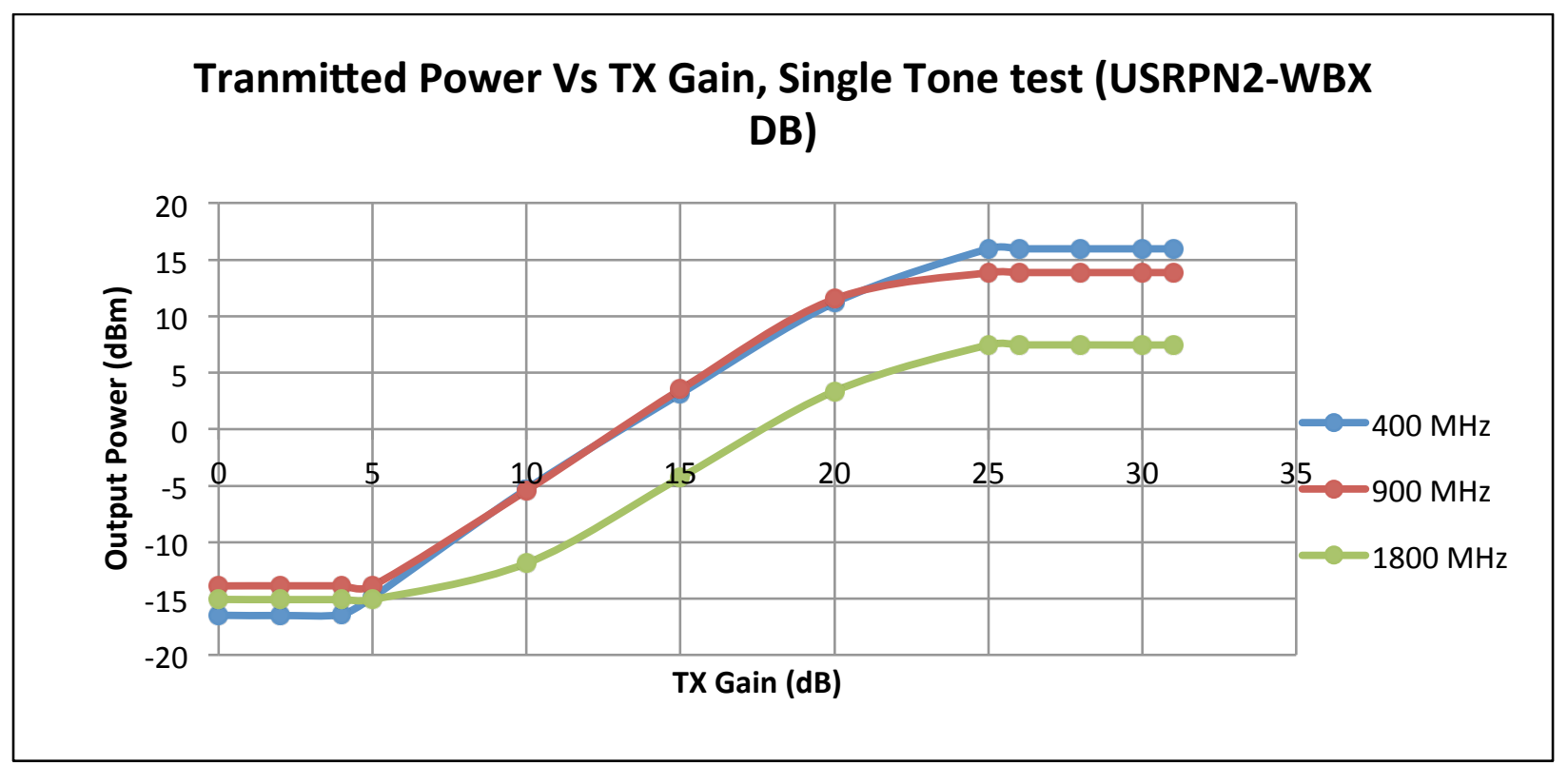

Figure 4.33 Transmitted Power Variation with TX Gain (USRPN2-WBX DB) 


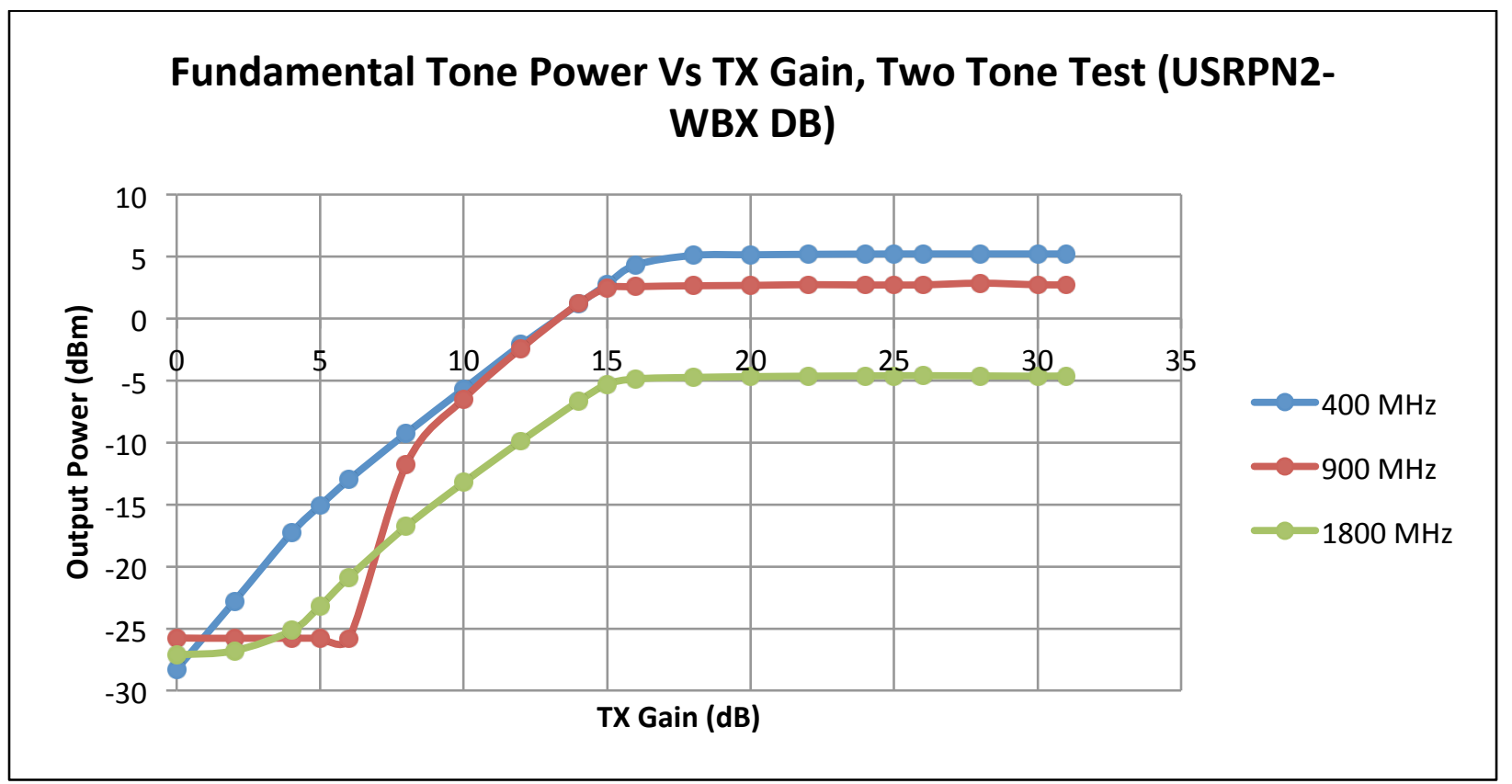

Figure 4.34 F1 Power Variation with TX Gain, Two Tone Test (USRPN2-WBX DB)

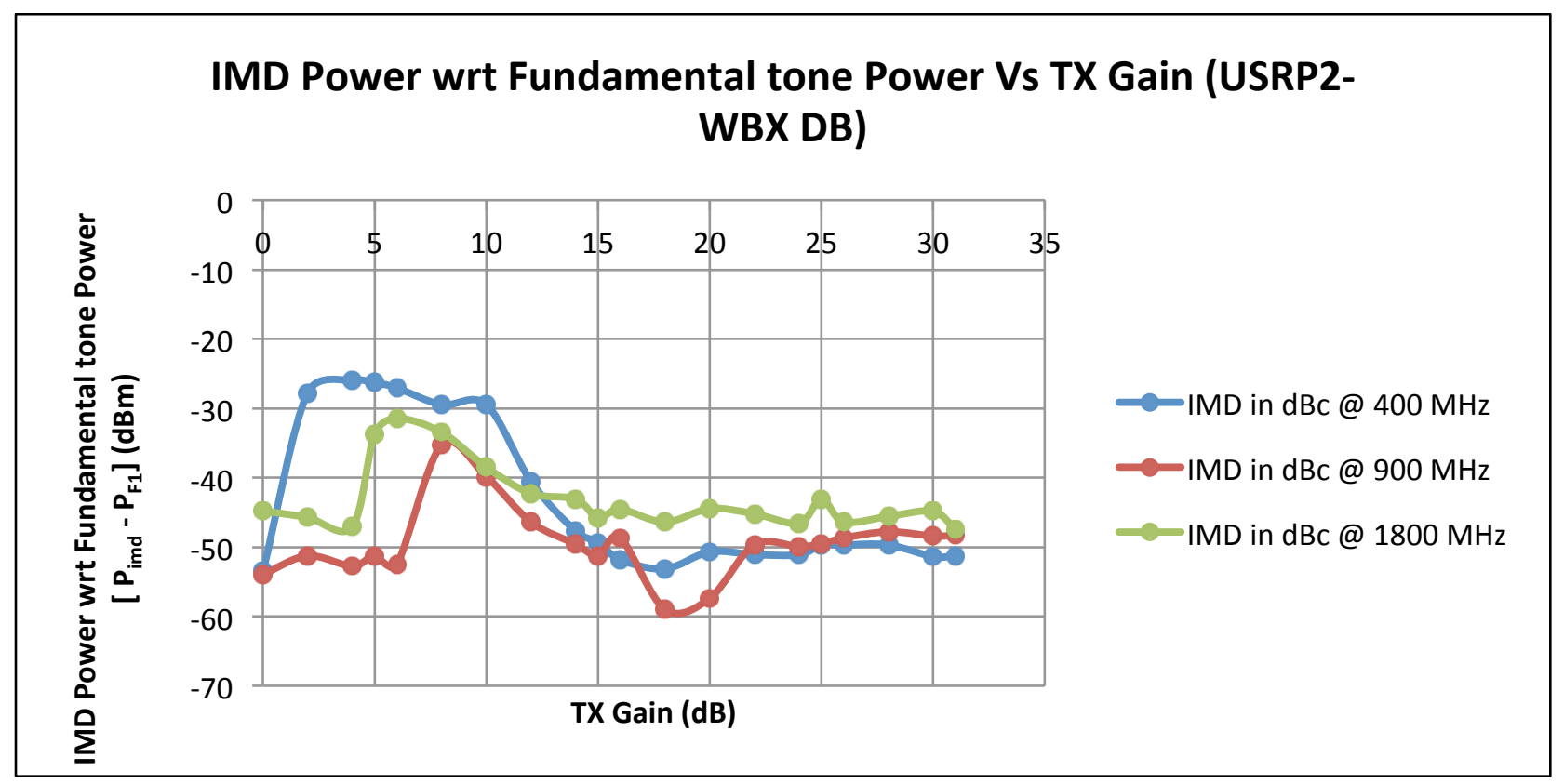

Figure 4.35 Relative IMD Power Variation with TX Gain (USRP2-WBX DB) 
Table 4.11 Power Reference Table: Transmitted Power Variation with TX Gain

\begin{tabular}{|c|c|c|c|c|c|c|c|c|c|}
\hline $\begin{array}{c}\text { Ampl } \\
\text { Variation }\end{array}$ & 0 to 1 & & & & & Test & \multicolumn{3}{|c|}{ Single Tone Tranmitter Power Vs Gain } \\
\hline Frequency & \multicolumn{3}{|c|}{$400 \mathrm{MHz}$} & \multicolumn{3}{|c|}{$900 \mathrm{MHz}$} & \multicolumn{3}{|c|}{$1800 \mathrm{MHz}$} \\
\hline TX GAIN & $\begin{array}{c}\text { DUT :USRPN210 +SBX } \\
\text { Spectrum Analyser } \\
\text { Readings }(\mathrm{dBm})\end{array}$ & $\begin{array}{l}\text { DUT :USRPN210 } \\
\text { +WBX Spectrum } \\
\text { Analyser Readings } \\
\text { (dBm) }\end{array}$ & $\begin{array}{l}\text { DUT :USRP2 +WBX } \\
\text { Spectrum Analyser } \\
\text { Readings (dBm) }\end{array}$ & $\begin{array}{l}\text { DUT :USRPN210 +SBX } \\
\text { Spectrum Ananlyser } \\
\text { Readings (dBm) }\end{array}$ & $\begin{array}{c}\text { DUT :USRPN210+WBX } \\
\text { Spectrum Ananlyser } \\
\text { Readings }(\mathrm{dBm})\end{array}$ & $\begin{array}{c}\text { DUT :USRP2 +WBX } \\
\text { Spectrum Analyser } \\
\text { Readings (dBm) }\end{array}$ & $\begin{array}{c}\text { DUT :USRPN210 +SBX } \\
\text { Spectrum Ananlyser } \\
\text { Readings (dBm) }\end{array}$ & $\begin{array}{c}\text { DUT :USRPN210 +WBX } \\
\text { Spectrum Ananlyser } \\
\text { Readings (dBm) }\end{array}$ & $\begin{array}{c}\text { DUT :USRP2 +WBX } \\
\text { Spectrum Analyser } \\
\text { Readings (dBm) }\end{array}$ \\
\hline \multicolumn{10}{|l|}{ AMPL:1 } \\
\hline 0 & 4.9 & -9.16 & -13.3 & 3.2 & -13.2 & -16.43 & -3.13 & -20.02 & -12.2 \\
\hline 5 & 8.8 & -4.28 & -11.52 & 8.15 & -8.25 & -16.43 & 1.9 & -15.14 & -12.2 \\
\hline 10 & 13.5 & 0.4 & -2.12 & 12.83 & -3.54 & -8.05 & 6.9 & -10.2 & -11.95 \\
\hline 15 & 18.29 & 5.5 & 6.35 & 17.74 & 1.53 & 0.95 & 11.76 & -5.02 & -3.47 \\
\hline 20 & 20.73 & 10.34 & 14.3 & 22.03 & 6.49 & 9.71 & 16.69 & 0 & 4.09 \\
\hline 25 & 20.1 & 14.83 & 15.09 & 22.15 & 11.21 & 13.94 & 20.71 & 5.14 & 10.72 \\
\hline 30 & 19.91 & 17.17 & 15.27 & 21.61 & 14.92 & 14.13 & 21.06 & 10.05 & 10.72 \\
\hline 31 & 20.27 & 17.29 & 15.96 & 21.58 & 15.28 & 13.98 & 20.91 & 10.091 & 10.73 \\
\hline \multicolumn{10}{|l|}{ AMPL: 0.707} \\
\hline 0 & 1.8 & -11.94 & -16.5 & 0.64 & -16.15 & -13.87 & -5.87 & -22.54 & -15.09 \\
\hline 5 & 6.4 & -6.89 & -14.89 & 5.59 & -11.25 & -13.87 & -0.88 & -17.55 & -15.09 \\
\hline 10 & 11.15 & -2.4 & -5.3 & 10.21 & -6.58 & -5.43 & 4.12 & -12.66 & -15.09 \\
\hline 15 & 16.09 & 2.64 & 3.14 & 15.17 & -1.52 & 3.52 & 9 & -7.54 & -11.88 \\
\hline 20 & 20.45 & 7.51 & 11.19 & 20.22 & 3.41 & 11.52 & 13.9 & -2.51 & -4.29 \\
\hline 25 & 20.96 & 12.52 & 15.97 & 22.52 & 8.21 & 13.57 & 18.82 & 2.52 & 3.35 \\
\hline 30 & 20.27 & 17.14 & 15.97 & 21.87 & 13.12 & 13.87 & 21.28 & 7.55 & 8 \\
\hline 31 & 20.39 & 17.65 & 15.97 & 21.69 & 13.95 & 13.87 & 21.43 & 8.52 & 7.99 \\
\hline \multicolumn{10}{|l|}{ AMPL: 0.5} \\
\hline 0 & -1.4 & -15.14 & -19.6 & -2.56 & -19.2 & -10.87 & -9.07 & -26.14 & -17.28 \\
\hline 5 & 3.03 & -10.26 & -17.29 & 2.22 & -14.25 & -10.87 & -4.25 & -21.11 & -17.1 \\
\hline 10 & 7.97 & -5.59 & -7.65 & 7.03 & -9.55 & -2.43 & 0.94 & -16.19 & -11.13 \\
\hline 15 & 12.88 & -0.55 & 0.94 & 11.96 & -4.46 & 6.52 & 5.79 & -11.01 & -3.56 \\
\hline 20 & 17.34 & 4.41 & 10.91 & 17.11 & 0.5 & 14.52 & 10.79 & -5.97 & 3.92 \\
\hline 25 & 21.84 & 9.28 & 16.83 & 21.81 & 5.36 & 16.57 & 15.49 & -0.91 & 5.22 \\
\hline 30 & 20.97 & 14.07 & 16.33 & 21.35 & 10.38 & 16.87 & 20.95 & 4.3 & 5.22 \\
\hline 31 & 20.4 & 14.97 & 16.09 & 20.98 & 11.32 & 16.87 & 20.48 & 5.3 & 5.22 \\
\hline \multicolumn{10}{|l|}{ AMPL: 0.354} \\
\hline 0 & -4.4 & -18.12 & -22.7 & -5.33 & -22.2 & -7.9 & -11.87 & -29.12 & -20.24 \\
\hline 5 & 0.54 & -13.23 & -20.4 & -0.39 & -17.24 & -7.89 & -6.88 & -24.1 & -20.04 \\
\hline 10 & 5.19 & -8.55 & -10.75 & 4.24 & -12.55 & 0.54 & -1.86 & -19.16 & -11.17 \\
\hline 15 & 10 & -3.51 & -2.18 & 9.13 & -7.46 & 9.56 & 3.04 & -13.99 & -3.64 \\
\hline 20 & 15.13 & 1.39 & 7.92 & 14.26 & -2.5 & 17.48 & 8.05 & -8.94 & 2.14 \\
\hline 25 & 19.84 & 6.35 & 13.78 & 18.97 & 2.39 & 17.12 & 13 & -3.89 & 2.3 \\
\hline 30 & 21.1 & 11.41 & 13.23 & 22.35 & 7.46 & 13.39 & 17.19 & 1.23 & 2.3 \\
\hline 31 & 21.03 & 12.23 & 12.99 & 22.43 & 8.45 & 13.13 & 18.06 & 2.36 & 2.4 \\
\hline \multicolumn{10}{|l|}{ AMPL:0 } \\
\hline 0 & -45.46 & -51.54 & -37.25 & -43.93 & -50.15 & -35.46 & -47.93 & -53.15 & -39.19 \\
\hline 5 & -40.93 & -46.51 & -24.5 & -38.98 & -45.25 & -22.15 & -43.06 & -48.22 & -26.25 \\
\hline 10 & -36.24 & -41.81 & -24.15 & -34.97 & -40.55 & -22.99 & -38.05 & -43.25 & -26.11 \\
\hline 15 & -31.46 & -36.81 & -24.05 & -29.52 & -35.48 & -22.4 & -33.33 & -38.08 & -26.06 \\
\hline 20 & -26.48 & -31.92 & -24.19 & -24.47 & -30.51 & -25.18 & -28.43 & -33.03 & -26.02 \\
\hline 25 & -21.46 & -27.05 & -23.56 & -19.72 & -25.65 & -21.34 & -23.47 & -28.01 & -26.1 \\
\hline 30 & -16.61 & -21.89 & -23.93 & -14.68 & -20.53 & -21.76 & -19.24 & -22.82 & -25.95 \\
\hline 31 & -15.53 & -20.93 & -22.67 & -13.71 & -19.52 & -20.55 & -18.34 & -21.8 & -25.07 \\
\hline
\end{tabular}




\subsection{Practical Applications}

The practical applications of the calibration factors was tested using path loss modeling and position estimation tests. The procedures for the same have been detailed below.

\subsubsection{Path Loss Modeling Test}

1. The cornet nodes were used to conduct the Path loss test [12].

2. On the HOST PC, two terminals were opened for remote logging into the CORNET.

3. One of the nodes connected was chosen as the transmitter. The UHD_SIGGEN.py with the following parameters was run at this terminal: -f $460 \mathrm{e} 6,-\mathrm{x}$ 1e3, -s 1e6, amplitude $=0.707$

4. The second terminal was used to ssh into another node, which was the receiver node, which ran the UHD_FFT.grc, flow graph.

5. The center frequency of UHD_FFT was tuned to $460 \mathrm{MHz}$ to avoid phase noise error.

6. The value of power was noted down for this pair of TX-RX.

7. Next the receiver node connection was closed and the terminal was used to ssh into another one of the remaining nodes.

8. The steps from 5 to 7 were repeated. This was done for every working node in the CORNET keeping the transmitter node constant across the floors.

9. For each TX-RX node pair, the path loss was calculated using equation (2.2).

10. Using this path loss value, in equation 2.9, the path loss exponent was calculated using the log-distance path loss formula. PL (@ 1m) was taken to be $25 \mathrm{~dB}$

11. The theoretical path loss was calculated using the ITU model [28] [35] (equation 2.10)

$$
P L=10 * \log (f)+10 * n * \log (d)-28 .
$$


12. Keeping the path loss exponent ' $n$ ' as the unknown, using trial and error different values (starting from 2 to 5) were substituted so as to get the theoretical PL close to practical PL.

13. This was done for the value of the received power from UHD_FFT.gre with and without the correction factor (taken as 35.5 for $450 \mathrm{MHz}$ )

14. It was seen that path loss exponent came to a value $\sim 5$ (close to and as expected from the multi-floor indoor path loss exponent calculated at $914 \mathrm{MHZ}$ and $2.5 \mathrm{GHz}$ ) [10] [23].

15. When received power was used without correction factor, the path loss exponent came to a value between 1 and 2 (for same and one floor above). This is not as per expectations.

16. Hence through this experiment it was seen that calibration factor helps to get values closer to expected real world metrics.

17. The floor attenuation Factor that was calculated as a part of the path loss experiment also gave values similar to ones obtained in [10] [23].

18. The FAF is given by : $F A F=P L \_c-P L$ (same floor); (equation 2.11) where And PL_c is calculated path loss obtained from step 9

PL (same floor) $=$ PL (d0) $+10 * n$ (same floor)* $\log (\mathrm{d})$.

19. Path loss can be calculated at a distance using either multi-floor exponent value or the FAF in an indoor multi-floor environment, E.g.

PL $(30 \mathrm{~m})=25.2+10 * 6.25 * \log (30)=117.5 \mathrm{dBm}$ : multi-floor exponent value used PL $(30 \mathrm{~m})=25.2+10 * 5 * \log (30)+13.88=112.94 \mathrm{dBm}$ : same floor exponent used with FAF (1 floor above) used. 
Table 4.12 Path Loss Calculation with Calibrated Power using CORNET

\begin{tabular}{|c|c|c|c|c|c|c|c|c|c|c|c|c|}
\hline Txd Node & Rxnode & \begin{tabular}{|c} 
Power \\
Transmitted \\
(dBm)
\end{tabular} & $\begin{array}{c}\text { Power } \\
\text { Received on } \\
\text { UHD FT (dB) }\end{array}$ & $\begin{array}{c}\text { RXGain } \\
(A B)\end{array}$ & $\begin{array}{c}\text { Power Received- } \\
\text { Correction Fatcor- } \\
\text { Gain (dBm) }\end{array}$ & $\begin{array}{l}\text { Calculated } \\
P \text { P (dB }\end{array}$ & $20 \log _{10}(1) \mid(\mid \mathrm{dB})$ & $\begin{array}{c}\text { 10nlog(d) } \\
(\mathrm{dB})\end{array}$ & $\begin{array}{l}\text { Kvalue for the } \\
\text { ITUModel|(dB) }\end{array}$ & $\begin{array}{c}\text { Theoretical PL } \\
(\mathrm{dB})\end{array}$ & $\begin{array}{l}\text { Path loss } \\
\text { exponent usint } \\
\text { ITUModel }\end{array}$ & $\begin{array}{l}\text { Path Loss exponent } \\
\text { using log-Distance } \\
\text { Model }\end{array}$ \\
\hline & & & & & & & & & & & & \\
\hline Floor1 & Floor1 & & & & & & & & & & & \\
\hline \multirow[t]{7}{*}{7001} & 7002 & 15.5 & .7 .79 & 15 & $.54,99$ & 70.49 & 53.06 & 45.25 & .28 .00 & 70.31 & 5.37 & 5.37 \\
\hline & 7004 & 15.5 & -35.16 & 15 & .82 .36 & 97.86 & 53.26 & 72.06 & 28.00 & 97.31 & 5.75 & 5.80 \\
\hline & 7005 & 15.5 & 28.00 & 15 & .75 .20 & 90.70 & 53.26 & 65.02 & 28.00 & 90.27 & 4.80 & 4.83 \\
\hline & 7006 & 15.5 & $-37,72$ & 15 & 8492 & 100042 & 53.26 & 755.02 & 28.00 & 100.28 & 5.10 & 5.11 \\
\hline & \begin{tabular}{|l|l|} 
\\
\end{tabular} & 15.5 & -30.56 & 15 & .77.76 & 93.26 & 53.26 & 67.58 & 28.00 & 92.83 & 4.25 & 4.28 \\
\hline & 7011 & 15.5 & 45.51 & 15 & .92 .71 & 108.21 & 53.26 & 822.78 & 28.00 & 108.03 & 4.60 & 4.61 \\
\hline & & \begin{tabular}{|l} 
Averagen \\
(samefloor)
\end{tabular} & & & & & & & & & 4.98 & 5.00 \\
\hline Floor1 & Floor2 & & & & & & & & & & & \\
\hline \multirow[t]{4}{*}{7001} & 7013 & 15.5 & .18 .74 & 15 & .65 .94 & 81.44 & 53.26 & \begin{tabular}{|l|l|}
555.61 \\
\end{tabular} & .28 .00 & 80.87 & 6.60 & 6.67 \\
\hline & 7014 & 15.5 & 28.84 & 15 & .76 .04 & 91.54 & 53.26 & 65.55 & 28.00 & 90.80 & 6.60 & 6.68 \\
\hline & 7024 & 15.5 & 65.59 & 15 & -112.79 & 128.29 & 53.26 & 10238 & 28.00 & 127.64 & 5.55 & 5.59 \\
\hline & & \begin{tabular}{|l|} 
Averagen (1 \\
floor boovele)
\end{tabular} & & & & & & & & & 6.25 & 6.31 \\
\hline Floor1 & Floor3 & & & & & & & & & & & \\
\hline \multirow[t]{3}{*}{7001} & 7035 & 15.5 & .73 .05 & 15 & -120.25 & \begin{tabular}{|l|l}
135.75 \\
\end{tabular} & 53.26 & 110.25 & 28.00 & 135.50 & 6.10 & 6.12 \\
\hline & $\begin{array}{ll}7036 \\
\end{array}$ & 15.5 & .66 .74 & 15 & .113 .94 & 129.44 & 53.26 & 103.46 & 28.00 & 1287.71 & 5.60 & 5.64 \\
\hline & & $\begin{array}{l}\text { Averagen n.2 } \\
\text { floors bovere) }\end{array}$ & & & & & & & & & 5.85 & 5.88 \\
\hline
\end{tabular}


Table 4.13 Path Loss Calculation with Un-calibrated Power using CORNET

\begin{tabular}{|c|c|c|c|c|c|c|c|c|c|c|c|c|}
\hline Ixd Node & Rxnode & $\begin{array}{c}\text { Power } \\
\text { Transmitted } \\
(\mathrm{dBm}) \\
\end{array}$ & $\begin{array}{l}\text { Power Received } \\
\text { on UHD_FT (dB) }\end{array}$ & $\begin{array}{l}\text { RXGain } \\
\text { (AB) }\end{array}$ & $\begin{array}{c}\text { Un Calibrated } \\
\text { Power Received } \\
(A B) \\
\end{array}$ & $\begin{array}{l}\text { Calculated } \\
\text { PL(dBB) }\end{array}$ & $\begin{array}{c}200 \log _{10}(f) \\
(\mathrm{AB})\end{array}$ & \begin{tabular}{|c|} 
10nlog(d) \\
(dB)
\end{tabular} & $\begin{array}{l}\text { Kvalue for the } \\
\text { TTU Model (dB) }\end{array}$ & $\begin{array}{c}\text { Theoretical PL. } \\
\text { (dBm) }\end{array}$ & $\begin{array}{l}\text { Path Loss exponen } \\
\text { using TUM Model }\end{array}$ & \begin{tabular}{|c|} 
Path Loss exponent \\
using log-Distance \\
Model
\end{tabular} \\
\hline Floor1 & Floor1 & & & & & & & & & & & \\
\hline 7001 & 7002 & 15.5 & -7.79 & 15 & $.7,79$ & 23.29 & 53.26 & 8.43 & 28.00 & 33.68 & $N / A$ & $N / A$ \\
\hline & 7004 & 15.5 & -35.16 & 15 & -35.16 & 50.66 & 53.06 & 25.06 & 28.00 & 50.13 & 2.00 & 2.03 \\
\hline & 7005 & 15.5 & -28.00 & 15 & 28.00 & 43.50 & 53.06 & \begin{tabular}{|l|l|}
18.29 \\
\end{tabular} & 28.00 & 43.35 & 1.35 & 1.35 \\
\hline & 7006 & 15.5 & $\begin{array}{l}-377,72 \\
\end{array}$ & 15 & $-37,72$ & 53.22 & 53.06 & 27,95 & 28.00 & 53.01 & 1.90 & 1.90 \\
\hline & 7007 & 15.5 & -30.56 & 15 & -30.56 & 46.06 & 53.06 & 20.67 & 28.00 & 45.73 & 1.30 & 1.31 \\
\hline & 7011 & 15.5 & -45.51 & 15 & -45.51 & 61.01 & 53.06 & 35.81 & .28 .00 & 60.87 & 1.99 & 1.99 \\
\hline & & $\begin{array}{c}\text { Averagen (same } \\
\text { floor) }\end{array}$ & & & & & & & & & 1.71 & 1.72 \\
\hline Floor1 & Floor2 & & & & & & & & & & & \\
\hline 7001 & 7013 & 15.5 & $.18,74$ & 15 & -18.74 & 34.24 & 53.26 & 8.51 & .28 .00 & 33.77 & 1.01 & 1.07 \\
\hline & 7014 & 15.5 & -28.84 & 15 & -28.84 & 44.34 & 53.06 & 18.87 & -28.00 & 43.93 & 1.90 & 1.92 \\
\hline & 7024 & 15.5 & .65 .59 & 15 & -65.59 & 81.09 & 53.06 & \begin{tabular}{|l}
55.71 \\
\end{tabular} & -28.00 & 80.78 & 3.02 & 3.03 \\
\hline & & $\begin{array}{c}\text { Averagen (1 floo } \\
\text { above) }\end{array}$ & & & & & & & & & 1.98 & 2.01 \\
\hline Floor1 & Floor3 & & & & & & & & & & & \\
\hline 7001 & 7035 & 15.5 & .73 .05 & 15 & .73 .05 & 88.55 & 53.06 & 63.26 & 28.00 & 88.32 & 3.50 & 3.50 \\
\hline & 7036 & 15.5 & .66 .74 & 15 & -66.74 & 82.24 & 53.06 & 56.90 & 28.00 & 81.97 & 3.08 & 3.09 \\
\hline & & $\begin{array}{l}\text { Averagen (2 } \\
\text { floors above) }\end{array}$ & & & & & & & & & 3.29 & 3.29 \\
\hline & & & & & & & & & & & & \\
\hline
\end{tabular}


Table 4.14 Floor Attenuation Factor Calculation with Calibrated Power

\begin{tabular}{|c|c|c|c|c|c|c|c|c|}
\hline Txd Node & Rx node & $\begin{array}{l}\text { Power Transmitted } \\
(\mathrm{dBm})\end{array}$ & $\begin{array}{l}\text { Power Received } \\
\text { on UHD_FTT (dB) }\end{array}$ & RX Gain (dB) & $\begin{array}{l}\text { Power Received- } \\
\text { Correction Factor- } \\
\text { Gain }(\mathrm{dBm})\end{array}$ & $\begin{array}{c}\text { Calculated PL } \\
\text { (dB) }\end{array}$ & $\begin{array}{c}\text { FAF Model } \\
{\left[P L(d 0)+10^{*} n(\text { same }\right.} \\
\left.\text { floor })^{*} \log (d)\right](d B)\end{array}$ & $\begin{array}{c}\text { Floor Attenuation } \\
\text { Factor }(\mathrm{dB})\end{array}$ \\
\hline Floor1 & Floor 1 & & & & & & $n$ (same floor) $=5$ & \\
\hline \multirow[t]{7}{*}{7001} & 7002 & 15.5 & -7.79 & 15 & -54.99 & 70.49 & 67.33 & 3.16 \\
\hline & 7004 & 15.5 & -35.16 & 15 & -82.36 & 97.86 & 87.86 & 10.00 \\
\hline & 7005 & 15.5 & -28.00 & 15 & -75.20 & 90.70 & 92.92 & -2.22 \\
\hline & 7006 & 15.5 & -37.72 & 15 & -84.92 & 100.42 & 98.75 & 1.67 \\
\hline & 7007 & 15.5 & -30.56 & 15 & -77.76 & 93.26 & 104.70 & -11.45 \\
\hline & 7011 & 15.5 & -45.51 & 15 & -92.71 & 108.21 & 115.18 & -6.97 \\
\hline & & Average FAF (same floor) & & & & & & -0.97 \\
\hline Floor1 & Floor 2 & & & & & & $n$ (same floor) $=5$ & \\
\hline \multirow[t]{4}{*}{7001} & 7013 & 15.5 & -18.74 & 15 & -65.94 & 81.44 & 67.33 & 14.11 \\
\hline & 7014 & 15.5 & -28.84 & 15 & -76.04 & 91.54 & 74.86 & 16.69 \\
\hline & 7024 & 15.5 & -65.59 & 15 & -112.79 & 128.29 & 117.44 & 10.86 \\
\hline & & $\begin{array}{c}\text { Average FAF (1 floor } \\
\text { above) }\end{array}$ & & & & & & 13.88 \\
\hline Floor1 & Floor 3 & & & & & & $n$ (same floor) $=5$ & \\
\hline \multirow[t]{3}{*}{7001} & 7035 & 15.5 & -73.05 & 15 & -120.25 & 135.75 & 115.57 & 20.19 \\
\hline & 7036 & 15.5 & -66.74 & 15 & -113.94 & 129.44 & 117.57 & 11.86 \\
\hline & & $\begin{array}{c}\text { Average FAF (2 floors } \\
\text { above) }\end{array}$ & & & & & & 16.03 \\
\hline
\end{tabular}

\subsubsection{Position Location Estimation Test}

1. For position location using RSS, 3 nodes were placed at 3 positions around a standardized transmitter (a signal generator). The position coordinates of the transmitter were assumed to be $(0,0)$ and the coordinates of the receiver nodes were calculated w.r.t the $(0,0)$ location $[\mathbf{1 6}][\mathbf{3 6}]$. 
2. The SMA 703 antennas used for the transmitter and receiver nodes. The transmitter was set at $-20 \mathrm{dBm}, 450 \mathrm{MHz}$. Executing the UHD_FFT.grc at each of the node terminals and noting down the readings from this graph at each of the nodes measured the power received at the 3 nodes.

3. The received power value from UHD_FFT was used with and without the correction factor and using the least squares method (Simple RSS based Position Location Algorithm) detailed in section 2, the coordinates of the transmitter were calculated [37] [17].

4. It was seen that the root mean square error value was less when calculated with the calibrated power value than when calculated with the un-calibrated power value, when the experiment was conducted in a semi open area like a big room. This may be attributed to positive multipath interference [38] [17].

5. The room mean square error becomes almost comparable when calculated with calibrated and un-calibrated power values when the experiment is conducted in a corridor. The shadowing and negative multipath interference seem to be more prevalent in this case.

6. This experiment was also conducted with 5 nodes instead of 3 and it was observed that the root mean square error value is lesser when calculated with 5 nodes than when calculated with 3 nodes by almost $40 \%$. Hence the accuracy increases with the number of receiver (reference) nodes used in the calculation. This is in line with the basis of the least mean square algorithm. 
7. Since the UHD_FFT.grc does not give the correct power measurement in $\mathrm{dBm}$, this experiment was also conducted with the flow graph described in Chapter 2 and with Figure 2.4.

8. For this experiment the only change was that instead of UHD_FFT.grc, the power_measue_QT_Sink.grc flow graph was executed at the receiver nodes terminals.

9. It was seen that this flow graph helped get the power in $\mathrm{dBm}$ and hence was more user friendly.

10. The values measured at the receiver nodes in this experiment were used in Simple RSS based Position Location Algorithm and also in Combined Differential RSS Based Position Location Algorithm both detailed in Chapter 2 respectively [30] [16].

11. It was seen that this flow graph was able to provide better results than the UHD_FFT.grc even with and without the respective calibration factors.

12. It was seen that Simple RSS based algorithm was able to estimate the coordinates of the transmitter more accurately and with less error than CDRSS based algorithm with $\mathrm{n}=3.5$ value, though this is not as per expectations. This can be attributed to the positions of the reference nodes and the overall environment of the experimentation.

13. With increasing exponent value it can be seen that error decreases and the difference between the calibrated and un-calibrated RMSE also seems to decrease. This may be due to fact that the circumcenter of the triangle created by the receiver nodes around the transmitter node is shifted closer to actual transmitter position when the exponent value is closer to the actual value. When the path loss exponent is near 3.5 to 4 (' $n$ ' tends to this range of values when calculated using different reference nodes positions assuming a log 
normal distribution for the path loss) the Simple RSS method is seen to give less error and more accurate localization.

14. With respect to calibration, the Simple RSS based method showed considerable difference (RMSE was lesser for calibrated power readings), while the calibration factor had no impact on the CDRSS based method since they are based on differential power measurements. This is also in a way contradicting to the UHD_FFT.grc based measurements and hence it can be seen that the second flow graph is better for such power measurement dependent applications.

15. It was also seen that the between 3 and 5 nodes, the CDRSS based algorithm lent better accuracy to the results and closer $\mathrm{x}$ and $\mathrm{y}$ estimates than simple RSS based algorithm for the same data set of measurements. The error seemed to drop by almost $45 \%$. This was not the case with Simple RSS based algorithm for a set of 5 receiver nodes. The error margin dropped by around $40 \%$.

\subsection{Position Location Experiment Results}

The figure below shows the layout of the room where position location estimation was carried out using UHD_FFT.grc flow graph on the RX nodes for power measurement. 


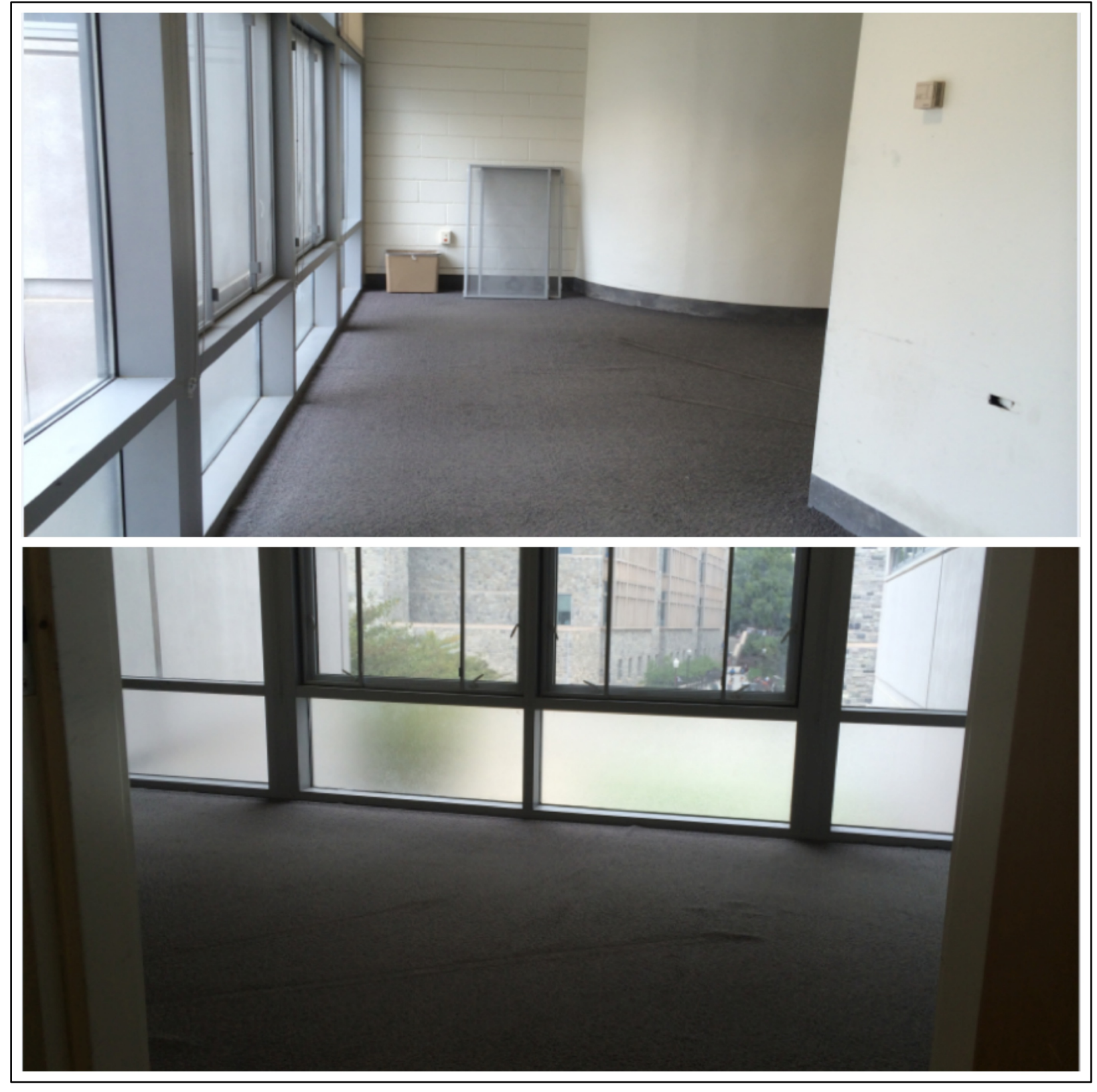

Figure 4.36 Layout of Room Used for Position Location Experiment 


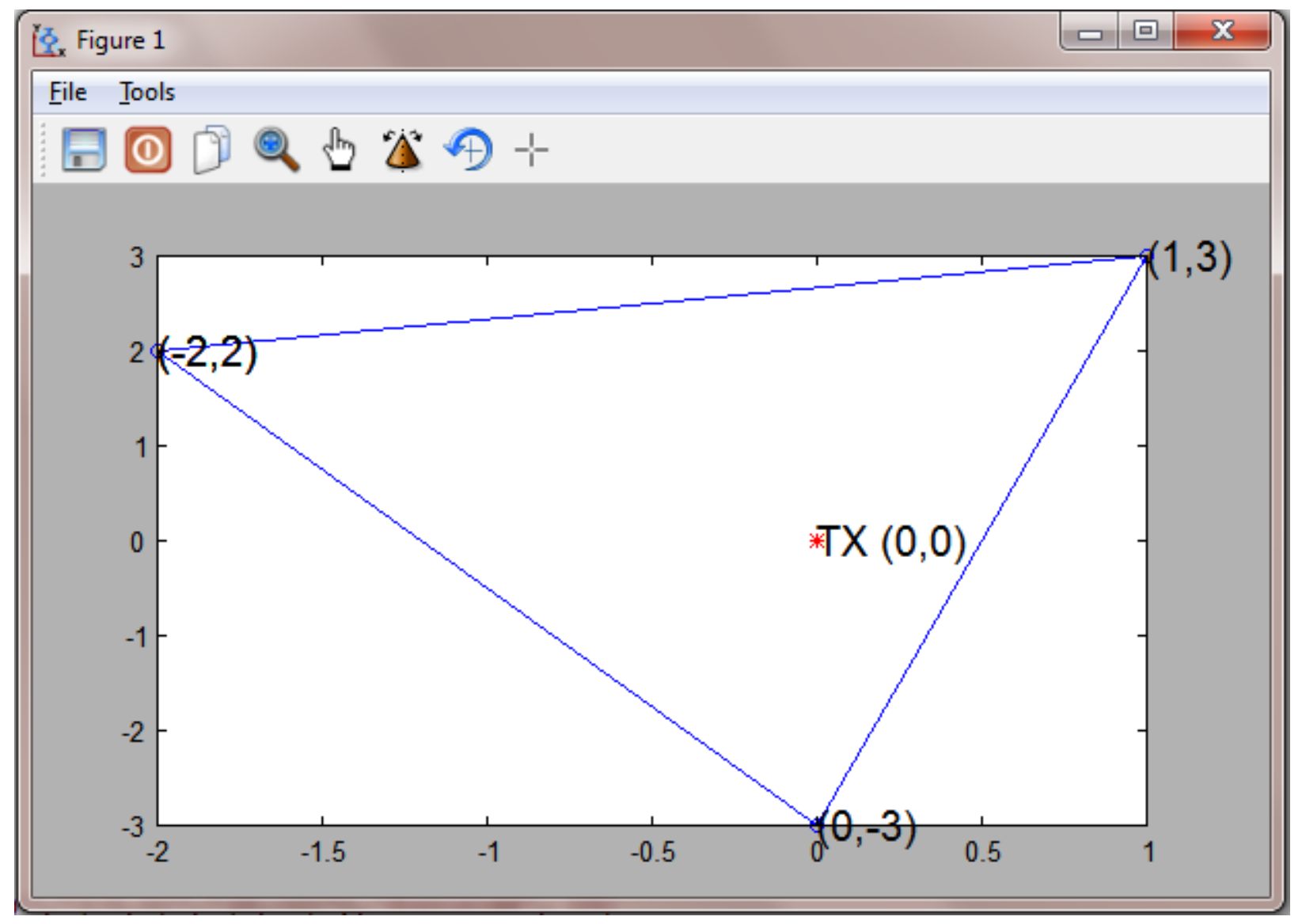

Figure 4.37 Position of the Receiver Nodes w.r.t Transmitter in Large Room

Table 4.15 Root Mean Square Error: Pos. Est. in Large Room

\begin{tabular}{|c|c|c|}
\hline & \multicolumn{2}{|c|}{ Root Mean Square Error } \\
\hline $\begin{array}{c}\text { Number of } \\
\text { nodes/ Power }\end{array}$ & $\begin{array}{c}\text { Calibrated Received } \\
\text { Power (dBm) }\end{array}$ & $\begin{array}{c}\text { Un-Calibrated } \\
\text { Received Power } \\
\text { (dB) }\end{array}$ \\
\hline $\begin{array}{c}\text { 3 Receiver } \\
\text { Nodes }\end{array}$ & 0.34 & 0.44 \\
\hline
\end{tabular}

The figure below shows the layout of the corridor where the position location measurements were carried out using UHD_FFT.grc at the RX nodes. The corridor had multiple pieces of 
furniture and a couple of stairways. Human movement was sparse as the experiment was done during late evening hours.

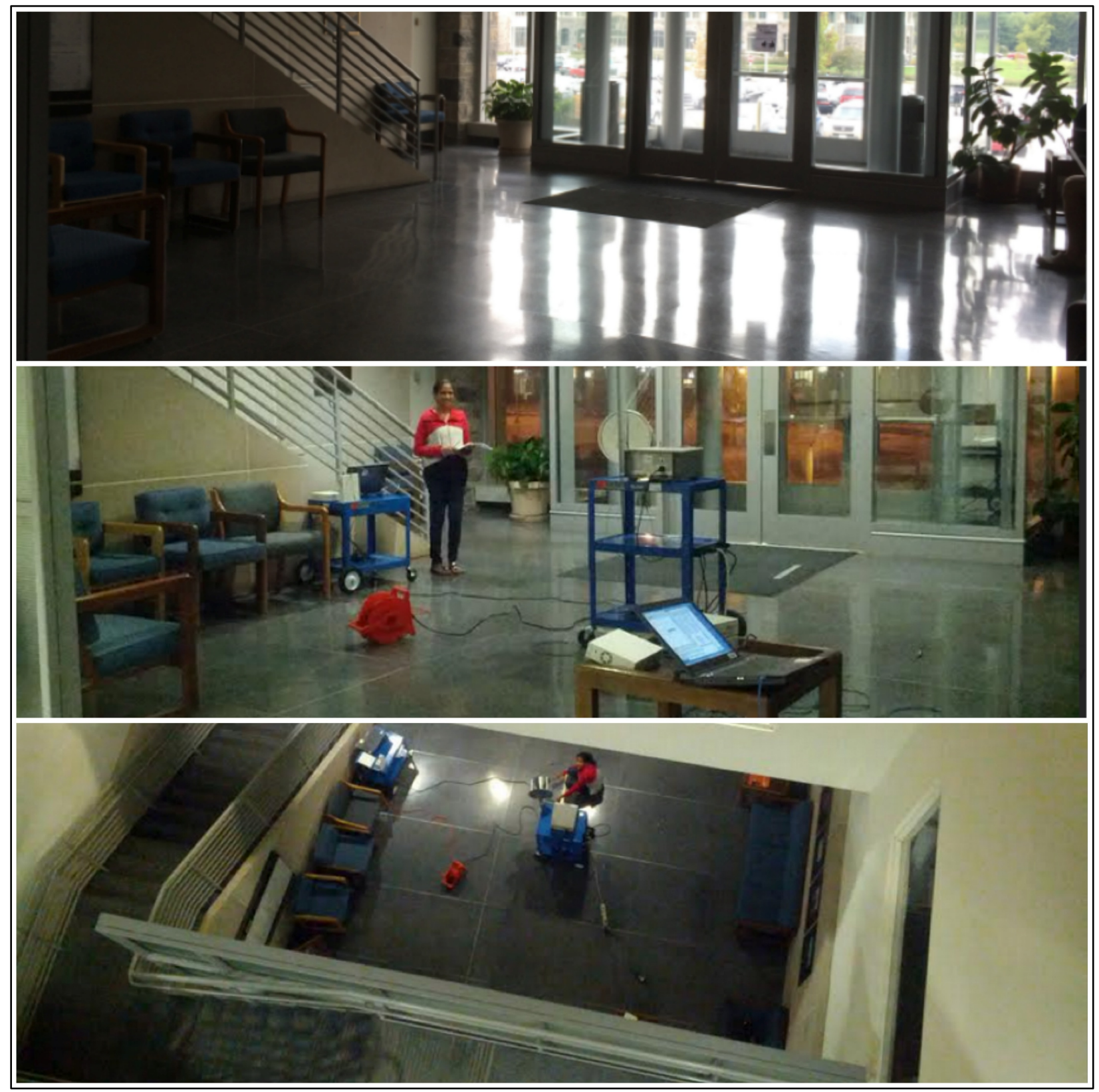

Figure 4.38 Layout of Corridor used for Position Location Experiment 


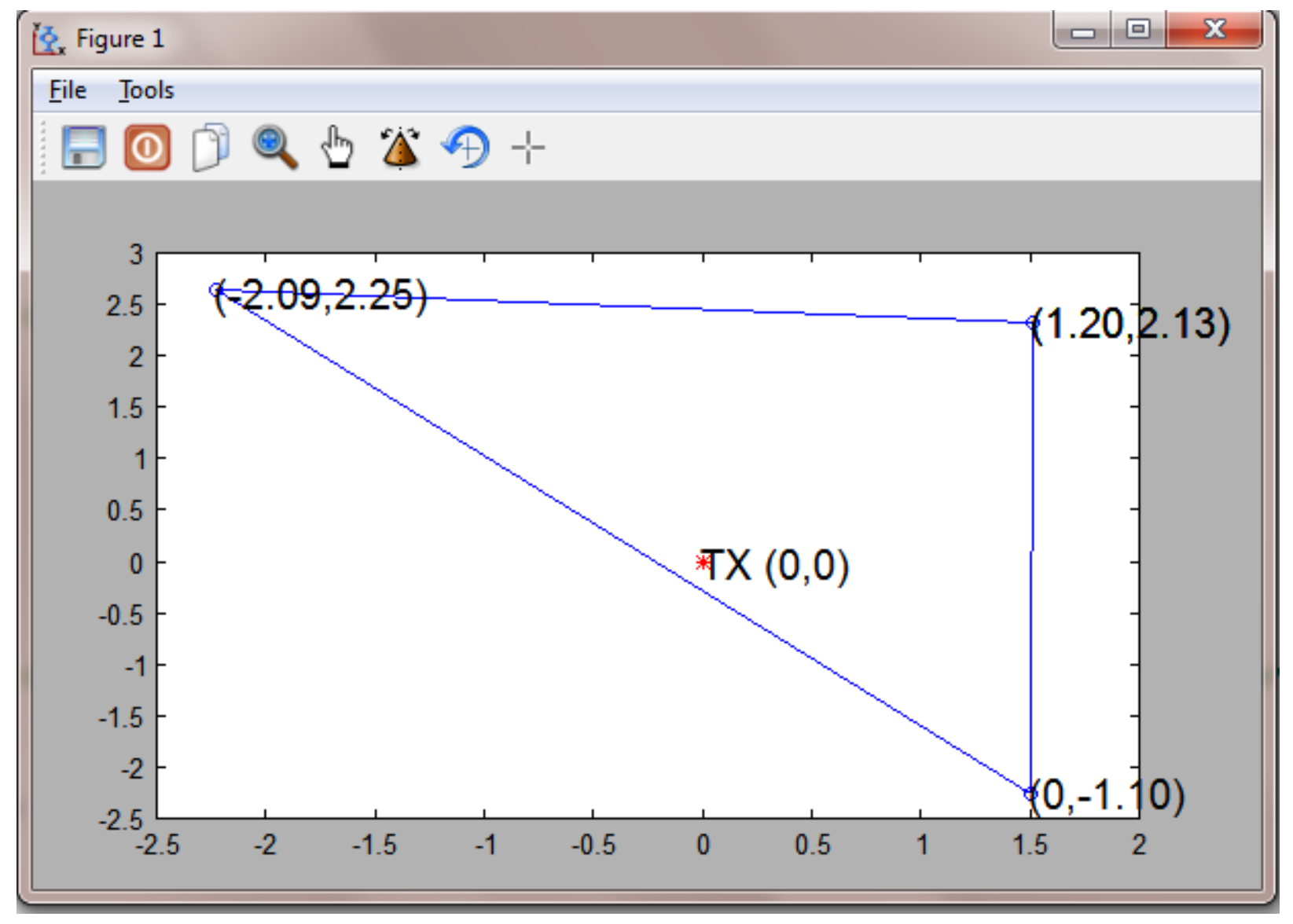

Figure 4.39 Position of Receiver Nodes w.r.t TX in Corridor, Exp 1

Table 4.16 Root Mean Square Error: Pos. Est. in Corridor

\begin{tabular}{|c|c|c|}
\hline & \multicolumn{2}{|c|}{ Root Mean Square Error } \\
\hline $\begin{array}{c}\text { Number of } \\
\text { nodes/ Power }\end{array}$ & $\begin{array}{c}\text { Calibrated Received } \\
\text { Power (dBm) }\end{array}$ & $\begin{array}{c}\text { Un-Calibrated } \\
\text { Received Power } \\
\text { (dB) }\end{array}$ \\
\hline $\begin{array}{c}\text { 3 Receiver } \\
\text { Nodes }\end{array}$ & 0.55 & 0.53 \\
\hline $\begin{array}{c}\text { 5 Receiver } \\
\text { Nodes }\end{array}$ & 0.26 & 0.27 \\
\hline
\end{tabular}

Since UHD_FFT.grc seem to be an efficient power measurement utility, which is a requisite for such RSS, based experiments, a custom flow graph using QT sink block instead was developed 
for more accurate power measurement at the nodes and the position location experiment was repeated.

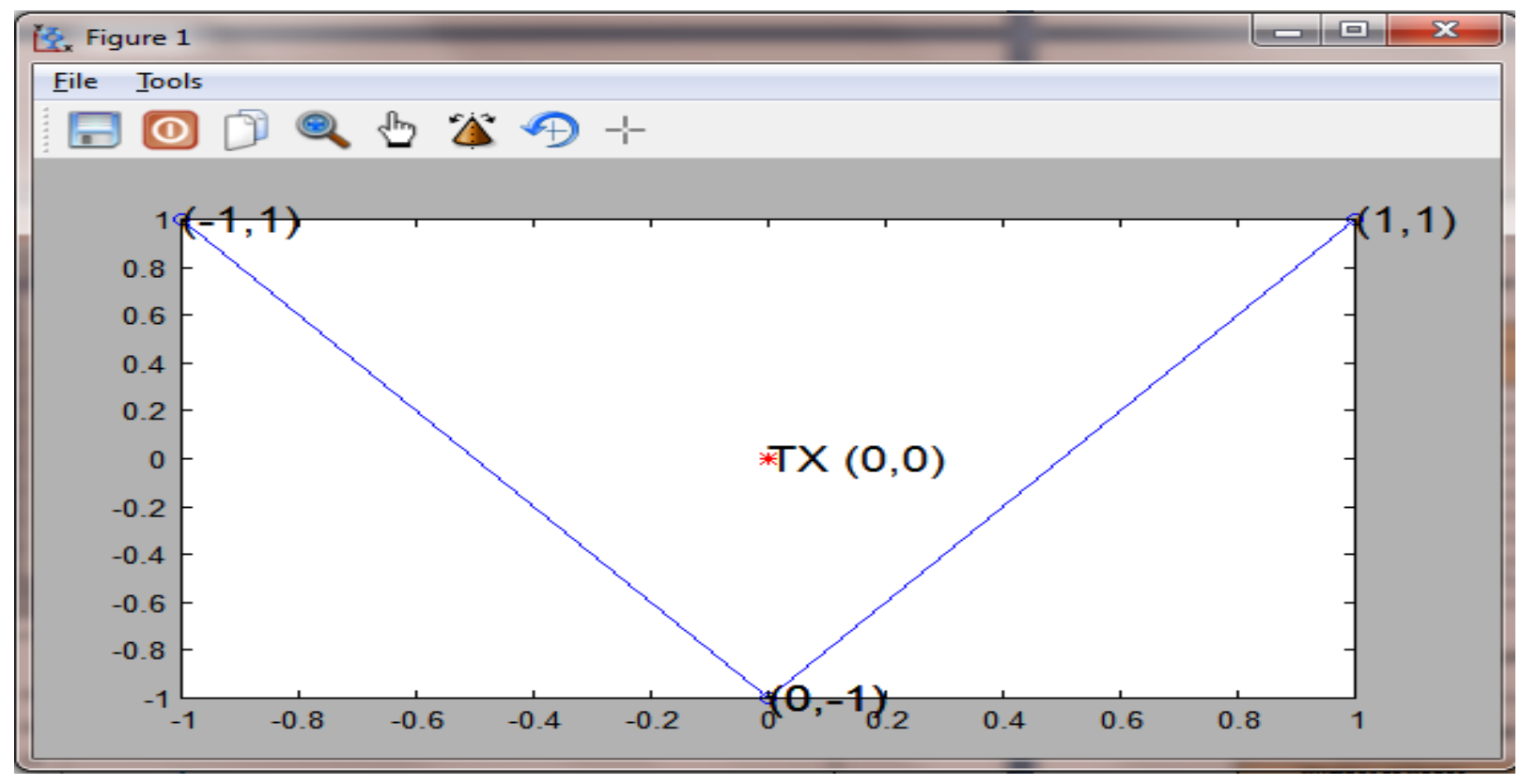

Figure 4.40 Position of Receiver Nodes w.r.t TX in Corridor, Exp 2

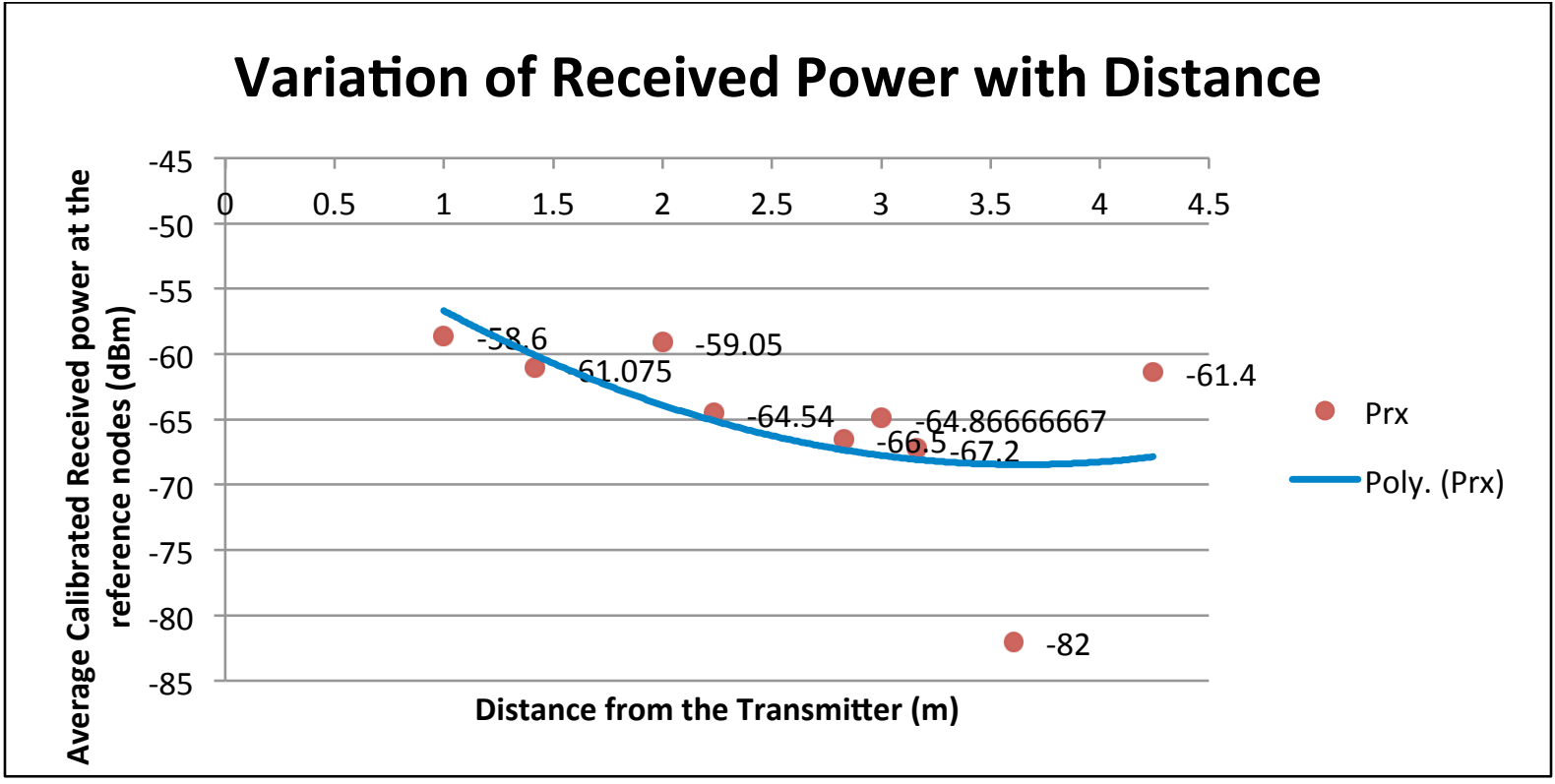

Figure 4.41 Variation of Received Power with Distance 
Table 4.17 Root Mean Square Error: Pos. Est. in Corridor (3 nodes, QT Flow Graph)

\begin{tabular}{|c|c|c|c|c|}
\hline $\begin{array}{c}\text { Using } \\
\begin{array}{c}\text { power_measure_QT_Sink.grc } \\
\text { and } \mathbf{n}=3.5\end{array}\end{array}$ & \multicolumn{2}{c|}{$\begin{array}{c}\text { Simple RSS Based } \\
\text { Algorithm }\end{array}$} & \multicolumn{2}{c|}{ CDRSS Based Algorithm } \\
\hline Number of Nodes & \multicolumn{2}{|c|}{ RMSE (m) } & \multicolumn{2}{c|}{ RMSE (m) } \\
\hline & Calibrated & Un-Calibrated & Calibrated & $\begin{array}{c}\text { Un- } \\
\text { Calibrated }\end{array}$ \\
\hline 3.00 & 0.26 & 0.46 & 0.68 & 0.68 \\
\hline 3.00 & 0.56 & 0.62 & 0.39 & 0.39 \\
\hline 3.00 & 0.72 & 0.78 & 0.51 & 0.51 \\
\hline 3.00 & 0.70 & 0.70 & 1.50 & 1.50 \\
\hline 3.00 & 1.06 & 1.12 & 1.02 & 1.02 \\
\hline & & & & \\
\hline Average & 0.66 & 0.73 & 0.82 & 0.82 \\
\hline Standard Deviation & 0.29 & 0.25 & 0.45 & 0.45 \\
\hline $95 \%$ Confidence Interval & 0.26 & 0.22 & 0.51 & 0.51 \\
\hline & & & & \\
\hline
\end{tabular}

Table 4.18 Root Mean Square Error: Pos. Est. in Corridor (5 nodes, QT Flow Graph)

\begin{tabular}{|c|c|c|c|c|}
\hline $\begin{array}{c}\text { Using } \\
\text { power_measure_QT_Sink.grc } \\
\text { and n=3.5 }\end{array}$ & \multicolumn{2}{|c|}{$\begin{array}{c}\text { Simple RSS Based } \\
\text { Algorithm }\end{array}$} & \multicolumn{2}{c|}{ CDRSS Based Algorithm } \\
\hline & \multicolumn{2}{|c|}{ RMSE (m) } & \multicolumn{2}{c|}{ RMSE (m) } \\
\hline Number of Nodes & Calibrated & Un-Calibrated & Calibrated & $\begin{array}{c}\text { Un- } \\
\text { Calibrated }\end{array}$ \\
\hline 5.00 & 0.54 & 0.57 & 0.23 & 0.23 \\
\hline 5.00 & 0.19 & 0.22 & 0.48 & 0.48 \\
\hline 5.00 & 0.52 & 0.57 & 0.68 & 0.68 \\
\hline & & & & \\
\hline Average & 0.42 & 0.45 & 0.46 & 0.46 \\
\hline Standard Deviation & 0.20 & 0.20 & 0.23 & 0.23 \\
\hline $95 \%$ Confidence Interval & 0.17 & 0.18 & 0.26 & 0.26 \\
\hline
\end{tabular}


Table 4.19 Root Mean Square Error Variation with Path Loss Exponent

\begin{tabular}{|c|c|c|}
\hline $\begin{array}{c}\text { Using power_measure_QT_Sink.grc and number of } \\
\text { nodes = }\end{array}$ & \multicolumn{2}{|c|}{ Simple RSS Based Algorithm } \\
\hline path loss Exponent (n) & \multicolumn{2}{|c|}{ RMSE (m) } \\
\hline 2.00 & Calibrated & Un-Calibrated \\
\hline 2.50 & 0.27 & 0.61 \\
\hline 3.00 & 0.26 & 0.49 \\
\hline 3.50 & 0.26 & 0.46 \\
\hline 4.00 & 0.26 & 0.43 \\
\hline 4.50 & 0.26 & 0.41 \\
\hline & 0.26 & 0.49 \\
\hline Average & & 0.07 \\
\hline Standard Deviation & 0.26 & 0.06 \\
\hline 95 Confidence Interval & 0.00 & 0.00 \\
\hline
\end{tabular}

It is to be noted that the antennas were horizontally polarized for the position location estimation test. As a result, the variations in the received signal strength, in this antenna orientation, were a function of directional antenna pattern as well as distance from the transmitter and location of walls, floors, and objects within the building. If antennas in vertical orientation had been used, it would have probably helped slightly because the pattern of a vertical monopole antenna mounted directly on a USRP would not be omnidirectional either, as the USRP is not a symmetric ground-plane. The pattern of the mono-cone antenna would be approximately omnidirectional in the horizontal plane if the antennas were vertically oriented i.e. ground plane is horizontal and the mono-cone antenna is above the ground plane. 


\section{CONCLUSION}

The Universal Software Radio Peripheral, USRP, a RF front-end device catering to software defined radio researchers and hobbyists, is made to be cost effective and user-friendly. These devices are also highly dependent on the software interface and system capabilities. Many times USRP's are viewed as black box devices that allow multiple operations with ease.

One of the major drawbacks seen for such devices is the inability to relate to real-world metrics and hence restricting their practical applications in experiments requiring highly calibrated devices. Experiments for position location estimation for example require nodes that would be able to give precise received power so as to be able to model the path loss and also reduce the error in location estimation.

Using the USRP devices, various experiments were performed which helped analyze and understand the performance of this device under different conditions. One of the main drawbacks for any laboratory setup would be an un-calibrated device, and here through the various tests performed, we were able to obtain calibration factors, which would help in achieving results very close to the near expected results. Some experiments performed like the Received Power stability test in fact reinstated the values found for the calibration factors, and also showed that USRP tends to show a decrement in the received power as the frequency increased. This may be attributed to the fact that the received signal within the USRP device undergoes many stages of processing like analog to digital conversion, decimation, amplification and these stages may themselves require the signal to be normalized within its operational power and frequency range. The UHD_FFT.py itself is a software program that enables a spectrum analyzer GUI and hence 
would not be displaying the real power levels. This would ultimately affect the output seen on UHD_FFT.py GUI.

The transmitter side tests also helped evaluate the output power levels transmitted, to a certain degree of accuracy for various amplitude and gain values set in the UHD_SIGGEN.py program. It has been difficult to predict effectiveness of results obtained when USRP is used as a transmitter as the UHD_SIGGEN software utility also performs some operations on normalized values. Hence an attempt was been made to tabulate the output power levels for different amplitude and gain combinations, which would provide reference for future experiments.

The calibration factors obtained through these experiments were used on practical applications like multi-floor path loss exponent determination and Position location. It was evident that with calibration, the results obtained were closer to the expected values, reducing the mean square error from 0.72 to 0.66 .

Another experiment that helped verify this was the IIP3 test for USRPN210. The IIP3 value obtained without the applied correction factor showed almost a $30 \mathrm{~dB}$ deviation from the expected value while the IIP3 value with the correction factor applied showed a deviation of around $1 \sim 2 \mathrm{~dB}$.

Though lot of research is ongoing in field of software-defined radios, these handy USRP devices are still unexplored in terms of its performance abilities. Calibration is just one step closer to getting these devices a wider platform for real world applications. A lot of documentation in terms of useful reference metrics is lacking and experiments that explore the performance abilities would help provide a good reference for researchers. 
Another important aspect that was noted is that the CORNET test-bed housed in Virginia Tech's Kelly Hall has many educational and research purposes. But again, reference materials, that provide a model for channel propagation characteristics that occur when these nodes are used, would increase utility towards practical applications. Hence, more attention in this area would help develop their practical operability. 


\section{BIBLIOGRAPHY}

[1] Jean-Philippe Lang. (2013) GNU Radio - The Free and Open Software Radio Ecosystem. [Online]. http://gnuradio.org/redmine/projects/gnuradio/wiki/TutorialsCoreConcepts

[2] Jean-Philippe Lang. (2013) GNU Radio - The Free and Open Software Ecosystem. [Online]. http://www.gnu.org/software/gnuradio

[3] Scott Johnston. (2011, October) Boston University Website - MIT Lincoln Laboratory. [Online]. http://people.bu.edu/mrahaim/NEWSDR/Presentations/NEWSDR_Johnston.pdf

[4] Michael Hills, "Developing a Generic Software Defined Radar Transmiter using GNU Radio," School of Electrical and Electronics Engineering, The University of Adelaide, Adelaide, M.S Thesis November 2012.

[5] Jean-Philippe Lang. (2012) Ettus Research LLC Website. [Online]. http://code.ettus.com/redmine/ettus/projects/uhd/wiki/GNU_Radio_UHD

[6] Terrence J. Brisebois, "Wideband RF Front End Daughterboard Based on the Motorola RFIC," Electrical Engineering, Virginia Tech, Blacksburg, VA, M.S Thesis 2009.

[7] (2014) Ettus Research - A National Instruments Company. [Online]. https://www.ettus.com/product

[8] (2014) Ettus Research - A National Instruments Company. [Online]. https://www.ettus.com/content/files/07495_Ettus_N200-210_DS_Flyer_HR_1.pdf

[9] (2014) Ettus Research - A National Instruments Company. [Online]. https://www.ettus.com/product/category/Daughterboards

[10] Scott Y Seidel and Theodore S Rappaport, "914 MHz Path Loss Prediction Models for 
Indoor Wireless Communications in Multifloored Buildings," IEEE Transactions on Antennas and Propagation, vol. 40, no. 2, February 1992.

[11] Theodore S. Rappaport, Wireless Communications: Principles and Practice, 2nd ed.: Prentice Hall, 2002.

[12] (2010) CORNET - Cognitive Radio Network Testbed. [Online]. http://cornet.wireless.vt.edu/

[13] (2011, September) University of California at Los Angeles. [Online]. $\underline{\text { http://cores.ee.ucla.edu/index.php?title=Cognitive_Radio_Testbeds }}$

[14] Nicholas Polys, Peter Sforza Nikita Sharakhov, "GeoSpy: a Web3D platform for geospatial visualization," in 1st ACM SIGSPATIAL International Workshop on MapInteraction MapInteract'13, New York NY, 2013, pp. 30-35.

[15] Jean-Philippe Lang. (2013) GNU Radio - The Free and Open Software Radio Ecosystem. [Online]. http://gnuradio.org/redmine/projects/gnuradio/wiki/FAQ\#How-do-I-know-theexact-voltagepower-of-my-received-input-signal

[16] Ayad M. H. Khalel, "Position Location Techniques in Wireless Communication Systems," Department of Electrical Engineering, Blekinge Institute of Technology, Karlskrona, SWEDEN, M.S Thesis 2010.

[17] J.R. Casar, P. Tarrio A.M. Bernardos, "Real time calibration for RSS indoor positioning systems," in Indoor Positioning and Indoor Navigation (IPIN), 2010.

[18] (2014, August) GNU Radio - The Free \& Open Software Radio Ecosystem. [Online]. http://gnuradio.org/doc/doxygen/group_measurement_tools_blk.html\#ga56518da55fdaa 
3723f89e20f2db2ca12

[19] (2014, July) Ettus Research - A National Instruments Company. [Online]. http://files.ettus.com/manual/page_calibration.html\#calibration

[20] (2014) Ettus Research - A National Instruments Company. [Online]. http://www.ettusresearch.com/content/files/kb/application_note_uhd_examples.pdf

[21] (2014) GNU Radio Archive. [Online]. http://lists.gnu.org/archive/html/discussgnuradio/2010-11/msg00542.html

[22] Arvind Chandrasekaran, "Intra-Vehicle Channel Characterization in the $5 \mathrm{GHz}$ Band," Department of Electrical and Computer Science, Ohio University, Athens OH, M.S Thesis 2011.

[23] T. R. Sontakke B. R. Jadhavar, "2.4 GHz Propagation Prediction Models for Indoor Wireless Communications Within Building," International Journal of Soft Computing and Engineering (IJSCE), vol. 2, no. 3, July 2012.

[24] John S. Seybold, Introduction to RF Propagation. Hoboken, NJ, USA: John Wiley \& Sons Inc., 2005.

[25] Olivera Notaros, Nishant Jadhav Timothy X Brown. (2014) Keysight Technologies. [Online]. http://www.keysight.com/upload/cmc upload/All/exp94e.pdf?\&cc=US\&lc=eng

[26] University of Vigo. [Online]. http://webs.uvigo.es/servicios/biblioteca/uit/rec/P/R-RECP.1411-4-200708-I!PDF-E.pdf

[27] (2014, August) Wikipedia: A Non-Profit Organization. [Online]. http://en.wikipedia.org/wiki/Least_squares 
[28] Balakrishnan Ramasankar, "Comparison of Radio Propagation Models for WiMax Coverage at $450 \mathrm{MHz}, 2.5 \mathrm{GHz}$ and $3.5 \mathrm{GHz}$ for Different Terrains," Department of Electrical Engineering, Glasgow Caledonian University, Glasgow, Scotland, M.S Thesis 2012.

[29] (2014, August) Wikipedia - $\quad$ The free encyclopedia. [Online]. http://en.wikipedia.org/wiki/Total_least_squares

[30] Islam Alyafawi, Desislava Dimitrova, and Torsten Ingo Braun, "SDR-based Passive Indoor Localization System for GSM," in SIGCOMM Software Radio Implementation Forum (SRIF), Chicago IL, 2014.

[31] Lou Frenzel. (2013, October) Electronic Design. [Online]. http://electronicdesign.com/what-s-difference-between/what-s-difference-between-thirdorder-intercept-and-1-db-compression-point

[32] (2009) Signal Processing Group Inc., Website - Wireless and Analog ASICs and Modules. [Online]. http://www.signalpro.biz/pointsf1.pdf

[33] William Domino and Nooshin Vakilian Darioush Agahi. (2002, March) Microwave Journal. [Online]. $\quad$ http://www.microwavejournal.com/articles/3411-two-tone-vs-single-tonemeasurement-of-second-order-nonlinearity

[34] (2012, February) National Instruments Corporation. [Online]. http://www.ni.com/whitepaper/2952/en/

[35] (2014) ITU - Committed to Connecting The World. [Online]. https://www.itu.int/dms_pubrec/itu-r/rec/p/R-REC-P.525-2-199408-I!PDF-E.pdf 
[36] Yung-Fa Huang and You-Ting Jheng. Chaoyang University of Technology. [Online]. http://ir.lib.cyut.edu.tw:8080/bitstream/310901800/12095/1/C08.pdf

[37] Songfeng Zheng. (2010) Missouri State University. [Online]. http://people.missouristate.edu/songfengzheng/Teaching/MTH541/Lecture\%20notes/evalua tion.pdf

[38] (2014, May) Wikipedia - The free encyclopedia. [Online]. http://en.wikipedia.org/wiki/Mean_squared_error

[39] (2014) QSL.net - Connecting Hams Around The World. [Online]. http://www.qsl.net/pa2ohh/jsffield.htm 


\section{APPENDIX A. Position Location Simulation}

\% To find the Simple RSS Based position location using 3 nodes, location: 3rd floor Durham

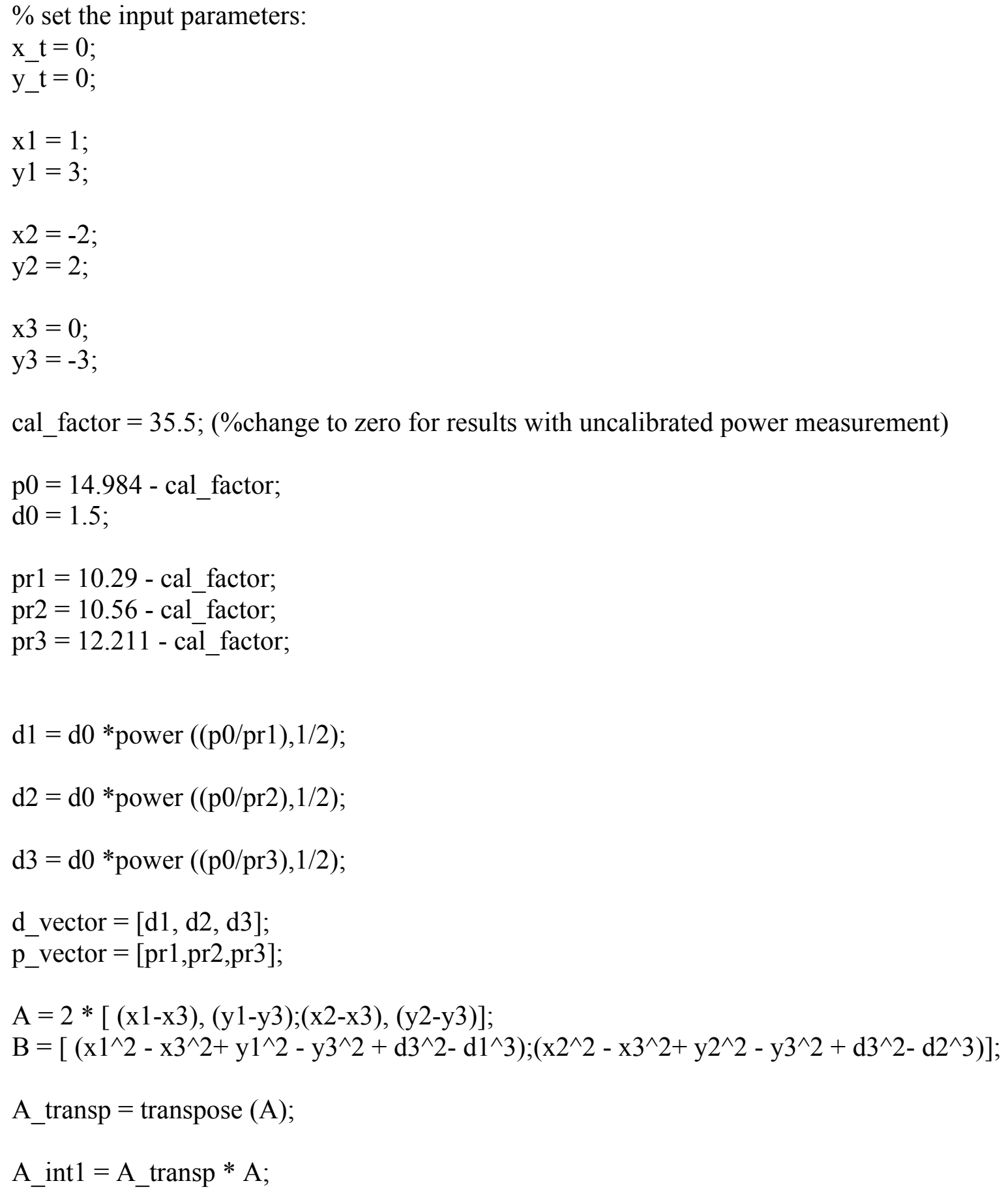




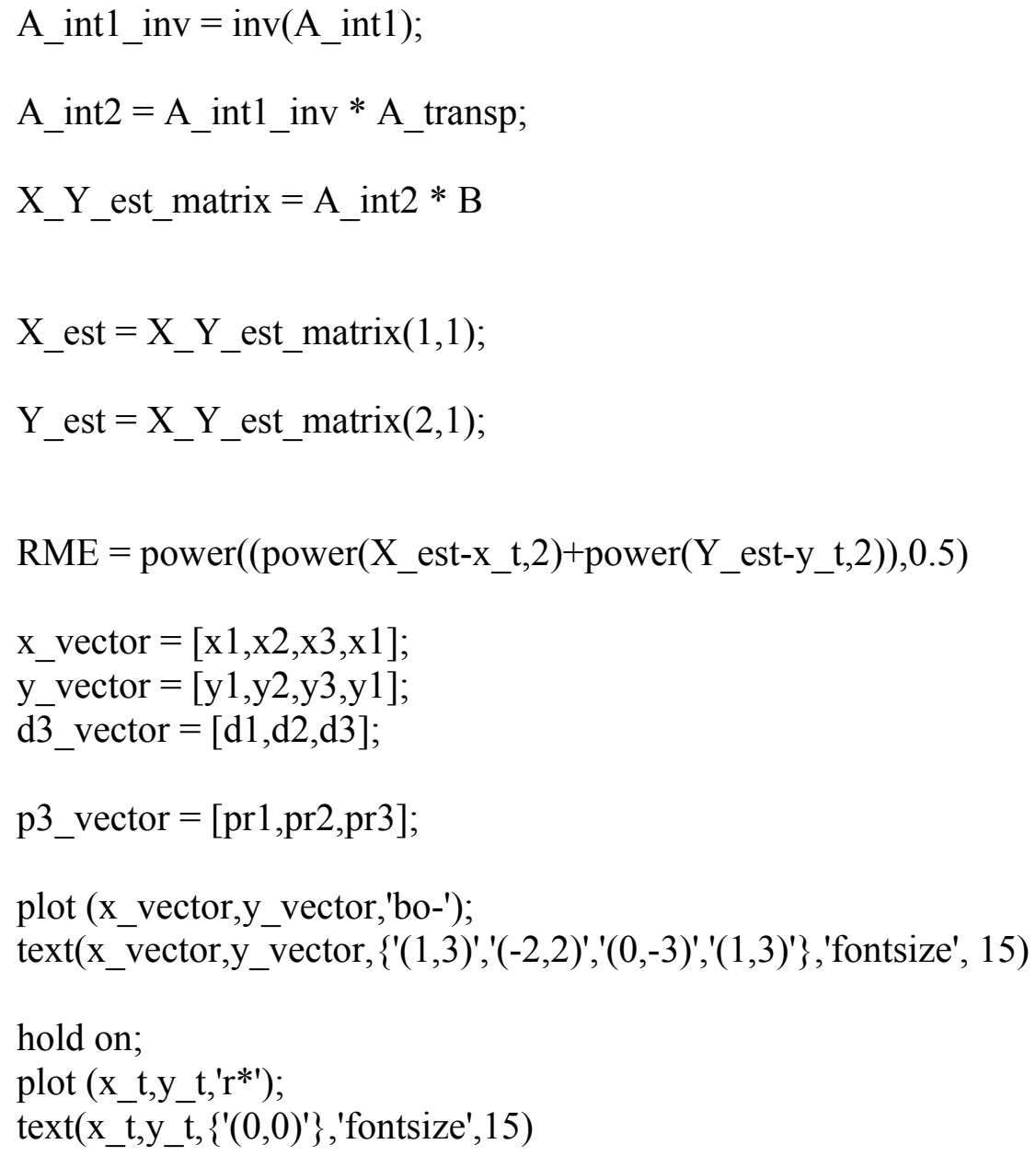


\% To find the CDRSS Based position location using 5 RX nodes, Location : 1st Floor Durham

$\%$ set the input parameters:

clc;

$\mathrm{x} \_\mathrm{t}=0$;

$\mathrm{y} \_\mathrm{t}=0$

$\mathrm{x}=[0,1,1,-1,-1 ; 0,0,0,1,-1 ;-2,1,-2,1,-2]$;

$\mathrm{y}=[-1,2,1,1,2 ; 1,-1,-1,2,1 ;-1,-2,1,3,3]$;

$\operatorname{Pr}=[-1,-4.3,-4.5,-5,-16 ; 5,1.5,-1.4,-4.5,-5 ; 2,-1.4,-3,-14,-22]$;

rows $=\mathrm{s}(1)$

$\operatorname{cols}=\mathrm{s}(2)$

$\mathrm{X}$ est $=$ zeros $(1$, rows $)$;

Y_est $=$ zeros $(1$, rows $)$;

RMSE $=$ zeros $(1$, rows $)$;

dist vect $=$ zeros $($ rows, 5$)$;

final_matrix_cal = zeros (rows, 2);

d_avg $=$ zeros $(1$, rows $)$;

p_vect $=$ zeros $($ rows, 5$)$;

cal_factor $=60 ; \%$ (approx. cal factor @ $450 \mathrm{MHz})$

fori $=1$ :rows,

count $=\mathrm{i}$;

$\operatorname{dr} 1=\operatorname{sqrt}\left(\operatorname{power}\left(\left(x(i, 1)-x_{-} t\right), 2\right)+\operatorname{power}\left(\left(y(i, 1)-y_{-} t\right), 2\right)\right)$;

dr2 $=\operatorname{sqrt}\left(\operatorname{power}\left(\left(x(\mathrm{i}, 2)-\mathrm{x}_{-} \mathrm{t}\right), 2\right)+\operatorname{power}\left(\left(\mathrm{y}(\mathrm{i}, 2)-\mathrm{y}_{-} \mathrm{t}\right), 2\right)\right)$;

dr3 = sqrt $\left(\operatorname{power}\left(\left(x(i, 3)-x_{-} t\right), 2\right)+\operatorname{power}\left(\left(y(i, 3)-y_{-} t\right), 2\right)\right)$;

$\operatorname{dr} 4=\operatorname{sqrt}\left(\right.$ power $\left.\left(\left(x(i, 4)-\mathrm{x} \_\mathrm{t}\right), 2\right)+\operatorname{power}\left(\left(\mathrm{y}(\mathrm{i}, 4)-\mathrm{y} \_\mathrm{t}\right), 2\right)\right)$;

$\operatorname{dr} 5=\operatorname{sqrt}\left(\right.$ power $\left.\left(\left(x(i, 5)-x_{-} t\right), 2\right)+\operatorname{power}\left(\left(y(i, 5)-y_{-} t\right), 2\right)\right)$;

dist_vect $(\mathrm{i},:)=[\mathrm{dr} 1 \mathrm{dr} 2 \mathrm{dr} 3 \mathrm{dr} 4 \mathrm{dr} 5]$;

$\operatorname{RSS} 1=\operatorname{Pr}(\mathrm{i}, 1)-$ cal_factor; 


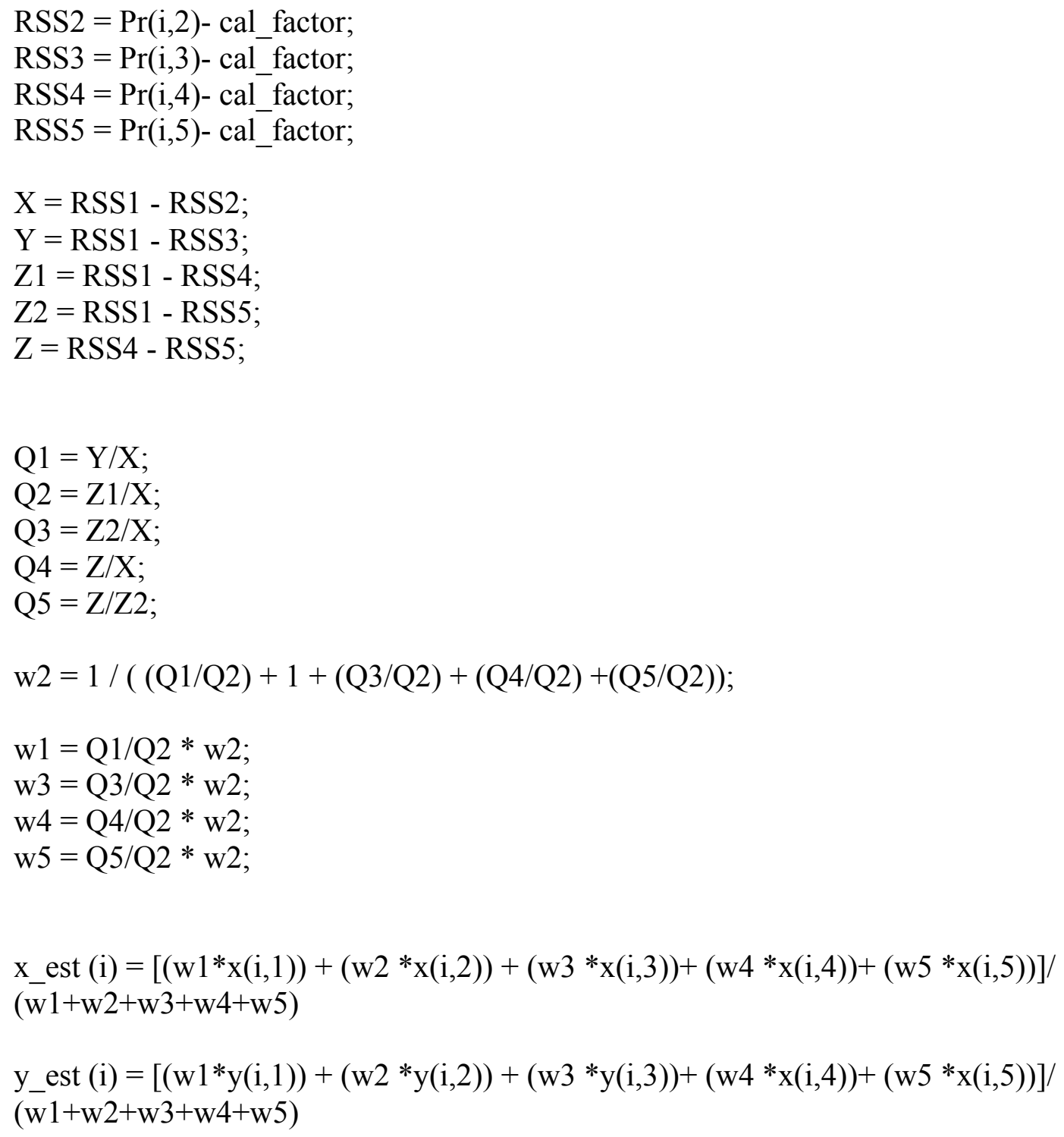

$\operatorname{RMSE}(i)=\operatorname{sqrt}\left(\operatorname{power}\left(\left(x_{-}\right.\right.\right.$est $\left.\left.(i)-x \_t\right), 2\right)+\operatorname{power}\left(\left(y \_\right.\right.$est $\left.\left.\left.(i)-y \_t\right), 2\right)\right)$;

final_matrix_cal_CDRSS $(i, 1)=$ cal_factor; final_matrix_cal_CDRSS $(\mathrm{i}, 2)=\mathrm{RM} \overline{\mathrm{MSE}}(\mathrm{i})$;

end

dist_vect

final_matrix_cal_CDRSS 


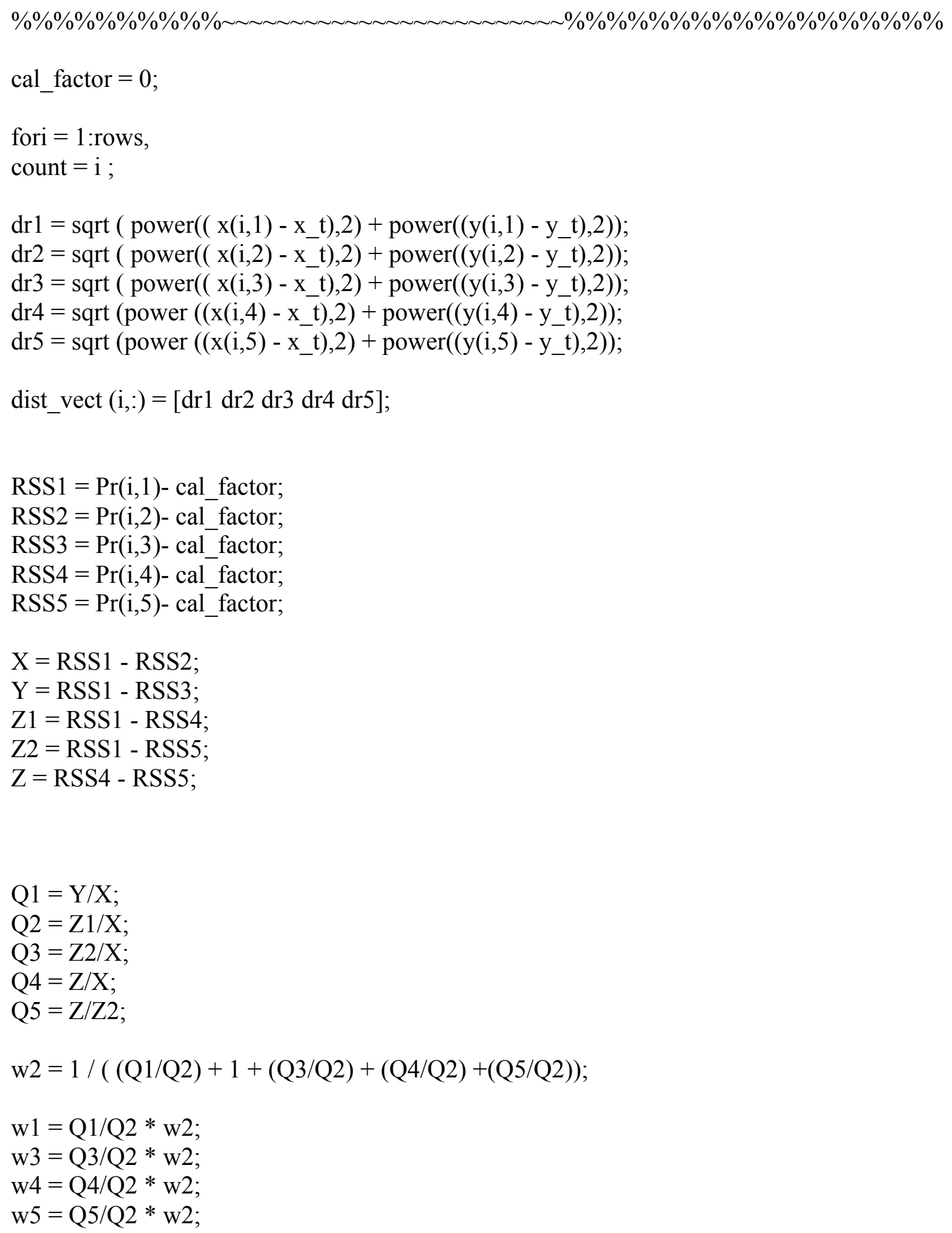


$\mathrm{x} \_$est $(\mathrm{i})=\left[\left(\mathrm{w} 1 *_{\mathrm{x}}(\mathrm{i}, 1)\right)+(\mathrm{w} 2 * \mathrm{x}(\mathrm{i}, 2))+\left(\mathrm{w} 3 *_{\mathrm{x}}(\mathrm{i}, 3)\right)+\left(\mathrm{w} 4 *_{\mathrm{x}}(\mathrm{i}, 4)\right)+\left(\mathrm{w} 5 *_{\mathrm{x}}(\mathrm{i}, 5)\right)\right] /$ $(\mathrm{w} 1+\mathrm{w} 2+\mathrm{w} 3+\mathrm{w} 4+\mathrm{w} 5)$

$\mathrm{y} \_$est $(\mathrm{i})=\left[\left(\mathrm{w} 1{ }^{*} \mathrm{y}(\mathrm{i}, 1)\right)+(\mathrm{w} 2 * \mathrm{y}(\mathrm{i}, 2))+(\mathrm{w} 3 * \mathrm{y}(\mathrm{i}, 3))+(\mathrm{w} 4 * \mathrm{x}(\mathrm{i}, 4))+(\mathrm{w} 5 * \mathrm{x}(\mathrm{i}, 5))\right] /$ $(\mathrm{w} 1+\mathrm{w} 2+\mathrm{w} 3+\mathrm{w} 4+\mathrm{w} 5)$

$\operatorname{RMSE}(i)=\operatorname{sqrt}\left(\operatorname{power}\left(\left(x_{-}\right.\right.\right.$est $\left.\left.(i)-x_{-} t\right), 2\right)+\operatorname{power}\left(\left(y \_\right.\right.$est $\left.\left.\left.(i)-y \_t\right), 2\right)\right)$;

final_matrix_cal_CDRSS $(i, 1)=$ cal_factor;

final_matrix_cal_CDRSS $(i, 2)=$ RMSE(i);

end

dist_vect

final_matrix_cal_CDRSS 


\section{APPENDIX B. Permission from Matt Ettus}

Permission from Matt Ettus to use the materials from the Ettusnad GNU Radio web site.

Gmail - Regd: Permission to use some materials from Ettus website and G... https: $/ /$ mail.google.com $/ \mathrm{mail} / \mathrm{u} / 0 /$ ?uil=2\&il=563cf35ac3 \&view=pt\&q=ma.

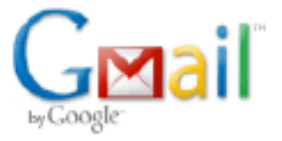

Gayathri Ramasubramanian <gayathri.ramasubramanian@gmail.com>

Regd: Permission to use some materials from Ettus website and Gnu Radio link 2 messages

Gayathri Ramasubramanian <gayathri.ramasubramanian@gmail.com> To: matt@ettus.com

Mon, Sep 1, 2014 at 4:07 PM

$\mathrm{Hi}$

I am working on a masters thesis based on performance characterization of USRP and some of its applications ( mainly wrt USRPN210 and USRP2 devices with WBX and SBX daughter boards)

I would like to use some of photos from the website (esp for the above mentioned devices).

Kindly grant me permission for the same and maybe a few others.

Also I am using some flow graphs (predefined ones like UHD_FFT.py, UHD_SIGGEN.py, some developed with help of GNU Radio experts). Please grant permission for them too.

Look forward to your response.

Thanks

Regards

Gayathri

Matt Ettus<matt@ettus.com>

Mon, Sep 1, 2014 at 4:53 PM

To: Gayathri Ramasubramanian <gayathri.ramasubramanian@gmail.com>

Yes, you may use all of that. Good luck with your thesis. I would love to have a copy when your are done.

Matt

[Quoted text hidden] 